\title{
Waldkonversion und Bodendegradation im tropischen Tiefland von Ostbolivien
}

GIS-gestützte Analyse zur Regionalisierung der Bodendegradation im Department Santa Cruz

\author{
Dissertation \\ zur Erlangung des Doktorgrades \\ der Mathematisch-Naturwissenschaftlichen Fakultäten \\ der Georg-August-Universität zu Göttingen
}

vorgelegt von
Jens-Peter Krüger

aus Hamburg

Göttingen 2005 
D 7

Referentin/Referent: Prof. Dr. Gerhard Gerold

Korreferentin/Korreferent: Prof. Dr. Bernd Cyffka

Tag der mündlichen Prüfung: 20.01.2006 




\section{GLIEDERUNG}

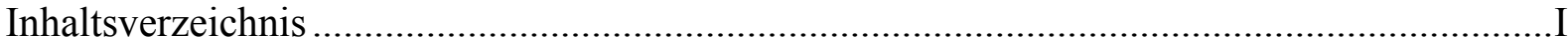

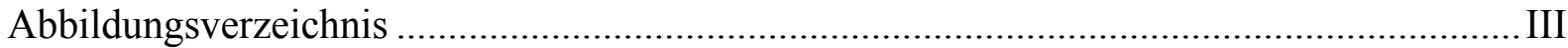

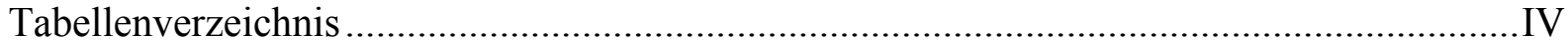

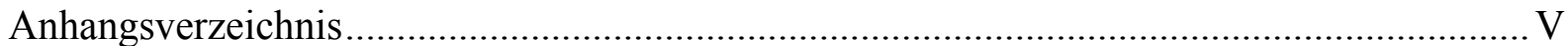

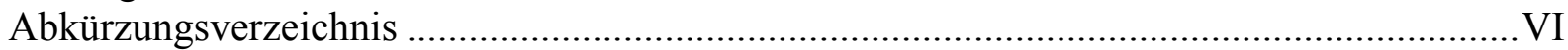

\section{Inhaltsverzeichnis}

1. Einleitung ................................................................................................................................... 1

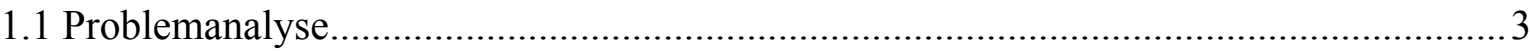

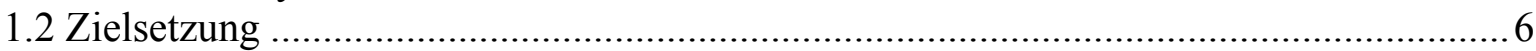

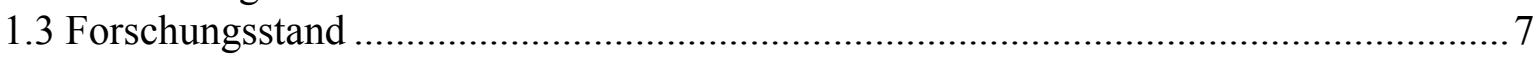

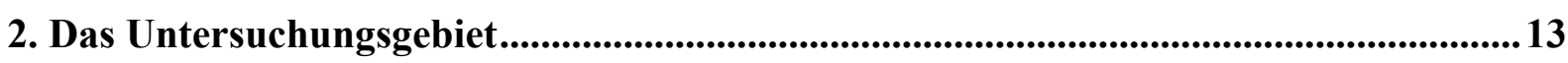

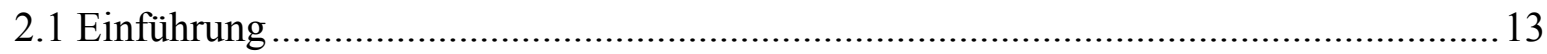

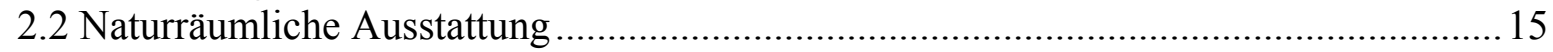

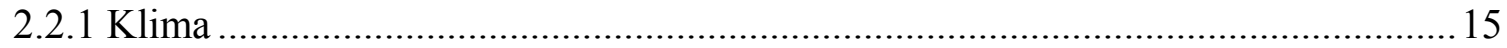

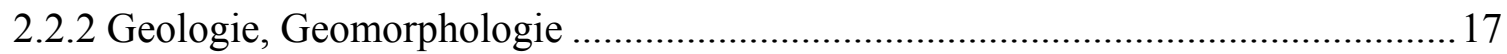

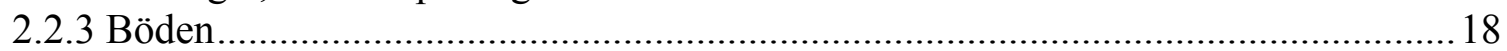

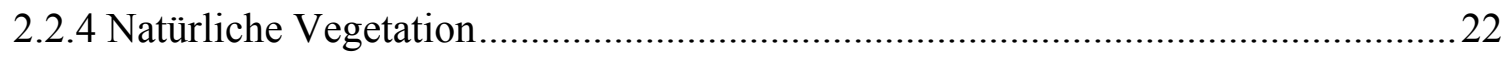

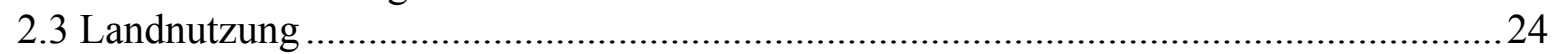

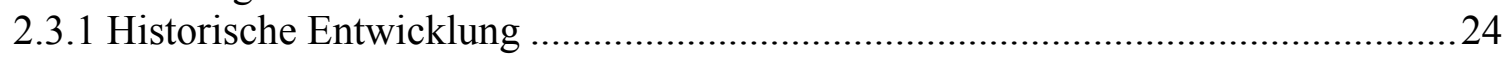

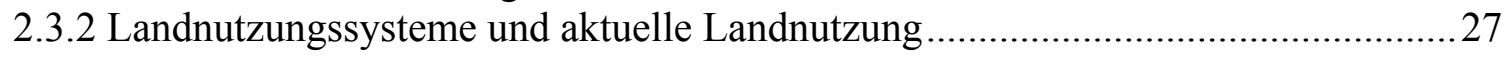

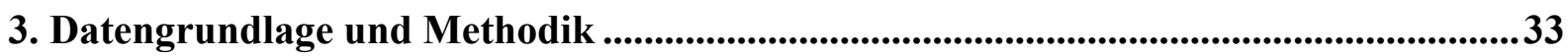

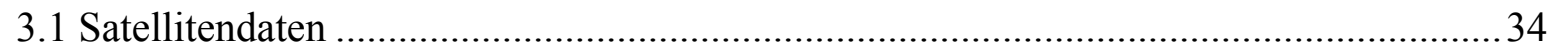

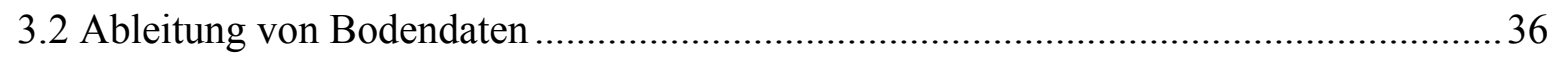

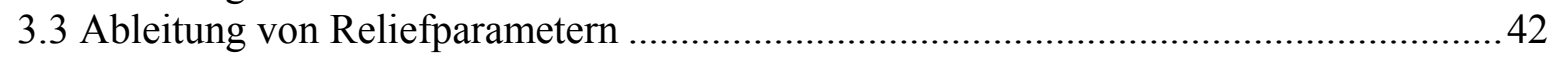

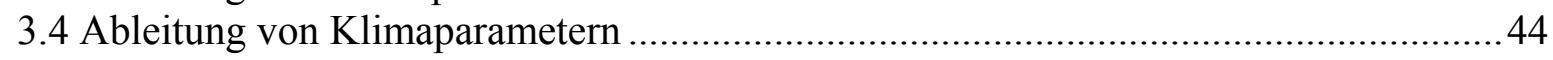

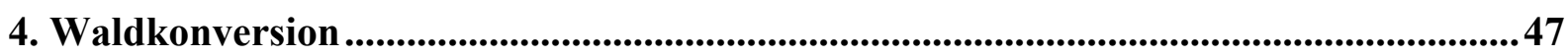

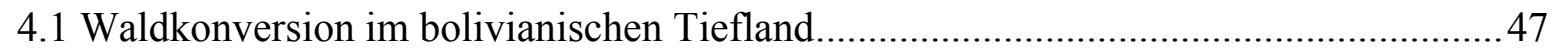

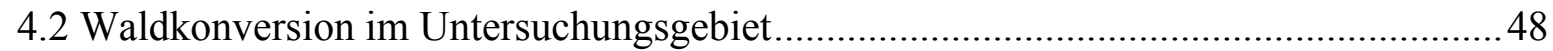

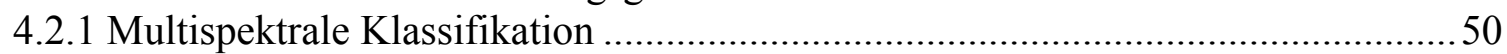

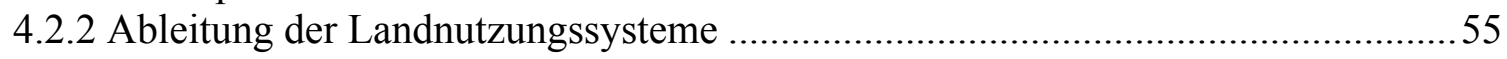

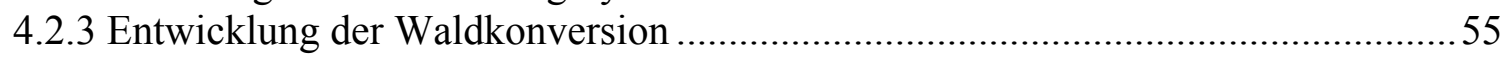

4.2.4 Anteil der Produzentengruppen an der Waldkonversion........................................63

4.2.5 Entwicklung der Landwirtschaftsflächen im Untersuchungsgebiet ........................66

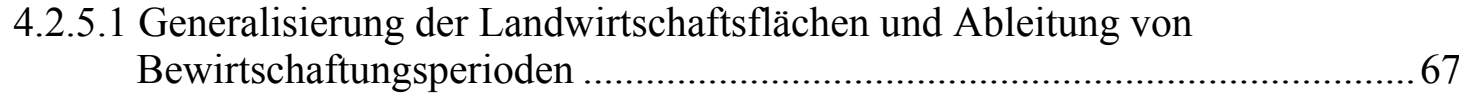

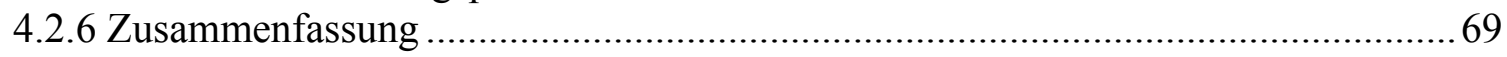

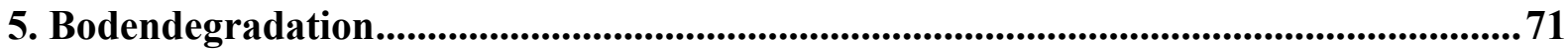

5.1 Bodendegradation im ostbolivianischen Tiefland ...................................................... 71

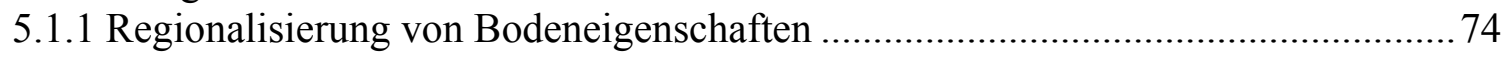

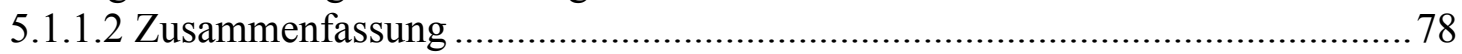


5.1.2 Änderung von SOC und $\mathrm{N}_{\mathrm{t}}$ unter ackerbaulicher Nutzung in den Tropen und Subtropen

5.1.2.1 Ableitung relativer SOC und $\mathrm{N}_{\mathrm{t}}$-Verluste - Literaturreview .......................... 81

5.1.2.2 Ableitung initialer SOC- und $\mathrm{N}_{\mathrm{t}}$-Gehalte im Untersuchungsgebiet................ 87

5.1.2.3 SOC- und $\mathrm{N}_{\mathrm{t}}$-Verluste im Untersuchungsgebiet - regionale Analyse.............. 89

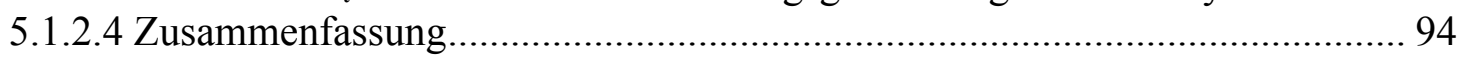

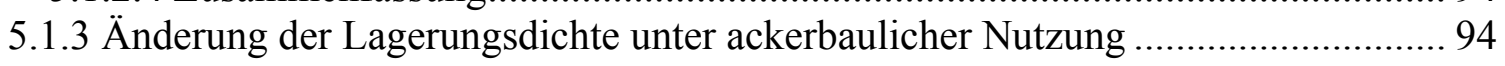

5.1.3.1 Ableitung initialer Lagerungsdichten im Untersuchungsgebiet...................... 99

5.1.3.2 Bodenverdichtung im Untersuchungsgebiet - regionale Analyse................. 100

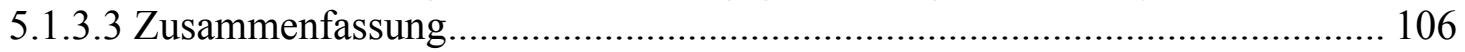

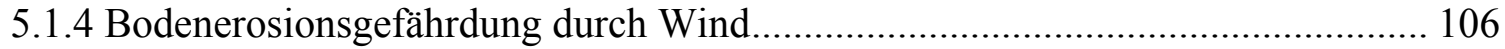

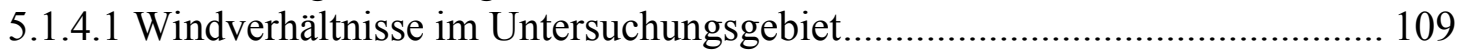

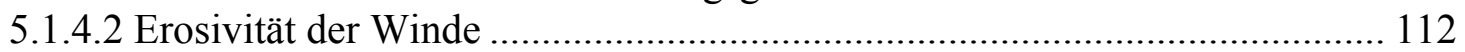

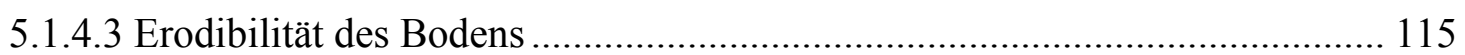

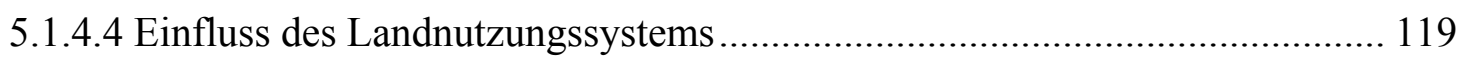

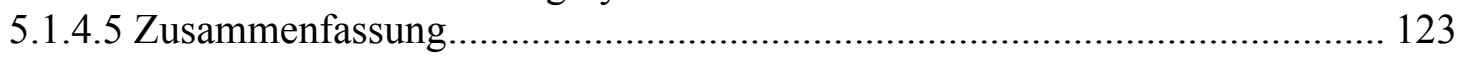

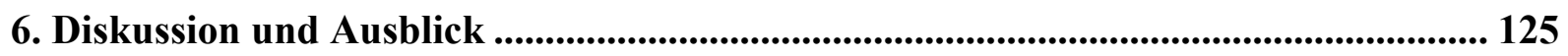

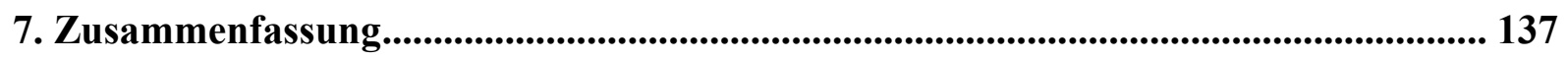

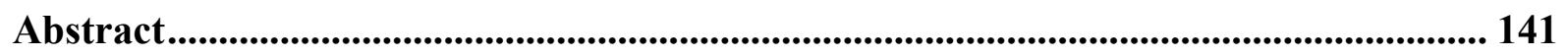

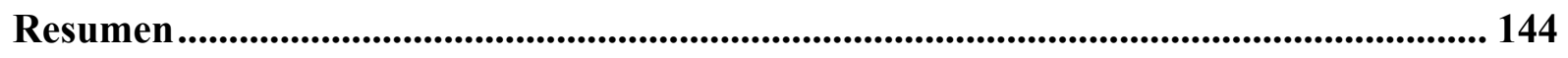

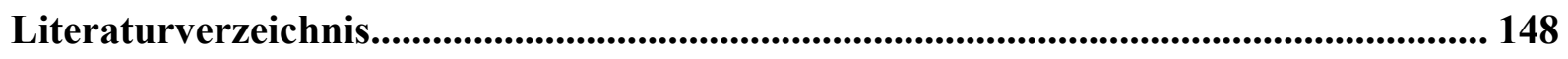

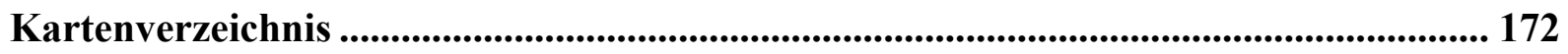

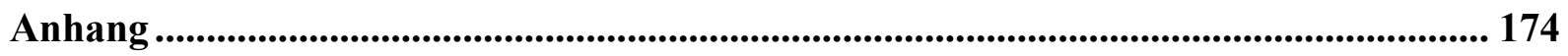




\section{Abbildungsverzeichnis}

Abb. 1: Potenzielle Auswirkungen landwirtschaftlicher Bodenbearbeitung ......................... 2

Abb. 2.1: Lage des Department Santa Cruz innerhalb Boliviens............................................. 13

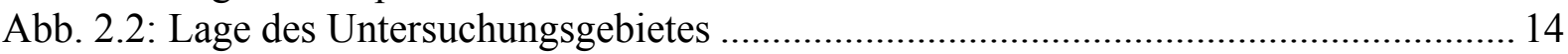

Abb. 3: Klimadiagramme der Stationen San Juan de Yapacani, Santa Cruz und Armando

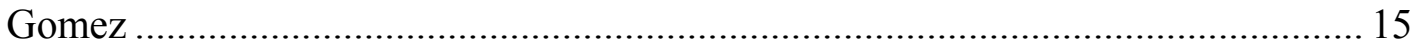

Abb. 4: Mittlere Korngrößenzusammensetzung der Böden im Untersuchungsgebiet.......... 19

Abb. 5: Verbreitung der natürlichen Vegetation im Department Santa Cruz ....................... 23

Abb. 6.1: Entwicklung des Sojabohnenanbaus im Department Santa Cruz von 1972 bis 2002

Abb. 6.2: Luftbildaufnahmen landwirtschaftlicher Betriebssysteme im Department Santa Cruz

Abb 7: Schema der Vorgehensweise.

Abb. 8: Räumliche Anordnung der Landsat Szenen (WRS Kombination) im Department

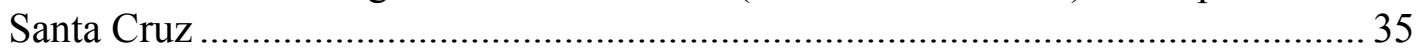

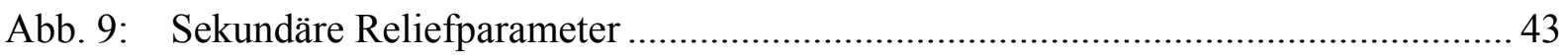

Abb. 10: Klimavariablen Niederschlag, Temperatur, Strahlung.......................................... 45

Abb. 11: Landnutzung und Vegetation 2001, basierend auf Klassifikation von Landsat 7-

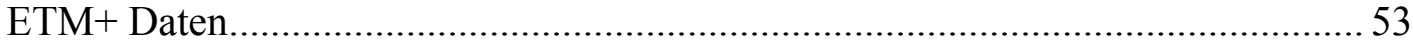

Abb. 12: Erscheinungsmuster der Landnutzungssysteme im Landsat-7 ETM+ Bild ............. 54

Abb. 13: Waldkonversion 1984-2001, basierend auf Klassifikation von Landsat 4, 5 TM und

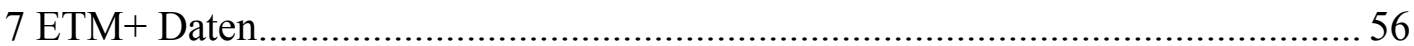

Abb. 14: Zunahme der Rodungsfläche im Untersuchungsgebiet 1984-2001 .........................57

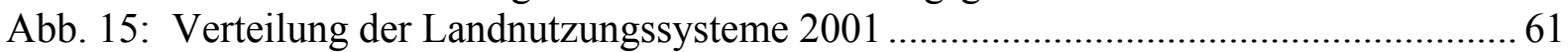

Abb. 16: Entwicklung der Produktionsfläche für Sommersoja in den 1990er Jahren nach

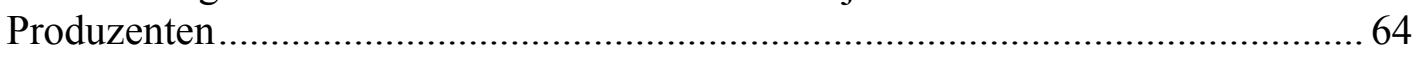

Abb. 17: Expansion landwirtschaftlicher Nutzflächen nach Produzenten 1984-2001............ 64

Abb. 18: Anteil an Sekundärwäldern in den Gebieten der verschiedenen Produzenten......... 65

Abb. 19: Streudiagramm der gemessenen und regionalisierten Sand- und Schluffgehalte im

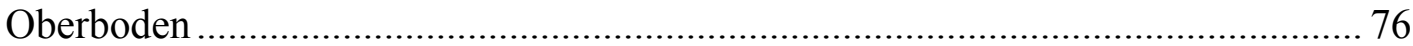

Abb. 20: regionalisierte Bodenarten und DGM des Untersuchungsgebiets .......................... 77

Abb. 21: relative SOC- und $\mathrm{N}_{\mathrm{t}}$-Verluste (Vorräte) in Abhängigkeit von der Nutzungsdauer (Oberboden)

Abb. 22: Boxplots der SOC- und $\mathrm{N}_{\mathrm{t}}$-Ausgangsgehalte nach Regionen ................................. 88

Abb. 23: Boxplots der SOC- und $\mathrm{N}_{\mathrm{t}}$-Ausgangsgehalte in Abhängigkeit vom Tongehalt....... 88

Abb. 24: Mittlere SOC- und $\mathrm{N}_{\mathrm{t}}$-Vorräte in den Oberböden von Ackerflächen 2001 ............. 93

Abb: 25: Boxplots der initialen Lagerungsdichten in Abhängigkeit von der Bodenart

(Oberboden)

Abb. 26: Lagerungsdichten, ab denen Einschränkungen im Wurzelwachstum auftreten.... 102

Abb. 27: Mittlere Lagerungsdichten im Oberboden von Ackerflächen 2001 ..................... 105

Abb. 28: Schwellenwerte der Windgeschwindigkeit zur Erosion von Bodenpartikeln in $15 \mathrm{~cm}$ Höhe

Abb. 29: Winderosionsereignisse im südlichen Untersuchungsgebiet (05.09.2002)........... 108

Abb. 30: Vorherrschende Windrichtungen im Untersuchungsgebiet .................................. 110

Abb. 31: Monatliche Tagesmaxima der Windgeschwindigkeit 1980 ................................ 111

Abb. 32: Windprofile bei unterschiedlicher Bodenrauhigkeit ........................................... 112

Abb. 33: Berechnete Häufigkeitsverteilung der Windgeschwindigkeit in \% nach Rayleigh 114 
Abb. 34: Kurvenfit nach dem Algorithmus von FREDLUND et al. (2000) für sandige Lehme

Abb. 35: Einfluss von Textur, SOM-Gehalt und Aggregierung sandiger Lehme auf die Erodibilität

Abb. 36: Einfluss von Textur, SOM-Gehalt und Aggregierung sandiger Lehme auf die Erodibilität.

Abb. 37: Potenzielles Winderosionsrisiko auf Grundlage der Bodenart und der Topographie

Abb. 38: Windschutzstreifen in der Mennonitenkolonie Riva Palacios

Abb. 39: Beziehung zwischen jährlichem Niederschlag (Station El Trompillo, Santa Cruz) und Southern Oscillation Index (SOI).

Abb. 40: Häufigkeiten extrem trockener bzw. extrem feuchter Jahre (Station El Trompillo, Santa Cruz).

Abb. 41: Änderung des SOC-Vorrats nach Umstellung von konventioneller auf reduzierte Bodenbearbeitung $(0-20 \mathrm{~cm})$.

\section{Tabellenverzeichnis}

Tab. 1: Statistische Parameter verschiedener Klimastationen im Department Santa Cruz.. 16

Tab. 2: Regionale Einordnung bodenchemischer Parameter in Ostbolivien....................... 20

Tab. 3: Anbauflächenentwicklung (in ha) der wichtigsten landwirtschaftlichen Produkte im

Department Santa Cruz (1985-2002) ............................................................... 26

Tab. 4: Verteilung der Landnutzung im Department Santa Cruz....................................... 31

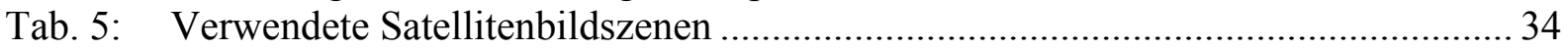

Tab. 6: Verwendete Profilbeschreibungen der Bodendatenbank ....................................... 37

Tab. 7: Korngrößenklassen nach USDA/FAO und AG Boden ........................................ 38

Tab. 8: Korrelationsmatrix Oberbodenparameter unter natürlicher Vegetation.................... 39

Tab. 9: Korrelationsmatrix Oberbodenparameter unter landwirtschaftlicher Nutzung........ 39

Tab. 10: Waldkonversion in Bolivien............................................................................... 49

Tab. 11: Flächenanteil der Landbedeckungsklassen (Untersuchungsgebiet) .........................5 58

Tab. 12: Veränderung des Flächenanteils der Landbedeckungsklassen (Untersuchungsgebiet)

Tab. 13: Entwaldungsrate (Untersuchungsgebiet)

Tab. 14: Flächenanteil der Landbedeckungsklassen (nördliches Untersuchungsgebiet, WRS$2230 / 72)$

Tab. 15: Veränderung des Flächenanteils der Landbedeckungsklassen (nördliches

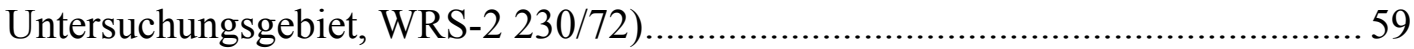

Tab. 16: Entwaldungsrate (nördliches Untersuchungsgebiet, WRS-2 230-72).....................59

Tab. 17: Flächenanteil der Landbedeckungsklassen (südliches Untersuchungsgebiet, WRS-2 230/73)

Tab. 18: Veränderung des Flächenanteils der Landbedeckungsklassen (südliches Untersuchungsgebiet, WRS-2 230/73)

Tab. 19: Entwaldungsrate (südliches Untersuchungsgebiet, WRS-2 230-73) .......................60

Tab. 20: Änderung der Klassenanteile an der Landnutzungsfläche 1984-2001 in [\%].......... 61

Tab. 21: Flächenanteile der Produzentengruppen an der Landnutzungsfläche 1984-2001 .... 62

Tab. 22: Flächenanteile der Produzentengruppen an der Rodung 1984-2001 
Tab. 23: Hauptsächliche Entwicklungen landwirtschaftlicher Nutzflächen im Zeitraum 19842001

Tab. 24: Einteilung der Bewirtschaftungsperioden

Tab. 25: Korrelationskoeffizienten [r] von Klima- und Reliefparametern mit Oberbodenvariablen unter natürlicher Vegetation

Tab. 26: SOC- und $\mathrm{N}_{\mathrm{t}}$-Verluste im Oberboden nach Konversion natürlicher Vegetation in eine ackerbauliche Nutzung im tropischen Tiefland von Ostbolivien....

Tab. 27: Mittlere prozentuale Rückgänge der SOC- und $\mathrm{N}_{\mathrm{t}}$-Gehalte unter moderner und traditioneller Bewirtschaftung nach mehr als 10 Jahren (10-70 a) kontinuierlicher Nutzung (Oberboden)

Tab. 28: Korrelationsmatrix von Gesamtverlusten und jährlicher Raten für SOC und $\mathrm{N}_{\mathrm{t}}$ aller analysierten Studien (Oberboden)

Tab. 29: Relative SOC- und $\mathrm{N}_{\mathrm{t}}$-Verluste in Abhängigkeit von Nutzungsdauer und Tongehalt (Oberboden)

Tab. 30: Mittlerer SOC-, SOM- und $\mathrm{N}_{\mathrm{t}}$-Status im Oberboden unter natürlicher Vegetation in Abhängigkeit vom Tongehalt.

Tab. 31: Mittlere SOC-Verluste im Oberboden in Abhängigkeit von Nutzungsdauer und Tongehalt

Tab. 32: Mittlere $\mathrm{N}_{\mathrm{t}}$-Verluste im Oberboden Abhängigkeit von Nutzungsdauer und Tongehalt.

Tab. 33: Mittlere Gehalte und Vorräte organischer Substanz (SOM) der Anbauflächen 2001

Tab. 35: Veränderung von Lagerungsdichte und Porenvolumen unter kontinuierlichem

Ackerbau im Untersuchungsraum.

Tab. 34: Einordnung der Bodenvorräte organischer Substanz 92 .98

Tab. 36: Allgemeine Beziehung zwischen Lagerungsdichte und Wurzelwachstum auf Grundlage der Bodenart ....

Tab. 37: Ausgangsniveau und mittlere Veränderung von Lagerungsdichte und Porenvolumen durch mechanische Bodenbearbeitung 101

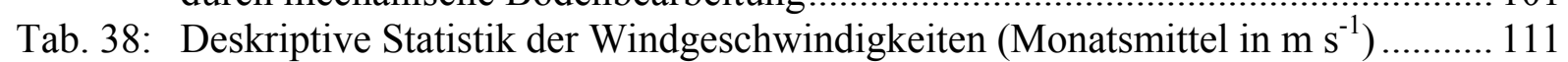

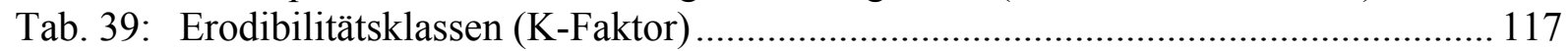

Tab. 40: Korrelationskoeffizienten des K-Faktors mit SOM-Gehalt, Aggregierung und Textur

Tab. 41: Jährliche Abholzungsraten in ausgewählten tropischen Regionen mit hoher Waldkonversion 126

\section{Anhangsverzeichnis}

Tab. A1: Richtwerte chemischer Bodeneigenschaften nach CIAT.... 174

Tab. A2: Technische Daten der verwendeten Landsat Satellitenbilder ............................... 174

Tab. A3: Fehlermatrizen (confusion matrix) der Satellitenbildanalyse (Maximum-Likelihood,

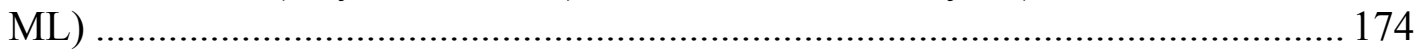

Tab. A4: Anbaukalender der Hauptanbaufrüchte im Department Santa Cruz....................... 176

Tab. A5: Allgemeine Beschreibung der Standorte und relative Abnahme von Bodenkohlenstoff (SOC) und Bodenstickstoff $\left(\mathrm{N}_{\mathrm{t}}\right)$ nach Konversion natürlicher Vegetation in landwirtschaftliche Kulturflächen der durchgesehenen Studien (Literaturreview) 


\section{Abkürzungsverzeichnis}

Abkürzungen von Programmen und Institutionen

ASSOD

Assessment of the Status of Human-Induced Soil

Degradation

CIAT Centro de Investigacíon Agrícola Tropical

FAN Fundación Amigos de la Naturaleza

GLASOD Global Assessment of Soil Degradation

MBAT Misión Británica en Agricultura Tropical

NRCS Natural Resources Conservation Service

PLUS Plan de Uso de Suelo

SENAMHI Servicio Nacional de Meteorología e Hidrología (staatlicher Wetterdienst)

SOVEUR Soil Vulnerability Assessment in Central and Eastern Europe

SRTM Shuttle Radar Topography Mission

bodenchemische und -physikalische Symbole, chemische Verbindungen

$\begin{array}{ll}\mathrm{Al} & \text { Aluminium } \\ \mathrm{BS} & \text { Basensättigung } \\ \mathrm{C} & \text { Kohlenstoff } \\ \mathrm{CO}_{2} & \text { Kohlendioxid } \\ \mathrm{d}_{\mathrm{b}} & \text { Lagerungsdichte } \\ \mathrm{Fe} & \text { Eisen } \\ \mathrm{ffS} & \text { Feinstsand } \\ \mathrm{fS} & \text { Feinsand } \\ \mathrm{fU} & \text { Feinschluff } \\ \mathrm{gS} & \text { Grobsand } \\ \mathrm{gU} & \text { Grobschluff } \\ \mathrm{K} & \text { Kalium } \\ \mathrm{KAK} & \text { Kationenaustauschkapazität } \\ \mathrm{mS} & \text { Mittelsand } \\ \mathrm{N} & \text { Stickstoff }\end{array}$

\begin{tabular}{|c|c|}
\hline $\mathrm{N}_{\mathrm{t}}$ & Gesamtstickstoff \\
\hline $\mathrm{P}$ & Phosphor \\
\hline $\mathrm{pH}$ & $\begin{array}{l}\text { negativer dekadischer } \\
\text { Logarithmus der } \mathrm{H}_{3} \mathrm{O}^{+} \\
\text {Ionenkonzentration }\end{array}$ \\
\hline SOC & Soil Organic Carbon \\
\hline SOM & Soil Organic Matter \\
\hline $\mathrm{T}$ & Ton \\
\hline $\mathrm{U}$ & Schluff \\
\hline
\end{tabular}

Statistische Parameter

$\begin{array}{ll}\text { Max } & \text { Maximum } \\ \text { Min } & \text { Minimum } \\ \text { MW } & \text { arithmetischer Mittelwert } \\ n & \text { Anzahl (Stichprobe) } \\ \mathrm{r} & \text { Korrelationskoeffizient } \\ \mathrm{r}^{2} & \text { Bestimmtheitsmaß } \\ \text { Stabw } & \text { Standardabweichung }\end{array}$

\section{Reliefparameter}

$\begin{array}{ll}\text { AD } & \begin{array}{l}\text { Altitude above drain } \\ \text { culmination (Höhe über } \\ \text { Tiefenlinie) } \\ \text { Altitude below summit } \\ \text { culmination (Höhe unter } \\ \text { Scheitellinie) }\end{array} \\ \text { NA } & \begin{array}{l}\text { Normalized altitude } \\ \text { (normierte Höhe) }\end{array} \\ & \text { SAGA-Wetness Index } \\ \text { WI }_{\mathrm{S}} & \text { (SAGA-Bodenfeuchte- } \\ & \text { Index) } \\ & \\ \text { sonstige Abkürzungen und Symbole } \\ \sim\end{array}$




\begin{tabular}{|c|c|c|c|}
\hline$\alpha$ & Signifikanzniveau & $\mathrm{m}$ ü. NN & Meter über Normalnull \\
\hline$\pi$ & Kreiszahl (Pi 3,14159) & mündl. Mitt. & mündliche Mitteilung \\
\hline Abb. & Abbildung & $\mathrm{N}$ & Nord \\
\hline Anm. & Anmerkung & $\mathrm{n}$. & nach \\
\hline BIP & Bruttoinlandsprodukt & n.s. & nicht signifikant \\
\hline boliv. & bolivianischer & NDVI & Normalized Difference \\
\hline bspw. & beispielsweise & & Vegetation Index \\
\hline bzw. & beziehungsweise & NE & Nordosten \\
\hline ca. & cirka & NW & Nordwesten \\
\hline $\mathrm{cm}$ & Zentimeter & o. Bez. & ohne Bezeichnung \\
\hline $\mathrm{cmol}$ & Zentimol & ppm & parts per million \\
\hline Dep. & Department & PV & Porenvolumen \\
\hline DGM & Digitales Geländemodell & $\mathrm{S}$ & Süd \\
\hline $\mathrm{E}$ & Ost & $\mathrm{s}$ & Sekunde \\
\hline Ed. & Editor & $\mathrm{SE}$ & Südosten \\
\hline et al. & und andere & südl. & südlich \\
\hline etc. & et cetera & SW & Südwesten \\
\hline $\exp$ & Exponent & Tab. & Tabelle \\
\hline GCM & General Circulation Models & $\mathrm{TM}$ & Thematic Mapper \\
\hline Gew.- \% & Gewichtsprozent & ü. NN & über Normal Null \\
\hline GIS & Geographische & u.a. & unter anderem \\
\hline & Informationssysteme & v.a. & vor allem \\
\hline GPS & Global Positioning System & W & West \\
\hline h & Stunde & WRS & World Reference System \\
\hline ha & Hektar & z.T. & zum Teil \\
\hline i.V. & in Vorbereitung & & \\
\hline ISODATA & Iterative Self-Organizing & \multirow{2}{*}{\multicolumn{2}{|c|}{$\begin{array}{l}\text { Bodenarten nach SOIL SURVEY STAFF } \\
(2003)\end{array}$}} \\
\hline \multirow{2}{*}{ ITCZ } & $\begin{array}{l}\text { Data Analysis Technique } \\
\text { Innertronische }\end{array}$ & & \\
\hline & Konvergenzzone & $\mathrm{C}$ & clay (Ton) \\
\hline JM & Jeffries-Matusita & $\mathrm{CL}$ & clay loam (toniger Lehm) \\
\hline Kap. & Kapitel & $\mathrm{L}$ & loam (Lehm) \\
\hline $\mathrm{kg}$ & Kilogramm & $\mathrm{LS}$ & loamy sand (lehmiger Sand) \\
\hline $\mathrm{km}$ & Kilometer & $\mathrm{SCL}$ & sandy clay loam (sandig \\
\hline $\mathrm{km}^{2}$ & Quadratkilometer & & toniger Lehm) \\
\hline $\begin{array}{l}\text { Landsat-TM } \\
\text { lat }\end{array}$ & $\begin{array}{l}\text { Landsat Thematic Mapper } \\
\text { latitude }\end{array}$ & SICL & silty clay loam (schluffig \\
\hline $\ln$ & Logarithmus & SIL & silty loam (schluffiger \\
\hline long & longitude & & Lehm) \\
\hline $\mathrm{m}$ & Meter & SL & sandy loam (sandiger \\
\hline $\mathrm{mg}$ & Milligramm & & Lehm) \\
\hline ML & Maximum-Likelihood & & \\
\hline $\mathrm{mm}$ & Millimeter & & \\
\hline
\end{tabular}




\section{Danksagung}

Die vorliegende Arbeit wurde an der Abteilung Landschaftsökologie des Geographischen Instituts der Universität Göttingen in einem von der Deutschen Forschungsgemeinschaft (DFG) gefördertem Projekt zur Analyse der Waldkonversion und Bodendegradation in Ostbolivien angefertigt. Dieses wurde in enger Kooperation mit der Fundación Amigos de la Naturaleza (FAN) in Santa Cruz, Bolivien durchgeführt. Zahlreiche Personen haben zum Gelingen dieser Arbeit beigetragen, denen ich an dieser Stelle dafür meinen herzlichen Dank aussprechen möchte.

Ein großer Dank geht an Prof. Dr. Gerhard Gerold für die gemeinsame Ausarbeitung und erfolgreiche Umsetzung des Projektes sowie, damit verbunden, für die Betreuung der Dissertation. Herzlichen Dank auch an Prof. Dr. Bernd Cyffka, der sich freundlicherweise bereit erklärte das Korreferat zu übernehmen.

Für die überaus herzliche Aufnahme und große Hilfsbereitschaft möchte ich allen Mitarbeiterinnen und Mitarbeitern von FAN danken. Stellvertretend für das gesamte Team seien Natalia Araujo und Prof. Dr. Pierre Ibisch genannt. Vielen Dank an Christoph Nowicki, der mir zahlreiche Daten überlassen hat und wertvolle Tipps gab. Ebenso hilfreich waren die Anregungen von Jorge Choquehuanca und Dr. Rolf Wachholtz. Ihnen sei herzlichst gedankt. Bei Carola Sierra und Stefan Kreft bedanke ich mich zudem für ihre große Hilfe beim Auffinden grauer Literatur. Vielen Dank auch an Arnélida Gorrin für ihre vielen tollen Hilfeleistungen.

Ein besonderer Dank gilt den Kolleginnen und Kollegen des Geographischen Instituts, die diese Arbeit in vielerlei Hinsicht konstruktiv unterstützt haben. Ganz besonders erwähnt sei an dieser Stelle PD Dr. Jürgen Böhner, ohne dessen Anregungen und Hilfestellungen diese Arbeit nicht in der vorliegenden Form umgesetzt worden wäre. Für seine aktive und stets engagierte Mitarbeit im Projekt danke ich Alexander Kemp. Ganz herzlichen Dank an JP Dr. Beate Michalzik, Tanja Lange und Dr. Stefan Erasmi, die die Arbeit kritisch begutachtet und korrigiert haben. Bei Dorothea Röckl und Ingrid Kohlrautz-Weinrich bedanke ich mich sehr für ihre Mithilfe bei der Verwaltung des Projektes. Freundschaftliche Unterstützung, wertvolle Anstöße oder Hilfeleistungen durfte ich von Angela Kreikemeier, Dr. Elke Fischer, Claudia Sültmann, Annegret Kunzke, PD Dr. Heiko Faust, PD Dr. Stephan Glatzel, René Trujillo und Johannes Winter erfahren. Ihnen allen sei dafür ganz herzlich gedankt.

Den nötigen Rückhalt diese Arbeit zu einem erfolgreichen Abschluss zu führen, gab mir meine Familie. Maira, Lili und Uda, tausend Dank für Euer Durchhaltevermögen! Meinen Eltern, Ingrid und Erhard Krüger, danke ich, dass sie meinen Werdegang stets vertrauensvoll begleitet haben. 


\section{EINLEITUNG}

„Der Pflug kann heute ohne Zweifel als das wichtigste Instrument angesehen werden, das zur Zerstörung von Millionen Hektar fruchtbaren tropischen und subtropischen Bodens beigetragen hat. Es ist an der Zeit, dass wir ihn von dem hohem Sockel herunterholen, auf dem er sich in unserem Kulturverständnis befindet. “ (aus: DERPSCH 2000).

Diese Stellungnahme von Derpsch zeigt recht gut den Problemkreis auf, mit dem sich viele tropische und subtropische Länder heute auseinandersetzen müssen, seit vor einigen Jahrzehnten mechanisierte Bodenbearbeitungssysteme eingeführt wurden - Abnahme der Bodenproduktivität und Verlust landwirtschaftlicher Nutzfläche durch Bodendegradation sind die Folgen. Jährlich gehen mehrere Millionen Hektar Anbaufläche verloren und der Flächenanteil sinkender Produktivität liegt noch weitaus höher. Natürlich ist Bodendegradation ein weltweites Phänomen, dennoch sind die Böden der Tropen und Subtropen aufgrund ihrer Beschaffenheit und der klimatischen Verhältnisse gegenüber denen der gemäßigten Breiten weniger widerstandsfähig (WBGU 1994).

Bodenbearbeitungstechniken, die nicht an die speziellen Bedingungen in diesen Breiten angepasst sind oder unsachgemäß angewendet werden, bilden eine wesentliche Ursache der fortschreitenden Degradation tropischer und subtropischer Böden. Intensive Bodenbearbeitung führt in Kombination mit warm-feuchten Bedingungen $\mathrm{zu}$ einer schnellen Mineralisierung der organischen Substanz, mit Abbauraten, die deutlich über denen der Mittelbreiten liegen (BAYER et al. 2001, SIX et al. 2002). In Bezug auf den Verlust organischer Substanz durch landwirtschaftliche Aktivitäten sieht KATYAL (2001) insbesondere die ariden, semiariden und sub-humiden Tropen als äußerst gefährdet an. Abnehmende Humusgehalte bedeuten gleichzeitig eine Verringerung der Wasserspeicherkapazität und Nährstoffsorption sowie eine Verschlechterung der Bodenstruktur (BRONICK \& LAL 2005). Kontinuierlicher Anbau entzieht dem Boden pflanzenverfügbare Nährstoffe, die dieser aus ökonomischen Erwägungen heraus nicht in dem gleichen Maße zurück erhält. Anorganische Dünger sind teuer und eine wachsende Nachfrage infolge des schnellen Bevölkerungswachstum der letzten Jahrzehnte zwingt oft zu verkürzten Bracheperioden; dem Boden bleibt keine Zeit zur Regeneration. Die Zerstörung von Bodenaggregaten durch Pflugarbeiten und eine mangelnde Bodenbedeckung während der Bearbeitung und nach der Einsaat machen den Boden anfällig gegenüber Wind- und Wassererosion (LAL 1990, 2001); ein Effekt, der durch die hohe Intensität der Niederschläge und durch ausgeprägte Trockenzeiten in diesen Breiten verstärkt wird. Nährstoffreiche Feinbestandteile werden ausgeblasen oder verspült. Gleichzeitig führt der Einsatz von schwerem Gerät in der mechanisierten Landwirtschaft zur Ausbildung von Verdichtungen sowohl im Bereich der Pflugsohle als auch in größeren Bodentiefen (KOZLOWSKI 1999). Letzteres zeigt sich speziell bei Böden mit einem hohen Schluff- und Feinsandanteil, wenn diese zu einer Zeit befahren werden, in der sie aufgrund hoher Wassergehalte nur eine geringe Gefügestabilität besitzen. 
Obwohl in den ersten Jahren nach Rodung deutliche Ertragssteigerungen durch mechanisierte Bearbeitungstechniken zu verzeichnen sind, nimmt die Bodenproduktivität durch die Kombination der geschilderten Prozesse bereits nach wenigen Jahren ab. In der Regel sind tropische Böden, ohne Anwendung von konservierenden Maßnahmen, nach zwei bis drei Dekaden erschöpft (VLEK et al. 1997).

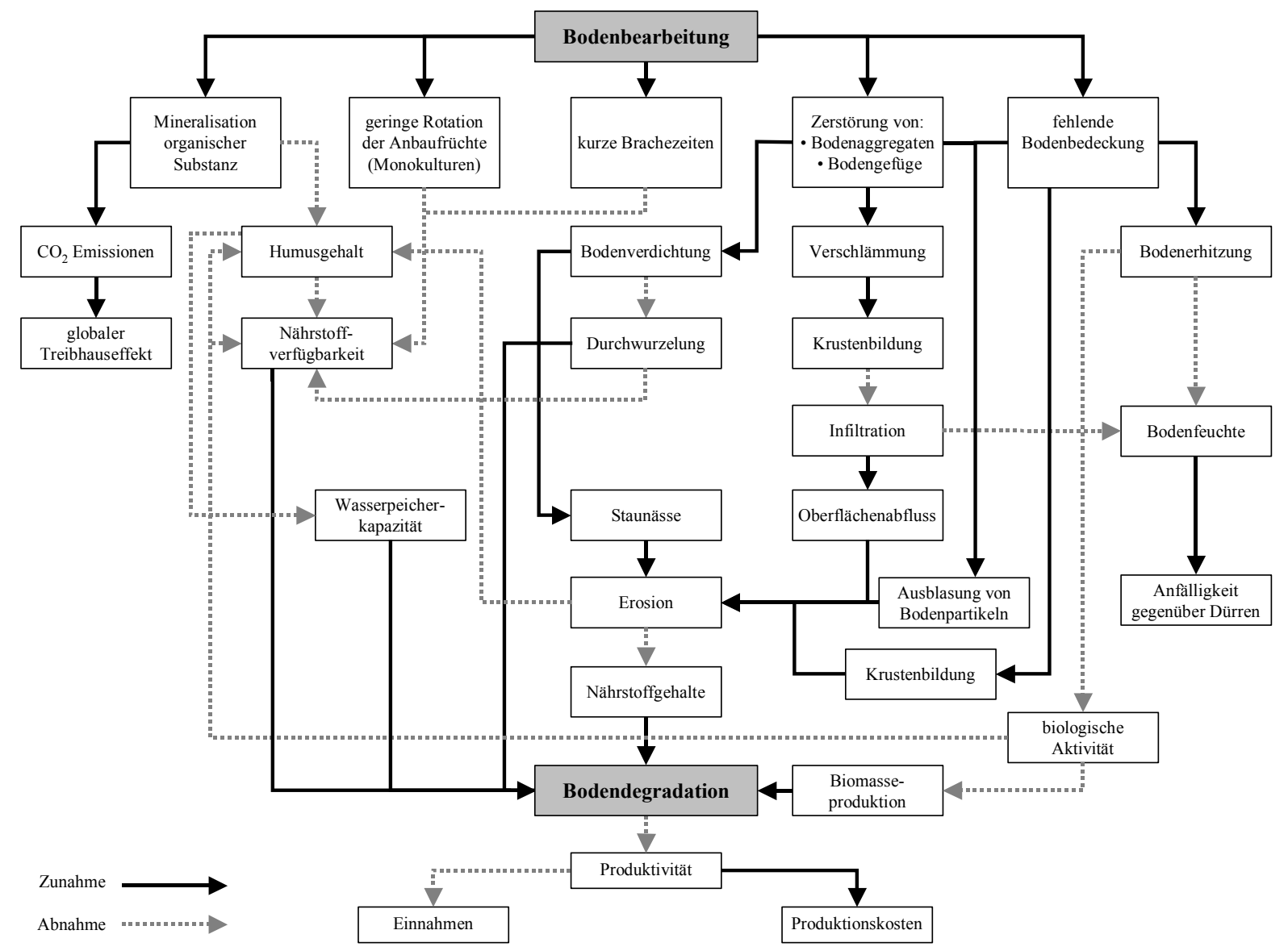

eigener Entwurf in Anlehnung an DERPSCH (2000)

Abb. 1: Potenzielle Auswirkungen landwirtschaftlicher Bodenbearbeitung

$\mathrm{Zu}$ den regionalen Ausprägungen fortschreitender Bodendegradation und deren Auswirkung auf die Nahrungsmittelproduktion gesellt sich noch eine globale Komponente: Da landwirtschaftlich genutzte Böden nur eine geringe Biomasseproduktion aufweisen, wird weniger $\mathrm{CO}_{2}$ im Boden gebunden, als durch den Abbau organischer Substanz freigesetzt wird (SCHLESINGER \& ANDREWS 2000). Der Boden verliert zunehmend seine Funktion als Kohlenstoffspeicher (LAL \& LOGAN 1995, SCHIMEL 1995). Potenzielle Folgen landwirtschaftlicher Bodenbearbeitung werden in Abbildung 1 angedeutet, wobei angesichts der komplexen Vorgänge im Boden naturgemäß nicht alle Zusammenhänge detailliert wiedergegeben werden. 
Bodendegradation ist ein schleichender Prozess, der, weil visuell kaum wahrnehmbar, normalerweise nicht als Problem angesehen wird. Erst massive Ertragseinbußen deuten das Ausmaß von Veränderungen im Boden zu einem Zeitpunkt an, an dem der Boden oft bereits irreversibel geschädigt ist. Sinkenden Erträgen wird häufig über die Rodung neuer Flächen, nicht selten in ökologisch sensiblen Gebieten oder Gebieten deren Böden nur bedingt für einen Anbau geeignet sind, begegnet. Investitionen in bodenkonservierende Anbautechniken werden häufig deswegen nicht vorgenommen, da sinkenden Arbeitseinkommen bei einem gleichzeitig höherem Arbeitsaufwand in den ersten Jahren eine gewisse Unsicherheit gegenübersteht, ob, und wenn ja in welchem Ausmaß, Produktionssteigerungen überhaupt zu erwarten sind (BARBIER 1997).

Um aus diesem Kreislauf auszubrechen, fordert DERPSCH (2000) in seiner eingangs zitierten Stellungnahme, dass für eine langfristige Sicherung der Bodenproduktivität ein Umdenken weg von bodenzehrenden hin $\mathrm{zu}$ bodenschonenden Landnutzungssystemen einsetzten muss. Sicherlich eine Forderung, die in einer an kurzfristigen Profiten orientierten und unter globalen Zwängen stehenden Gesellschaft nicht einfach umzusetzen ist. Gerade deswegen sind Studien, die sich mit dem Verlust der natürlichen Bodenproduktivität als Folge landwirtschaftlicher Nutzung befassen, von immenser Wichtigkeit.

\subsection{Problemanalyse}

Der Raum Santa Cruz, gelegen im gleichnamigen Department im tropischen Tiefland Ostboliviens, hat sich in den letzten 50 Jahren von einer unbedeutenden und zugleich abgeschiedenen Region im Zentrum Südamerikas zum wichtigsten Wirtschafts- und Handelszentrum Boliviens entwickelt. Die rasante Veränderung in diesem Zeitraum ist im wesentlichen auf einen wirtschaftlichen Boom der Sektoren Erdöl/Erdgas ${ }^{1}$ und Landwirtschaft zurückzuführen, in dessen Folge zahlreiche Maßnahmen zur infrastrukturellen Erschließung des ostbolivianischen Tieflandes umgesetzt wurden (Kap.2.3.1). Nach dem Niedergang des Bergbaus verband sich damit die Hoffnung, mittels einer besseren wirtschaftlichen Integration Boliviens, eine langfristige Konsolidierung der neuen Devisenquellen zu erzielen. Parallel sollte mit dem Ausbau des Agrarsektors die Versorgung des nationalen Marktes mit Nahrungsmitteln sichergestellt werden. Mit der wirtschaftlichen Erschließung des Departments Santa Cruz seit Anfang der 1950er Jahre hat sich die absolute Bevölkerungszahl bis heute auf über 2 Mio. Einwohner mehr als verachtfacht. Das mittlere Bevölkerungs-wachstum lag zwischen 1992 und 2001 bei 4,31 \% pro Jahr und damit 1,5\% über dem Landesdurchschnitt. Die Region Santa Cruz hat sich mit einem Anteil von über 30 $\%$ am Bruttoinlandsprodukt zur wirtschaftlich leistungsstärksten in Bolivien entwickelt, wovon wiederum der Hauptanteil auf den Agrarsektor entfällt; Schätzungen zufolge betrug

\footnotetext{
${ }^{1}$ Bolivien besitzt nach Venezuela die zweitgrößten Erdgasvorkommen und die größten ,freien’ Reserven in Südamerika
} 
der Anteil am BIP Anfang der 1950er Jahre ca. $4 \%^{2}$ (INE 2002, 2004). Sicherlich wird der Export von Gas in naher Zukunft, nach Schaffung einer entsprechenden Transport- und Produktions-infrastruktur, eine deutlich wichtigere Rolle in der Handelsbilanz spielen. Pipelines nach Brasilien und Argentinien existieren bereits, weitere sind in Planung. Diese durchqueren zum großen Teil intakte Waldökosysteme ohne vorhandenes Straßennetz und werden vermutlich als Pioniertrassen einer weiteren Besiedlung genutzt (IBISCH et al. 2002).

Die Wachstumszahlen verdeutlichen, mit welcher Dynamik sich der Wirtschaftsraum Santa Cruz in den letzten Jahrzehnten entwickelt hat. Es liegt auf der Hand, dass Umwälzungen solchen Ausmaßes immer mit einem erheblichen Druck auf die natürlichen Ressourcen verbunden sind. Das zeigt sich am Beispiel des Agrarsektors, dessen Expansion vor allem seit der Einführung von Soja, in kürzester Zeit zu einem erheblichen Rückgang der natürlichen Vegetation zugunsten landwirtschaftlicher Nutzfläche geführt hat (Kap.4.2.3). Zwar hat auch die Entwaldung durch Kleinbauern einen bestimmten Anteil daran, doch entfällt der weitaus größte Flächenanteil der jüngeren Entwaldungen auf technisierte und industrielle landwirtschaftliche Aktivitäten (Kap.4.2.4). Große und mittlere Landwirtschaftsbetriebe expandieren nach wie vor (PACHECO 1998, STEINIGER et al. 2001).

Gegenwärtig werden im Department Santa Cruz mehr als $25.000 \mathrm{~km}^{2}$ für den Anbau landwirtschaftlicher Kulturen verwendet und über $100.000 \mathrm{~km}^{2}$, überwiegend in den natürlichen Graslandschaften der Chiquitania im Osten und des Chaco im Süden, für weidewirtschaftliche Zwecke genutzt (CAO 2002). Die ackerbauliche Nutzung konzentriert sich in der Region um die Stadt Santa Cruz, vornehmlich im Norden und Nordwesten und expandiert seit Ende der 1980er Jahre, insbesondere für großflächigen mechanisierten Anbau, nach Osten in die Region der Chiquitania (Kap.2.3). Diverse Autoren bescheinigen der Region aufgrund der Dominanz junger, fruchtbarer Alluvialböden ein hohes landwirtschaftliches Nutzungspotenzial (Kap.2.2.3), das allerdings z.T. strengen Einschränkungen unterliegt (BARBER 1995, DAVIES 1996, Gerold 1986, GUAMAN 1990). Untersuchungen von GUAMAN (1990) zufolge sind nur ca. 3 \% der Böden im Department ohne größere Restriktionen nutzbar, während 38 \% nur dann langfristig Erträge garantieren, wenn bodenschonende Landnutzungssysteme zum Einsatz kommen. Knapp $50 \%$ sollten, wenn überhaupt, auf weide- und forstwirtschaftliche Nutzungen beschränkt bleiben und $9 \%$ nicht genutzt werden. Noch enger liegen die Grenzen des staatlichen Bodennutzungsplans PLUS (Plan de Uso de Suelo), nach dem nur $12 \%$ der Böden für einen intensiven Anbau in Betracht gezogen werden sollten, während $30 \%$ für Weide- und $32 \%$ für Forstwirtschaft geeignet sind. Der Rest sollte Schutzzone bleiben (URIOSTE \& PACHECO 2001).

Im internationalen Vergleich bewegen sich die bolivianischen Exporte auf einem eher niedrigen Niveau, eine Tatsache die gleichwohl zu der Erkenntnis führt, dass die natürlichen Ressourcen zur Erzielung maximaler Erträge zwangsläufig ökonomischen Interessen

\footnotetext{
2 offizielle Berechnungen wurden erst ab 1965 durchgeführt; Angabe aus MONHEIM \& KÖSTER (1982)
} 
untergeordnet sind. Dieser Logik folgend kommen Landnutzungssysteme zum Einsatz (konventionelle Bodenbearbeitung), die weder an die besonderen klimatischen Bedingungen der Region, noch an die spezifischen Bodenverhältnisse angepasst sind (Kap.2.3.2). Das hat zur Folge, dass viele der ursprünglich fruchtbaren Böden bereits nach wenigen Jahren intensiver ackerbaulicher Nutzung mittlere bis schwere Degradationsschäden aufweisen (Kap.5.1) (BARBER 1995, GEROLD 2001, 2002a).

Die mit zunehmender Bodendegradation sinkende Produktivität annueller Kulturen bedroht die Versorgung des Binnenmarktes ebenso wie den Export. Die fast schon logische Konsequenz aus diesem Dilemma, ist die Rodung neuer Flächen (Kap.4), bzw. auf der Ebene der Kleinbauern die Verkürzung der Brachezeiten (THIELE 1995). Zwar sind in jüngster Zeit vereinzelt Tendenzen erkennbar, bodenschonende Bearbeitungstechniken einzuführen (reduzierte Bearbeitung, Direktsaat), dennoch ist eine Expansion landwirtschaftlicher Nutzflächen in Anbetracht ökonomischer Zwänge solange wahrscheinlich, bis alle potenziell kulturfähigen Böden in Nutzung genommen sind. Die Ausweitung wird mutmaßlich gen Osten erfolgen, da im Süden die strengen klimatischen Bedingungen und im Norden die armen Böden des brasilianischen Schildes einen produktiven Anbau limitieren (STEININGER 2001, URIOSTE \& PACHECo 2001). Diesen Trend vermuteten DinERSTEIN et al. (1995) bereits Mitte der 1990er Jahre und stuften die Region aufgrund ihres hohen biologischen Wertes als die meist gefährdete in den Neotropen ein. 


\subsection{Zielsetzung}

Die vorliegende Dissertation gibt die Ergebnisse eines von der Deutschen Forschungsgemeinschaft (DFG) finanzierten Projekts zur Waldkonversion und Bodendegradation im tropischen Tiefland von Ostbolivien wieder (Laufzeit: 09/2001-12/2005). Das engere Untersuchungsgebiet erstreckt sich auf die Hauptlandwirtschaftszone im Department Santa Cruz. Die Arbeit wurde in Kooperation mit der Fundación Amigos de la Naturaleza (FAN) in Santa Cruz, Bolivien realisiert und bildet eine Fortsetzung der seit vielen Jahren in Bolivien durchgeführten Forschungsarbeiten der Abteilung Landschaftsökologie des Geographischen Instituts der Universität Göttingen. In dem Projekt wurde zudem eine Diplomarbeit über die Niederschlagsvariabilität und das damit verbundene potenzielle Ertragsrisiko in der Projektregion angefertigt (KEMP 2005).

Der Zielgedanke des Projektes und damit vorliegender Arbeit besteht in der Quantifizierung und räumlichen Analyse der in der Untersuchungsregion maßgeblichen Bodendegradationsprozesse, die als Folge der Konversion natürlicher Vegetation in landwirtschaftliche Kulturflächen in den letzten Jahren in verstärktem Maße auftreten. Bei diesen handelt es sich nach BARBER (1995) speziell um Verluste organischer Bodensubstanz und Bodenverdichtungen, aber auch um Bodenerosion durch Wind. Die in diesem Kontext angewendete Methodik soll über die Inwertsetzung vorhandener Bodendaten, zeitaufwändige und kostspielige Bodenuntersuchungen vermeiden. Ein Hauptproblem besteht demnach in der oft geringen Verfügbarkeit von Daten in tropischen Ländern, woraus sich die folgende Fragestellung ableitet:

- Lassen sich für ein vergleichsweise großes Gebiet allein aus vorhandenen, qualitativ heterogenen Bodendaten geringer räumlicher Auflösung unter Einbezug sekundärer Geodaten und Angaben aus der Literatur Bodendegradationsprozesse regional quantifizieren?

Die Beantwortung der Fragestellung impliziert die nachfolgend aufgeführten Teilziele:

- Analyse der Landnutzungsdynamik der letzten zwei Jahrzehnte, der Zeitraum mit den massivsten Einschnitten in das natürliche Landschaftsbild. Diesbezüglich von besonderem Interesse ist die Identifikation von kontinuierlich kultivierten Bereichen und solchen, die eine Umwandlung in Weidenutzung erfahren haben. Letztere können wegen der speziellen Rahmenbedingungen im Untersuchungsgebiet bereits als Indiz abnehmender Bodenfruchtbarkeit gedeutet werden. Mögliche Veränderungen von Bodeneigenschaften unter kontinuierlicher Weidenutzung werden in der Studie nicht quantifiziert.

- Nachgegangen wird der Frage, ob zwischen Relief- und Klimavariablen Zusammenhänge mit der räumlichen Ausprägung natürlicher Bodeneigenschaften bestehen und ob diese für 
eine räumliche Vorhersage von Bodeneigenschaften herangezogen werden können. Dem Vorgehen liegt die Absicht zugrunde, Bodenparameter flächenhaft zu generieren, um diese nachfolgend mit der Landnutzungsinformation zu koppeln.

- Klärungsbedarf besteht bezüglich der Größenordnung und zeitlichen Entwicklung potenzieller Veränderungen von pedoökologischen Parametern (organische Substanz Lagerungsdichte) unter konventioneller Bodenbearbeitung sowie allgemein der Bodenerosionsgefährdung durch Wind. In diesem Zusammenhang stellt sich die Frage, ob im Vergleich der Landnutzungssysteme unterschiedliche Tendenzen zu beobachten sind.

\subsection{Forschungsstand}

\section{Bodendegradation}

Eine qualitative Abschätzung über Ausdehnung, Intensität und Ursachen der weltweit anthropogen verursachten Bodendegradation liefert die von OLDEMAN et al. (1991) publizierte GLASOD Studie (Global Assessment of Soil Degradation). In dieser wurden auf nationaler Ebene mittels einheitlicher Leitlinien (Expertensystem) Informationen über die vorherrschenden Degradationstypen erhoben und zu einer Weltkarte der Bodendegradation (Maßstab $1: 10$ Mio.) zusammengefasst. Einen umfassenden Überblick über Ziele und Erhebungsmethoden des GLASOD Projektes gibt Morgenroth (1999) in ihrer Dissertation zum Stand der Bodenerosion in Entwicklungsländern. Weitere qualitative Ansätze für eine großskalige Abschätzung der Bodendegradation auf Basis von Expertensystemen, wurden mit dem ASSOD Projekt (Assessment of the Status of Human-Induced Soil Degradation) für den südund südostasiatischen Raum (VAN LYNDEN \& OLDEMAN 1997), bzw. mit dem SOVEUR Projekt (Soil Vulnerability Assessment) in Zentral und Osteuropa durchgeführt (VAN LYNDEN 2000). SONNEVELD (2003) vergleicht in einer in Äthiopien durchgeführten Studie qualitative Abschätzungen von Bodenabträgen mit modellierten und kommt zu dem Ergebnis, dass die Expertensysteme dazu neigen Abtragsraten zu überschätzen. Indes liegt ein Vorteil von Expertensystemen in der vergleichsweise schnellen Abschätzung potenzieller Bodendegradation insbesondere für größere Gebiete, wohingegen die getroffenen Aussagen generell subjektiven Charakter haben. Eine GIS-basierte Methodik zur Abschätzung der Bodendegradation unter Wertung verschiedener Einflussgrößen entwickelten ZURAYK et al. (2001). Die Vor- und Nachteile qualitativer Ansätze werden ausführlich bei VAN LYNDEN et al. (2004) diskutiert.

Das Spektrum von Landnutzungssystemen in den Tropen reicht von traditionellen Methoden bis hin zu hochgradig mechanisierten Anbautechniken (LAL 1995). Intensive Bodenbearbeitung führt, wie eingangs erwähnt, zu einer Verschlechterung der Bodeneigenschaften und damit zu einer Abnahme der Produktivität. Sinkende Gehalte organischer Substanz gelten als ein Indikator für diesen Prozess, dies umso mehr, da diese andere wichtige 
Bodeneigenschaften (Aggregatstabilität, KAK, Wasserspeicherkapazität etc.) beeinflussen (BURle et al 1997, CRASWELl \& LEFroy 2001). Dabei treten die höchsten Verluste innerhalb der ersten Anbaujahre auf (DAVIDSON \& ACKERMAN 1993, SOlOMON et al. 2000, ZHAO et al. 2005). Die Kohlenstoff- und Stickstoffdynamik im Boden nach der Konversion natürlicher Vegetation in landwirtschaftliche Nutzflächen, hauptsächlich aus dem tropischen und subtropischen Raum, wird ausführlich im Review von MURTY et al. (2002) diskutiert. Ihren Angaben zufolge sind bei ackerbaulicher Nutzung deutliche C- und N-Verluste zu verzeichnen, während sich unter Weide über alle betrachteten Zeiträume im Durchschnitt keine signifikanten Änderungen zu den Ausgangsgehalten ergeben. Zu vergleichbaren Ergebnissen kommen MCGRATH et al. (2001), die Studien aus dem Amazonasraum hinsichtlich potenzieller Folgen von Landnutzungsänderungen auf die Nährstoffdynamik analysiert haben. GUO \& GIFFORD (2002) ermitteln in ihrem Review eine mittlere Abnahme der C-Vorräte im Oberboden nach Konversion von Wald in eine ackerbauliche Nutzung um annähernd die Hälfte. Die hohen Verluste an organischer Substanz unter ackerbaulicher Nutzung wird als der kritischste Faktor hinsichtlich des Erhalts der Bodenfruchtbarkeit in den Tropen angesehen (GEROLD 1991c, KATYAL et al. 2001, SiVAKUMAR \& VALENTIN 1997). Über die Bedeutung der organischen Substanz in der tropischen Landwirtschaft berichteten z.B. CRASWELL \& LEFROY (2001) und KATYAL et al. (2001).

Nährstoffverluste durch landwirtschaftliche Nutzung sind mehrheitlich Folge der Extraktion von Biomasse (Ernte), Verflüchtigung (Brand), Auswaschung und Erosion (SYERS 1997). Umfassende Untersuchungen über die Veränderung chemischer und physikalischer Bodeneigenschaften unter unterschiedlichen Landnutzungssystemen wurden von LAL (1996a,b,c) in Nigeria durchgeführt. Kontinuierlicher Anbau ohne zusätzliche Düngergaben führt innerhalb kurzer Zeiträume (MCALISTER et al. 1998, THOMAS \& AYARZA 1999) zu einer Verarmung des Bodens an Nährstoffen (JAIYEOBA 2003, LAL 1998). Dieser Prozess ist eng an die Abnahme der organischen Substanz gekoppelt (BRAIMOH \& VLEK 2004, ENEJI et al. 2003). Stoorvogel \& SMALiNG (1998) haben die Ergebnisse lokaler Untersuchungen zur Nährstoffabnahme unter unterschiedlichen Landnutzungssystemen in den Tropen auf größere räumliche Skalen aggregiert (Plot $>$ Farm $>$ Region $>$ Sub-Kontinent). Bis zur Region liefert ihr Ansatz plausible Ergebnisse, darüber hinaus treten methodische Probleme auf, die sie zum großen Teil auf die geringe Datenverfügbarkeit in tropischen Ländern zurückführen.

Bodenverdichtung stellt sich hauptsächlich als Problem infolge mechanischer Belastungen durch landwirtschaftliche Nutzung (HARTEMINK 1998, LAL 1998, PHIRI et al. 2001, Roth et al. 1988), bzw. fehlerhafter Rodungstechniken dar (ENEJI et al. 2003, HAJABBASI et al. 1997, SAHANI \& BEHERA 2001). Kontinuierlicher, monokulturartiger Anbau verstärkt den Effekt durch die geringere Rotation der Anbaukulturen sowie durch häufiges Befahren, bspw. zur Unkrautkontrolle (THOMAS \& AYARZA 1999). Neben Auswirkungen auf bodenphysikalischen Eigenschaften (ANIKWE et al. 2003, ARAUJO et al. 2004, BRONICK \& LAL 2005), hat die Verdichtung des Bodens gleichzeitig rückgekoppelte Effekte auf bodenchemische 
Eigenschaften (BLUMFIELD et al. 2005, KAYOMBO \& LAL 1994). Über den Anstieg der Lagerungsdichte unter Weidenutzung berichten MCGRATH et al (2001) und TownSEND et al. (2002).

Der Aufbruch von Aggregaten infolge von Bodenbearbeitung macht den Boden anfällig für Erosion (CERDÁ 2000, TABOADA et al. 2004). Während Studien zur Erosion durch Wasser als Folge landwirtschaftlicher Nutzung in den Tropen zahlreich sind (BLASCHKE et al. 2000, CAstro et al. 1999, TenBerg et al. 1998), sind diese bezüglich Winderosion gering (BIElders et al. 2000, Dong et al. 2000, Michels et al. 1998) bzw. aus dem tropischen Südamerika nicht bekannt. Hinsichtlich der Qualität der Erosionsforschung in diesen Breiten stellt LAL (2000) fest: ,...the available research information at plot and hillside scale remains ambiguous, incomplete, inconclusive and incredible. [...] It is not the quantity but the quality of available data that is a major constraint to making progress in soil erosion management." Wesentliche Schwächen bestehen seiner Ansicht nach in einem Mangel an standardisierten Untersuchungsmethoden sowie in fehlenden Basisinformationen und ungenügenden Datensätzen vieler Studien.

Umfangreiche Bodenuntersuchungen im Raum Santa Cruz wurden insbesondere in den 1990er Jahren vom Centro de Investigación Agrícola Tropical (CIAT) in Santa Cruz durchgeführt. Im Fokus standen speziell die durch den mechanisierten Anbau verursachten Veränderungen von Bodeneigenschaften (BARBER et al. 1989, BARBOSA et al. 1989, BARBER \& Díaz 1994, Barber \& Romero 1994, BArber 1994, Barber \& NAVArro 1994a,b, BARBer 1995, BARBer et al. 1996, Thiele \& BARBer 1998). Zudem existieren diverse Studien von Gerold (1986, 1991a,b,c, 2001a,b, 2002), die sich mit den Veränderungen bodenphysikalischer und bodenchemischer Parameter unter traditionellen und mechanisierten Landnutzungssystemen beschäftigen.

\section{Bodenregionalisierung}

In der Literatur gibt es verschiedene methodische Ansätze, die räumliche Variabilität von Bodeneigenschaften zu regionalisieren (GOOVAERTS 1999, HEUVELINK \& WEBSTER 2001, MCBRATNEY et al. 2003). Fast alle Verfahren implizieren eine räumliche Schätzung von Punktdaten und müssen wegen der hohen räumlichen Variabilität von Bodeneigenschaften als Abstraktion von der Realität angesehen werden (BÖHNER \& KÖTHE 2003, BURROUGH \& YATES 1994).

Ein herkömmlicher Weg, Informationen über die räumliche Verteilung von Bodeneigenschaften $\mathrm{zu}$ erhalten, besteht in der Ableitung diskreter Flächen in Form von konventionellen Bodenkarten. Dieses Verfahren ist je nach Gebietsgröße zeitaufwändig und in der Regel mit hohen Kosten verbunden (SOMMER et al 2003). Das erklärt, warum ältere Studien vornehmlich auf kleiner Skala durchgeführt wurden bzw. Transsekte untersuchten (SCHLOEDER et al. 2001). Mit Hilfe geostatistischer Methoden und dem Einsatz von 
Geoinformationssystemen (GIS) haben sich Techniken etabliert, die sekundäre Geofaktoren (z.B. Relief, Klima) als Schätzvariablen von Bodeneigenschaften einsetzen (BöHNER et al. 2004, HeuvelinK \& PeBesma 1999). Weitaus am häufigsten werden Bodeneigenschaften über ihre Lage im Relief bestimmt, weniger geläufig ist die Nutzung des Ausgangssubstrats, klimatischer Variablen oder der Vegetation (MCBRATNEY et al. 2003). Räumliche Interpolationsmethoden erlauben eine Vorhersage von Bodeneigenschaften auch für große Gebiete, wie HENDERSON et al. (2004) auf Basis von Klima- und Reliefparametern in einer Studie für ganz Australien darlegen.

Zahlreiche Autoren nutzen lineare Regressionsmodelle, um Bodeneigenschaften auf Basis morphologischer Parameter zu regionalisieren (GESSLER et al. 1995, MCKENZIE \& AUSTIN 1993, MoORE et al. 1993, ODEH et al. 1994, 1995). Grundlage dieser Ansätze ist die Korrelation einer Bodeneigenschaft mit einer unabhängigen Variablen. Die Übertragung in die Fläche erfolgt über einfache oder multiple Regressionen bzw. über Regressionsbäume. Andere nutzen für die Ableitung einer robusten Schätzfunktion zusätzlich Variablen wie Vegetation (McBratney et al. 2000, PARK \& VleK 2002, Skidmore et al. 1997), Ausgangssubstrat (MCKENZIE \& RYAN 1999) und klimatische Parameter (RYAN et al. 2000).

Daneben existieren geostatistische Methoden, Bodeneigenschaften über Kriging Verfahren $\mathrm{zu}$ interpolieren. Ordinary und Universal Kriging liegt entsprechend der Bodenfunktionsgleichung von JENNY (1941) ${ }^{3}$ die Annahme zugrunde, dass sich räumliche Verteilungen mit demselben Muster fortsetzen (BÖHNER \& KÖTHE 2003, BISHOP \& MCBRATNEY 2001, CERRI et al. 2004a, SCHLOEDER et al. 2001). Werden Zusatzvariablen in das Schätzverfahren einbezogen, kommen Co-Kriging (MCBRATNEY et al. 2000) und External Drift Kriging (BOURENNANE et al. 2000) zur Anwendung. Nach Untersuchungen von HeNGL et al. (2004), KALIVAs et al. (2002), KNOTTERS et al. (1995) und ODEH et al. (1994, 1995) werden durch hybride Verfahren, d.h. einer Kombination aus Kriging mit Regressionsmethoden (Regression Kriging), deutlich bessere Vorhersagen von Bodeneigenschaften erzielt, als wenn diese jeweils unabhängig voneinander durchgeführt werden. BöHNER et al. (2002) entwickelten einen Ansatz der bei der Regionalisierung von Bodeneigenschaften stärker die für die Bodenausbildung relevanten Prozessparameter berücksichtigt.

\section{Waldkonversion}

Anthropogen induzierte zeitliche Veränderungen tropischer Landschaftsstrukturen sind Gegenstand zahlloser Untersuchungen und internationaler Programme [z.B. Land Cover Land Use Change (LCLUC), International Geosphere Biosphere Programme (IGBP), Tropical Ecosystem Environment Observation by Satellites (TREES), Large Scale BiosphereAtmosphere Experiment, Amazonia (LBA)]. Im Zentrum des Interesses steht die Analyse der

\footnotetext{
3 nach JENNY (1941) ist die Ausprägung eines Boden als Funktion von Klima, Organismen, Relief, Ausgangsmaterial und Zeit anzusehen.
} 
Konversion tropischer Wälder und Grasländer in landwirtschaftliche Nutzflächen auf unterschiedlichen räumlichen Skalen unter Nutzung von Satellitendaten und GIS-Techniken; lokal (PAN et al. 2004), regional (BARROs FERRAZ et al. 2005, LAAKE et al. 2004), national (MAS et al. 2004, SÁNCHEZ-AzOFEIA et al. 2001), sub-kontinental (CARDILle \& FOLEY 2002) und global (ACHARD et al. 2002, LOVELAND et al. 1997, HANSEN et al. 2000). Über die unterschiedliche Qualität räumlicher Auflösungen berichten KOK \& VELDKAMP (2001). Methoden der räumlichen Erfassung von Landnutzungsänderungen (change detection) auf Basis von Landsat-TM Daten vergleichen READ \& LAM (2002) sowie speziell für das tropische Südamerika TUCKER \& TOWNSHEND (2000). Einige Studien nutzen landwirtschaftliche Zensusdaten für eine Verbesserung der aus Satellitenbildern abgeleiteten Landnutzungsklassen (CARDille \& Foley 2003, Woods \& Skole 1998). Diese Methode ist insbesondere dort sinnvoll, wo über Fernerkundungsdaten keine eindeutige Trennung zwischen Landbedeckungen erzielt werden kann. Die maßgeblichen Ursachen (driving forces) von Landnutzungsänderungen werden ausführlich bei LAMBIN et al. (2001) und GEIST \& LAMBIN (2001) diskutiert.

Räumliche Erklärungsmodelle (spatially explicit models) verwenden die Ursachen potenzieller Flächenkonversionen als unabhängige Variablen, die für die Feststellung einer möglichen Relation meist über Regressionsansätze mit einer abhängigen Variablen (Landnutzung, Vegetation) in Beziehung gesetzt werden (CHOMITZ. \& GRAY 1996). Die Intention solcher Modelle ist, über die Verbindung von ökonomischen (z.B. Bevölkerungsdichte, Marktdistanz, Landeigentum) und biophysikalischen Parametern (z.B. Niederschlagsmenge, Bodenqualität, Topographie), Erkenntnisse über die Gründe vergangener Flächenkonversionen zu gewinnen und Prognosen zukünftiger Landnutzungs- und Vegetationsänderungen zu treffen (MÜller 2003, Verburg et al. 2002, VerburG \& VeldKAMP 2001). Für die Ableitung von Landbedeckungsklassen kommen gewöhnlich Satellitendaten zum Einsatz, die in GIS-Systemen mit den mutmaßlich erklärenden Variablen räumlich verknüpft werden (NELSON \& GEOGHEGAN 2002). Umfangreiche Reviews zu diesem Thema finden sich bei IRWIN \& GEOGHEGAN (2001) und speziell für tropische Gebiete bei KAIMOWITZ \& ANGELSEN (1998). Letztgenannte Autoren kommen in ihrer sehr ausführlichen Analyse zu dem Schluss, dass derartige Modelle nur bis zur Region, allenfalls auf nationaler Ebene anzuwenden sind. Darüber hinaus limitiert die für diese Zwecke schlechte Qualität der Daten und die in der Regel geringe Verfügbarkeit derselben eine sinnvolle Anwendung.

Im tropischen Tiefland von Bolivien wurden mehrere Studien zur Waldkonversion durchgeführt. STEININGER et al. (2001a) analysierten für den Zeitraum von 1984 bis 1994 anhand von Landsat Daten die Entwaldungsrate im bolivianischen Amazonasraum. Ihren Ergebnissen zufolge konzentriert sich mehr als die Hälfte der festgestellten Entwaldung auf die Hauptlandwirtschaftszone des Departments Santa Cruz, dies vor allem als Folge der in den 1990er Jahren stark expandierenden agroindustriellen Landwirtschaft. Die Region um Santa Cruz stand im Fokus einer entsprechenden Untersuchung (1975-1998), die in dem 
vergleichsweise begrenzten Untersuchungsraum (ca. $20.000 \mathrm{~km}^{2}$ ) Entwaldungsraten ermittelte, die Ende der 1990er Jahre mit zu den höchsten weltweit gehörten (STEININGER et al. 2001b). Ebenfalls auf Grundlage von Landsat Daten untersuchten CAMACHO et al. (2001) die Entwicklung der Abholzung im Department Santa Cruz in den 1990er Jahren. Sie kommen zu dem Schluss, dass mehr oder weniger alle für eine landwirtschaftliche Nutzung geeigneten Flächen im Department bereits gerodet sind.

Die Landschaftsentwicklung in den kleinbäuerlichen Kolonisationsgebieten nordwestlich von Santa Cruz beschreiben Millington et al. (2003) für den Zeitraum von 1986-2000 mittels quantitativ-deskriptiver Landschaftsindizes. In der gleichen Region analysierte LozA (2004) die Abholzung und deren treibende Kräfte zwischen 1986-2002 mit Hilfe eines räumlichen Regressionsmodells. Analoge Untersuchungen wurden von VANCLAY et al. (1999), KAIMOWITZ et al. (2002) und MERTENS et al. (2004) für die Landwirtschaftszone (v.a. Agroindustrie) um Santa Cruz durchgeführt. Alle Studien kommen zu der wenig erstaunlichen Erkenntnis, dass die Nähe zu Märkten, Straßen und Bahnlinien ebenso wie Klima, Boden und Kolonisationsgebiete sensitive Parameter einer möglichen Abholzung sind. Die historische Entwicklung der Waldkonversion und deren sozioökonomische Ursachen werden ausführlich bei PACHECO (1998) beschrieben.

BounOUA et al. (2003) untersuchten unter Nutzung des Biosphären Modells SiB2 den Einfluss der zunehmenden Waldrodung auf das lokale Klima im Raum Santa Cruz. Ihren Ergebnissen nach liegen im Vergleich zur natürlichen Vegetation die Temperaturen auf Anbauflächen im Schnitt um $0,6^{\circ} \mathrm{C}$ bis $1,2^{\circ} \mathrm{C}$ höher. Bezogen auf die Region bedeutet das eine geringe Erhöhung der mittleren Monatstemperaturen; ein Effekt, der über Messwerte des nationalen Wetterdienstes SENAMHI Bestätigung findet. 


\section{DAS UNTERSUCHUNGSGEBIET}

\subsection{Einführung}

Das Department Santa Cruz nimmt mit $370.000 \mathrm{~km}^{2}$ ca. 33,7 \% der Landesfläche Boliviens ein (Abb.2.1). Es ist das Größte der neun Departments Boliviens (MONTES DE OCA 1997). Begrenzt durch den brasilianischen Schild im Nordosten und die subandinen Gebirgsausläufer im Südwesten erstreckt sich von Südosten nach Nordwesten eine ausgedehnte Alluvialebene (Chaco- und Benitiefland), die im Department ebenso wie im Untersuchungsgebiet den größten Flächenanteil innehat (Abb.2.2). Das Tiefland bildet die Sedimentationsebene der Anden und des brasilianischen Schildes (Kap.2.2.2), in der sich erdgeschichtlich junge Alluvialböden entwickelt haben (Kap.2.2.3).

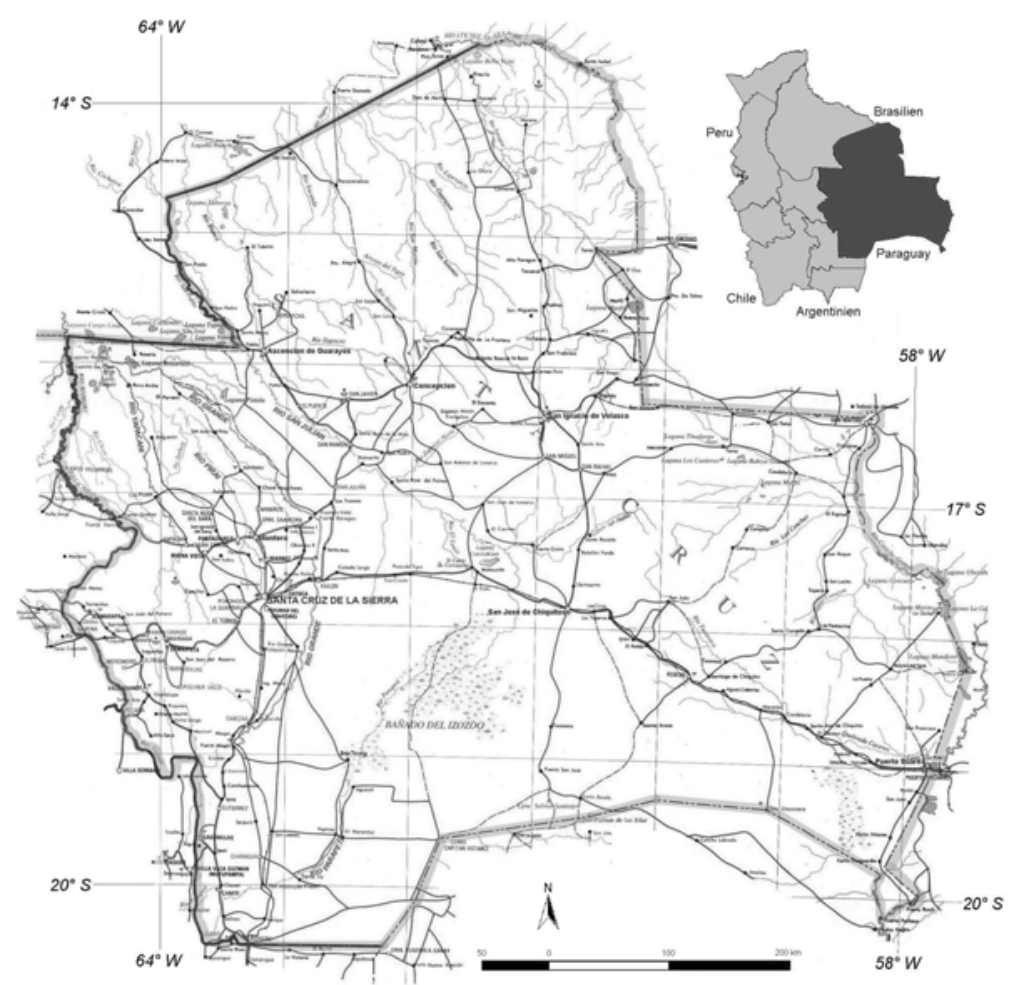

Abb. 2.1: Lage des Department Santa Cruz innerhalb Boliviens Quelle: Instituto Geográfico Militar, Atlas Digital de Bolivia

Die Region liegt in einer Übergangszone zwischen den feuchttropischen Wäldern des Amazonas, dem Cerrado-Savannen Komplex Brasiliens und den Dornbuschwäldern des Gran Chaco (Kap.2.2.4). Die Konvergenz verschiedener biogeographischer Zonen hat in Ostbolivien ein weites Spektrum an tropischen Trockenwäldern, halbimmergrünen Regenwäldern, Savannen und Feuchtgebieten mit einer hohen biologischen Vielfalt entstehen lassen. Die Chiquitania Region im Osten Boliviens besitzt eines der größten zusammenhängenden tropischen Trockenwaldgebiete weltweit (KILLEEN et. al 1998, MYERS et al. 
2000). Humide Einflüsse aus dem Amazonasbecken und trocken-heiße Bedingungen des Chaco führen $\mathrm{zu}$ einer ausgeprägten regionalen Klimadifferenzierung und einem häufigen Wechsel trockener und feuchter Jahre (Kap.2.2.1). Die landwirtschaftliche Produktion konzentriert sich im wesentlichen auf zwei Zonen, eine ältere Erschließungszone westlich des Río Grande (zentrale Zone) und eine jüngere Expansionszone östlich des Río Grande (Abb.8). Zudem wird in den natürlichen Graslandschaften extensive Weidewirtschaft betrieben (Kap.2.3.2). Das Department Santa Cruz besitzt innerhalb Boliviens das höchste landwirtschaftliche Potenzial.

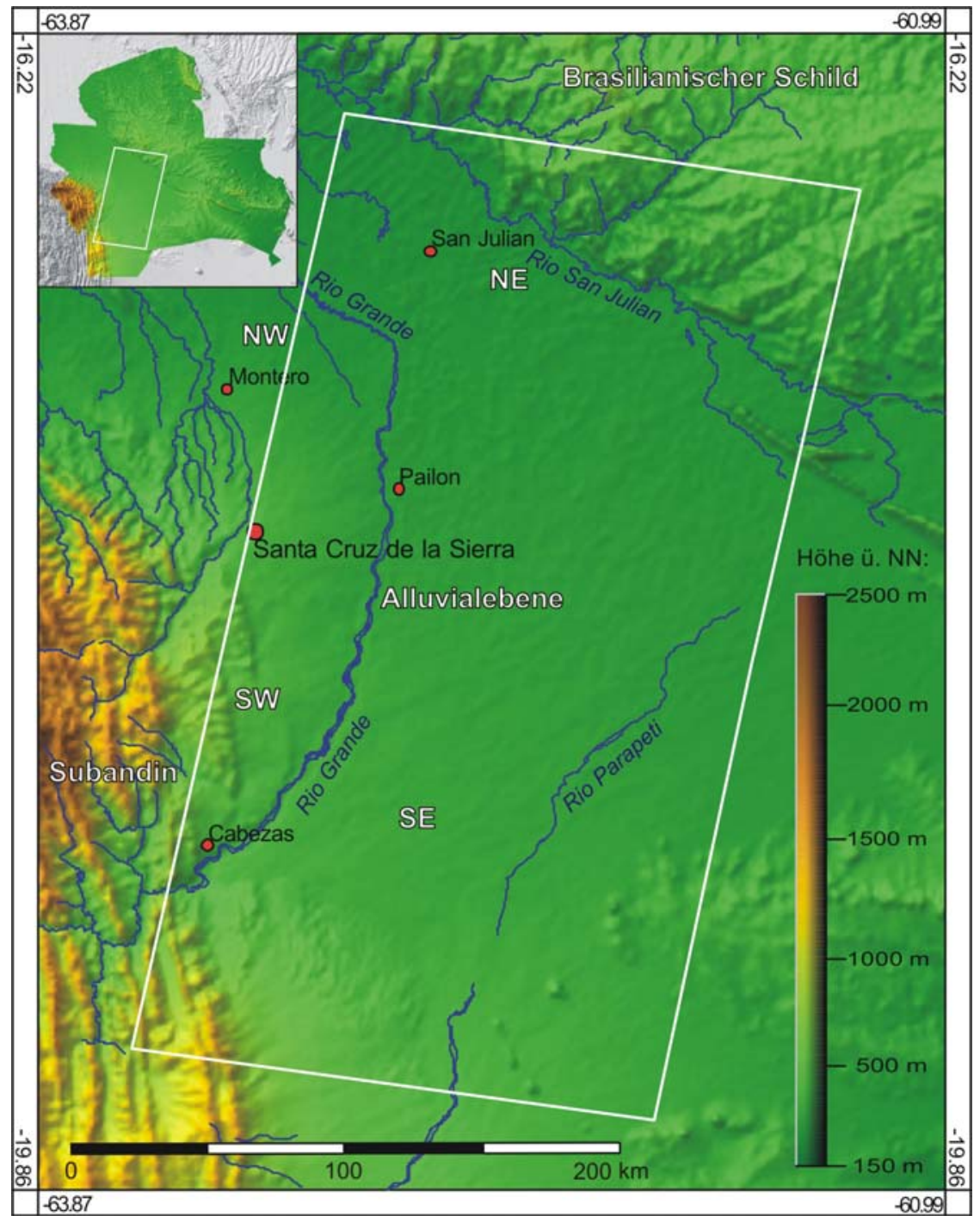

Abb. 2.2: Lage des Untersuchungsgebietes

Das engere Untersuchungsgebiet erstreckt sich ca. $360 \mathrm{~km}$ in N-S und $180 \mathrm{~km}$ in W-E Richtung $\left(16^{\circ} 42^{\prime} \mathrm{S}, 61^{\circ} 15^{\prime} \mathrm{W}-19^{\circ} 24^{\prime} \mathrm{S}, 63^{\circ} 34^{\prime} \mathrm{W}\right)$ und umfasst eine Fläche von knapp 58.000 $\mathrm{km}^{2}$. Die Beschreibung der naturräumlichen Ausstattung folgt einer regionalen Einteilung in subandine Zone, brasilianischer Schild und Alluvialebene (Abb.2.2). 


\subsection{Naturräumliche Ausstattung}

\subsubsection{Klima}

Das östliche bolivianische Tiefland befindet sich in einer klimatischen Übergangszone. Von NW nach SE äußert sich das in tropisch-humiden über tropisch-wechselfeuchte Bedingungen (Aw-Klima n. KOEPPEN) bis zu subtropisch-semiariden Bedingungen im Chacotiefland (BSh-Klima n. KOEPPEN). Kennzeichnend ist eine Abnahme der Niederschläge von etwa $2.000 \mathrm{~mm} \mathrm{a}^{-1}$ auf unter $500 \mathrm{~mm} \mathrm{a}^{-1}$ auf einer Distanz von nur $200 \mathrm{~km}$ (Abb.3). Gleichzeitig nimmt die Dauer der Trockenzeit (Juni-September) zu. 70 \% der jährlichen Niederschlagssumme konzentriert sich auf die Monate Oktober bis März, auf die Trockenzeit entfallen knapp $15 \%$ (Mittelwerte aus 77 Klimastationen).
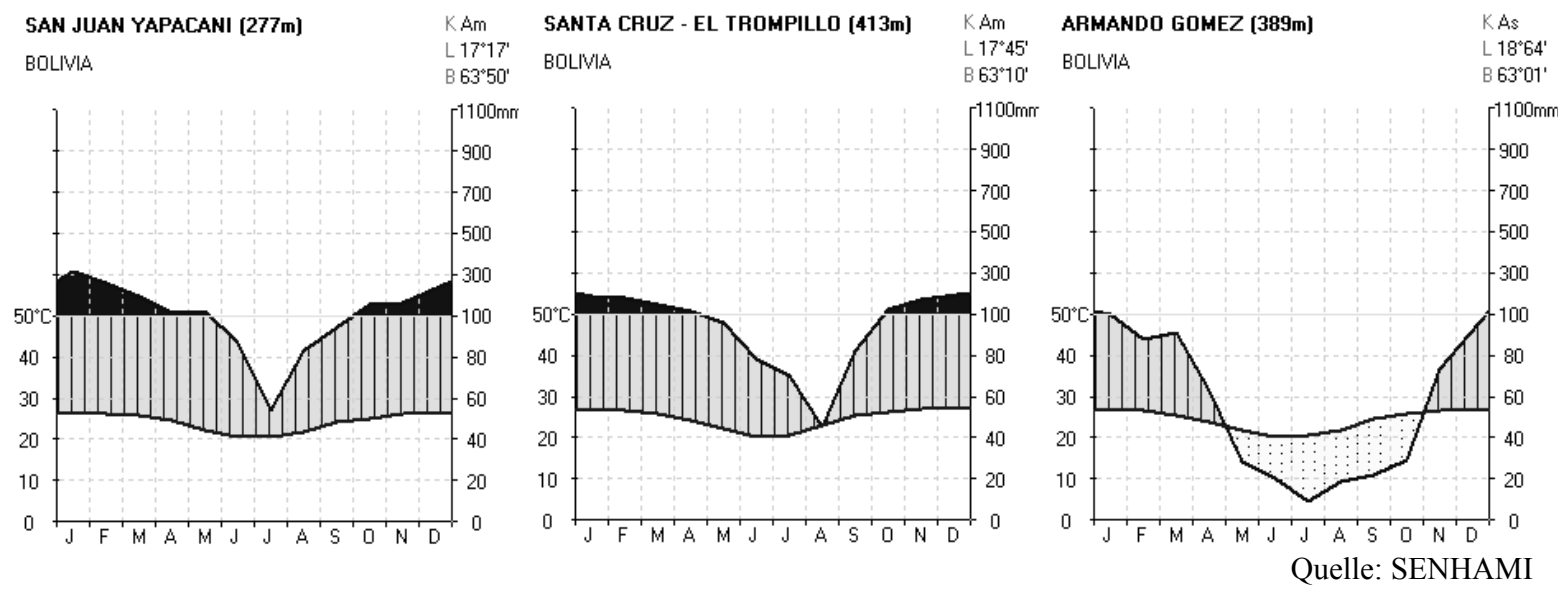

Abb. 3: Klimadiagramme der Stationen San Juan de Yapacani, Santa Cruz und Armando Gomez

Die jährlichen Verdunstungsraten schwanken zwischen 1.000-1.600 mm. Mit zunehmender Breitenlage steigt die potenzielle Verdunstung deutlich. Das hydrologische Defizit beträgt in der nördlichen Chacoregion z.T. mehr als $500 \mathrm{~mm} \mathrm{a}^{-1}$. Bei abnehmenden Niederschlägen und höheren Einstrahlungsintensitäten steigt die Gefahr von Dürren. Ab etwa $18^{\circ}$ südl. Breite treten in der Trockenzeit alle 2-3 Jahre Monate ohne Niederschlag auf. Langanhaltende Trockenperioden treten Schätzungen zufolge alle 7-10 Jahre auf (IADB 2000).

Kennzeichnend für die Niederschläge ist eine auffallende räumliche und zeitliche Variabilität sowie während der Regenzeit hohe Intensitäten und längere Zeiträume ohne Niederschlag. Die Variabilitäten liegen im Mittel bei $17 \%$ und zeigen einen deutlichen Anstieg mit Annäherung an die Anden (Tab.1). Zu gleichen Ergebnissen kommt GEROLD (1986), der diese Größenordnung als für die Randtropen typisch ansieht. Die jährlichen Abweichungen liegen im Mittel bis zu $50 \%$ unter respektive über dem arithmetischen Mittel, im Extremfall höher. 
Ausschlaggebend für die klimatischen Verhältnisse ist die jahreszeitlichen Verschiebung der ITCZ. Im Südsommer liegt die ITCZ zwischen $10^{\circ}$ und $15^{\circ}$ südl. Breite In dieser Zeit führen Nordostpassate feucht-warme Luftmassen äquatorialen Ursprungs heran, die sich an der Andenostabdachung stauen, dort reichlich Niederschläge bringen und als Nordwestwinde in das östliche bolivianische Tiefland abgelenkt werden. Die feuchten Luftmassen bewirken intensive Konvektionsniederschläge. Südlich des 18. Breitengrades wird dieses System zunehmend durch den Einfluss der Südostpassate aus den subtropischen Hochdruckzellen der benachbarten Ozeane überlagert. Besonders in den Sommermonaten zieht das über dem Gran Chaco gelegene Hitzetief die aus dem Atlantikhoch abströmenden Luftmassen tief in den Kontinent hinein und bewirkt eine Erhöhung der Niederschläge am Andenrand (GEROLD 1986, SERVANT et al. 1993). Vor allem im Südwinter, aber auch während der übrigen Monate kommt es wiederholt zu einer Verdrängung der feucht-warmen Luft durch Kaltluftfronten aus dem Süden (Surazos). Dieser Effekt drückt sich in kurzfristigen, deutlichen Temperaturabfällen begleitet von Niederschlägen und starken Winden aus. Die Surazos dauern in der Regel wenige Tage, können aber auch bis zu zwei Wochen das Wetter bestimmen (RONCHAIL 1986).

Tab. 1: Statistische Parameter verschiedener Klimastationen im Department Santa Cruz

\begin{tabular}{|c|c|c|c|c|c|c|c|c|}
\hline Station & Messperiode & lat & long & $\begin{array}{l}\text { Min } \\
(\mathbf{m m})\end{array}$ & $\begin{array}{l}\text { Max } \\
(\mathbf{m m})\end{array}$ & $\begin{array}{l}\text { Mittel } \\
(\mathbf{m m})\end{array}$ & $\begin{array}{c}\text { mittlere } \\
\text { Abweichung (mm) }\end{array}$ & $\begin{array}{c}\text { relative } \\
\text { Variabilität }(\%)\end{array}$ \\
\hline \multicolumn{9}{|l|}{ Alluvialebene } \\
\hline San Julian & $1978-1987$ & $16^{\circ} 67^{\prime}$ & $62^{\circ} 47^{\prime}$ & 970 & 1651 & 1322 & 211 & 16 \\
\hline San Juan Yapacani & $1960-1980$ & $17^{\circ} 25^{\prime}$ & $63^{\circ} 83^{\prime}$ & 1396 & 2881 & 1893 & 296 & 16 \\
\hline Tres Cruces & $1978-2003$ & $17^{\circ} 38^{\prime}$ & $62^{\circ} 14^{\prime}$ & 443 & 1287 & 919 & 158 & 17 \\
\hline Cotoca & $1982-2003$ & $17^{\circ} 45^{\prime}$ & $62^{\circ} 59^{\prime}$ & 717 & 2157 & 1174 & 212 & 18 \\
\hline Santa Cruz (Central) & $1982-2003$ & $17^{\circ} 47^{\prime}$ & $63^{\circ} 10^{\prime}$ & 867 & 2320 & 1502 & 283 & 19 \\
\hline Estación Mora & 1977-1998 & $18^{\circ} 27^{\prime}$ & $63^{\circ} 13^{\prime}$ & 570 & 1444 & 1030 & 202 & 20 \\
\hline Cabezas & $1977-1996$ & $18^{\circ} 47^{\prime}$ & $63^{\circ} 19^{\prime}$ & 507 & 1134 & 838 & 151 & 18 \\
\hline \multicolumn{9}{|l|}{ brasilianischer Schild } \\
\hline Concepción & 1943-1998 & $16^{\circ} 07^{\prime}$ & $62^{\circ} 02^{\prime}$ & 799 & 1831 & 1194 & 170 & 15 \\
\hline San Javier & $1982-1998$ & $16^{\circ} 20^{\prime}$ & $62^{\circ} 38^{\prime}$ & 1053 & 2384 & 1499 & 230 & 15 \\
\hline San Jose de Chiquitos & $1982-1998$ & $17^{\circ} 47^{\prime}$ & $60^{\circ} 47^{\prime}$ & 564 & 1677 & 1135 & 182 & 16 \\
\hline \multicolumn{9}{|l|}{ subandine Zone } \\
\hline Florida & 1978-1999 & $18^{\circ} 34^{\prime}$ & $63^{\circ} 23^{\prime}$ & 501 & 3056 & 1389 & 500 & 36 \\
\hline Abapó & $1977-1998$ & $18^{\circ} 55^{\prime}$ & $63^{\circ} 25^{\prime}$ & 349 & 1751 & 1071 & 286 & 28 \\
\hline \multicolumn{9}{|l|}{ Chaco } \\
\hline Armando Gomez & $1969-1981$ & $18^{\circ} 39^{\prime}$ & $63^{\circ} 01^{\prime}$ & 477 & 825 & 652 & 91 & 14 \\
\hline
\end{tabular}

Die Temperaturen liegen im Jahresverlauf zwischen $26,8{ }^{\circ} \mathrm{C}$ (Dezember) und $21,0{ }^{\circ} \mathrm{C}$ (Juli). Die Amplitude ist mit $7{ }^{\circ} \mathrm{C}$ gering. Im Sommer können Extremwerte von $40{ }^{\circ} \mathrm{C}$, im Winter von $3{ }^{\circ} \mathrm{C}$ erreicht werden (NAVARRO \& MALDONADO 2002). Die relative Luftfeuchte 
schwankt im Jahr zwischen $55 \%$ und $80 \%$, wobei niedrige Werte ausschließlich im Chacotiefland auftreten (AGRAR- \& HYDROTECHNIK 1973).

Angaben über die Windverhältnisse im Untersuchungsraum folgen in Kapitel 5.1.4.1.

\subsubsection{Geologie, Geomorphologie}

Die subandine Zone unterteilt sich in eine gebirgige Zone und eine hügelige Andenfußzone. Die Nord-Süd streichenden Gebirgsketten setzen sich aus marinen Gesteinen des Devon, kontinentalen Sedimenten des Permokarbon sowie des Mesozoikum zusammen (IADB 2000). Die absoluten Höhen liegen bei $2.100 \mathrm{~m}$ ü. NN. Die Andenfußzone befindet sich im Übergangsbereich zur Alluvialebene. In der Region werden maximale Höhen von 600 $m$ ü. NN erreicht. Der Untergrund besteht aus tertiären Sedimenten mit einer Mächtigkeit von bis zu $2.500 \mathrm{~m}$. Die Sedimente sind meist fluviatil zerschnitten und örtlich von pleistozänen Terrassen überdeckt. Die Terrassen zeichnen sich durch leicht verfestigte Sande, Konglomerate sowie Geröll- und Schotterlagen aus (GEROLD 1986, WERDING 1977). In der subandinen Zone befinden sich die Quellgebiete der größeren Flüsse Grande, Parapetí, Pirai und Yapacani sowie zahlreicher kleinerer Flüsse, die im Quartär andine Sedimente in der Alluvialebene akkumuliert haben.

Der tiefere geologische Untergrund der Alluvialebene setzt sich aus marinen Ablagerungen des Silur und Devon zusammen, die in der Folge mit kontinentalen Sedimenten des oberen Karbon, Mesozoikum, Tertiär und Quartär überdeckt wurden. Das rezente Erscheinungsbild wird durch fluviatile, äolische und lakustrine Sedimente des Quartär bestimmt. Seismische Untersuchungen zur Erdölexploration haben in den 1970er Jahren quartäre Ablagerungen von bis $\mathrm{zu} 1.500 \mathrm{~m}$ Mächtigkeit nachgewiesen (IADB 2000). Die fluviatilen Sedimente entstammen überwiegend der subandinen Zone, nur im Nordosten zu einem geringen Teil dem brasilianischen Schild. Die äolischen Sedimente haben ihren Ursprung in den sandigen Uferbereichen der Vorfluter der subandinen Zone, die vor allem im Pleistozän viel Sediment in die Ebene befördert haben, bzw. rezent während der Regenzeit transportieren. Daraus haben sich im Südosten des Río Pirai (Lomas de Arena) und des Río Grande (Arenales de Guanaco) durch die vorherrschenden Nordwestwinde größere Dünengebiete entwickelt. Quartäre Flussverlagerungen haben in der Ebene ein ausgeprägtes Mesorelief geformt. Dabei blieben im Landschaftsbild neben Flussterrassen tote Flussarme und ehemalige Abflussrinnen als natürliche Depressionen zurück, in denen sich z.T. Lagunen ausgebildet haben (z.B. Laguna de Concepción). Diese Senkenbereiche sind durch die Akkumulation sehr feinen Substrats gekennzeichnet, in denen sich während der Regenzeit das Niederschlagswasser staut oder die größere Überschwemmungsbereiche, wie die Bañados del Izozog im Südosten des Untersuchungsgebietes, bilden (GUAMAN 1988a,b, IADB 2000, WERDING 1977). Die Alluvialebene ist demnach durch einen kleinräumigen Wechsel toniger bis sandiger Sedimen- 
te charakterisiert. Räumlich betrachtet findet eine Korngrößensortierung mit zunehmender Entfernung vom Andenrand statt. Überwiegen westlich des Río Grande vielfach sandige Texturen, so bestimmen im Norden und Osten durch ruhigere Sedimentationsbedingungen Schluffe und Tone das Erscheinungsbild. Die Alluvialebene besitzt ein leichtes Gefälle Richtung Nordost (GUAMAN 1988a).

Der altkristalline brasilianische Schild bildet mit seinen südwestlichen Ausläufern die älteste geologische Formation im Untersuchungsgebiet. Dieser setzt sich aus Gneisen, Graniten und Granitoiden des Präkambriums und Sedimentgesteinen des Kambriums zusammen, die partiell intensiv metamorph überformt wurden. Seit Abschluss der letzten gebirgsbildenden Phase vor ca. 550 Mio. Jahren finden intensive Einebnungsprozesse statt. Mittlere Höhen liegen zwischen 500-800 m ü. NN. Das aktuelle Erscheinungsbild ist im Osten durch eine sanft wellige Hügellandschaft mit weiten Ebenen geprägt, während am westlichen Rand ein stärker zerschnittenes Relief mit z.T. engen Tälern dominiert (NAVARRO \& MALDONADO 2002, IPORRE 1996).

\subsubsection{Böden}

Die Beschreibung der Bodentypen erfolgt anhand der Profildaten unter natürlicher Vegetation. Die taxonomische Einordnung richtet sich nach der in Südamerika gebräuchlichen US Soil Taxonomy (SOIL SuRVEy STAFF 2003), die Bewertung chemischer Bodeneigenschaften erfolgt anhand von Richtlinien des Centro de Investigacíon Agrícola Tropical (CIAT) in Santa Cruz (Tab.A1, Anhang). Gleichzeitig werden potenzielle Restriktionen einer landwirtschaftlichen Nutzung der Böden beschrieben.

Die Böden der subandinen Zone ${ }^{4}$ sind je nach Reliefposition geringmächtig (AC-Profil, Kuppen) bis tiefgründig (ABC-Profil, Täler) entwickelt. Im Korngrößenspektrum dominieren Sande und Lehme (Abb.4). Hauptbodentypen sind Inceptisole, sowie in geringerer Verbreitung Alfisole und Entisole. Die Bodenreaktion liegt im schwach sauren Bereich. Die KAK ist gering, die Basensättigung hoch. Die Böden weisen eine mäßige Nährstoffversorgung mit N, P und K auf. Die Gehalte an organischer Substanz (SOM) sind niedrig (Tab.2). Limitierende Faktoren für eine landwirtschaftliche Nutzung sind das stellenweise steile Relief und die damit verbundene Anfälligkeit gegenüber Wassererosion sowie die ungünstige Nährstoffausstattung und geringe Wasserspeicherkapazität der sandigen Böden (GUAMAN 1999).

Die Böden der Alluvialebene sind mehrheitlich tiefgründig entwickelt und weisen deutliche ABC-Profile auf. In Bereichen periodischer Überflutungen ist der B-Horizont z.T. nur schwach ausgeprägt oder fehlt ganz (GUAMAN 1988). Die Böden besitzen überwiegend mittlere bis hohe Nährstoffgehalte. Taxonomisch dominieren Inceptisole und Alfisole, die

\footnotetext{
${ }^{4}$ Die Böden der gebirgigen Zone werden nicht beschrieben, da sie in der Untersuchung nicht von Relevanz sind.
} 
sich in schluffreichem Ausgangsmaterial entwickelt haben. Mit zunehmender Nähe zum Andenrand treten vermehrt nährstoffarme sandige Entisole auf. Die Alluvialebene ist von Altarmen und ehemaligen Abflussrinnen (Depressionen) durchzogen, in denen Tonböden (Vertisole) dominieren. Im Chaco sind zunehmend Aridisole ausgebildet.
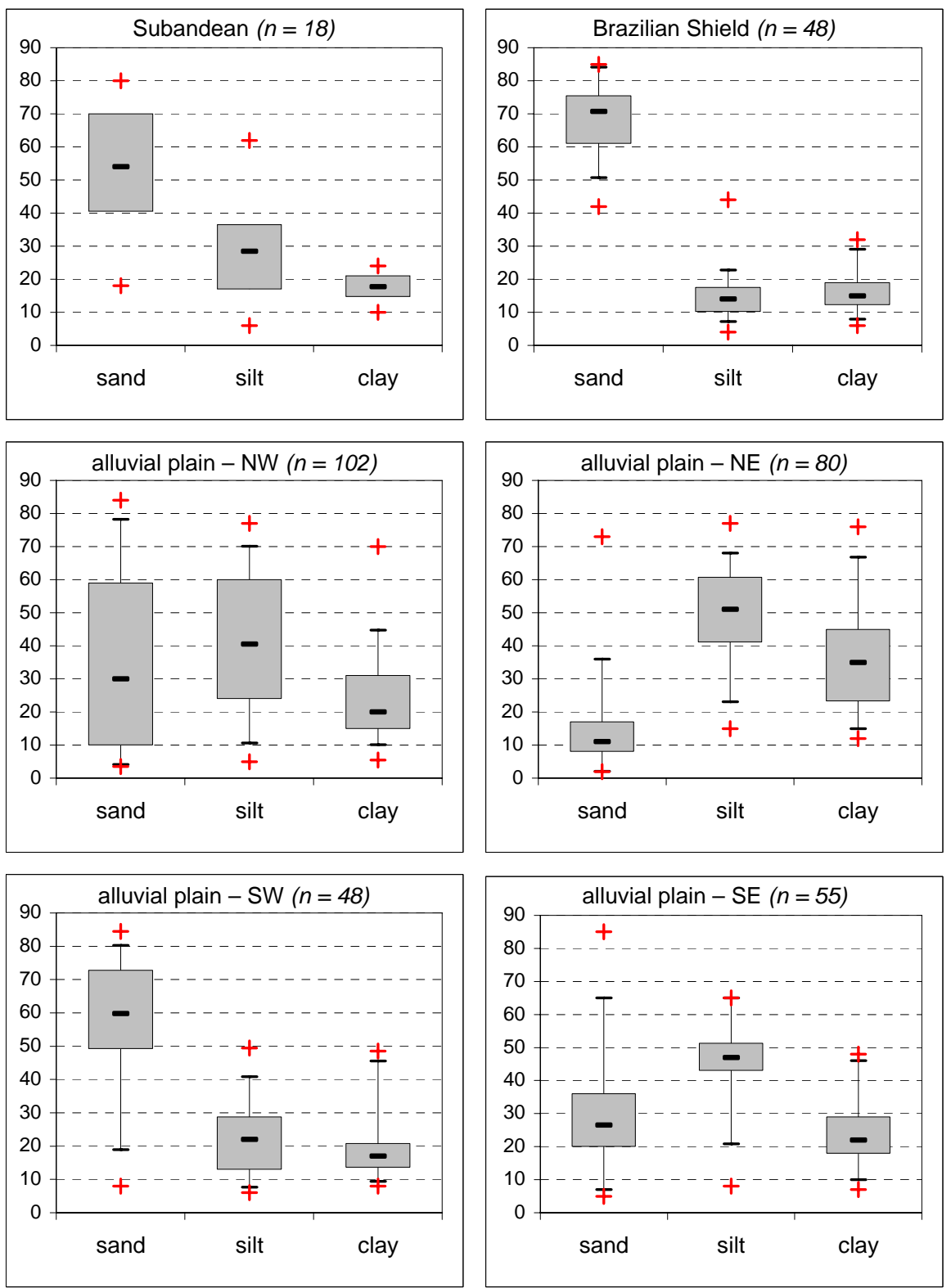

Abb. 4: Mittlere Korngrößenzusammensetzung der Böden im Untersuchungsgebiet (Anm.: Die subandine Zone umfasst nur die Andenfußzone; Quelle: siehe Kap. 3.2)

25. und 75. Perzentil des arithmetischen Mittelwerts

- Median

$\longmapsto$ Spannweite, in der sich $90 \%$ aller Messwerte befinden

$+\quad$ Min, Max 
Die Bodenlandschaft westlich des Río Grande (Alluvialebene NW) ist überaus heterogen. Das Bodenartenspektrum reicht von lehmigen Sanden bis zu reinen Tonen, mit einer leichten Dominanz sandiger Lehme (Abb.4). Die Bodenreaktion befindet sich im schwach sauren bis schwach alkalischen Bereich. Die KAK ist niedrig bis mäßig, die Basensättigung hoch. Entsprechend der Vergesellschaftung von sandigen, schluffigen und tonigen Substraten sind die K-Gehalte niedrig bis hoch, während $\mathrm{P}$ überwiegend Mangelelement ist. Markante regionale Unterschiede zeigen sich auch beim Stickstoff und der organischen Substanz, mit sowohl niedrigen als auch hohen Gehalten (Tab.2). Nutzungseinschränkungen weisen die Entisole (Nährstoffdefizite, geringe Wasserspeicherkapazität) und die Böden mit hohen Tongehalten (geringe Wasserleitfähigkeiten, Staunässeerscheinungen) auf.

Tab. 2: Regionale Einordnung bodenchemischer Parameter in Ostbolivien

\begin{tabular}{|c|c|c|c|c|c|c|c|c|}
\hline & pH * & $\begin{array}{c}\mathbf{K} \\
{\left[\mathrm{cmol} \mathrm{kg}^{-1}\right]}\end{array}$ & $\begin{array}{c}\text { KAK } \\
{\left[\mathrm{cmol} \mathrm{kg}^{-1}\right]}\end{array}$ & $\begin{array}{c}\text { BS } \\
{[\%]}\end{array}$ & $\begin{array}{c}\mathbf{P} * * \\
{\left[\mathrm{mg} \mathrm{kg}^{-1}\right]}\end{array}$ & $\begin{array}{c}\text { SOM } \\
{[\%]}\end{array}$ & $\begin{array}{c}\mathbf{N}_{\mathbf{t}} \\
{[\%]}\end{array}$ & $\mathbf{C} / \mathbf{N}$ \\
\hline \multicolumn{9}{|c|}{ subandine Zone } \\
\hline$n$ & 7 & 7 & 7 & 7 & 5 & 5 & 5 & 5 \\
\hline MW & 5,93 & 0,35 & 9,4 & 97,3 & 5,8 & 2,3 & 0,14 & 10,2 \\
\hline Min & 5,30 & 0,17 & 4,1 & 93,0 & 3,0 & 1,6 & 0,08 & 7,4 \\
\hline Max & 6,92 & 0,67 & 13,4 & 100,0 & 8,0 & 3,2 & 0,25 & 12,2 \\
\hline Stabw & 0,63 & 0,17 & 3,3 & 2,5 & 2,3 & 0,7 & 0,07 & 2,2 \\
\hline \multicolumn{9}{|c|}{ Alluvialebene NE } \\
\hline$n$ & 53 & 51 & 42 & 41 & 42 & 51 & 51 & 51 \\
\hline MW & 5,97 & 0,95 & 14,0 & 94,7 & 22,2 & 3,4 & 0,18 & 11,1 \\
\hline Min & 4,50 & 0,13 & 4,4 & 65,0 & 4,0 & 1,2 & 0,06 & 4,2 \\
\hline Max & 7,90 & 2,28 & 27,2 & 100,0 & 44,0 & 7,1 & 0,36 & 20,0 \\
\hline$\underline{\text { Stabw }}$ & 0,76 & 0,54 & 5,7 & 8,7 & 10,4 & 1,4 & 0,07 & 2,8 \\
\hline \multicolumn{9}{|c|}{ Alluvialebene SE } \\
\hline$n$ & 55 & 51 & 52 & 40 & 38 & 28 & 22 & 22 \\
\hline MW & 6,84 & 0,67 & 12,9 & 93,2 & 15,9 & 2,1 & 0,18 & 7,4 \\
\hline Min & 5,00 & 0,15 & 2,8 & 47,6 & 2,5 & 1,2 & 0,12 & 3,6 \\
\hline $\operatorname{Max}$ & 8,25 & 1,20 & 25,8 & 100,0 & 39,5 & 3,8 & 0,27 & 12,7 \\
\hline Stabw & 0,71 & 0,28 & 5,0 & 10,9 & 9,4 & 0,7 & 0,05 & 2,4 \\
\hline \multicolumn{9}{|c|}{ Alluvialebene NW } \\
\hline$n$ & 27 & 23 & 9 & 7 & 7 & 26 & 22 & 22 \\
\hline MW & 6,50 & 0,59 & 10,3 & 72,6 & 5,7 & 2,9 & 0,16 & 10,3 \\
\hline Min & 5,20 & 0,09 & 2,6 & 28,0 & 0,6 & 1,2 & 0,06 & 4,4 \\
\hline Max & 8,30 & 1,81 & 20,0 & 99,0 & 18,0 & 6,3 & 0,26 & 13,8 \\
\hline Stabw & 0,92 & 0,40 & 5,8 & 27,7 & 6,4 & 1,2 & 0,05 & 2,4 \\
\hline \multicolumn{9}{|c|}{ Alluvialebene SW } \\
\hline$n$ & 13 & 13 & 13 & 13 & 8 & 13 & 13 & 13 \\
\hline MW & 6,54 & 0,70 & 15,6 & 96,4 & 25,7 & 2,3 & 0,22 & 6,1 \\
\hline Min & 5,80 & 0,27 & 6,5 & 83,8 & 7,8 & 1,2 & 0,11 & 3,6 \\
\hline Max & 7,65 & 1,48 & 25,8 & 100,0 & 52,0 & 3,7 & 0,34 & 9,9 \\
\hline Stabw & 0,52 & 0,30 & 6,2 & 4,6 & 16,2 & 0,9 & 0,06 & 2,1 \\
\hline \multicolumn{9}{|c|}{ brasilianischer Schild } \\
\hline$n$ & 40 & 33 & 40 & 34 & 34 & 37 & 37 & 37 \\
\hline MW & 6,07 & 0,29 & 5,6 & 92,1 & 4,9 & 2,7 & 0,19 & 8,4 \\
\hline Min & 4,40 & 0,10 & 1,7 & 53,0 & 1,5 & 1,1 & 0,07 & 6,4 \\
\hline $\operatorname{Max}$ & 7,90 & 0,62 & 16,2 & 100,0 & 20,0 & 5,3 & 0,35 & 14,0 \\
\hline Stabw & 0,68 & 0,14 & 3,3 & 12,1 & 3,2 & 1,1 & 0,08 & 1,8 \\
\hline
\end{tabular}

* gemessen in $\mathrm{H}_{2} \mathrm{O}$, ** Methode Olsen

Anm.: Oberboden, Ausgangsgehalte unter natürlicher Vegetation 
Verschiedene Untersuchungen haben gezeigt, dass auch die relativ gut mit Nährstoffen versorgten Böden ohne Düngerapplikationen bereits nach wenigen Jahren unter Nutzung merkliche Defizite an N, P und K aufweisen (GEROLD 1986, GUAMAN 1990, KIDMAN et. al 1980).

Südlich von Santa Cruz (Alluvialebene SW) überwiegen sandige Lehme mit mittleren Sandanteilen im Oberboden von $60 \%$ (Abb.4). Die Bodenreaktion ist schwach sauer bis schwach alkalisch, bei mittleren Austauschkapazitäten und hoher Basensättigung. Die Nährstoffverfügbarkeit von N, P und K ist hoch, die Gehalte an organischer Substanz gering (Tab.2). Einschränkende Faktoren für eine agrarische Nutzung sind klimatischen Ursprungs. Die häufig auftretenden starken Winde in der Region haben auf den sandigen Lehmen bereits enorme Erosionsschäden nach sich gezogen. Verstärkt wird der Prozess durch geringe Humusgehalte (schwache Bindungskräfte zwischen Einzelkörnern) und ein geringes Wasserspeichervermögen (schnelle Austrocknung) dieser Böden. Zudem ziehen periodisch auftretende Trockenperioden wiederholt Ertragsausfälle nach sich. Die tonigeren Böden der Senkenbereiche besitzen nur geringe Wasserleitfähigkeiten und neigen z.T. zur Versalzung.

Östlich des Río Grande (Alluvialebene NE) verschiebt sich das Korngrößenspektrum in Richtung schluffiger und toniger Lehme (Abb.4). Sandige Bodenarten sind, anders als im Nordwesten der Alluvialebene, weniger verbreitet. Die Bodenreaktion liegt vornehmlich im schwach sauren Bereich. Die KAK ist niedrig bis mäßig. Die Böden sind gut mit Basen versorgt und weisen hohe P- und K-Gehalte auf. Vereinzelt wurden P-Gehalte von über $40 \mathrm{mg}$ $\mathrm{kg}^{-1}$ gemessen $^{5}$ (GUAMAN 1988). Die Gehalte an Stickstoff und organischer Substanz variieren deutlich, befinden sich aber überwiegend auf mittlerem bis hohem Niveau (Tab.2). Diese Region (Expansionszone) besitzt im Department Santa Cruz das höchste Nutzungspotenzial. Nach GUAMAN (1988b, 1990) eignen sich ca. 90 \% der Böden als Kulturflächen, vorrausgesetzt bodenschonende Bearbeitungstechniken kommen zum Einsatz. Insbesondere die schluffreichen Böden sind verdichtungsanfällig und neigen im feuchten Zustand zur Verschlämmung bzw. nach anschließender Austrocknung zur Krustenbildung.

Böden mit hohen Tongehalten besitzen Nutzungseinschränkungen aufgrund geringer Wasserleitfähigkeiten und stellenweise hydromorpher Eigenschaften. Diese finden sich in Depressionen bzw. in den periodischen Überschwemmungsgebieten östlich und nördlich des Río Grande. BARBER et al. (1992) schätzen, dass die besseren Böden dieser Zone bei angepasstem Management und nur geringen Düngerapplikationen ca. 60 Jahre kontinuierlich bewirtschaftet werden könnten.

Im Chaco (Alluvialebene SE) dominieren schluffige Lehme (Abb.4). Diese sind gekennzeichnet durch mittlere Austauschkapazitäten, hohe Basensättigungen und schwach saure bis schwach alkalische Bodenreaktionen. Die Nährstoffversorgung mit N, P und K ist allgemein hoch, die Gehalte an organischer Substanz niedrig (Tab.2). Nutzungseinschränkungen liegen

\footnotetext{
5 Methode Olsen
} 
in dem mit zunehmender Breitenlage steigenden hydrologischen Defizit und dem häufigen Auftreten längerer Trockenperioden. Die Sandböden der Dünenbereiche (Arenales de Guanaco) sind wegen ihrer ungünstigen Nährstoffausstattung und dem schlechtem Wasserspeichervermögen nicht anbaugeeignet, werden aber für weidewirtschaftliche Zwecke genutzt. Die Bañados del Izozog im Südwesten bilden einen großen Überschwemmungskomplex, dessen tonige Böden hydromorphe Eigenschaften besitzen und aufgrund hoher Verdunstungsraten zu Versalzung und Alkalisierung neigen (AGRAR- \& HYDROTECHNIK 1973, 1974a, 1974b, GUAMAN 1990, KUHBACH 1997).

In der sanftwelligen Hügelzone des brasilianischen Schildes dominieren tiefgründig verwitterte Oxisole und Ultisole mit einem hohen Anteil an austauschbarem Fe- und AlOxiden, während in den Randbereichen, je nach Reliefposition, unterschiedlich mächtige Inceptisole vorherrschen. Bei den Bodenarten überwiegen lehmige Sande (Abb.4). Die Bodenreaktion liegt meist im schwach sauren Bereich. Die Böden sind basenreich und weisen niedrige Austauschkapazitäten auf. Die Nährstoffversorgung mit N, P und K ist niedrig bis mäßig, die Gehalte an organischer Substanz liegen auf mittlerem Niveau (Tab.2). Speziell die stark verwitterten Oxisole der Savannenbereiche besitzen eine geringe natürliche Fruchtbarkeit. Nutzungseinschränkungen bestehen hinsichtlich des sauren Charakters der Ausgangsgesteine, dem z.T. stark zerschnittenen Relief sowie im oft hohen Steingehalt des Oberbodens (BLASCHKE 1998, IPORRE 1996, KenNARD \& GHOLZ 2001, NAVARRO 1995).

\subsubsection{Natürliche Vegetation}

Im Osten von Bolivien ist durch das Zusammentreffen verschiedener biogeographischer Regionen eine kleinräumige Differenzierung unterschiedlicher Pflanzengesellschaften ausgeprägt. Das Spektrum reicht von halbimmergrünen Tieflandregenwäldern im humideren Norden, über laubabwerfende Trockenwälder, Baum- und Überschwemmungssavannen, bis zu den Dornbuschwäldern des Chaco. Die Ausbreitung der zonalen Waldgesellschaften wird von Savannen unterbrochen, deren Entstehung edaphisch bedingt ist. Diese sind hauptsächlich auf den nährstoffarmen, tiefgründig entwickelten Böden des brasilianischen Schildes (Oxisole) verbreitet. Im engeren Untersuchungsgebiet dominieren mesophytische und xerophytische Waldformationen des Cerrado und des Chaco, die mit zunehmender Breitenlage durch eine allmähliche Auflichtung des Kronendaches, geringere Wuchshöhen und stärkeren Laubabwurf in der Trockenzeit gekennzeichnet sind (BounOUA 2003). Die Artenzusammensetzung der Wälder variiert wiederum in Abhängigkeit edaphischer Faktoren. Auf sandigen Substraten finden sich in der Regel andere Vegetationstypen als auf tonigen. Ebenso bestimmt die Bodenfeuchte und demnach die räumliche und zeitliche Verteilung der Niederschläge den Laubabwurf der Bäume. In sehr feuchten Jahren ist dieser deutlich geringer (KILLEEN et al. 1998). Einflüsse aus dem amazonischen und subandinen Florenraum sind im Untersuchungsgebiet nur wenig verbreitet. 


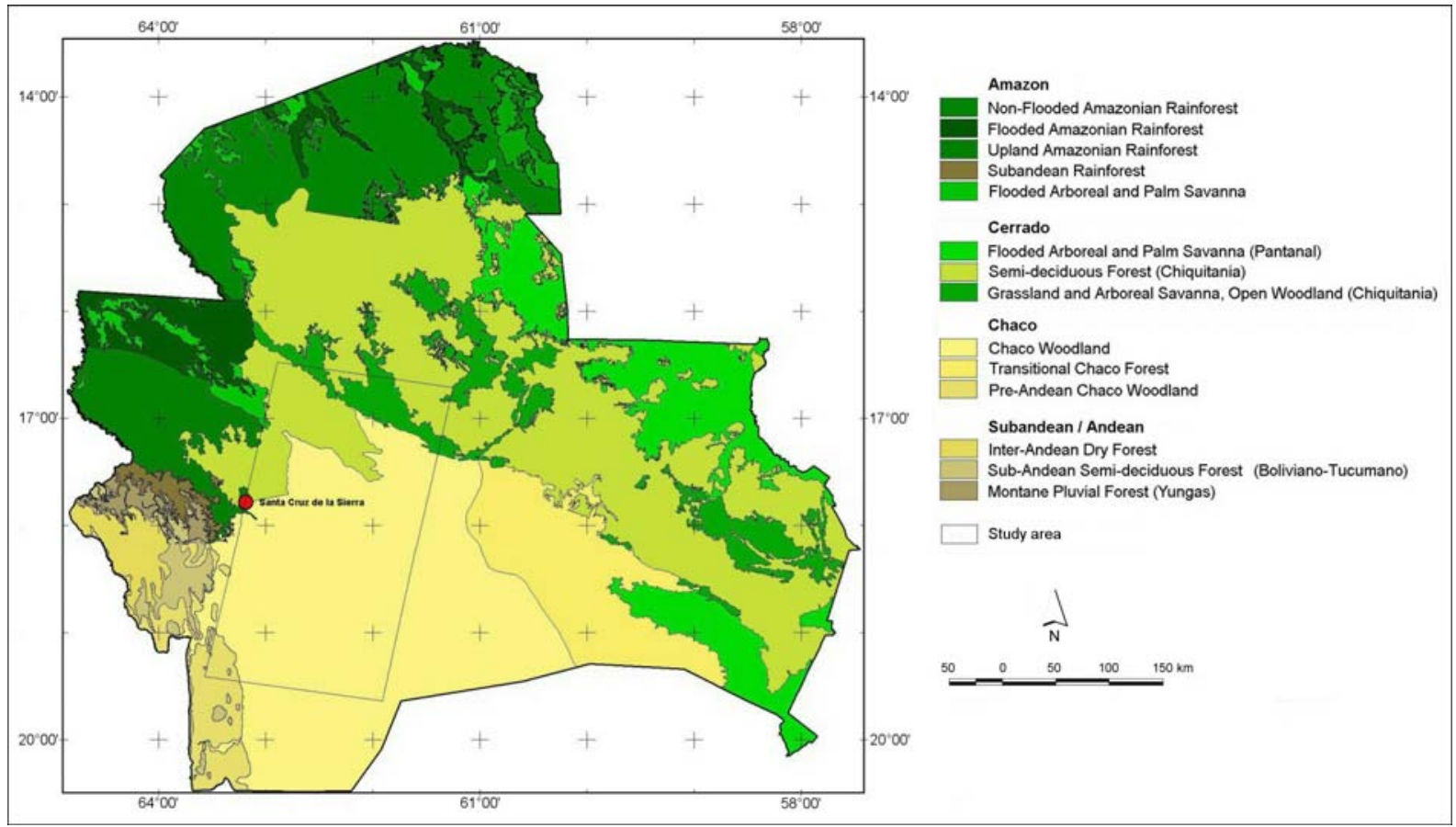

Abb. 5: Verbreitung der natürlichen Vegetation im Department Santa Cruz Quelle: IBISCH et al. (2004)

Die mesophytischen Trockenwälder besitzen zwei Baumschichten, die vereinzelt von Emergenten überragt werden (z.B. Schinopsis brasiliensis). Mittlere Bestandshöhen der obersten Baumschicht liegen bei 15-30 m mit einem, im Vergleich zu feuchttropischen Wäldern, offeneren Kronendach. Es handelt sich um voll- bzw. halblaubabwerfende Formationen (u.a. Anadenanthera colubrina, Acosmium cardenasii, Caesalpinia floribunda, Aspidosperma cylindrocarpon, Chorisia speciosa, Tabebuia impetiginosa). Der Unterwuchs ist gekennzeichnet durch einen hohen Anteil von Bäumen (zweite Baumschicht 3-15 m), Büschen, Kräutern und Lianen meist immergrüner Arten. Immergrüne Arten finden sich ebenfalls entlang von Gewässerläufen (Galeriewälder) verbreitet. Typische Baumart auf Böden hydromorpher Eigenschaften ist die Motacú Palme (Attalea phalerata). Dieser Trockenwaldtyp weist die höchste Diversitätsrate tropischer Trockenwälder in den Neotropen auf (KILlEEN et al. 1998, NAVARRO \& MALDONADO 2002).

Nach Süden bestimmen xerophytische Trockenwälder und Dornbuschformationen des Chaco das Landschaftsbild. Hauptvegetationsform ist ein niederwüchsiger, laubabwerfender Wald mit einem halboffenen Kronendach und mittleren Wuchshöhen zwischen 4-10 m (Emergenten bis $20 \mathrm{~m}$ ). Der Hauptteil der Biomasse wird von einer dichten, dornigen Strauchschicht gebildet, die bis $6 \mathrm{~m}$ Höhe erreichen kann. Typische Baumarten sind u.a. Aspidosperma quebracho-blanco, Schinopsis cornuta, Schinopsis lorentzii und die Baumkakteenart Browningia caineana sowie auf tonigen Substraten Tabebuia nodosa, Aspidosperma triternatum und die Palme Trithrinax schizophylla, in Bolivien unter dem Namen Saó bekannt. Eine Ausnahme in den Waldformationen des Chaco bilden die saisonal 
überschwemmten Wälder der Bañados del Izozog. Diese sind durch ein dichtes Kronendach und mittlere Wuchshöhen von 15-18 m gekennzeichnet. Charakteristische Baumart ist Albizia inundata. Der Unterwuchs wird durch eine zweite Baumschicht (8-10 m), eine Strauchschicht (2-4 m) und Lianen dominiert (NAVARRO \& MALDONADO 2002).

Nordwestlich von Santa Cruz sowie in Teilen des brasilianischen Schildes ist ein niederwüchsiger, sklerophyller Trockenwald mit durchschnittlichen Bestandshöhen von 5-10 m (u.a. Terminalia argentea, Curatella americana, Callisthene fasciculata) und einem Unterwuchs aus Büschen und Gräsern ausgebildet. Diese Formation leitet in die Savannenregionen (Baumsavannen, Grasländer) des Departments über, die sich sowohl auf geringmächtigen, skelettreichen Böden als auch tiefgründigen, nährstoffarmen Böden entwickelt haben. Beide Vegetationstypen werden von NAVARRO \& MALDONADO (2002) als Einheit betrachtet. In dieser Region befinden sich die Hauptweidezonen des Departments. Ein wichtiger Faktor für die Entwicklung der Savannen ist Feuer, dessen Einfluss auf das Ökosystem durch die Entwicklung widerstandsfähiger Pflanzen offensichtlich ist. Während die meisten Holzgewächse eine dicke, mehr oder weniger feuerresistente korkartige Schicht haben, überdauern die Fortpflanzungskeime von Gräsern und Büschen am Boden. Obwohl die Savannen für den Wiederwuchs frischer Gräser regelmäßig gebrannt werden, befinden sich die des brasilianischen Schildes zu großen Teilen noch in einem naturnahen Zustand (KILLEEN 1990).

\subsection{Landnutzung}

\subsubsection{Historische Entwicklung}

Die Entwicklung des landwirtschaftlichen Sektors in Ostbolivien setzte im wesentlichen mit der Agrarreform von 1953 ein. Vor dieser Zeit dienten landwirtschaftliche Tätigkeiten fast ausschließlich zur Subsistenz. Eine Anbindung des Tieflandes an überregionale Märkte war so gut wie nicht vorhanden bzw. wegen des unwegsamen Geländes und schlechter Straßen mit hohen Transportkosten verbunden (MONHEIM \& KÖSTER 1982). Mit dem Ausbau der Verkehrsinfrastruktur u.a. ins Hochland (Straße nach Cochabamba) bzw. mit dem Bau einer Bahnlinie nach Brasilien, wurde das östliche Tiefland ausgehend von der sogenannten zentralen Zone nördlich und östlich von Santa Cruz sukzessive erschlossen.

Als Folge der zunehmend weniger rentablen Minenwirtschaft in den Anden und einer damit verbundenen Zunahme des Bevölkerungsdrucks, wurde die ländliche Hochlandbevölkerung in den 1950er und 1960er Jahren sowohl über die Zuweisung von Land (zwischen 10-50 ha) als auch über günstige Kredite von staatlicher Seite ermutigt, sich im östlichen Tiefland niederzulassen. Die geplanten Kolonisationsprogramme waren Bestandteil eines staatlichen Entwicklungsprogramms, das über die Förderung des bolivianischen Agrarsektors das Ziel verfolgte, weniger vom Import landwirtschaftlicher Güter abhängig zu 
sein (PACHECO 1998, THIELE 1995). Durch die Schaffung geeigneter Verkehrsverbindungen zwischen Hoch- und Tiefland setzte eine sich beständig verstärkende Migration insbesondere in die nördlicheren Regenwaldgebiete ein, da dort wegen der höheren Feuchtigkeit weniger Missernten zu erwarten waren. Kamen Anfang der 1980er Jahre infolge einer bolivianischen Wirtschaftskrise alle staatlichen Programme zum Erliegen, hielt der Zuzug spontaner Kolonisten bis Mitte der 1990er Jahre stetig an. Zu diesem Zeitpunkt waren die Bodenpreise auf ein so hohes Niveau gestiegen, dass der Migrationsstrom zunehmend in die schnell wachsenden Außenbezirke von Santa Cruz ging (STEININGER et al. 2001). Lebten 1976 knapp 53 \% der Einwohner des Departments Santa Cruz in Städten, waren es 2001 bereits 76 \% (INE 2002).

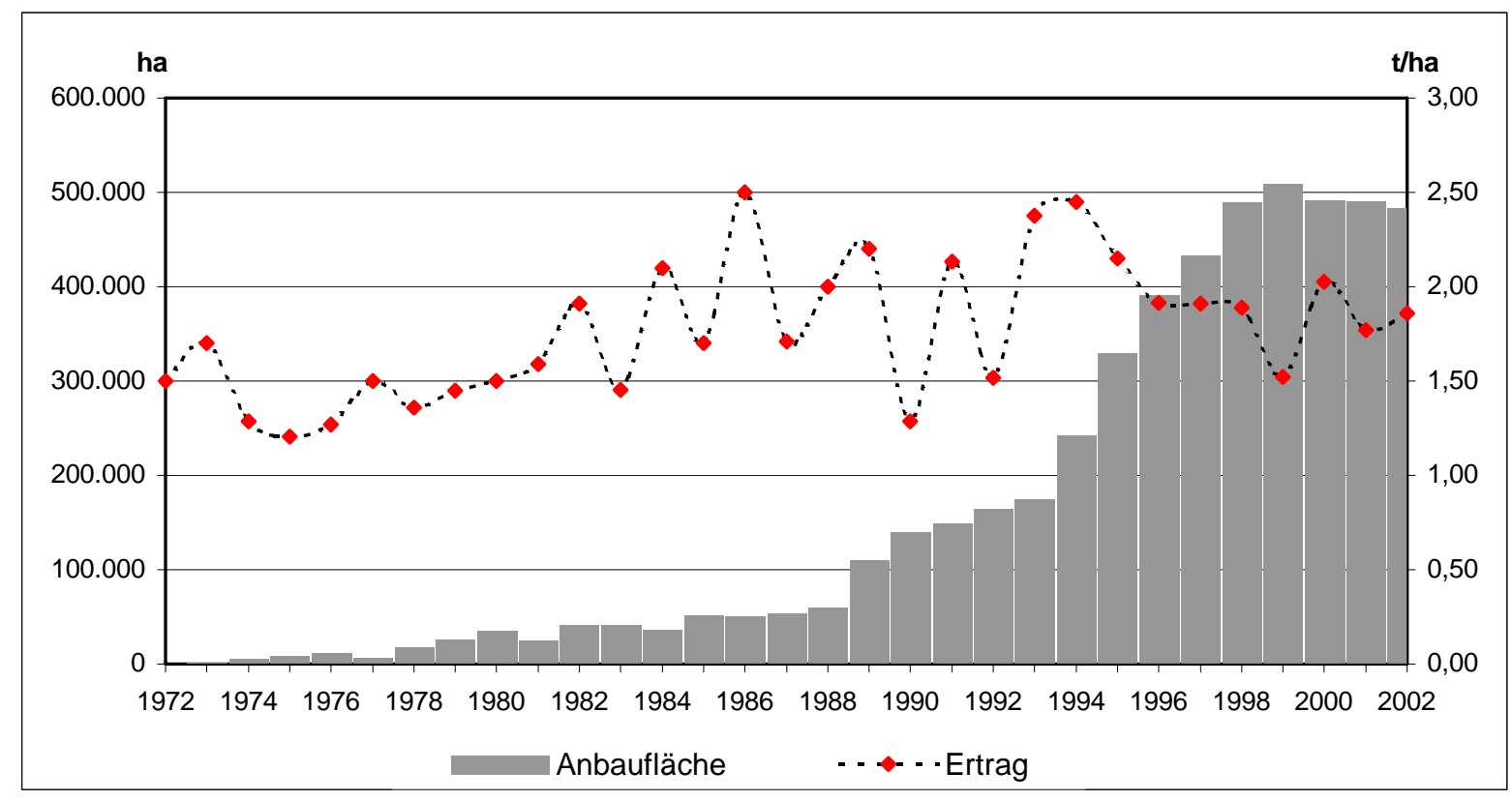

Abb. 6.1: Entwicklung des Sojabohnenanbaus im Department Santa Cruz von 1972 bis 2002 (Quelle: CAO 2002)

Die Entwicklung einer kommerziellen Landwirtschaft unter Einsatz moderner Technologie gelang ab Mitte der 1950er Jahre mit dem großflächigen Anbau von Zuckerrohr. Neben der kleinbäuerlichen Kolonisation entstand obendrein eine flächenintensive Agroindustrie, die sich zunächst aufgrund der besseren Absatzmöglichkeiten auf die zentrale Zone um Santa Cruz konzentrierte. Anfang der 1970er Jahre wurde Baumwolle wichtigstes Anbauprodukt. Sinkende Erträge und niedrige Weltmarktpreise waren Gründe, die jeweiligen Hauptanbauprodukte durch Produkte mit höherem Marktwert zu substituieren; 1950er Jahre Zuckerrohr $\rightarrow$ 1970er Jahre Baumwolle $\rightarrow$ 1990er Jahre Soja. Der Sojaanbau begann in den 1970er Jahren, erlangte aber erst Mitte der 1980er Jahre wirtschaftliche Bedeutung (THIELE 1995, URioste \& PACHECO 2001). Bis heute stellt Soja das mit Abstand wichtigste Anbauprodukt im Department Santa Cruz dar (Abb.6.1). 
Weiterer Bestandteil des landwirtschaftlichen Entwicklungsplans war die Anwerbung und Ansiedlung ausländischer Immigranten, vornehmlich Japaner und Mennoniten, deren Einwanderung gleichwohl durch den Staat gefördert wurde. Die drei großen japanischen Kolonien Okinawa 1 bis 3 wurden Anfang der 1960er Jahre nördlich von Santa Cruz gegründet, während die vor allem aus dem mexikanischen Hochland und dem Chaco Paraguays eingewanderten Mennoniten ihre Kolonien in trockeneren Gebieten östlich und südlich von Santa Cruz gründeten. Die Gründung neuerer Kolonien durch Mennoniten war, im Gegensatz zu denen der Kleinbauern, ein stetiger Prozess. Von 1954 bis 1968 wurde nahezu alle 3 bis 4 Jahre eine neue Kolonie gegründet. Mitte der 1980er Jahre wurden die ersten Mennonitenkolonien in der Expansionszone östlich des Río Grande gegründet, da viele Kolonien in der zentralen Zone die Grenzen ihres Landbesitzes erreicht hatten. Derzeit gibt es im Department Santa Cruz 39 Kolonien. Mennoniten leisten einen sehr hohen Beitrag zur bolivianischen Ökonomie. Allein ihr Beitrag zum bolivianischen Sojaexport betrug Mitte bis Ende der 1990er Jahre knapp 30 \% (CAO 2002, IADB 2001, KAIMOWITZ et al. 2001, MASRENA 2000).

Tab. 3: Anbauflächenentwicklung (in ha) der wichtigsten landwirtschaftlichen Produkte im Department Santa Cruz (1985-2002)

\begin{tabular}{ccccccccccc}
\hline Jahr & Reis & Mais & $\begin{array}{c}\text { Soja } \\
\text { (S) }\end{array}$ & $\begin{array}{c}\text { Soja } \\
(\mathbf{W})\end{array}$ & $\begin{array}{c}\text { Sonnen- } \\
\text { blume }\end{array}$ & $\begin{array}{c}\text { Zucker- } \\
\text { rohr }\end{array}$ & Weizen & $\begin{array}{c}\text { Baum- } \\
\text { wolle }\end{array}$ & $\begin{array}{c}\text { Sorghum Sorghum } \\
\text { (S) }\end{array}$ & $\begin{array}{c}\text { SW) } \\
\text { (W) }\end{array}$ \\
\hline 1985 & 80.000 & 70.000 & 51.000 & 12.000 & $*$ & 53.201 & 12.960 & 9.478 & $*$ & $*$ \\
1986 & 37.000 & 45.000 & 50.800 & 12.358 & $*$ & 53.869 & 10.000 & 10.831 & $*$ & $*$ \\
1987 & 50.000 & 32.000 & 53.878 & 12.500 & $*$ & 47.414 & 6.500 & 7.463 & $*$ & $*$ \\
1988 & 65.000 & 37.500 & 60.000 & 20.000 & $*$ & 40.520 & 4.000 & 9.710 & $*$ & $*$ \\
1989 & 58.500 & 35.000 & 110.000 & 30.000 & $*$ & 38.425 & 13.316 & 1.215 & $*$ & $*$ \\
1990 & 73.000 & 52.000 & 140.000 & 32.334 & 10.217 & 47.995 & 30.219 & 3.555 & $*$ & $*$ \\
1991 & 73.000 & 40.000 & 150.000 & 45.000 & 21.500 & 64.354 & 36.614 & 16.523 & 10.000 & 10.000 \\
1992 & 73.000 & 80.000 & 164.920 & 27.600 & 20.155 & 63.882 & 63.917 & 26.000 & 15.000 & 25.000 \\
1993 & 85.717 & 83.000 & 174.923 & 65.231 & 23.031 & 65.503 & 35.115 & 11.400 & 12.000 & 13.200 \\
1994 & 96.500 & 85.000 & 242.000 & 89.000 & 60.000 & 63.916 & 53.550 & 17.853 & 10.200 & 12.000 \\
1995 & 87.850 & 89.000 & 330.000 & 63.600 & 41.000 & 70.298 & 53.000 & 24.586 & 15.000 & 20.000 \\
1996 & 87.650 & 96.700 & 390.400 & 84.490 & 89.000 & 75.520 & 73.860 & 49.825 & 10.000 & 40.000 \\
1997 & 81.000 & 99.300 & 433.500 & 90.000 & 143.350 & 75.118 & 112.250 & 52.000 & 9.500 & 21.000 \\
1998 & 99.977 & 66.350 & 490.000 & 110.000 & 107.500 & 71.861 & 90.000 & 50.000 & 14.500 & 56.800 \\
1999 & 139.150 & 90.650 & 509.000 & 117.000 & 130.000 & 69.736 & 37.750 & 35.000 & 13.100 & 24.700 \\
2000 & 115.727 & 103.300 & 491.500 & 116.400 & 135.000 & 71.582 & 32.000 & 4.500 & 18.000 & 31.670 \\
2001 & 104.143 & 104.000 & 490.500 & 145.000 & 178.300 & 78.162 & 52.000 & 9.000 & 11.000 & 48.000 \\
2002 & 80.000 & 114.500 & 484.000 & 155.200 & 133.500 & 87.525 & 31.000 & 2.000 & 12.500 & 46.000 \\
\hline
\end{tabular}

S: Sommer, W: Winter (Quelle: CAO 2002)

Ende der 1980er Jahre wurde die Expansionszone östlich des Río Grande schrittweise durch agroindustrielle Großunternehmen erschlossen. Auslösend für die landwirtschaftliche Erschließung dieser Zone war ein in den 1980er Jahren über Kredite der Weltbank finanziertes Projekt (Proyecto Tierras Bajas del Este), das über Verbesserungen der Infrastrukur den Anbau erleichtern und ausbauen sollte (KAIMOwITZ et al. 1999). Der Landerwerb 
war günstig und über gleichzeitige niedrige Steuern sollten Anreize für Produzenten geschaffen werden, diese Zone in eine landwirtschaftliche Nutzung zu überführen. Primäres Ziel war, hauptsächlich über den Anbau von Soja, eine Ankurbelung der Exporte zu erreichen und damit eine langfristige ökonomische Sicherheit zu erzielen. Tatsächlich entwickelte sich Soja in den 1990er Jahren mitsamt seinen Derivaten zum Hauptexportprodukt und somit zu einem der wirtschaftlichen Motoren Boliviens. Die Exporterlöse lagen in dieser Zeit auf einem so hohen Niveau, dass Soja fast in Monokultur angebaut wurde und mehr die Quantität als die Qualität der Ernten zählte (URIOSTE \& PACHECO 2001, PACHECO 1998). Daher ist es nicht erstaunlich, dass mit dieser Periode die mit Abstand höchsten Entwaldungsraten im Department Santa Cruz einher gehen (Kap.4). Erst in jüngster Zeit beginnt aufgrund einer sich abzeichnenden Exportkrise dieses Sektors, infolge gesunkener Weltmarktpreise und abnehmender Erträge ein allmähliches Umdenken hin zu einer stärkeren Rotationswirtschaft mit Kulturen wie Sorghum, Sonnenblume, Mais und Baumwolle sowie der Einführung von Brachezeiten.

Zusammenfassend lassen sich mit Beginn der 1950er Jahre drei Phasen der landwirtschaftlichen Entwicklung im Department Santa Cruz konstatieren: Die erste zielte auf eine weitgehende Unabhängigkeit gegenüber Importen, während die zweite mittels struktureller Anpassungen (z.B. stärkere Diversifikation der Anbauprodukte) der Konsolidierung des erworbenen Status Quo diente. Die letzte, aktuelle Phase zeigt eine deutliche Ausrichtung hin $\mathrm{zu}$ einer exportorientierten Landwirtschaft (PACHECO 2001, 1998, IADB 2000). Insgesamt hat sich diese Zone in wirtschaftlicher als auch demographischer Hinsicht in den letzten 50 Jahren zur dynamischsten in Bolivien entwickelt (IADB 2000).

\subsubsection{Landnutzungssysteme und aktuelle Landnutzung}

Im Untersuchungsgebiet lassen sich verschiedene landwirtschaftliche Produzenten hinsichtlich ihres angewandten Landnutzungssystems und des Mechanisierungsgrads unterscheiden:

- Kleinbauern, häufig als spontane Siedler entlang von Straßen angesiedelt oder in nationalen Kolonien organisiert, deren Besitzgrößen weniger als 50 ha betragen. Der Anbau erfolg bei den Kleinbauern mit der geringsten Kapitalausstattung in Subsistenzwirtschaft oder orientiert sich wegen der hohen Transportkosten ausschließlich an den nächstgelegenen Märkten (PACHECO 1998). Betrieben wird das traditionelle System des Brandrodungsfeldbaus (slash and burn). Dabei handelt es sich um eine manuelle Form der Bewirtschaftung, wobei Felder nach dem Brennen einer Parzelle wenige Jahre, zumeist in Rotation mit anderen Kulturen, bewirtschaftet werden. Eine maschinelle Bearbeitung der 
Felder ist nicht möglich, da die nur zum Teil verbrannten Baumstümpfe nicht entfernt werden. Sinken die Erträge infolge der Zunahme von Unkräutern, schließt sich eine mehrjährige Brache an, bevor die Felder erneut gebrannt und genutzt werden (CIAT 1980, Gaugin et al. 2002, Kennard 2002, Thiele 1993a, Weisbach et al. 2002). Das System zeichnet sich aufgrund der geringen Kapitalausstattung durch einen hohen Bracheanteil aus. Hauptanbauprodukte sind Mais, Reis, Yuca, Bohne, Süßkartoffel und als Dauerkultur Banane. Häufig werden einige Rinder und Kleinvieh (Schweine und Hühner) für den Eigenbedarf gehalten. Eine Düngung erfolgt lediglich über den Verbleib von Pflanzenresten auf den Feldern oder über den Einsatz von Tierdung. Kunstdünger werden nicht eingesetzt (IADB 2000).

Die Kleinbauern der nationalen Kolonien betreiben bereits zu einem gewissen Grad mechanisierten Anbau erreicht, so dass ihr Spektrum von der traditionellen Nutzung bis hin zur Bodenabearbeitung unter Einsatz von Maschinen reicht. Die Bearbeitung erfolgt semi-mechanisiert (einige Anbauflächen werden maschinell, andere manuell bearbeitet) bzw. vollmechanisiert (alle Anbautätigkeiten werden mit maschineller Unterstützung durchgeführt). Die Maschinen werden in der Regel geliehen (URIOSTE \& PACHECO 2001). Die Anbauprodukte orientieren sich am nationalen Markt. Mit steigendem Mechanisierungsgrad gewinnen Kulturen wie Baumwolle, Zuckerrohr und Sonnenblume an Bedeutung. Das durch den höheren Mechanisierungsgrad gekennzeichnete Betriebssystem zeigt gegenüber dem traditionellen System, durch Anpassung der Fruchtfolgen an die jeweilige Marktlage, eine geringere Rotation der Kulturen sowie einen höheren Nutzflächenanteil bei gleichzeitig weniger Bracheflächen. Ernterückstände werden auf dem Feld belassen und bei erneuter Bestellung untergepflügt. Kunstdünger werden nicht appliziert, aber Pestizide zur Schädlingsbekämpfung. Viehhaltung erfolgt oft in Wechselwirtschaft mit Kulturen (IADB 2000). Im Vergleich aller Produzenten lässt sich bei den Kleinbauern der höchste Diversifizierungsgrad in den Anbauprodukten erkennen; dieser wiederum sinkt mit steigendem Mechanisierungsgrad.

- Produzenten ausländischer Kolonien (Mennoniten, Japaner) mit variierenden Besitzgrößen von durchschnittlich 50 bis 1.000 ha, die vollmechanisierten Anbau betreiben. Hauptanbauprodukte sind Soja, Sonnenblume und Weizen sowie in geringerem Umfang Reis (Japaner), Mais und Sorghum (Mennoniten). Sorghum und Mais werden bei den Mennoniten als Futterpflanze eingesetzt (Tab.3). Eine Düngung der Felder erfolgt nicht, Pestizide aber werden appliziert (URIOSTE \& PACHECO 2001). Betrieben wird eine permanente Rotation der Kulturen mit in der Regel Soja als Sommerkultur. Ebenso spielt Viehhaltung eine größere Rolle. Bei den Mennoniten werden knapp 20-30 \% der jedem Siedler zur Verfügung stehenden Nutzfläche für Weidezwecke genutzt (IADB 2000). Neben der 
Fleischproduktion für den Eigenbedarf wird in größerem Umfang Milchviehhaltung und Veredelungswirtschaft (Käse) betrieben. Das mennonitische Betriebssystem kennt keine Brachezeiten, d.h. alle Felder stehen seit ihrer Rodung unter kontinuierlicher Nutzung. Mennoniten und Japaner haben durch die komplette Rodung ihrer Landbesitze große, zusammenhängende Flächen von Kulturland geschaffen (Abb.6.2).

- Agroindustrielle Betriebe mit Betriebsgrößen von mehr als 2.000 ha mehrheitlich brasilianischer Eigner und mittelgroße Betriebe nationaler Produzenten mit Betriebsgrößen unter 2.000 ha. Typisches Erscheinungsbild sind kilometerlange Felder, die von 20-40 m breiten Windschutzstreifen des ursprünglichen Waldes eingefasst sind (Abb.6.2). Die vollmechanisierte Produktion ist auf wenige, exportorientierte Produkte konzentriert und versorgt zudem den nationalen Markt mit Agrargütern. Investitionen werden größtenteils über Bankkredite getätigt. Hauptanbauprodukt ist Soja (Sommer), das mit Sonnenblume, Weizen oder Sorghum im Winter in Rotation gepflanzt wird. Daneben wird Baumwolle und Mais sowie Soja auch im Winter angebaut (Tab.3). Düngergaben können erfolgen, sind aber wegen der Profitorientiertheit und den damit verbundenen zusätzlichen Kosten nicht üblich. Pestizide werden appliziert. Verschiedentlich kommen im Süden der Expansionszone große Bewässerungssysteme (Center-Pivot Anlagen) zum Einsatz (IADB 2000, KAIMOWITZ et al. 1999, PACHECO 1998).

- Extensive Weidewirtschaft mit durchschnittlichen Betriebsgrößen von 2.500 bis 20.000 ha in den natürlichen Graslandschaften des Chaco und des brasilianischen Schildes (Chiquitania). In den natürlichen Weideregionen determiniert sich die Viehhaltung über die Verfügbarkeit von Wasser und natürlicher Futterpflanzen, wonach die sehr großen Weideflächen im Chaco die geringste Bestockungsdichte pro Hektar aufweisen. Mit einem Viehbesatzanteil von $44 \%$ (CAO 2002) und einem Weideflächenanteil von $45 \%$ (FEGASACRUZ 1999) ist die Chiquitania die in dieser Hinsicht wichtigste Zone im Department, dies nicht zuletzt durch die ergänzende Kultivierung hochwertiger Weidegräser, wie z.B. Panicum maximum, Brachiaria decumbens und Brachiaria brizantha (KILLEEN et. al 1990). Intensive Viehwirtschaft mit hoher Bestockungsdichte aber geringem Flächenanteil wird auf den künstlichen Weiden der zentralen Zone nördlich und südlich von Santa Cruz sowie zu einem geringen Teil in der Expansionszone betrieben.

Die Viehwirtschaft dient überwiegend der Fleischproduktion für den lokalen und regionalen Bedarf. Im Vergleich mit den großen Produzenten Brasiliens, Argentiniens und Paraguays sind die bolivianischen Viehwirte nicht konkurrenzfähig. Ein geringer Teil wird auf den Markt der Andengemeinschaft (Comunidad Andina de Naciones, CAN) exportiert. Auf den Weideflächen im Großraum Santa Cruz wird zudem in kleinerem Umfang Milchviehhaltung betrieben (IADB 2000, URIOSTE \& PACHECO 2001). 


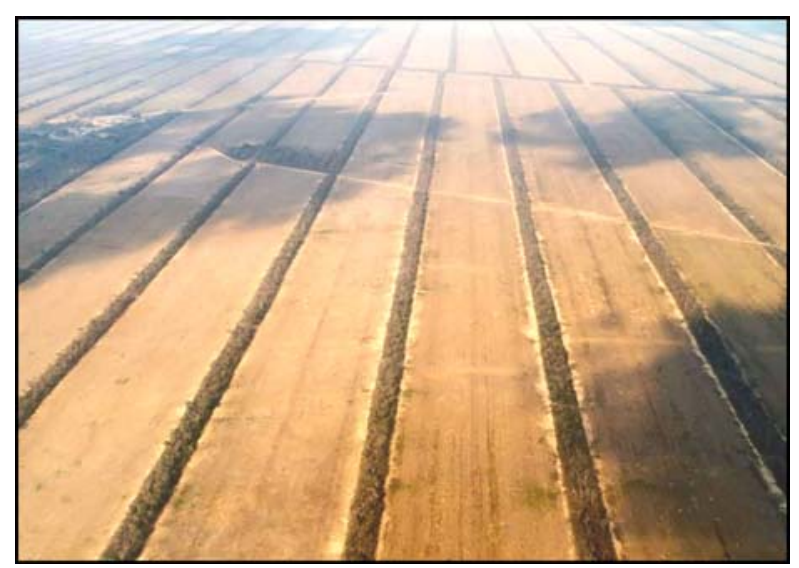

1.

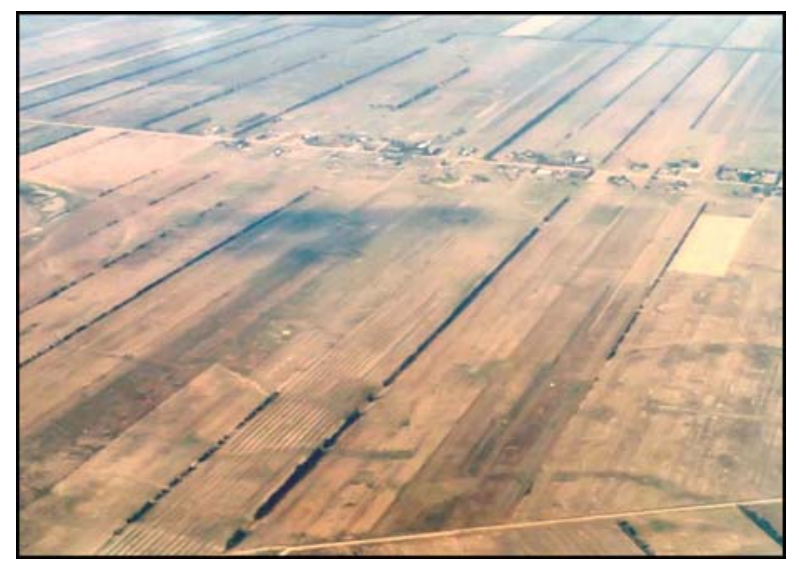

3.

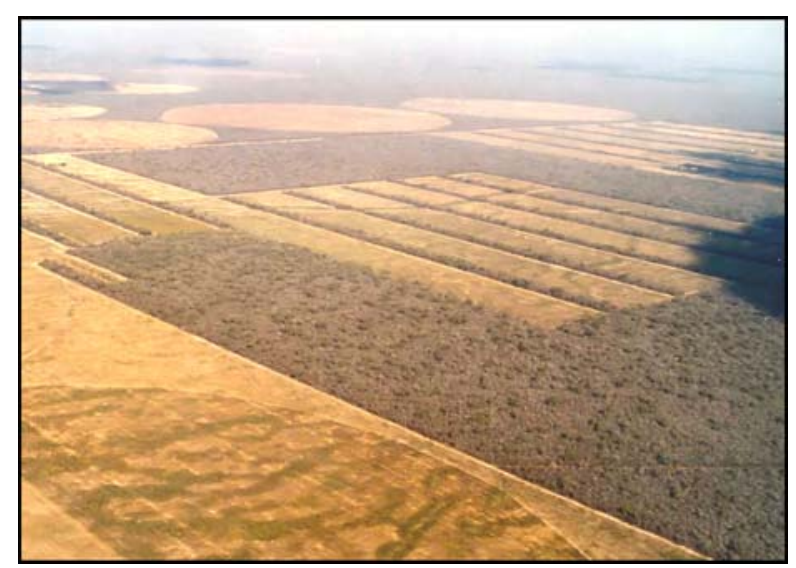

2 .

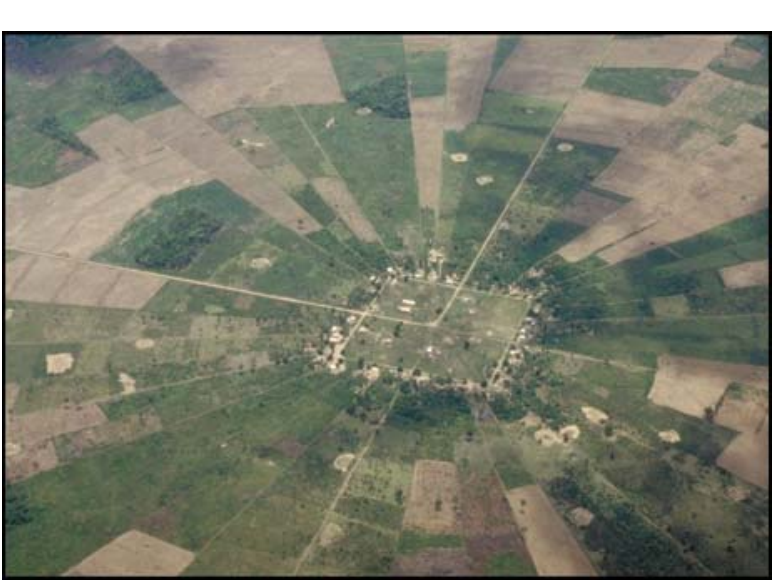

4.

(Quelle: http://earthobservatory.nasa.gov/)

Abb. 6: Luftbildaufnahmen landwirtschaftlicher Betriebssysteme im Department Santa Cruz

1. Agroindustrie 2. Agroindustrie mit Pivot-Bewässerung (Bildhintergrund) 3. Mennoniten 4. Kleinbauern

Grundsätzlich lassen sich die unterschiedlichen Landnutzungssysteme grob regional differenzieren: Kleinbauern (nationale Kolonien, spontane Siedler) haben sich vornehmlich im feuchteren Nordwesten angesiedelt. Vollmechanisierte, großflächige Landwirtschaft wird auf den fruchtbaren Alluvialböden östlich (Expansionszone) und westlich (zentrale Zone) des Río Grande bis etwa $18^{\circ}$ südl. Breite betrieben. Die Viehwirtschaft konzentriert sich auf die Naturweiden des brasilianischen Schildes im Nordosten und Osten sowie des Chaco im Süden. Hauptexportprodukte sind Soja und Sonnenblume bzw. deren Derivate (Ö1, Mehl, Kerne) sowie Baumwolle als Halbfertig- und Fertigprodukt. Getreide wie Mais, Sorghum und Weizen sind vornehmlich am Binnenmarkt orientiert. In den Hauptanbauzonen werden zwei Anbauzyklen pro Jahr durchgeführt, mehrheitlich mit Soja als Sommer- und Winterkultur (Tab.3). Eine Düngung der Felder unterbleibt in der Regel bei allen Landnutzungssystemen wegen der damit verbundenen hohen Kosten ${ }^{6}$. Der traditionelle Brandrodungsfeldbau verliert in der Region in den letzten Jahren zunehmend an Bedeutung, da die in den Kolonien organisierten Kleinbauern vermehrt auf mechanisierte Bewirtschaftungsformen umsteigen (MARTÍNEZ 2004). Die Viehwirtschaft dient fast ausschließlich der Fleisch- und nur zu einem

\footnotetext{
${ }^{6}$ Bei den Mennoniten werden hierfür oft religiöse Gründe genannt, die jedoch nicht näher spezifiziert werden.
} 
geringen Teil der Milchproduktion. Einen Überblick über die aktuelle Verteilung der Landnutzung im Department Santa Cruz gibt Tabelle 4.

Tab. 4: Verteilung der Landnutzung im Department Santa Cruz

\begin{tabular}{lrr}
\hline Landnutzung & $\begin{array}{r}\text { Fläche } \\
\left(\mathrm{km}^{2}\right)\end{array}$ & $(\%)$ \\
\hline Ackerbau & 28.536 & 7,7 \\
Agrosilvopastorale Systeme & 10.573 & 2,9 \\
Wälder (z.T. mit Waldweidewirtschaft) & 117.185 & 31,6 \\
Weidewirtschaft - extensiv & 65.072 & 17,6 \\
Weidewirtschaft - intensiv & 4.006 & 1,1 \\
Schutzgebiete (z.T. mit Weidewirtschaft) & 39.725 & 10,7 \\
Schutzgebiete & 93.581 & 25,2 \\
andere & 11.942 & 3,2 \\
\hline Total & 370.620 & 100,0 \\
\hline & Quelle: (CAO 2002, verändert)
\end{tabular}




\section{Datengrundlage Und Methodik}

Die integrative Analyse und Bewertung von Prozessen der Bodendegradation setzt bei dem hier vorgestellten Verfahren eine Vielzahl an Eingangsinformationen voraus. Diese wurden im Sinne der Zielsetzung als Flächendatensätze (Landnutzung, Boden, Relief, Klima) aufbereitet und räumlich analysiert. Anhand von Satellitendaten wurde sowohl die Entwicklung der Landnutzung ermittelt, als auch die regionale Verteilung der Landnutzungssysteme abgeleitet (Kap.4). Gemäß dem für die Regionalisierung von Bodeneigenschaften gewählten Ansatz von BÖHNER \& KÖTHE (2003) wurden primäre und sekundäre Reliefparameter auf Grundlage eines digitalen Geländemodells (DGM) berechnet sowie Klimaparameter über ein von BÖHNER (2004) entwickeltes semi-empirisches Regionalisierungskonzept unter Nutzung von Daten eines allgemeinen Zirkulationsmodells (GCM) und lokaler Klimastationsdaten räumlich generiert (Kap.3.4).

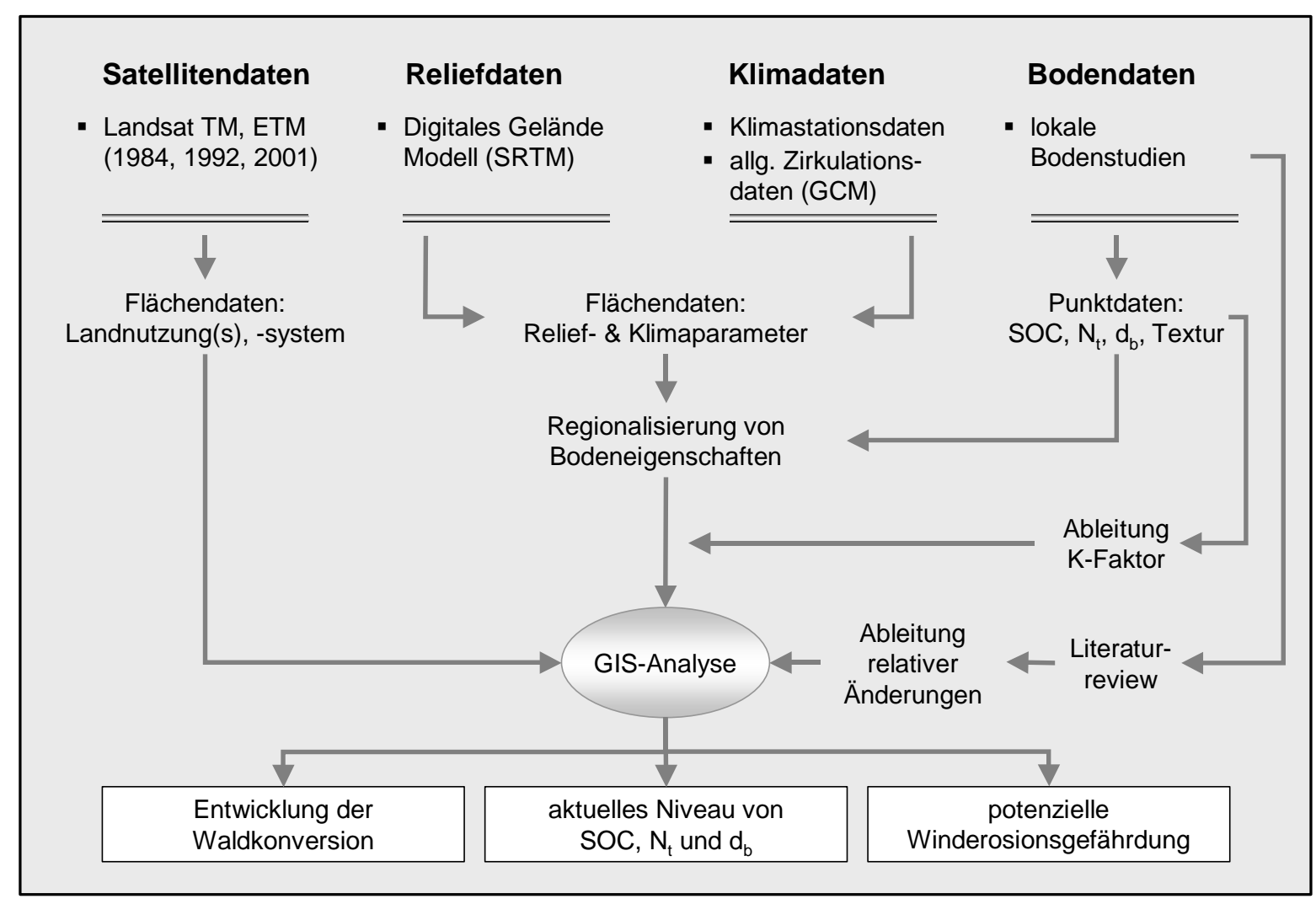

Abb. 7: Schema der Vorgehensweise

Bodenparameter wurden aus vorhandenen Profilbeschreibungen abgeleitet, homogenisiert und in einer Datenbank zusammengestellt (Kap.3.2). Über schrittweise multiple Regression punktuell erhobener Bodeneigenschaften ohne Beeinflussung durch agrarische Nutzung mit Klima- und Reliefparametern wurden Abhängigkeiten überprüft und mittels geostatistischer Interpolation regionalisiert (Kap.5.1.1). Aus lokalen Bodenuntersuchungen und Literatur- 
angaben wurden Trends potenzieller Änderungen chemischer und physikalischer Eigenschaften des Bodens infolge landwirtschaftlicher Nutzung identifiziert und als metrische Prognosevariablen in die Fläche übertragen. Zusätzlich wurde über die Bestimmung der Häufigkeit erosiver Winde und die relative Erodibilität der Böden (K-Faktor), die Bodenerosionsgefährdung durch Wind analysiert (Kap.5.1.4).

Nachfolgend werden die in der Studie verwendeten Daten und deren Aufbereitung im Sinne der Zielsetzung beschrieben. Eine Übersicht über das methodische Konzept ist in Abb.7 dargestellt.

\subsection{Satellitendaten}

Der Studie lagen die in Tab.5 zusammengestellten Satellitendaten der Fernerkundungssysteme Landsat-TM und Landsat-ETM+ zugrunde. Für jedes Jahr standen zwei systemkorrigierte WRS-2 Szenen der path/row Bezeichnungen 230/72 und 230/73 zur Verfügung (Abb.8). Alle Szenen waren von Seiten des Distributors radiometrisch vorverarbeitet und auf die UTM-Projektion, Zone 20 S (WGS 84) georeferenziert. Alle Aufnahmezeitpunkte liegen in der Trockenzeit. Mit Ausnahme geringer Bewölkung $(<1 \%)$ der 230/73er Szene des Jahres 1992 waren alle Aufnahmen wolkenfrei. Für weitere technische Daten der verwendeten Satellitenbilder sei auf Tabelle A2 im Anhang verwiesen. Als ergänzende Informationsquellen wurden alle im Kartenverzeichnis aufgelisteten topographischen und thematischen Karten verwendet.

Tab. 5: Verwendete Satellitenbildszenen

\begin{tabular}{lcc}
\hline Sensor & Aufnahmedatum & WRS (path/row) \\
\hline Landsat 5 TM & 30.09 .1984 & $230 / 72$ \\
Landsat 5 TM & 30.09 .1984 & $230 / 73$ \\
Landsat 4 TM & 10.07 .1992 & $230 / 72$ \\
Landsat 4 TM & 10.07 .1992 & $230 / 73$ \\
Landsat 7 ETM+ & 04.08 .2001 & $230 / 72$ \\
Landsat 7 ETM+ & 04.08 .2001 & $230 / 73$ \\
\hline
\end{tabular}

Das Untersuchungsgebiet wurde während eines insgesamt viermonatigen Geländeaufenthaltes von Anfang Juli bis Ende Oktober 2002 intensiv begutachtet und mittels GPS Referenzpunkte für die anschließende Satellitenbildauswertung eingemessen. Ergänzend wurde im September 2002 eine Überfliegung durchgeführt. Die Geländebeobachtungen dienten als Interpretationsgrundlage der verschiedenen Landnutzungssysteme ebenso wie für die Beurteilung der Reflexionsklassen im Satellitenbild. 


\section{Geometrische Entzerrung}

Um die Aufnahmen der unterschiedlichen Zeitpunkte räumlich in Beziehung zu setzen, wurden diese in eine einheitliche Geometrie gebracht. Zu diesem Zweck wurden zunächst die im Gelände mittels GPS aufgenommenen Kontrollpunkte den Szenen überlagert und visuell überprüft. Die höchste Lagegenauigkeit mit maximalen Abweichungen unterhalb eines Pixels $(30 \mathrm{~m})$ boten die Landsat 7 Szenen, die für die weitere Verarbeitung als Referenzszenen herangezogen wurden. Alle anderen Szenen wurden über eine Bild-zu-Bild Entzerrung auf Grundlage der Referenzszenen co-registriert und im Falle der 1992er Szenen, deren ursprüngliche räumliche Auflösungen bei $28,5 \mathrm{~m}$ lagen, auf $30 \mathrm{~m}$ resampelt ${ }^{7}$. Als Interpolationsmethode wurde Nearest-Neighbor gewählt, da bei diesem Verfahren die spektrale Information der Aufnahmen nicht verändert wird (LILLESAND \& KIEFER 2000).

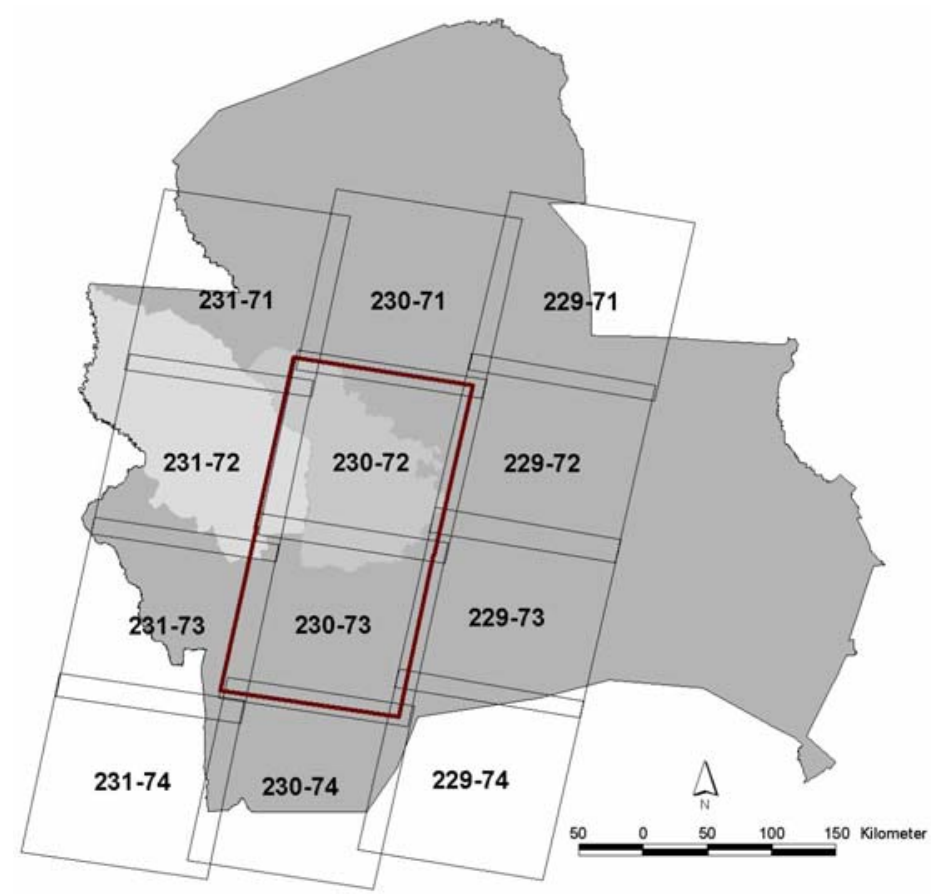

Abb. 8: Räumliche Anordnung der Landsat Szenen (WRS Kombination) im Department Santa Cruz

Anm.: Die 230-72 und 230-73 decken das engere Untersuchungsgebiet ab (die beiden in den helleren Grautönen hervorgehobenen Gebiete umfassen die sogenannte zentrale Zone und Expansionszone

\section{Untersuchungsgebiet}

Die Aufnahmestreifen (path) der Landsat-Szenen decken im Vergleich der Aufnahmezeitpunkte nicht exakt die gleiche Gebietsfläche ab. Von 1984 nach 2001 zeigt sich einen leichter Versatz nach Osten. Für die Zugrundelegung einer einheitlichen Bezugsfläche wurden

\footnotetext{
7 Die Bildverarbeitung erfolgte mit der Software ENVI Ver. 3.6 (C Research Systems Inc.). GIS-Operationen wurden mit der Software ArcView 3.2, ArcGIS 8.3 (C Environmental Systems Research Institute, Inc.) und SAGA GIS (System for Automated Geoscientific Analyses) durchgeführt. SAGA GIS ist ein nicht-kommerzielles, modular aufgebautes GIS, entwickelt von der Arbeitsgruppe Geosystemanalyse des Geographischen Instituts der Universität Göttingen (http://www.saga-gis.uni-goettingen.de/html/index.php).
} 
die Überschneidungsbereiche aller Szenen ausmaskiert und die daraus resultierende Fläche als Untersuchungsgebiet definiert. Daraus errechnet sich eine Gebietsgröße von $58.197 \mathrm{~km}^{2}$, entsprechend $16 \%$ der Departmentfläche.

Die Analyse der Satellitendaten und die daraus erfolgte Ableitung von Informationen über die Entwicklung der Landnutzung, den Rückgang der natürlichen Vegetation (Waldkonversion) und die Verteilung der Landnutzungssysteme im Untersuchungsgebiet werden detailliert in Kapitel 4 beschrieben.

\subsection{Ableitung von Bodendaten}

Für die Interpretation von Bodeneigenschaften und deren Regionalisierung wurden Bodendaten aus insgesamt 370 Profilbeschreibungen abgeleitet und in einer Datenbank zusammengestellt. Die Daten entstammen zahlreichen, in den letzten 30 Jahren im Raum Santa Cruz durchgeführten Arbeiten (Tab.6). Die ältesten Analysen datieren aus den Jahren 1973 und 1974 und gehen auf ein deutsch-bolivianisches Gemeinschaftsprojekt zurück, das die Evaluation des landwirtschaftlichen Potenzials der Chacoregion zum Gegenstand hatte (AgraR- \& HydroteCHNIK 1973, 1974a, 1974b). Ein Großteil der Daten wurden vom Centro de Investigacion Agrícola Tropical (CIAT) in Santa Cruz erhoben, das sich seit Anfang der 1980er Jahre mit potenziellen Auswirkungen verschiedener Bewirtschaftungsweisen auf den Boden im Raum Santa Cruz beschäftigt. Konzentrierten sich die Arbeiten von CIAT anfangs noch auf die Kolonisationsgebiete der Kleinbauern und den mechanisierten Anbau in der zentralen Zone, verlagerte sich der Schwerpunkt später gänzlich auf mechanisierte Systeme, da diese in wesentlich größerem Umfang zur Bodendegradation beitragen. In enger Verbindung mit den Arbeiten des CIAT stehen die der Agrarfakultät der Universidad Gabriel René Moreno in Santa Cruz. Umfangreiche Bodendaten gehen auf Untersuchungen von GuAman (1988a, 1988b, 1999) zurück, die im Rahmen staatlicher Projekte zur Erfassung der natürlichen Ressourcen im Raum Santa Cruz erhoben wurden. Seit Mitte der 1980er Jahre wurden von GEROLD mehrere Arbeiten zur Bodennutzungsproblematik in Ostbolivien durchgeführt, die in verschiedenen Abschlussarbeiten der Universität Göttingen ihre Fortsetzung fanden. Ergänzend wurden Profildaten der im Internet verfügbaren Datenbank SOTERLAC 1.0 (Soil and Terrain Database for Latin America) ${ }^{8}$ hinzugezogen (FAO 1998).

Umfangreiche Informationen über die Böden des Projektgebietes gibt zudem der für das Department Santa Cruz erstellte Landnutzungsplan PLUS (CORDECRUZ et al. 1996), dessen Grundlage diverse, zu unterschiedlichen Zeitpunkten durchgeführte Bodenuntersuchungen bilden. Leider existiert aus diesem umfassenden Projekt keine metrische Bodendatenbank, auf die hätte zurückgegriffen werden können. Hauptprodukte des PLUS sind analoge Karten im

\footnotetext{
${ }^{8}$ http://grid.cr.usgs.gov/unepdownload/form.php?type=soterlac
} 
Maßstab 1 : 500.000, denen allgemeine, nicht metrische Informationen der Böden und deren Nutzungspotenzial sowie Angaben zur Vegetation entnommen werden können.

Tab. 6: Verwendete Profilbeschreibungen der Bodendatenbank

\begin{tabular}{|c|c|c|c|}
\hline Quelle & Aufnahmedatum & Region & Anzahl Profile \\
\hline AGRAR- \& HYDROTECHNIK (1973) & 1973 & Alluvialebene SE & 8 \\
\hline AGRAR- \& HYDROTECHNIK (1974) & 1973 & Alluvialebene SE & 16 \\
\hline AGRAR- \& HYDROTECHNIK (1974) & 1973 & Alluvialebene SE & 14 \\
\hline \multirow[t]{2}{*}{ KIDMAN et al. (1980) } & 1979 & Alluvialebene NE & 15 \\
\hline & & Alluvialebene NW & 27 \\
\hline \multirow[t]{2}{*}{ GEROLD (1986) } & 1982 & Alluvialebene NW & 7 \\
\hline & & Subandin (Andenfußzone) & 4 \\
\hline \multirow[t]{2}{*}{ MONTENEGRO (1987), Ruíz (1987) } & $1978-1986$ & Alluvialebene NW & 14 \\
\hline & & Subandin (Andenfußzone) & 4 \\
\hline DIAZ \& BARBER (1988) & 1984-1985 & Alluvialebene NW & 2 \\
\hline GUAMAN (1988) & 1984-1988 & Alluvialebene NE & 27 \\
\hline GUAMAN (1988) & 1987 & Alluvialebene NE & 36 \\
\hline BARBER et al. (1989) & 1989 & Alluvialebene NW & 13 \\
\hline \multirow[t]{3}{*}{ BARBER (1989) } & 1988 & Alluvialebene NW & 36 \\
\hline & & Alluvialebene SW & 11 \\
\hline & & Subandin (Andenfußzone) & 2 \\
\hline \multirow[t]{2}{*}{ Ruiz et al. (1990) } & $1987-1988$ & Alluvialebene NW & 7 \\
\hline & & Alluvialebene SW & 4 \\
\hline BARBER \& NAVARRO (1992) & 1990 & Alluvialebene SW & 2 \\
\hline BARBOSA et al. (1989), BARBER (1994) & 1985 & Alluvialebene NW & 1 \\
\hline BARBER \& DÍAZ (1994), BARBER (1994b) & 1986 & Alluvialebene NW & 1 \\
\hline BARBER \& ROMERO (1994) & 1991 & Alluvialebene NE & 6 \\
\hline BARBER \& NAVARRO (1994) & 1989 & Alluvialebene SW & 3 \\
\hline BARBER et al. (1996) & 1989 & Alluvialebene NW & 2 \\
\hline IPORRE BELLIDO (1996) & 1995 & brasilianischer Schild & 30 \\
\hline KUHBACH (1997) & 1997 & Alluvialebene SE & 15 \\
\hline \multirow[t]{4}{*}{ FAO (1998) - SOTER } & $1978-1988$ & Alluvialebene NE & 1 \\
\hline & & brasilianischer Schild & 6 \\
\hline & & Alluvialebene SE & 1 \\
\hline & & Subandin (Andenfußzone) & 1 \\
\hline BLASCHKE (1998) & 1996 & brasilianischer Schild & 12 \\
\hline \multirow[t]{2}{*}{ GUAMAN (1999) } & 1979-1999 & Alluvialebene SW & 19 \\
\hline & & Subandin (Andenfußzone) & 11 \\
\hline KARSTEN (2000) & 1998 & Alluvialebene SW & 6 \\
\hline MARKUSSEN (2000) & 1998 & Alluvialebene SW & 6 \\
\hline$\Sigma$ & & & 370 \\
\hline
\end{tabular}

(NW: Nordwesten, NE: Nordosten, SW: Südwesten, SE: Südosten)

Ein grundsätzliches Problem bei der Verwendung von Bodendaten aus verschiedenen Quellen besteht in deren heterogener Qualität. Das setzt voraus, dass die Daten zunächst bestmöglich aufeinander abgestimmt werden. Je nach Art der Studie wurde die Datenerhebung (bspw. laboranalytische Verfahren) unterschiedlich genau beschrieben und war nicht in allen Fällen en detail nachvollziehbar, folglich verbleibt eine gewisse Unsicherheit bei der Vergleichbarkeit. Abweichende Größenordnungen der Variablenwerte sind möglich, 
beziehen sich die Angaben auf unterschiedliche Entnahmetiefen. Diese Diskrepanzen mussten toleriert werden, um eine für die Gebietsgröße ausreichende Quantität an Bodendaten zu erhalten. Werte aus nicht vergleichbaren Verfahren wurden eliminiert. Die Stoffgehalte wurden auf ihre Plausibilität geprüft und fragwürdige Werte als Ausreißer aus dem Datenbestand entfernt. Als Orientierungshilfe dienten dabei die Richtlinien zur Bewertung bodenchemischer Eigenschaften von CIAT (Tab.A1, Anhang).

Alle abgeleiteten Angaben beziehen sich auf den Oberboden. In dieser Zone treten die deutlichsten Veränderungen von Bodeneigenschaften durch landwirtschaftliche Einwirkungen auf (BATJEs \& DiJKSHOORN 1999, KOZLOWSKi 1999, VAN LYNDEN \& OLDEMAN 1997). Die in den Studien gewählten Entnahmetiefen waren z.T. sehr heterogen. Die mittleren Oberbodenmächtigkeiten betrugen für Profile unter natürlicher Vegetation $23 \mathrm{~cm}( \pm 8, n=191)$ und für Profile unter landwirtschaftlicher Nutzung $21 \mathrm{~cm}( \pm 7, n=179)$. Wurden dem Oberboden zwei Teilproben entnommen, wurden die Werte tiefengewichtet gemittelt. Für die Gewährleistung einer ausreichenden statistischen Datenbasis wurden auch Profile von außerhalb des engeren Untersuchungsgebiets in die Analyse einbezogen.

Die Messmethoden zur Bestimmung von Bodenparametern entsprechen sich weitestgehend. Das erklärt sich dadurch, dass die meisten bodenanalytischen Messungen im Labor von CIAT in Santa Cruz durchgeführt wurden. Abweichungen gab es bei der Bestimmung des Phosphorgehaltes. Am häufigsten wurde die Methode nach OLSEN gewählt, weniger häufig die nach TROUG. Da zwischen beiden Analyseverfahren keine Anpassungsfunktionen existieren, wurden für die Gewährleistung der Vergleichbarkeit nur die nach Olsen gemessenen Werte übernommen. Bei den verwendeten Einheiten gab es, abgesehen von veralteten Schreibweisen, kaum Unterschiede. In wenigen Fällen lagen Elementkonzentrationen in ppm vor. Diese wurden mit Hilfe der Molekularmasse der entsprechenden Elemente in die international gebräuchliche Einheit $\mathrm{cmol}_{\mathrm{c}} \mathrm{kg}^{-1}$ umgerechnet. Lagen keine Angaben zum Gehalt an organischer Substanz vor, wurde dieser durch Multiplikation des Kohlenstoffgehalts mit dem Faktor 1,72 abgeleitet (LANDON 1984).

Tab. 7: Korngrößenklassen nach USDA/FAO und AG Boden

\begin{tabular}{lrr}
\hline Klassifikationssystem & USDA/FAO & AG Boden \\
\hline Sand & $2,0-0,05 \mathrm{~mm}$ & $2,0-0,063 \mathrm{~mm}$ \\
Schluff & $0,05-0,002 \mathrm{~mm}$ & $0,063-0,002 \mathrm{~mm}$ \\
Ton & $<0,002 \mathrm{~mm}$ & $<0,002 \mathrm{~mm}$ \\
\hline
\end{tabular}

Die Einteilung der Korngrößenklassen orientierte sich bei fast allen Studien an dem in Südamerika gebräuchlichen USDA/FAO-Klassifikationssystem (SOIL SURVEY STAFF 2003). Lediglich vier benutzten die in Deutschland gebräuchlichen Klassengrenzen (AG BODEN 1994). 
Tab. 8: Korrelationsmatrix Oberbodenparameter unter natürlicher Vegetation

\begin{tabular}{|c|c|c|c|c|c|c|c|c|c|c|c|}
\hline & & ${ }^{1}$ Tiefe & Sand & Schluff & Ton & pH & $\mathbf{K}$ & KAK & $\mathbf{P}$ & SOM & $\mathbf{N}_{\mathbf{t}}$ \\
\hline \multirow[t]{2}{*}{ Sand } & $\mathrm{r}$ & $-0,26 * * *$ & - & - & - & - & - & - & - & - & - \\
\hline & $\mathrm{n}$ & 191 & - & - & - & - & - & - & - & - & - \\
\hline \multirow[t]{2}{*}{ Schluff } & $\mathrm{r}$ & $0,22 * *$ & $-0,72 * * *$ & - & - & - & - & - & - & - & - \\
\hline & $\mathrm{n}$ & 191 & 191 & - & - & - & - & - & - & - & - \\
\hline \multirow[t]{2}{*}{ Ton } & $\mathrm{r}$ & $0,19 * *$ & $-0,80 * * *$ & $0,29 * * *$ & - & - & - & - & - & - & - \\
\hline & $\mathrm{n}$ & 191 & 191 & 191 & - & - & - & - & - & - & - \\
\hline \multirow[t]{2}{*}{ pH } & $\mathrm{r}$ & n.s & n.s. & $0,26 * * *$ & $-0,15^{*}$ & - & - & - & - & - & - \\
\hline & $\mathrm{n}$ & 191 & 191 & 191 & 191 & - & - & - & - & - & - \\
\hline \multirow[t]{2}{*}{ K } & $\mathrm{r}$ & n.s. & $-0,63 * * *$ & $0,47 * * *$ & $0,54 * * *$ & n.s. & - & - & - & - & - \\
\hline & $\mathrm{n}$ & 173 & 173 & 173 & 173 & 173 & - & - & - & - & - \\
\hline \multirow[t]{2}{*}{ KAK } & $\mathrm{r}$ & n.s. & $-0,66^{* * *}$ & $0,43 * * *$ & $0,66 * * *$ & $0,31 * * *$ & $0,61 * * *$ & - & - & - & - \\
\hline & $\mathrm{n}$ & 160 & 160 & 160 & 160 & 160 & 147 & - & - & - & - \\
\hline \multirow[t]{2}{*}{$\mathbf{P}$} & $\mathrm{r}$ & n.s. & $-0,54 * * *$ & $0,57 * * *$ & $0,44 * * *$ & $0,16^{*}$ & $0,60 * * *$ & $0,54 * * *$ & - & - & - \\
\hline & $\mathrm{n}$ & 131 & 131 & 131 & 131 & 131 & 129 & 129 & - & - & - \\
\hline \multirow[t]{2}{*}{ SOM } & $\mathrm{r}$ & n.s. & $-0,37 * * *$ & $0,15^{*}$ & $0,40 * * *$ & n.s. & $0,29 * * *$ & $0,42 * * *$ & n.s. & - & - \\
\hline & $\mathrm{n}$ & 170 & 170 & 170 & 170 & 170 & 155 & 142 & 115 & - & - \\
\hline \multirow[t]{2}{*}{$N_{t}$} & $\mathrm{r}$ & n.s. & $-0,19 * *$ & n.s. & $0,19 * *$ & n.s. & $0,28 * * *$ & $0,38 * * *$ & n.s. & $0,70 * * *$ & - \\
\hline & $\mathrm{n}$ & 155 & 155 & 155 & 155 & 155 & 143 & 129 & 103 & 155 & - \\
\hline \multirow[t]{2}{*}{$\mathbf{d}_{\mathbf{b}}$} & $\mathrm{r}$ & n.s. & $-0,29 *$ & n.s. & n.s. & n.s. & n.s. & n.s. & n.s. & n.s. & n.s. \\
\hline & $\mathrm{n}$ & 37 & 37 & 37 & 37 & 37 & 31 & 31 & 24 & 34 & 32 \\
\hline
\end{tabular}

Signifikanzniveau $\alpha<0,05 *, \alpha<0,01 * *, \alpha<0,001 * * *$ (n. SPEARMAN, einseitig) - ${ }^{1}$ Entnahmetiefe

Tab. 9: Korrelationsmatrix Oberbodenparameter unter landwirtschaftlicher Nutzung

\begin{tabular}{|c|c|c|c|c|c|c|c|c|c|c|c|c|}
\hline & & ${ }^{1}$ Tiefe & Sand & Schluff & Ton & pH & $\mathbf{K}$ & KAK & $\mathbf{P}$ & SOM & $\mathbf{N}_{\mathbf{t}}$ & $d_{b}$ \\
\hline \multirow[t]{2}{*}{ Sand } & $\mathrm{r}$ & $0,14 *$ & - & - & - & - & - & - & - & - & - & - \\
\hline & $\mathrm{n}$ & 160 & - & - & - & - & - & - & - & - & - & - \\
\hline \multirow[t]{2}{*}{ Schluff } & $\mathrm{r}$ & n.s. & $-0,92 * * *$ & - & - & - & - & - & - & - & - & - \\
\hline & $\mathrm{n}$ & 160 & 160 & - & - & - & - & - & - & - & - & - \\
\hline \multirow[t]{2}{*}{ Ton } & $\mathrm{r}$ & $-0,13 *$ & $-0,82 * * *$ & $0,60 * * *$ & - & - & - & - & - & - & - & - \\
\hline & $\mathrm{n}$ & 160 & 160 & 160 & - & - & - & - & - & - & - & - \\
\hline \multirow[t]{2}{*}{ pH } & $\mathrm{r}$ & $-0,16^{*}$ & n.s. & n.s. & $-0,15^{*}$ & - & - & - & - & - & - & - \\
\hline & $\mathrm{n}$ & 161 & 160 & 160 & 160,00 & - & - & - & - & - & - & - \\
\hline \multirow[t]{2}{*}{$\mathbf{K}$} & $\mathrm{r}$ & n.s. & $-0,44 * * *$ & $0,37 * * *$ & $0,40 * * *$ & $0,21 * *$ & - & - & - & - & - & - \\
\hline & $\mathrm{n}$ & 164 & 145 & 145 & 145 & 146 & - & - & - & - & - & - \\
\hline \multirow[t]{2}{*}{ KAK } & $\mathrm{r}$ & $-0,30 * * *$ & $-0,62 * * *$ & $0,49 * * *$ & $0,61 * * *$ & $0,39 * * *$ & $0,57 * * *$ & - & - & - & - & - \\
\hline & $\mathrm{n}$ & 134 & 134 & 134 & 134 & 134 & 130 & - & - & - & - & - \\
\hline \multirow[t]{2}{*}{$\mathbf{P}$} & $\mathrm{r}$ & $-0,21 * *$ & n.s. & n.s. & n.s. & $0,50 * * *$ & $0,57 * * *$ & $0,42 * * *$ & - & - & - & - \\
\hline & $\mathrm{n}$ & 144 & 143 & 143 & 143 & 144 & 143 & 128 & - & - & - & - \\
\hline \multirow[t]{2}{*}{ SOM } & $\mathrm{r}$ & n.s. & $-0,48 * * *$ & $0,41 * * *$ & $0,50 * * *$ & $0,21^{*}$ & $0,60 * * *$ & $0,75 * * *$ & $0,35 * * *$ & - & - & - \\
\hline & $\mathrm{n}$ & 140 & 121 & 121 & 121 & 122 & 125 & 102 & 105 & - & - & - \\
\hline \multirow[t]{2}{*}{$\mathbf{N}_{\mathrm{t}}$} & $\mathrm{r}$ & n.s. & $-0,52 * * *$ & $0,46^{* * *}$ & $0,50 * * *$ & n.s. & $0,60 * * *$ & $0,70 * * *$ & $0,32 * * *$ & $0,90 * * *$ & - & - \\
\hline & $\mathrm{n}$ & 156 & 138 & 138 & 138 & 138 & 155 & 123 & 135 & 117 & - & - \\
\hline \multirow[t]{2}{*}{$\mathbf{d}_{\mathbf{b}}$} & $\mathrm{r}$ & $0,42 * *$ & $0,33^{*}$ & $-0,24 *$ & $-0,42 * *$ & $-0,47 * * *$ & $-0,53 * * *$ & $-0,81 * * *$ & $-0,41 * *$ & $-0,72 * * *$ & $-0,67 * * *$ & - \\
\hline & $\mathrm{n}$ & 48 & 48 & 48 & 48 & 48 & 36 & 31 & 37 & 48 & 36 & - \\
\hline Dauer der & $\mathrm{r}$ & n.s. & $0,44 * * *$ & $-0,38 * * *$ & $-0,44 * * *$ & n.s. & $-0,43 * * *$ & $-0,33 * * *$ & n.s. & $-0,58 * * *$ & $-0,49 * * *$ & $0,69 * * *$ \\
\hline Nutzung & $\mathrm{n}$ & 127 & 108 & 108 & 108 & 109 & 114 & 92 & 94 & 127 & 106 & 47 \\
\hline
\end{tabular}

Signifikanzniveau $\alpha<0,05 *, \alpha<0,01 * *, \alpha<0,001 * * *$ (n. SPEARMAN, einseitig) - ${ }^{1}$ Entnahmetiefe 
Abweichungen zwischen beiden Systemen liegen in unterschiedlichen Sand-Schluff Grenzen, die Schluff-Ton Grenze ist gleich (Tab.7). Da es bislang keine standardisierten Verfahren für die Übertragung von Werten auf das jeweils andere System gibt, wurde auf eine Anpassung verzichtet. Verschiedene Autoren, die sich mit der Standardisierung und Vergleichbarkeit von Texturdaten unterschiedlicher Systeme beschäftigt haben, kommen zu dem Ergebnis, dass es bis heute keine allgemein gültige und zuverlässige Lösung für dieses Problem gibt. Aufgrund der Vielzahl nationaler Systeme und der damit verbundenen Schwierigkeit der Vergleichbarkeit, wäre die Festlegung auf ein international standardisiertes System wünschenswert (MinASNy \& MCBRATNEY 2001, NEMES et al. 1999, RousSEVA 1997, SHIRAZI et al. 2001). Die Angaben der Korngrößenverteilung lagen weitgehend in Prozent für Sand, Schluff und Ton vor; eine Unterteilung in die jeweiligen Unterfraktionen bestand bei 54 Profilen. Messungen der Unterfraktionen wurden insgesamt nur wenige durchgeführt. Für die Expansionszone bspw. existieren keine Analysen der Unterfraktionen (GUAMAN mündl. Mitt.). Lagen anstelle von Prozentangaben die Bodenartenkürzel der Korngrößenklassen vor, erfolgte eine Metrisierung der Werte über die Klassenmitten (Abb.A1, Anhang). Die Regionalisierung der Bodenarten wurde auf Grundlage der drei Größenklassen Sand, Schluff und Ton durchgeführt (Kap.5.1.1). Zur Abschätzung der Winderosionsgefährdung wurden die Unterfraktionen rechnerisch abgeleitet und unter Einbezug des Humusgehaltes die Erodibilität der Böden über die Bestimmung von K-Faktoren berechnet (Kap. 5.1.4.3).

Die Profile wurden in Zustand unter natürlicher Vegetation und anthropogen überformten Zustand (Profile unter landwirtschaftlicher Nutzung) unterteilt und, sofern den Studien zu entnehmen, Art und Dauer der Agrarnutzung zum Zeitpunkt der Aufnahme angegeben. Bestanden keine Hinweise über die Dauer der Nutzung, wurde eine Abschätzung auf Grundlage der Satellitenbilder vorgenommen. Derart konnte den meisten Agrarflächen eine Angabe über den Zeitraum ihrer jeweiligen Nutzung zugewiesen werden.

\section{Korrelationen zwischen Bodeneigenschaften}

Die in der Datenbank integrierten Bodeneigenschaften wurden über eine Korrelationsanalyse auf ihre Plausibilität geprüft. Die Tabellen 8 und 9 zeigen zahlreiche signifikante Korrelationen zwischen verschiedenen Bodeneigenschaften. Der organische Kohlenstoff im Boden (SOC) ist in den Tabellen nicht explizit aufgeführt, da aus den SOC-Gehalten die organische Substanz (SOM) durch einen einheitlichen Faktor $(1,72)$ errechnet wurde und sich demnach gleiche Korrelationen ergeben.

Die Kationenaustauschkapazität (KAK) der Böden wird durch die Körnung (Tongehalt), die organische Substanz und den pH-Wert bestimmt. Sinken die SOM-Gehalte, nimmt gleichzeitig die KAK ab (BuRle et al. 1997, KATYAL et al. 2001). Die Funktion der organischen Substanz als Nährstoffpool wird über positive Korrelationen zu den Pflanzennährstoffen deutlich. Die Gehalte an organischer Substanz und damit auch die N-, P- und K- 
Gehalte nehmen von der Sand- zur Tonfraktion zu. Die organische Substanz hat entscheidenden Anteil an der Formation und Stabilisierung von Bodenaggregaten. Besonders in sandigen Böden kommt dieser dabei zentrale Bedeutung zu (DALAL \& CHAN 2001). Mit zunehmender Nutzungsdauer nehmen die Gehalte an organischer Substanz und damit auch die KAK und die Nährstoffgehalte (N, K) ab. Bodenbearbeitung bricht Aggregate auf und setzt organische Substanz frei (SIX et al. 1999). Diese wird unter den gegebenen klimatischen Bedingungen rasch mineralisiert und zusätzlich durch den Entzug von Biomasse durch Ernte bzw. die Beweidung oder das Abrennen der Ernterückstände in nur wenigen Jahren auf ein niedriges Niveau gebracht (DOMINY et al. 2002). Beim Phosphor ist kein Trend in Abhängigkeit von der Nutzungsdauer zu erkennen. SAIKH et al. (1998) begründen dies mit dem höheren Biomasseanteil geschlossener Wälder und einer damit verbundenen höheren PAufnahme gegenüber Kulturflächen. Wahrscheinlich spielt auch die sehr hohe Varianz der PGehalte in der Alluvialebene eine Rolle (Tab.2), weshalb keine Abhängigkeiten nachweisbar sind. Denkbar wäre zudem, dass P im Untersuchungsraum z.T. nicht organischen Ursprungs ist. Nach Angaben von KATYAL et al (2001) liegt der SOM-bürtige Phosphoranteil im Boden zwischen 20-80 \%. Positiv mit der Dauer der Nutzung korreliert sind die Sandgehalte und Lagerungsdichten. Bei beiden geht der Anstieg signifikant mit einer Abnahme der organischen Substanz einher. Abnehmende Gehalte organischer Substanz bedeuten eine Abnahme der Aggregatstabilität und mithin der Stabilität des Bodengefüges, der Boden verdichtet leichter (DAVIDSON ACKERMANN 1993, KOZLOWSKI 1999). Gleichzeitig sind durch die Auflösung von Aggregaten die feinen Kornfraktionen leichter erodierbar. Einen durch Kultivierung bedingten Anstieg der Sandgehalte im Oberboden konstatieren zahlreiche Autoren (BRAIMOH \& VLEK 2004, JAIYEOBA 2003, LAL 1996a, ZHAO et al. 2005).

Die deskriptive Statistik der Bodeneigenschaften unter natürlicher Vegetation nach Regionen ist in Tabelle 2 und Abbildung 4 (Kap.2) dargestellt.

\section{Potenzielle Fehlerquellen}

Naturgemäß können bei der Integration von Werten aus verschiedenen Studien in einer Datenbank Unstimmigkeiten auftreten. Demnach müssen die abgeleiteten Ergebnisse als relative Größenordungen eingeordnet werden. Systematische Fehler, wie diese bspw. von der Probennahme bis zur Analytik auftreten können, sind im Nachhinein nicht mehr nachvollziehbar. Potenzielle Fehlerquellen sind zudem in der Gegenüberstellung von Analysewerten aus unterschiedlichen Entnahmetiefen und in den z.T. unpräzisen Lokalisationen der Bodenprofile zu sehen. Speziell in den älteren Studien wurde oft nur eine grobe Lagebeschreibung der Profile angegeben. Ungenaue Verortungen indes bergen bei der Regionalisierung von Bodeneigenschaften die Gefahr, keine oder fehlerhafte Zusammenhänge zwischen zu schätzender Bodeneigenschaft und Klima- und Reliefvariablen zu ermitteln. Dieser Fehler verringert sich mit abnehmender Auflösung des digitalen Geländemodells. 
Unterschiedliche Entnahmetiefen bedingen in der Regel unterschiedliche Elementkonzentrationen. Allerdings ist nach Mittelung der Oberbodenwerte in dieser Hinsicht kein eindeutiger Trend zu erkennen (Tab.8, 9). Für eine bessere Vergleichbarkeit von Elementkonzentrationen schlagen MURTY et al. (2002) einheitliche Entnahmetiefen bei zukünftigen Studien vor. Eine unsichere Größe bei den landwirtschaftlichen Profilen ist die über die Satellitenbilder geschätzte Dauer der ackerbaulichen Nutzung, da die zum Zeitpunkt der Bodenaufnahme gemessenen Werte nicht zwangsläufig auf kontinuierlichen Ackerbau zurückzuführen sind. Möglich wären z.B. Elementanreicherungen während eines Brachestadiums. Gleichwohl dürfte diese Fehlerquelle gering sein, da vor allem in den 1980er und 1990er Jahren kaum ausreichende Brachezeiten für eine adäquate Nährstoffregeneration eingehalten wurden (GEROLD 1986, DAVIES 1992).

Insgesamt ist die Datengrundlage des Bodens in Bezug zur Größe des Untersuchungsraums gering. Zahlreichen Einzeluntersuchungen steht eine nur kleine Anzahl an Übersichtskartierungen gegenüber (AGRAR- \& HYDROTECHNIK 1973, 1974a, 1974b, GUAMAN 1988a, 1988b, GuAMAn 1999, IPORRE BELlido 1996). Diese jedoch wären nötig, um eine höhere räumliche Repräsentativität der Daten zu erzielen. Schon BARBER (1995) weist auf darauf hin, dass für die Region wenig Bodenuntersuchungen existieren, insbesondere solche, die sich mit potenziellen Auswirkungen der Landnutzungssysteme auf den Boden beschäftigen.

\subsection{Ableitung von Reliefparametern}

Ausgehend von der Tatsache, dass die Topographie einen wesentlichen Einfluss auf die Bodenbildung besitzt (JENNY 1941), hat sich die Reliefanalyse bei der räumlichen Schätzung von Bodeneigenschaften zu einem nützlichen Werkzeug entwickelt (MCBRATNEY et al. 2003). Diesem Verfahren liegt das Prinzip zugrunde, flächenhafte Reliefparameter als Schätzvariablen für die Regionalisierung punktuell vorliegender Bodendaten zu nutzen (Kap.5.1.1). Dabei kommen statistische und geostatistische Verfahren zum Einsatz, die Zusammenhänge überprüfen und Schätzungen metrischer Bodeneigenschaften vornehmen (BÖHNER et al. 2003). Das in vorliegender Studie verwendete digitale Geländemodell (DGM) stammt aus der Shuttle Radar Topography Mission (SRTM) und ist kostenfrei über das Internet verfügbar ${ }^{9}$. Das DGM hat eine räumliche Auflösung von 92,5 m. Um die Rechenoperationen für den Untersuchungsraum praktikabel zu halten, wurde das DGM auf eine Rasterweite von $185 \times 185$ m resampelt.

Die Reliefanalyse basiert auf der Ableitung topographischer Eigenschaften, die allgemein in primäre und sekundäre Reliefparameter unterteilt werden. Primäre Reliefparameter werden direkt aus dem DGM berechnet und umfassen lokale (z.B. Hangneigung, Exposition) und komplexe bzw. regionale Reliefparameter (z.B. Einzugsgebietsgröße einer Rasterzelle, Höhe

\footnotetext{
9 ftp://e0mss21u.ecs.nasa.gov/srtm/
} 
über Tiefenlinie). Letztgenannte berücksichtigen im Gegensatz zu lokalen Reliefparametern größere räumliche Zusammenhänge innerhalb der Nachbarschaft einer Rasterzelle (MCBRATNEY et al. 2003). Sekundäre Reliefparameter (z.B. Bodenfeuchte-Index) beschreiben anhand prozessorientierter oder empirischer Gleichungen unter Kombination primärer Reliefparameter die räumliche Differenzierung wichtiger, die Bodenbildung beeinflussender Prozesse, bspw. Abfluss- und Umlagerungsprozesse (WILSON \& Gallant 2000). Im folgenden werden die aus dem DGM abgeleiteten komplexen Reliefparameter kurz beschrieben. Bezüglich der mathematischen Formulierungen der Algorithmen sei auf BÖHNER \& SELIGE (2004) verwiesen.
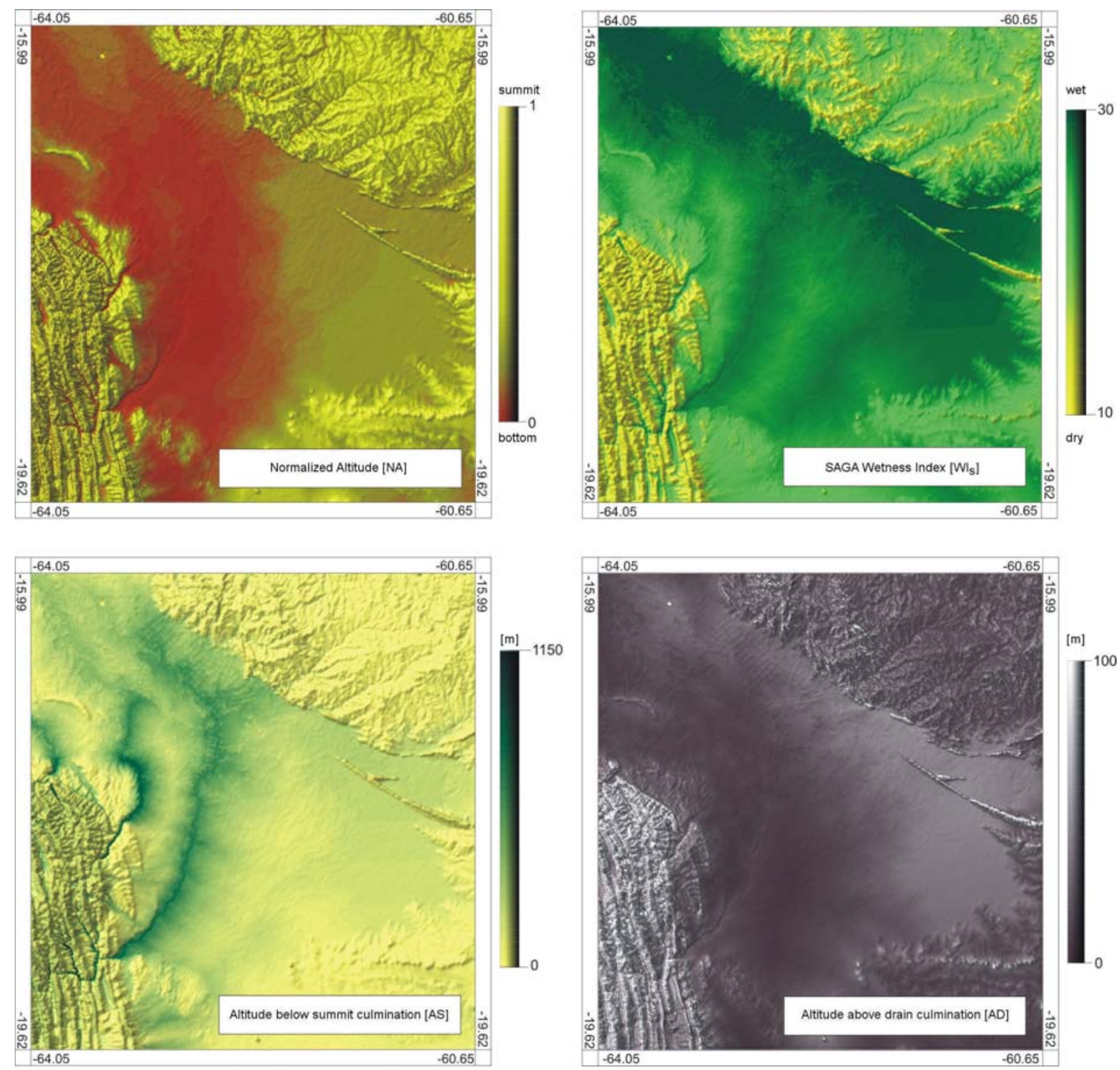

Abb. 9: Sekundäre Reliefparameter 
Der SAGA-Bodenfeuchte-Index $\left[\mathrm{WI}_{\mathrm{S}}\right]$ errechnet sich in Abhängigkeit vom Neigungswinkel und spezifischer Einzugsgebietsgröß $\mathrm{e}^{10}$ einer Rasterzelle und findet als Schätzvariable der hypothetischen Feuchteverteilung im Boden Verwendung. Der ursprünglich von MoORE et al. (1993) entwickelte Algorithmus wird beim $\mathrm{WI}_{\mathrm{S}}$ durch Anwendung einer Iteration modifiziert. Dabei wird die spezifische Einzugsgebietsgröße in einer neigungsabhängigen Funktion für jede Rasterzelle solange neu berechnet bis das Ergebnis konstant bleibt. Diese Neuformulierung liefert im Gegensatz zum ursprünglichen Algorithmus auch in ebenem Gelände plausible Ergebnisse und bildet damit eine entscheidende Voraussetzung für deren begründbare Anwendung im Untersuchungsgebiet. Die Parameter Höhe über Tiefenlinie [AD] und Höhe unter Scheitellinie [AS] beschreiben auf Basis der Reliefgeometrie und der Einzugsgebietsgröße relative Lagebeziehungen der Rasterzellen zu den jeweiligen lokalen Erosionsbasen bzw. Scheitelpunkten. Aus beiden Parametern leitet sich die normierte Höhe [NA] ab, die die relative Hangposition einer Rasterzelle berechnet. Alle drei Parameter differenzieren Art und Ausmaß potenzieller Umlagerungs- und Abflussprozesse.

\subsection{Ableitung von Klimaparametern}

Das Konzept Klimaflächendaten als Schätzvariablen räumlicher Bodeneigenschaften zu nutzen wird, obwohl der klimatische Einfluss auf Bodenbildungsprozesse unbestritten ist, bis dato wenig angewendet. Nach Angaben im Review von MCBRATNEY et al. (2003) nutzten bei der Schätzung von Bodeneigenschaften lediglich $5 \%$ der begutachteten Studien zusätzlich Klimavariablen, während am häufigsten Reliefparameter die Basis der Regionalisierung bilden $(80 \%)$.

Der regionalen Klimaanalyse lag ein Datensatz aus 469 Klimamessstationen für ganz Bolivien mit den Monatsmitteln von Niederschlag (307 Stationen) und Temperatur (197 Stationen) zugrunde, der freundlicherweise von RAFIQPOOR et al. (2004) zur Verfügung gestellt wurde. Die Messzeiträume liegen im Mittel bei 21 Jahren mit einer Spannweite von 90 Jahren. Über einen semi-empirischen Regionalisierungsansatz wurden die an den Stationen erhobenen Klimaparameter mit allgemeinen Zirkulationsdaten (GCM) und digitalen Geländedaten (SRTM-DGM, $925 \mathrm{~m}$ ) in Beziehung gesetzt und Flächendaten von Niederschlag, Temperatur und Strahlung abgeleitet (Abb.10) ${ }^{11}$. Dem Konzept zugrunde liegt ein downscaling Verfahren, bei dem über die Ableitung statistischer Beziehungen zwischen beobachteten Stationsdaten und großräumigen Zirkulationsverhältnissen Informationen auf die regionale Skala transformiert werden. Grundlage der Zirkulationsdaten bilden Reanalyse-

\footnotetext{
${ }^{10}$ Die spezifische Einzugsgebietsgröße bezeichnet die einer Rasterzelle zugehörige Abflussfläche bezogen auf eine normierte Breite $\left[\mathrm{m}^{2} \mathrm{~m}^{-1}\right]$. Siehe hierzu z.B. TARBOTON (2003).

${ }^{11}$ Die Klimaregionalisierung wurde freundlicherweise von PD Dr. J. BÖHNER durchgeführt.
} 
Daten $^{12}$ des US-amerikanischen National Centre for Environmental Prediction (NCEP) und dem National Centre for Atmospheric Research (NCAR) die ebenfalls kostenlos über das Internet zu beziehen sind ${ }^{13}$. Für ausführliche Informationen über das Reanalyse-Verfahren sowie die Qualität der Daten sei auf KALNAY et al. (1996) verwiesen. Davon ausgehend, dass zeitliche und räumliche Klimavariabilitäten topographischen Einflüssen unterliegen, wurden Zirkulationsvariablen mit Reliefparametern statistisch verknüpft und Klimaparameter regionalisiert. Das methodische Konzept der Klimaregionalisierung wird ausführlich bei BÖHNER (2004) beschrieben.

Precipitation

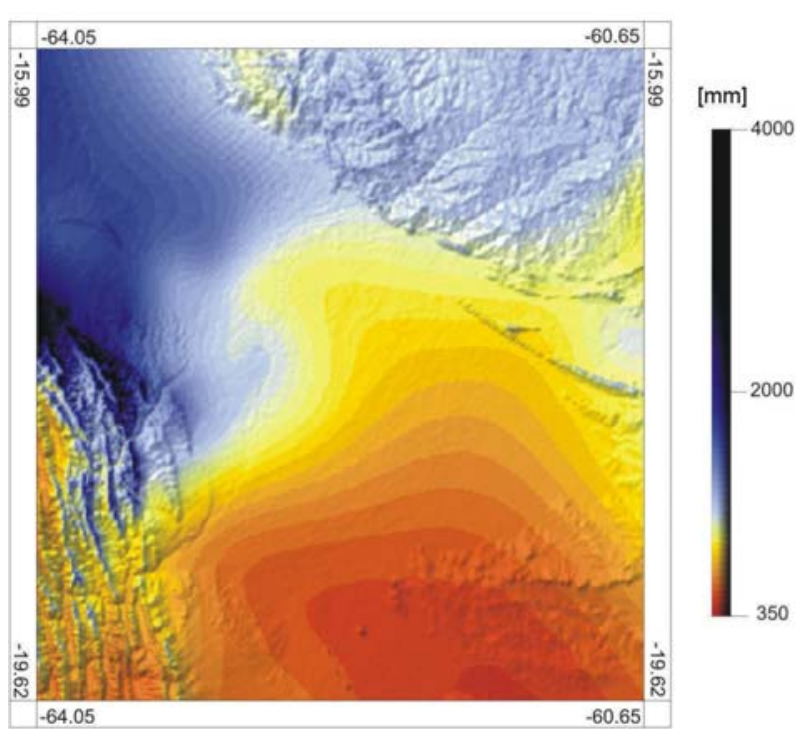

Solar Radiation

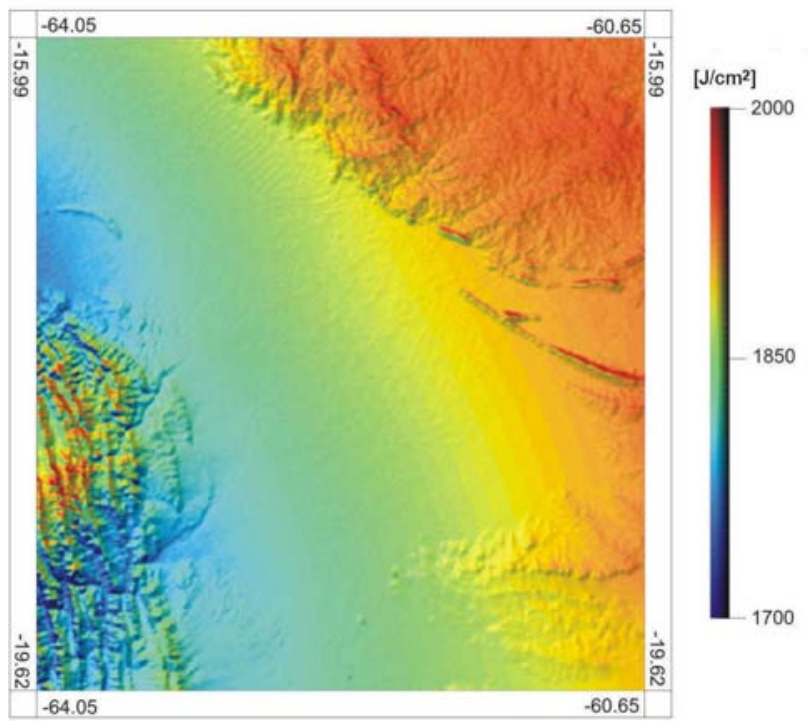

Temperature

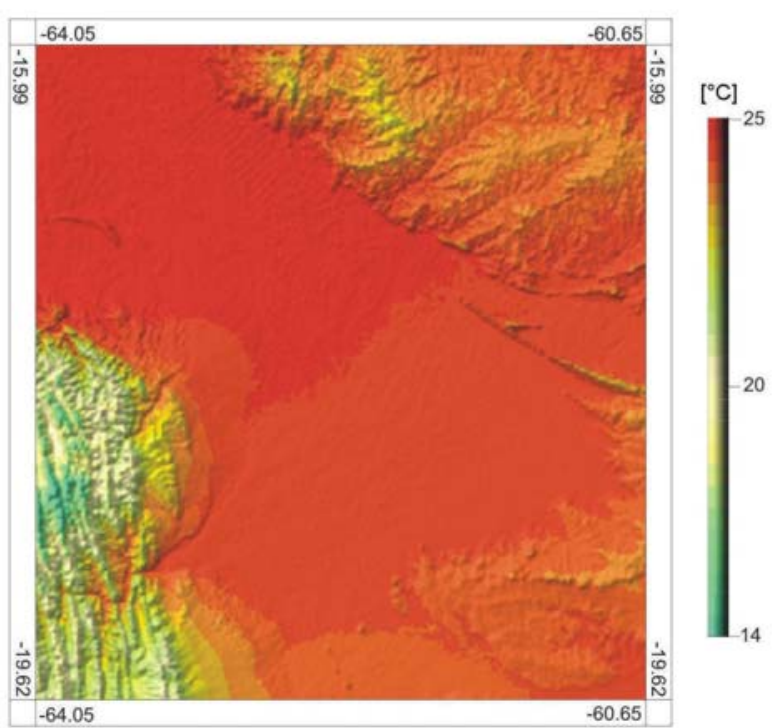

Abb. 10: Klimavariablen Niederschlag, Temperatur, Strahlung

\footnotetext{
${ }^{12}$ Reanalyse-Daten sind Ergebnisse eines Klimamodells, in dem Atmosphärendaten nach einem einheitlichen Verfahren anhand empirischer Daten fortlaufend korrigiert werden. Die Daten liegen seit 1948 global mit einer räumlichen Auflösung von etwa $250 \mathrm{~km}$ vor.

${ }^{13} \mathrm{http} / / /$ www.cdc.noaa.gov/ncep_reanalysis
} 


\section{WALDKONVERSION}

\subsection{Waldkonversion im bolivianischen Tiefland}

Angaben der FAO (2003) zufolge lag die durchschnittliche Abholzungsrate der letzten zehn Jahre in Bolivien bei $0,3 \%$ pro Jahr und damit leicht unter dem südamerikanischen Mittel von 0,4\%. Im Vergleich mit den Ergebnissen einer staatlichen Studie, kann für die letzten 30 Jahre von einer konstanten Rate, entsprechend einem mittleren Waldflächenverlust von $1.700 \mathrm{~km}^{2}$ pro Jahr, ausgegangen werden (MDSMA 1995). Befanden sich die Abholzungsraten im bolivianischen Tiefland angesichts der geringen Besiedlungsdichte dieses Raums lange Zeit auf niedrigem Niveau, so änderte sich der Trend mit der Umsetzung staatlicher Kolonisationsprogramme bis Mitte der 1980er Jahre zunächst graduell und anschließend, infolge der Umsetzung neuer ökonomischer Richtlinien, drastisch (Kap.2.3.1).

Bezogen auf die Landesfläche Boliviens zeigen sich die aktuell höchsten Waldkonversionsraten im tropischen Tiefland mit einer räumlichen Konzentration im Department Santa Cruz (Tab.10). Entfielen Ende der 1980er Jahre ca. 40 \% der Abholzungen im bolivianischen Amazonasraum ${ }^{14}$ auf das Department Santa Cruz, waren es Mitte der 1990er Jahre bereits mehr als 60 \% (CUMAT 1992, STEININGER et al. 2001a). Diese Tendenz belegen Abholzungssummen, die Ende der 1980er Jahre jährlich rund $700 \mathrm{~km}^{2}$ betrugen, 10 Jahre später aber bereits bei über $2.000 \mathrm{~km}^{2}$ lagen (CAMACHO et al. 2001, CUMAT 1992, MORALES 1993). Innerhalb des Departments wiederum konzentriert sich die stärkste Rodungsaktivität auf die Hauptlandwirtschaftszone ${ }^{15}$ um Santa Cruz. Dort steigerte sich der jährliche Anteil im gleichen Zeitraum von ca. $400 \mathrm{~km}^{2}$ auf knapp $1.000 \mathrm{~km}^{2}$, entsprechend einer geschätzten mittleren Waldkonversion von $3 \%$ pro Jahr (BOUNOUA et al. 2003, CAMACHO et al. 2001, CORDECRUZ et al. 1994, MORALEs 1993, STEININGER et al. 2001b, eigene Untersuchungen). Den in Tab.10 dargestellten Ergebnissen zufolge befinden sich mehr als die Hälfte aller Rodungsflächen des Departments in dieser Region, deren Flächenanteil im Department weniger als $20 \%$ beträgt. Gleiches bestätigen Untersuchungen von CAMACHO et al. (2001), die in 5 von insgesamt 35 untersuchten Munizipen ${ }^{16}$ annähernd $70 \%$ der im Zeitraum von 1993 bis 2000 ermittelten Rodung lokalisieren. STEININGER et al. (2001b) konstatieren für diesen relativ begrenzten Raum eine der höchsten Abholzungsraten weltweit.

In zeitlicher Hinsicht ist die stärkste Rodungsaktivität im bolivianischen Tiefland mit einem Anteil von annähernd $40 \%$ bis Mitte der 1980er Jahre in den Kolonisationsgebieten der zentralen Zone zu verzeichnen (TUCKER \& TOWNSHEND 2000). Die staatliche Förderung großflächiger industrieller Landwirtschaft führte in den 1990er Jahren zu einer Verlagerung in

\footnotetext{
${ }^{14}$ Bezeichnet den Raum nördlich $19^{\circ}$ südl. Breite (STEININGER et al. 2001a, TUCKER \& TownSHEND 2000), bzw. nördlich $18^{\circ}$ südl. Breite (CUMAT 1992).

${ }^{15}$ Bezieht sich auf die zentrale Zone und Expansionszone.

${ }^{16}$ Insgesamt unterteilt sich das Department Santa Cruz in 50 Munizipe (INE 2002).
} 
die Expansionszone, in der innerhalb von nur 8 Jahren mehr als $7.000 \mathrm{~km}^{2}$ Wald gerodet wurden (STEININGER et al. 2001b).

CAMACHO et al. (2001) ermittelten in nur zwei Munizipen der Expansionszone (ca. $5 \%$ der Departmentfläche) einen Anteil von 35 \%an allen Rodungsflächen im Department. Die höchsten Abholzungsraten datieren aus Mitte der 1990er Jahre und zeigen danach, als Folge wachsender ökonomischer Schwierigkeiten, einen allmählichen Rückgang (BouNOUA et al. 2003, STEININGER et al. 2001b). Bis heute wurden ca. $10 \%\left(\sim 30.000 \mathrm{~km}^{2}\right)$ der ursprünglichen Waldfläche im Department Santa Cruz gerodet. Die durchschnittliche Rate der letzten zehn Jahre lag mit 0,6 \% doppelt so hoch wie der Landesdurchschnitt. Der überwiegende Anteil der Konversionen findet zugunsten von Ackerland statt, ein kleinerer Teil der Rodungsflächen, vornehmlich im Nordosten, wird zur Viehzucht genutzt (Kap.2.3). Die Vorgaben des 1996 publizierten staatlichen Landnutzungsplans PLUS (CORDECRUZ et al. 1996) finden insgesamt wenig Umsetzung. Dies zeigt sich daran, dass ca. 42 \% der bis 2000 im Department gerodeten Flächen ursprünglich einer Forstnutzung vorbehalten sein sollten, bzw. als Schutzzonen ausgewiesen waren (CAMACHO et al. 2001). Insbesondere Rodungen entlang der Schutzzonen von Gewässerläufen und die Praxis kleinere Bachläufe und Feuchtgebiete zuzuschütten und für eine landwirtschaftliche Nutzung einzuebnen, haben einer Studie von WACHHOLTZ \& Herold-Mergl (2003) zufolge die Frequenz folgenschwerer Hochwässer entlang des Río Grande in den letzten Jahrzehnten erhöht.

\subsection{Waldkonversion im Untersuchungsgebiet}

Ein wichtiger Faktor im Zusammenhang mit der Evaluation von Bodendegradation besteht in der Erhebung von Landnutzungsinformationen. Um dem übergeordneten Ziel Rechnung zu tragen, werden für das engere Untersuchungsgebiet nachfolgend die Entwicklung der Landnutzung in den letzten zwei Jahrzehnten analysiert sowie die angewendeten Landnutzungssysteme regional klassifiziert. Zu diesem Zweck wurden 6 multispektrale Landsat-Satellitenbilder der Jahre 1984, 1992 und 2001 ausgewertet (jeweils 2 pro Jahr). Damit ergibt sich ein 17-jähriger Untersuchungszeitraum, der sich für die Analyse der Veränderung von Bodeneigenschaften durch Kultivierung (Kap.5) grob in drei Nutzungsperioden einteilen lässt: $<10$ a, 10-20 a, $>20$ a. Bei der Auswahl der Satellitenbilder wurde weiterhin darauf geachtet, dass diese den größten Teil der Hauptlandwirtschaftszone des Departments Santa Cruz abdecken. Zudem sollten alle Landnutzungssysteme, sowie ein Teil der alten landwirtschaftlichen Entwicklungszone (zentrale Zone) und insbesondere die Expansionszone, als die derzeit dynamischste Region der landwirtschaftlichen Entwicklung im Department, über die Aufnahmen abgebildet sein (Abb.8). 
Tab. 10: Waldkonversion in Bolivien

\begin{tabular}{|c|c|c|c|c|c|c|c|c|c|c|c|}
\hline \multirow[t]{3}{*}{ Region } & \multirow[t]{3}{*}{ Periode } & \multirow{3}{*}{$\begin{array}{c}\text { Gebiet } \\
\mathrm{km}^{2}\end{array}$} & \multirow{2}{*}{\multicolumn{2}{|c|}{ Wald }} & \multicolumn{6}{|c|}{ Entwaldung } & \multirow{3}{*}{$\begin{array}{c}\text { Anteil } \\
{[\%]}\end{array}$} \\
\hline & & & & & \multicolumn{2}{|c|}{ gesamt } & \multicolumn{2}{|c|}{ Periode } & \multicolumn{2}{|c|}{ jährlich } & \\
\hline & & & $\mathrm{km}^{2}$ & {$[\%]$} & $\mathrm{km}^{2}$ & {$[\%]$} & $\mathrm{km}^{2}$ & {$[\%]$} & $\mathrm{km}^{2}$ & {$[\%]$} & \\
\hline Bolivien ${ }^{1}$ & $1990-2000$ & 1.084 .380 & 530.680 & 49 & $*$ & $*$ & 16.100 & 1 & 1.610 & 0,3 & $*$ \\
\hline Bolivien $^{2}$ & $1975-1993$ & 1.098 .581 & 534.440 & 49 & 54.000 & 5 & 30.240 & 3 & 1.680 & 0,3 & $*$ \\
\hline boliv. Amazonasraum & $1987-1993$ & 731.717 & 437.904 & 60 & 24.703 & 3 & 9.171 & 1 & 1.529 & 0,3 & 100 \\
\hline Dep Santa Cruz ${ }^{3}$ & $1987-1993$ & 354.494 & 213.985 & 60 & 16.933 & 5 & 5.745 & 2 & 958 & 0,4 & 63 \\
\hline boliv. Amazonasraum & $1985-1990$ & 595.660 & 402.540 & 68 & 23.980 & 4 & 3.750 & 1 & 750 & 0,2 & 100 \\
\hline Dep. Santa Cruz ${ }^{4}$ & $1985-1990$ & 224.690 & 166.480 & 74 & 13.760 & 6 & 1.600 & 1 & 320 & 0,2 & 43 \\
\hline Dep. Santa Cruz & 1989-1992 & 363.806 & 309.150 & 85 & 18.607 & 5 & 2.352 & 1 & 784 & 0,3 & 100 \\
\hline & 1992-1994 & 363.806 & 306.797 & 84 & 20.961 & 6 & 2.355 & 1 & 1.177 & 0,4 & 100 \\
\hline zentrale Zone & 1989-1992 & 34.503 & 22.086 & 64 & 9.116 & 26 & 583 & 2 & 194 & 0,9 & 25 \\
\hline & 1992-1994 & 34.503 & 21.503 & 61 & 9.463 & 27 & 347 & 1 & 173 & 0,8 & 15 \\
\hline Expansionszone 5 & 1989-1992 & 35.118 & 30.517 & 87 & 3.729 & 11 & 726 & 2 & 242 & 0,8 & 31 \\
\hline & 1992-1994 & 35.118 & 29.845 & 83 & 4.562 & 13 & 832 & 2 & 416 & 1,4 & 35 \\
\hline Dep. Santa Cruz & 1989-1994 & 364.615 & 309.342 & 85 & $*$ & $*$ & 5.117 & 1 & 1.023 & 0,3 & $*$ \\
\hline zentrale Zone & 1989-1994 & 25.066 & 14.657 & 58 & $*$ & $*$ & 1.129 & 5 & 226 & 1,5 & $*$ \\
\hline Expansionszone $^{6}$ & 1989-1994 & 16.262 & 11.691 & 72 & $*$ & $*$ & 2.057 & 13 & 411 & 3,5 & $*$ \\
\hline Dep. Santa Cruz & $1993-2000$ & 349.909 & 319.684 & 91 & 29.895 & 9 & 14.240 & 4 & 2.034 & 0,6 & 100 \\
\hline zentrale Zone & $1993-2000$ & 35.155 & 22.511 & 64 & 12.616 & 36 & 2.892 & 8 & 413 & 1,8 & 20 \\
\hline Expansionszone $^{7}$ & $1993-2000$ & 16.759 & 8.570 & 51 & 8.185 & 49 & 5.029 & 30 & 718 & 8,4 & 35 \\
\hline zentrale und & $1975-1986$ & 31.208 & 28.521 & 91 & $*$ & $*$ & 859 & 3 & 78 & 0,3 & $*$ \\
\hline Expansionszone $^{8}$ & 1986-1992 & 31.208 & 26.310 & 84 & $*$ & $*$ & 2.211 & 7 & 369 & 1,4 & $*$ \\
\hline & $1992-1996$ & 31.208 & 22.777 & 73 & $*$ & $*$ & 3.533 & 11 & 883 & 3,9 & $*$ \\
\hline & 1996-1999 & 31.208 & 21.225 & 68 & $*$ & * & 1.552 & 5 & 517 & 2,4 & $*$ \\
\hline & 1975-1999 & 31.208 & 21.225 & 68 & $*$ & $*$ & 8.155 & 26 & 340 & 1,6 & $*$ \\
\hline Expansionszone $^{9}$ & $1975-1991$ & 15.650 & * & $*$ & $*$ & $*$ & 2.600 & 17 & 163 & 1,0 & $*$ \\
\hline Expansionszone ${ }^{10}$ & $1975-1984$ & 19.533 & $*$ & $*$ & $*$ & $*$ & 783 & 4 & 87 & 0,5 & $*$ \\
\hline & $1984-1990$ & 19.533 & $*$ & * & $*$ & $*$ & 990 & 5 & 165 & 0,8 & $*$ \\
\hline & 1990-1998 & 19.533 & * & * & $*$ & $*$ & 7.120 & 36 & 890 & 4,6 & $*$ \\
\hline & $1975-1998$ & 19.533 & * & * & $*$ & $*$ & 9.400 & 48 & 409 & 2,1 & $*$ \\
\hline & 1996-1998 & 19.533 & $*$ & $*$ & $*$ & $*$ & 1.924 & 10 & 962 & 4,9 & $*$ \\
\hline 231/72 (Landsat) & 1989-1991 & 17.215 & 8.372 & 49 & 8.798 & 51 & 472 & 3 & 236 & 2,8 & $*$ \\
\hline $230 / 72$ (Landsat) $^{11}$ & 1989-1991 & 12.555 & 9.380 & 75 & 3.155 & 25 & 890 & 7 & 445 & 4,7 & $*$ \\
\hline boliv. Amazonasraum & $1985-1986$ & 789.818 & 445.778 & 56 & 16.410 & 2 & $*$ & $*$ & * & $*$ & 100 \\
\hline 231/72 (Landsat) & $1985-1986$ & 26.430 & 15.811 & 60 & 6.542 & 25 & $*$ & $*$ & $*$ & $*$ & 40 \\
\hline 230/72 (Landsat) & $1985-1986$ & 26.191 & 21.327 & 81 & 2.025 & 8 & $*$ & $*$ & $*$ & $*$ & 12 \\
\hline 230/73 (Landsat) & $1985-1986$ & 26.236 & 6.880 & 26 & 693 & 3 & $*$ & $*$ & $*$ & $*$ & 4 \\
\hline $230 / 73$ (Landsat) $^{12}$ & $1985-1993$ & 26.236 & 6.240 & 24 & 1.334 & 5 & 1.334 & 5 & 167 & 2,7 & $*$ \\
\hline
\end{tabular}

(eigene Bearbeitung)

Quellen: ${ }^{1}$ FAO (2003), ${ }^{3}$ Steininger et al. (2001a), ${ }^{6}$ Mertens et al. (2004), ${ }^{7}$ CAMACho et al. (2001), ${ }^{8}$ Bounoua et al. (2003), ${ }^{10}$ STEININGER et al. (2001b), ${ }^{11}$ CORDECRUZ et al. (1994), ${ }^{12}$ TUCKER \& Townshend (2000); Angaben aus PACHECO (1998): ${ }^{2}$ MDSMA (1995), ${ }^{4}$ CUMAT (1992), ${ }^{5}$ Morales $(1993,1996),{ }^{9}$ DAVIES (1993).

Anmerkungen: Unterschiede in den Flächenangaben für die bezeichneten Gebiete sind darauf zurückzuführen, dass jeweils nur unterschiedlich große Teilbereiche in diesen analysiert wurden. Zudem handelt es sich bei den Bezeichnungen ,zentrale Zone' und ,Expansionszone' um hypothetische Gebiete, denen keine klar definierte Flächengröße zugrunde liegt.

Die Landsat Szene 231/72 beinhaltet den größten Teil der zentralen Zone; die Szenen 230/72 und 230/73 decken einen Teil der zentralen Zone und den größten Teil der Expansionszone ab (Abb. 8).

Die Abholzungsraten von DAVIES (1993) und STEININGER et al. (2001a) beziehen sich auf die Gebietsfläche, alle anderen auf die Waldfläche. 
Im einzelnen werden in diesem Kapitel folgende Punkte einer regionalen Betrachtung unterzogen:

- Ausmaß der landwirtschaftlichen Expansion (quantitative Bestimmung der Abholzungsraten)

- Ableitung der Landnutzungssysteme (Kleinbauern, Mennoniten, Agroindustrie)

- Analyse von Änderung (change detection) und Intensität (Nutzungsdauer und -system) der Landnutzung

Die Ergebnisse der Landnutzungsanalyse bilden eine wesentliche räumliche Datenbasis der Bodendegradationsanalyse, mittels derer abschließend regionale Aussagen über die Veränderung chemischer und physikalischer Bodeneigenschaften als Folge der Konversion natürlicher Vegetation in Ackerland getroffen werden (Kap.5). Potenzielle Veränderungen von Bodeneigenschaften unter Weidenutzung werden in dieser Studie nicht näher quantifiziert.

\subsubsection{Multispektrale Klassifikation}

Für jedes Jahr wurde gemäß dem Ziel, Dauer, Änderung und Expansion der Landnutzung im Untersuchungszeitraum $\mathrm{zu}$ erfassen, eine pixelbasierte Landbedeckungsklassifikation durchgeführt. Die Klassen wurden allgemein in Anbau-, Weide-, Brache- und Siedlungsflächen sowie Savannen, Gewässer, natürliche Sandflächen und Primär- und Sekundärwälder unterteilt. Anbaukulturen und unterschiedliche Waldtypen wurden nicht gesondert klassifiziert, da dies im Rahmen der Untersuchung nicht von Relevanz war ${ }^{17}$. Alle Waldformationen wurden gemeinsam mit den Dornbuschformationen des Chaco in einer Klasse zusammengefasst.

Methodisch kam mit der Kombination von unüberwachter und überwachter Klassifikation ein hybrider Ansatz zur Anwendung. Die vorab angewendete unüberwachte Klassifikation liefert einen Überblick über den spektralen Informationsgehalt eines Satellitenbildes und erlaubt Rückschlüsse auf die Anzahl erfolgversprechend trennbarer Klassen bezüglich ihres spezifischen Reflexionsverhaltens. Ein Vorteil dieser Methode besteht darin, potenzielle Reflexionsklassen zu ermitteln, die allein durch eine visuelle Interpretation eines Satellitenbildes nicht erkannt werden. Die Aufnahmen wurden mit dem ISODATA-Clusteralgorithmus $^{18}$ vorab unüberwacht klassifiziert und die Ergebnisse auf mögliche Landbedeckungsklassen hin interpretiert. Waren diese eindeutig zuzuordnen, fanden sie Eingang in

\footnotetext{
${ }^{17}$ Die in der Fernerkundung zur Anwendung kommenden Standartmethoden (ISODATA, Maximum-Likelihood) werden an dieser Stelle nicht en detail diskutiert. Diesbezüglich sei auf einschlägige Fachliteratur der Fernerkundung, wie z.B. JENSEN (1996) und LILLESAND \& KIEFER (2000) verwiesen.

${ }^{18}$ Bei der ISODATA-Klassifikation werden alle Bildelemente (Pixel) über eine Clusteranalyse automatisch in Klassen vergleichbarer spektraler Eigenschaften unterteilt.
} 
die Bildung von Klassensignaturen der anschließenden überwachten Klassifikation, bei der alle Bildelemente (Pixel) auf ihre Ähnlichkeit mit den Klassensignaturen zuvor festgelegter Trainingsgebiete verglichen und klassifiziert werden. Die Ausweisung von Trainingsgebieten erfolgte auf Grundlage der Gebietskenntnis (ground check).

\section{Signaturanalyse}

Die Definition der Trainingsgebiete erfolgte unter Zuhilfenahme thematischer Karten (CORDECRUZ \& KfW 1995, DE VRIES 1995, MASRENA et al. 1999, MDSMA 1995) und der Referenzdaten aus den Geländebeobachtungen (ground truth). Für die Erfassung der gesamten spektralen Varianz innerhalb einer Landbedeckungsklasse wurde für jede Klasse ein Satz in sich homogener Trainingsgebiete erstellt, die im Anschluss an die Klassifikation wieder in der entsprechenden Oberklasse zusammengefasst wurden. Die farblichen und texturellen Eigenschaften der Aufnahmen aus dem Jahr 2001 dienten vor dem Hintergrund der Geländekenntnisse als Interpretationshilfe bei der Analyse der älteren Satellitenbildszenen. Die Trennbarkeit der spektralen Signaturen der Trainingsgebiete wurde nach der Jeffries-Matusita-Distanz ${ }^{19}$ überprüft. Der JM-Algorithmus berechnet einen Distanzwert der die Ähnlichkeit zweier Oberflächen bezüglich ihrer Reflexionseigenschaften ausdrückt. Bereits vor einer Klassifikation lassen sich darüber schlecht trennbare Signaturen ausschließen und potenzielle Fehlzuweisungen reduzieren. Anhand der Distanzmaße und der Ergebnisse wiederholter überwachter Testklassifikationen mit dem Maximum-LikelihoodAlgorithmus $^{20}$ wurden die Trainingsgebiete iterativ optimiert, bis jeweils ein für die abschließende überwachte Klassifikation akzeptabler Signaturdatensatz vorlag.

Die Genauigkeit, mit der die Zuweisung der Trainingsgebietspixel zu den Landbedeckungsklassen erfolgte, wurden anhand von Fehlermatrizen (confusion matrix) bewertet (Tab.A3, Anhang). Diese geben anhand verschiedener Maße einen Überblick, wie gut sich die gewählten Trainingsgebiete im spektralen Raum unterscheiden und deuten damit die Klassifikationsgenauigkeit im Ganzen an. Einschränkungen bestehen dahingehend, dass anhand der Fehlermatrizen kein Hinweis gegeben wird, ob die klassifizierten Pixel der Trainingsgebiete auch tatsächlich der beabsichtigten Landbedeckungs-klasse entsprechen. Eine diesbezügliche Kontrolle muss visuell durchgeführt werden. Naturgemäß treten bei der Klassifikation optischer Satellitendaten Fehler auf, die ihre Ursache in ähnlichen spektralen Reflexionen von Oberflächen haben. Dies äußert sich im Auftreten klassenfremder Pixel innerhalb homogener Areale sowie in der Überschneidung spektraler Signaturen verschiedener Objektklassen.

\footnotetext{
${ }^{19}$ Die Jeffries-Matusita-Distanzen variieren zwischen 0 und 2, wobei größere Werte eine bessere statistische Trennbarkeit der Signaturen bedeuten und ein genaueres Klassifikationsresultat erwarten lassen. Allgemein gilt: > 1,9: gute Trennbarkeit, 1,0 - 1,9 geringe Trennbarkeit, $<1,0$ schlechte Trennbarkeit (RICHARDS \& JIA 1999).

${ }^{20}$ Der Maximum-Likelihood-Algorithmus berechnet auf Basis statistischer Parameter der zuvor definierten Trainingsgebiete die Wahrscheinlichkeit, mit der ein Pixel des Satellitenbildes den Trainingsgebieten zugeordnet werden kann.
} 


\section{Ergebniskontrolle}

Die visuelle Kontrolle ergab, dass die Landbedeckungsklassen in der Mehrzahl korrekt erfasst wurden. Dennoch traten vereinzelt Fehlklassifikationen auf, die einer Nachbearbeitung bedurften. Diese bestanden in zum Teil analogen Reflexionseigenschaften zwischen den Landbedeckungsklassen, die sich mehrheitlich auf die Aufnahmezeitpunkte der Satellitenbilder in der Trockenzeit zurückführen lassen. Fehlzuweisungen wurden entsprechend der Zielsetzung nur dann als solche interpretiert, wenn diese zwischen zwei Landbedeckungsklassen auftraten, nicht aber zwischen zwei Trainingsgebieten innerhalb einer Klasse.

Die größten Überschneidungen und damit die häufigsten Fehlzuweisungen traten erwartungsgemäß zwischen Siedlungsflächen und vegetationsfreien Ackerböden respektive natürlichen Sandflächen auf. Dies ist wenig erstaunlich, da es sich bei Siedlungen um spektrale Mischklassen handelt, in denen diverse Oberflächen äquivalente Reflexionseigenschaften mit vegetationsfreien Oberflächen besitzen. Der Anteil vegetationsfreier Ackerflächen ist in der Trockenzeit generell hoch, da sowohl der Boden für die Einsaat der Sommerkulturen vorbereitet wird, als auch die Ernte der Winterkulturen erfolgt (Tab.A4, Anhang). Um Fehlzuweisungen zwischen diesen Klassen $\mathrm{zu}$ vermeiden, wurden die Siedlungsbereiche ausmaskiert und von der Klassifikation ausgeschlossen. Überschneidungen bestanden ferner zwischen Savannen und Bracheflächen. Bei beiden Klassen handelt es sich um spektrale Mischklassen mit unterschiedlichen Anteilen an Gehölzpflanzen und holzfreien Arten deren Reflexionseigenschaften sich zum Teil entsprechen. Gleiches galt für die zahlreichen brachgefallenen Anbauflächen der zentralen Zone, auf denen rezent extensive Weidewirtschaft betrieben wird. Diese Flächen wurden generell als Weide klassifiziert. Insofern bestanden bei dieser Klasse spektrale Analogien mit den extensiv beweideten, natürlichen Savannenflächen. Anbauflächen, auf denen sich nach der Ernte einjährige Gräser angesiedelt hatten, ähneln in ihrem Reflexionsverhalten Weiden und wurden partiell als solche klassifiziert. Demnach ist der reale Weideflächenanteil etwas geringer, als über die Klassifikationsergebnisse wiedergegeben. Im Süden des Untersuchungsgebiets wiesen Brachen im fortgeschrittenen Sukzessionsstadium und die natürliche Dornbuschvegetation des Chaco ähnliche Reflexionseigenschaften auf. Ebenso zeigten Primär- und Sekundärwälder z.T. ähnliche spektrale Signaturen, was aber im Rahmen der Fragestellung dieser Arbeit von untergeordneter Bedeutung war, da es sich nicht um Rodungsklassen handelt.

\section{Nachbearbeitung}

Die Fehlklassifikationen konnten mehrheitlich über die Nachbearbeitung mit Filtern korrigiert werden. Einzelpixel wurden mittels eines Majority-Filters ${ }^{21}$ (Fenstergröße $3 * 3$ )

\footnotetext{
${ }^{21}$ Innerhalb einer vorgegebenen Fenstergröße wird dem jeweiligen Zentralpixel der häufigste Klassenwert zugeordnet. Dabei wird die Annahme vorausgesetzt, dass der häufigste Klassenwert die dominante Landbedeckung repräsentiert.
} 
umgebungsbezogen reklassifiziert und zusammenhängende Pixelgruppen über die klassenspezifische Anwendung eines Sieve-Filter ${ }^{22}$ entfernt.

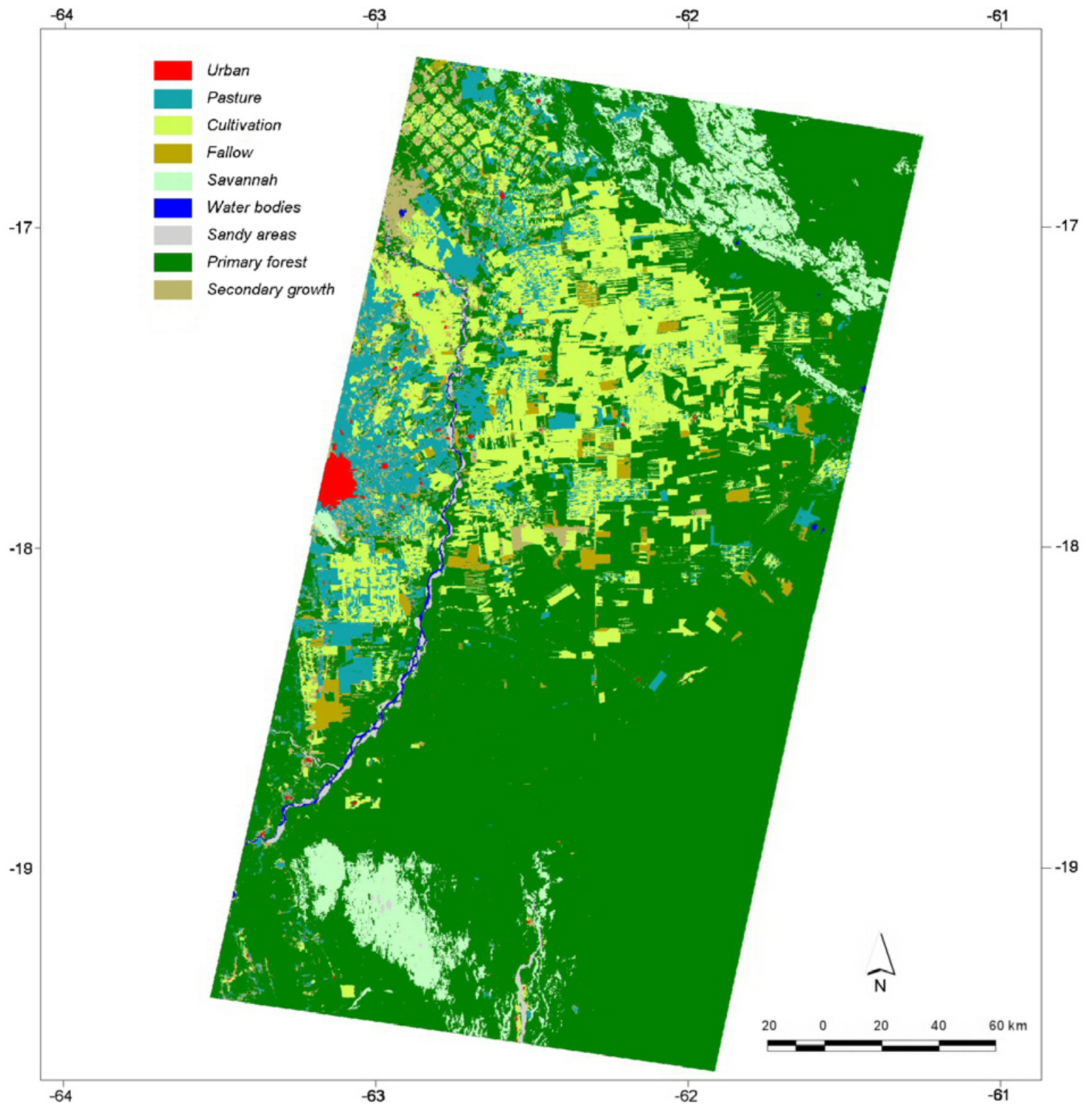

Abb. 11: Landnutzung und Vegetation 2001, basierend auf Klassifikation von Landsat 7-ETM+ Daten

Die verbliebenen Fehlzuweisungen zwischen vegetationsfreien Ackerböden und natürlichen Sandflächen wurden auf Grundlage ihrer spezifischen räumlichen Anordnung umkodiert. Bereiche hoher Reflexion innerhalb klar erkennbarer Feldgrenzen oder vergesellschaftet mit Straßen wurden in die Anbauklasse übernommen, wohingegen diese entlang von Flussläufen und den bekannten Dünenbereichen (Lomas de Arena, Arenales de Guanaco) als natürlich vorausgesetzt wurden. Gleiches Prinzip galt bei den noch vorhandenen Fehlinter-

\footnotetext{
${ }^{22}$ alle Pixelgruppen oder Einzelpixel die kleiner als ein definierter Wert sind, werden der umgebenden Klasse zugeordnet.
} 
pretationen zwischen Savannen und Bracheflächen, die aufgrund der markanten Objektgeometrie agroindustriell genutzter Felder problemlos umkodiert werden konnten (Abb.12).
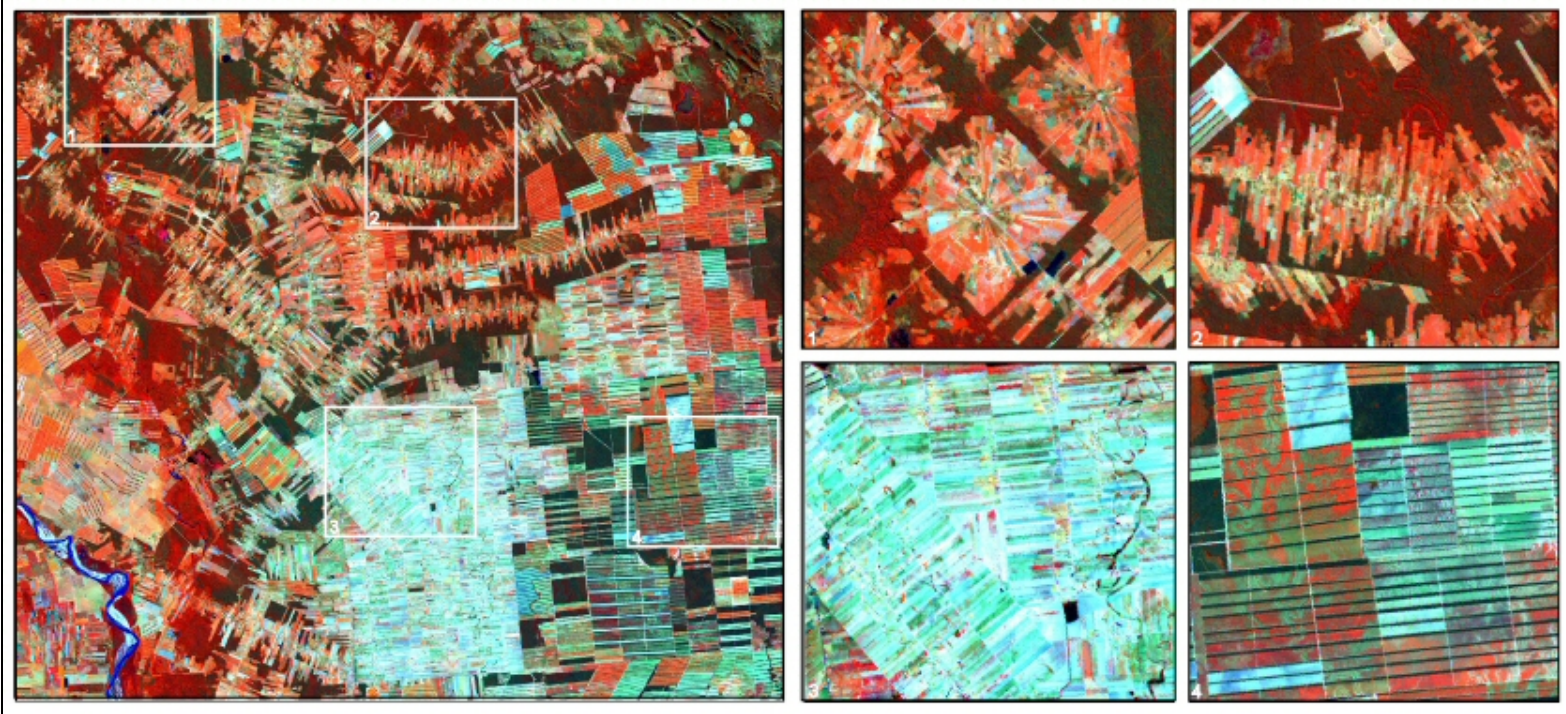

(1) Kleinbauern, geplante Kolonisation, (2) Kleinbauern, spontane Kolonisation, (3) Mennoniten, (4) Agroindustrie (Kanalkombination 4-5-3)

Abb. 12: Erscheinungsmuster der Landnutzungssysteme im Landsat-7 ETM+ Bild

Vermeintliche Savannenflächen innerhalb der bekannten Nutzflächen wurden der Bracheklasse zugewiesen. Umgekehrt wurden innerhalb der Savannenlandschaften als Brache klassifizierte Bereiche, sofern diese diffuse Objektgrenzen aufwiesen und sich nicht in der Nähe von Siedlungen befanden, in die Savannenklasse übernommen. Die auf den agroindustriellen Flächen der Expansionszone als Weide klassifizierten Bereiche, bei denen es sich vermeintlich um einjährige Gräser nach der Ernte handelt, wurden in die Anbauklasse übernommen. Bei diesen handelte es sich ausschließlich um von Windschutzstreifen eingefasste typische Anbauflächen der Expansionszone, die sich deutlich von den großflächigen Weidegebieten unterscheiden. Bei den anderen Nutzergruppen wurden keine wissensbasierten Änderungen vorgenommen, da alle zusätzlich Weidewirtschaft betreiben. In diesen liegt der klassifizierte Weideflächenanteil wegen ähnlicher spektraler Eigenschaften einjähriger Gräser und kultivierter Weidegräser stellenweise über dem realen.

Insgesamt zeigt sich, dass in der Trockenzeit einige Klassen über einen multispektralen Ansatz nicht immer eindeutig zu trennen sind. Gleichwohl konnte über die Nachbearbeitung eine homogene Flächendarstellung erreicht werden. Abschließend wurden alle Trainingsgebiete in der beabsichtigten Landbedeckungsklasse zusammengefasst und für jedes Jahr Flächenbilanzen berechnet. 


\subsubsection{Ableitung der Landnutzungssysteme}

Die Ableitung der verschiedenen Landnutzungssysteme erfolgte, basierend auf den Ergebnissen der multispektralen Klassifikation, mittels visueller Interpretation der Satellitenbilder unter Zuhilfenahme verschiedener Kartenwerke (COMITE CENTRAL MENONITA 1995, DE VRIES 1995, ENNS 1996) und Literaturquellen (MOHNHEIM \& KÖSTER 1982, SORIA MArTínez 1996, Thiele 1993b). Dabei wurde den Klassen "Siedlung", "Weide", "Anbau", "Brache" und "Sekundärwald" in der Datenbank ein Code der jeweiligen Produzentengruppe zugewiesen. Grundlage dieser Zuweisung war, dass die Gebiete der verschiedenen Produzenten in ihrem räumlichen Erscheinungsbild bestimmte Charakteristika aufweisen, die sich im Satellitenbild gut voneinander differenzieren lassen (Abb.12).

Den kleinbäuerlichen Kolonisationsgebieten ist ein kleinräumiges Mosaik von Nutzungsflächen, Sekundärvegetation und Waldfragmenten eigen, wie es bei keinem anderen System zu finden ist. Zudem liegt die durchschnittliche Größe der Produktionsflächen deutlich unter denen der anderen Systeme (Kap.3.2). Im NW des Untersuchungsgebiets gruppieren sich die Produktionsflächen in den dortigen geplanten Kolonien radial um einen zentralen Dorfkern, während spontane Kolonisationsgebiete durch die Anordnung meist rechteckiger Felder entlang von Straßen auffällig sind, das sogenannte Fischgrätenmuster (Abb.12)

Kennzeichen der agroindustriellen Anbauzonen sind kilometerlange, von breiten, linearen Windschutzstreifen eingefasste Produktionsflächen sowie das Fehlen von Siedlungsflächen. Auffällig ist zudem, wie auch in den Gebieten der Mennonitenkolonien, das Fehlen größerer Bereiche mit Sekundärvegetation (Abb.11). Mennoniten haben, durch die ihnen eigene Praxis, ihren Grundbesitz komplett zu entwalden, große zusammenhängende Areale von Produktionsflächen geschaffen. Da große Bereiche ihrer Gebiete zum Zeitpunkt der Aufnahmen von 2001 und 1984 ohne Vegetationsbedeckung waren, zeichnen sich diese durch hohe Reflexionswerte aus und sind eindeutig in den Satellitenbildern zu identifizieren (Abb.12). Die drei japanischen Kolonien (Okinawa 1-3) im Untersuchungsgebiet sind gleichermaßen über das Erscheinungsbild großer zusammenhängender Bereiche von Kulturland, allenfalls mit kleinen Windschutzstreifen, im Satellitenbild zu identifizieren. Hilfreich war zudem eine von FAN zur Verfügung gestellte Karte (o. Bez.), in der die Grenzen der Kolonien wiedergegeben sind.

\subsubsection{Entwicklung der Waldkonversion}

\section{Untersuchungsgebiet}

Zu Beginn der Untersuchungsperiode 1984 waren knapp $4.000 \mathrm{~km}^{2}$ Primärwald gerodet, der überwiegende Teil davon in der zentralen Zone um die Stadt Santa Cruz (Abb.13). In den folgenden Jahren schritt die Rodung jährlich um $459 \mathrm{~km}^{2}$ auf annähernd $5.900 \mathrm{~km}^{2}$ landwirtschaftliche Nutzfläche im Jahre 1992 voran, gleichbedeutend mit einer Abholzungsrate von 1,0 \% pro Jahr. Durch die starke landwirtschaftliche Expansion in den 1990er Jahren 
steigerte sich die Abholzung gegenüber den 1980er Jahren auf über $1.000 \mathrm{~km}^{2}$ pro Jahr, entsprechend einem jährlichen Waldflächenverlust von 2,8\%. Insgesamt wurden zwischen 1984-2001 $13.080 \mathrm{~km}^{2}$ Wald in eine landwirtschaftliche Nutzung und Siedlungsflächen überführt. Davon entfallen allein $9.405 \mathrm{~km}^{2}$ auf den Zeitraum von 1992-2001. Bezogen auf die Primärwaldfläche bedeutet das eine Reduktion um 25 \% in 17 Jahren (Tab.12, 13, Abb.13, 14).

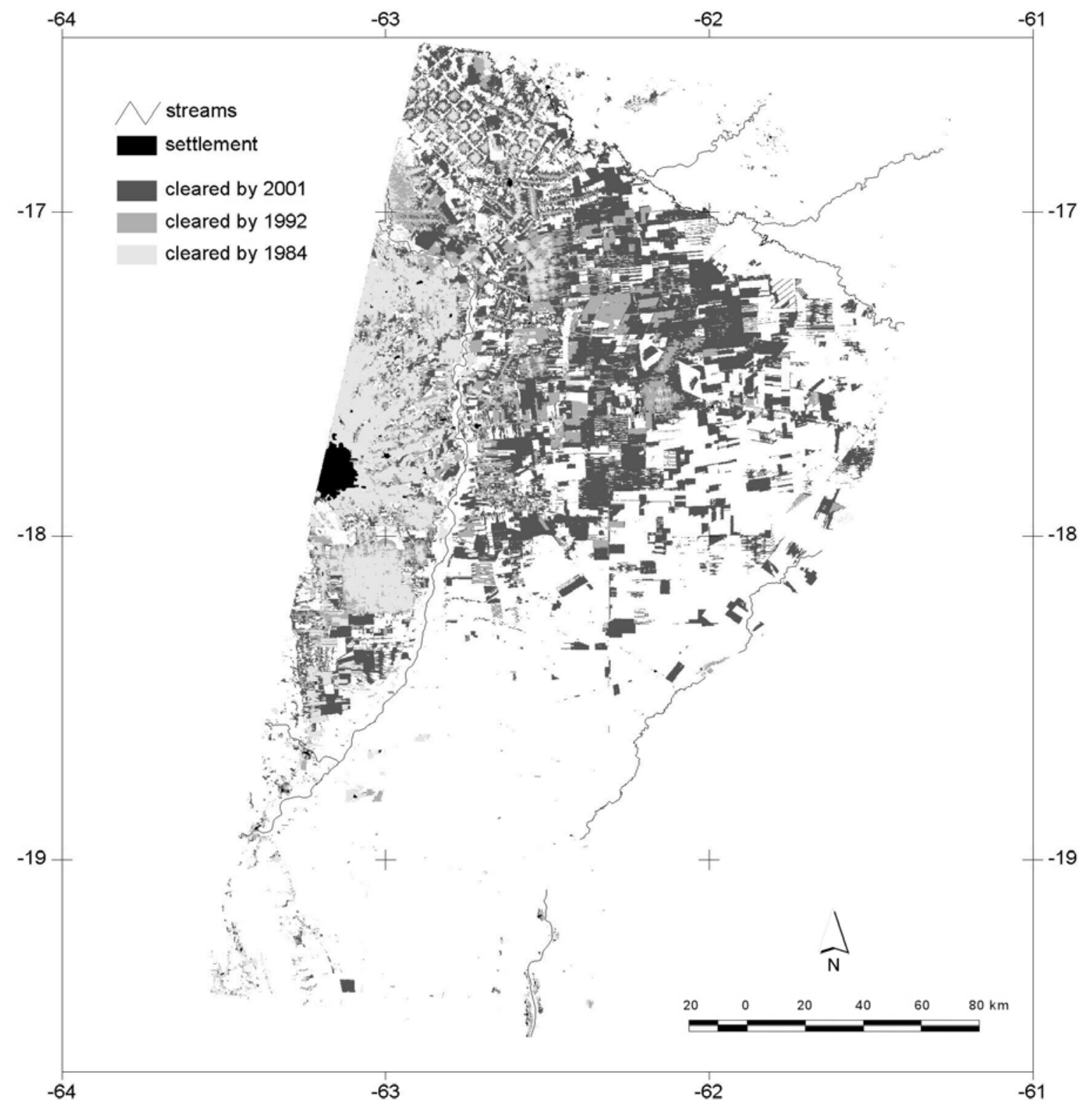

Abb. 13: Waldkonversion 1984-2001, basierend auf Klassifikation von Landsat 4, 5 TM und 7 ETM+ Daten

Der größte Anteil der Waldkonversion entfällt auf den Anbau landwirtschaftlicher Kulturen (Tab.3), als unmittelbare Folge der staatlichen Förderung einer industrialisierten Landwirtschaft (Kap.2.3.1). Im Untersuchungszeitraum hat sich die Anbaufläche von 1.869 $\mathrm{km}^{2}$ im Jahre 1984 auf $8.952 \mathrm{~km}^{2}$ im Jahre 2001 knapp verfünffacht. Mit 15,4 \% Anteil am 
Untersuchungsgebiet stellen die Anbauflächen im Jahr 2001 den weitaus höchsten Anteil der Nutzflächen. Auch bei den Weideflächen zeigt sich eine deutliche Expansion von $1.264 \mathrm{~km}^{2}$ im Jahr 1984 auf $4.166 \mathrm{~km}^{2}$ im Jahr 2001.

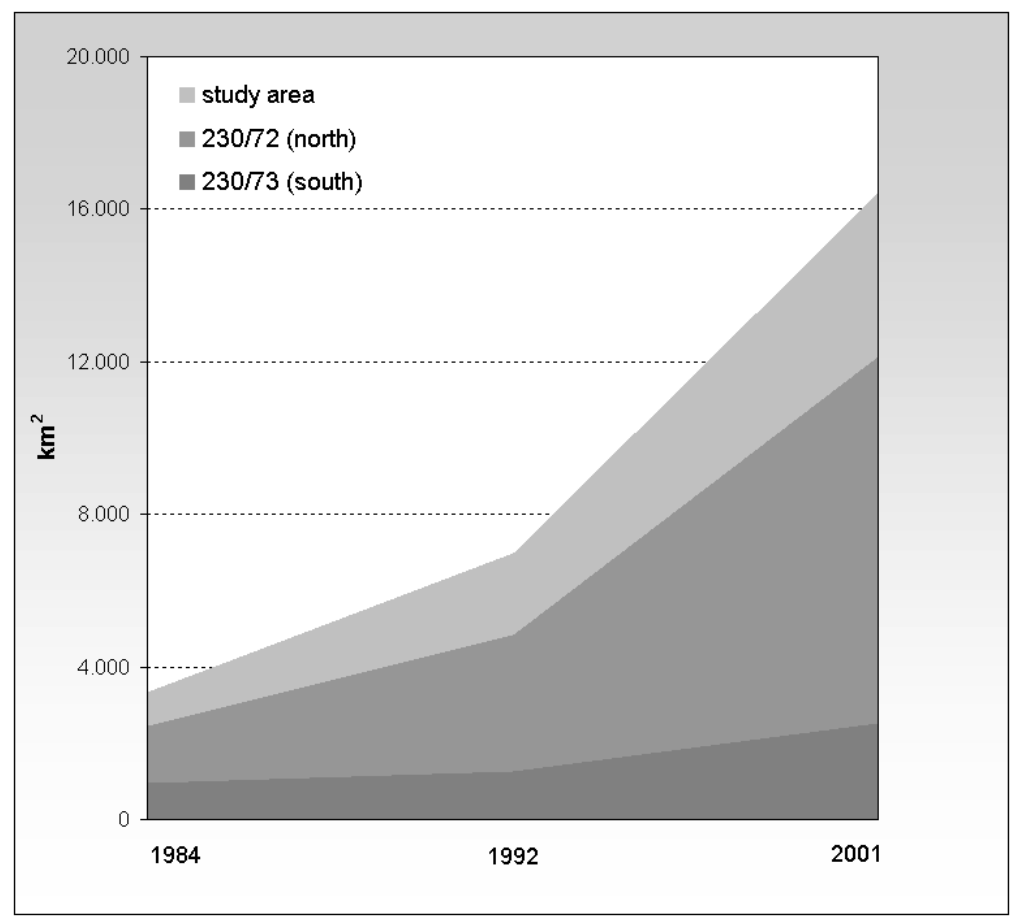

Abb. 14: Zunahme der Rodungsfläche im Untersuchungsgebiet 1984-2001

Im Vergleich mit 1984 entspricht das einem Flächenzuwachs von 230 \%. Bei einem Großteil der 2001 als Weide klassifizierten Flächen handelt es sich um brach gefallene, ehemalige Anbauflächen in der zentralen Zone, auf denen rezent extensive Weidenutzung praktiziert wird. Der Anteil der Bracheflächen ist bis 1992 konstant, zeigt aber 2001 einen signifikanten Anstieg auf $964 \mathrm{~km}^{2}$, gleichbedeutend mit einer Verachtfachung der Bracheflächen gegenüber 1992. Das begründet sich a priori durch die massive Zunahme der Anbauflächen bis 2001, kann aber gleichzeitig als Indiz der Ende der 1990er Jahre gesunkenen Weltmarktpreise für Soja und eines demzufolge weniger lukrativen Anbaus gedeutet werden. Der Anteil der Siedlungsflächen verdreifachte sich innerhalb der Untersuchungsperiode, nimmt aber 2001 mit $233 \mathrm{~km}^{2}$ zusammen nur 0,4\% am Untersuchungsgebiet ein. Von diesen entfallen allein $175 \mathrm{~km}^{2}$ auf die Stadt Santa Cruz (Tab.11, 12, 20).

\section{Nordhälfte (230/72)}

Im nördlichen Teil des Untersuchungsgebietes ist den günstigeren klimatischen Bedingungen zufolge eine deutlich stärkere landwirtschaftliche Aktivität zu verzeichnen. Von den $13.080 \mathrm{~km}^{2}$ Gesamtrodung im Untersuchungszeitraum entfallen allein $11.422 \mathrm{~km}^{2}$ auf die Region nördlich $18^{\circ}$ südl. Breite. 
Tab. 11: Flächenanteil der Landbedeckungsklassen (Untersuchungsgebiet)

\begin{tabular}{lrrrrrr}
\hline Klasse & \multicolumn{2}{c}{$\mathbf{1 9 8 4}$} & \multicolumn{2}{c}{$\mathbf{1 9 9 2}$} & \multicolumn{2}{c}{$\mathbf{2 0 0 1}$} \\
& $\mathbf{k m}^{\mathbf{2}}$ & {$[\mathbf{\%}]$} & $\mathbf{k m}^{\mathbf{2}}$ & {$[\mathbf{\%}]$} & $\mathbf{k m}^{\mathbf{2}}$ & {$[\mathbf{\%}]$} \\
\hline Siedlung & 71 & 0,1 & 128 & 0,2 & 233 & 0,4 \\
Weide & 1.264 & 2,2 & 2.039 & 3,5 & 4.166 & 7,2 \\
Anbau & 1.869 & 3,2 & 3.684 & 6,3 & 8.952 & 15,4 \\
Brache & 122 & 0,2 & 136 & 0,2 & 964 & 1,7 \\
Sekundärwald & 717 & 1,2 & 1.357 & 2,3 & 1.747 & 3,0 \\
Primärwald & 50.294 & 86,4 & 46.750 & 80,3 & 37.889 & 65,1 \\
Savanne & 3.176 & 5,5 & 3.395 & 5,8 & 3.689 & 6,3 \\
Sand & 594 & 1,0 & 416 & 0,7 & 380 & 0,7 \\
Gewässer & 90 & 0,2 & 154 & 0,3 & 177 & 0,3 \\
Wolken & 0 & 0,0 & 139 & 0,2 & 0 & 0,0 \\
\hline Gebiet & $\mathbf{5 8 . 1 9 7}$ & $\mathbf{1 0 0}$ & $\mathbf{5 8 . 1 9 7}$ & $\mathbf{1 0 0}$ & $\mathbf{5 8 . 1 9 7}$ & $\mathbf{1 0 0}$ \\
\hline
\end{tabular}

Tab. 12: Veränderung des Flächenanteils der Landbedeckungsklassen (Untersuchungsgebiet)

\begin{tabular}{lrrrrrr}
\hline Klasse & \multicolumn{2}{c}{$\mathbf{1 9 8 4} \mathbf{- 1 9 9 2}$} & \multicolumn{2}{c}{$\mathbf{1 9 9 2}-\mathbf{2 0 0 1}$} & \multicolumn{2}{c}{$\mathbf{1 9 8 4}-\mathbf{2 0 0 1}$} \\
& $\mathbf{k m}^{\mathbf{2}}$ & $*[\mathbf{\%}]$ & $\mathbf{k m}^{\mathbf{2}}$ & $*[\mathbf{\%}]$ & $\mathbf{k m}^{\mathbf{2}}$ & $*[\mathbf{\%}]$ \\
\hline Siedlung & 57 & 80 & 105 & 82 & 162 & 227 \\
Weide & 775 & 61 & 2.128 & 104 & 2.903 & 230 \\
Anbau & 1.815 & 97 & 5.268 & 143 & 7.083 & 379 \\
Brache & 13 & 11 & 828 & 611 & 842 & 689 \\
Sekundärwald & 640 & 89 & 390 & 29 & 1.030 & 144 \\
Primärwald & -3.545 & -7 & -8.861 & -19 & -12.405 & -25 \\
Savanne & 219 & 7 & 294 & 9 & 513 & 16 \\
Sand & -178 & -30 & -36 & -9 & -214 & -36 \\
Gewässer & 64 & 71 & 23 & 15 & 88 & 97 \\
Wolken & 139 & - & -139 & - & 0 & - \\
\hline
\end{tabular}

*Veränderung gegenüber Ausgangswert

Tab. 13: Entwaldungsrate (Untersuchungsgebiet )

\begin{tabular}{lccccrrrr}
\hline Periode & Gebiet & Primär- & \multicolumn{3}{c}{ Rodung } \\
& & wald & \multicolumn{2}{c}{ Periode } & \multicolumn{2}{c}{ pro Jahr } \\
& $\mathbf{k m}^{2}$ & $\mathbf{k m}^{\mathbf{2}}$ & $\mathbf{k m}^{\mathbf{2}}$ & {$[\%]$} & $*[\%]$ & $\mathbf{k m}^{\mathbf{2}}$ & $*[\%]$ & $* *[\%]$ \\
\hline bis 1984 & 58.197 & 50.294 & - & - & - & - & - & - \\
$\mathbf{1 9 8 4 - 1 9 9 2}$ & 58.197 & 46.750 & 3.674 & 28,1 & 6,3 & 459 & 0,8 & $-1,0$ \\
$\mathbf{1 9 9 2 - 2 0 0 1}$ & 58.197 & 37.889 & 9.405 & 71,9 & 16,2 & 1.045 & 1,8 & $-2,8$ \\
\hline $\mathbf{1 9 8 4 - 2 0 0 1}$ & 58.197 & 37.889 & 13.080 & 100 & 22,5 & 769 & 1,3 & $-2,0$ \\
\hline
\end{tabular}

*Flächenanteil im Untersuchungsgebiet, **Flächenanteil an Waldfläche 
Tab. 14: Flächenanteil der Landbedeckungsklassen (nördliches Untersuchungsgebiet, WRS-2 230/72)

\begin{tabular}{lrrrrrr}
\hline Klasse & \multicolumn{2}{c}{$\mathbf{1 9 8 4}$} & \multicolumn{2}{c}{$\mathbf{1 9 9 2}$} & \multicolumn{2}{c}{$\mathbf{2 0 0 1}$} \\
& $\mathbf{k m}^{\mathbf{2}}$ & {$[\mathbf{\%}]$} & $\mathbf{k m}^{\mathbf{2}}$ & {$[\mathbf{\%}]$} & $\mathbf{k m}^{\mathbf{2}}$ & {$[\mathbf{\%}]$} \\
\hline Siedlung & 62 & 0,2 & 119 & 0,4 & 214 & 0,7 \\
Weide & 990 & 3,3 & 1.562 & 5,2 & 3.356 & 11,3 \\
Anbau & 1.312 & 4,4 & 3.101 & 10,4 & 7.867 & 26,4 \\
Brache & 79 & 0,3 & 51 & 0,2 & 681 & 2,3 \\
Sekundärwald & 648 & 2,2 & 1.109 & 3,7 & 1.432 & 4,8 \\
Primärwald & 24.800 & 83,2 & 21.513 & 72,2 & 13.970 & 46,9 \\
Savanne & 1.701 & 5,7 & 2.117 & 7,1 & 2.109 & 7,1 \\
Sand & 178 & 0,6 & 159 & 0,5 & 113 & 0,4 \\
Gewässer & 42 & 0,1 & 81 & 0,3 & 70 & 0,2 \\
Wolken & 0 & 0,0 & 0 & 0,0 & 0 & 0,0 \\
\hline Gebiet & $\mathbf{2 9 . 8 1 3}$ & $\mathbf{1 0 0}$ & $\mathbf{2 9 . 8 1 3}$ & $\mathbf{1 0 0}$ & $\mathbf{2 9 . 8 1 3}$ & $\mathbf{1 0 0}$ \\
\hline
\end{tabular}

Tab. 15: Veränderung des Flächenanteils der Landbedeckungsklassen (nördliches Untersuchungsgebiet, WRS-2 230/72)

\begin{tabular}{|c|c|c|c|c|c|c|}
\hline \multirow[t]{2}{*}{ Klasse } & \multicolumn{2}{|c|}{1984 - 1992} & \multicolumn{2}{|c|}{$1992-2001$} & \multicolumn{2}{|c|}{$1984-2001$} \\
\hline & $\mathbf{k m}^{2}$ & $*[\%]$ & $\mathbf{k m}^{2}$ & $*[\%]$ & $\mathbf{k m}^{2}$ & $*[\%]$ \\
\hline Siedlung & 56 & 90 & 95 & 80 & 151 & 242 \\
\hline Weide & 572 & 58 & 1.793 & 115 & 2.366 & 239 \\
\hline Anbau & 1.789 & 136 & 4.766 & 154 & 6.555 & 500 \\
\hline Brache & -27 & -35 & 629 & 1226 & 602 & 763 \\
\hline Sekundärwald & 461 & 71 & 323 & 29 & 784 & 121 \\
\hline Primärwald & -3.287 & -13 & -7.542 & -35 & -10.829 & -44 \\
\hline Savanne & 416 & 24 & -8 & 0 & 409 & 24 \\
\hline Sand & -19 & -11 & -46 & -29 & -65 & -36 \\
\hline Gewässer & 39 & 93 & -11 & -14 & 28 & 66 \\
\hline Wolken & - & - & - & - & - & - \\
\hline
\end{tabular}

*Veränderung gegenüber Ausgangswert

Tab. 16: Entwaldungsrate (nördliches Untersuchungsgebiet, WRS-2 230-72)

\begin{tabular}{|c|c|c|c|c|c|c|c|c|}
\hline \multirow[t]{3}{*}{ Periode } & \multirow{3}{*}{$\begin{array}{c}\text { Gebiet } \\
\mathbf{k m}^{2} \\
\end{array}$} & \multirow{3}{*}{$\begin{array}{c}\text { Primär- } \\
\text { wald } \\
\mathbf{k m}^{2}\end{array}$} & \multicolumn{6}{|c|}{ Rodung } \\
\hline & & & \multicolumn{3}{|c|}{ Periode } & \multicolumn{3}{|c|}{ pro Jahr } \\
\hline & & & $\mathbf{k m}^{2}$ & [\%] & ${ }^{*}[\%]$ & $\mathbf{k m}^{2}$ & ${ }^{*}[\%]$ & *[\%] \\
\hline bis 1984 & 29.813 & 24.800 & - & - & - & - & - & - \\
\hline 1984-1992 & 29.813 & 21.513 & 3.210 & 28,1 & 10,8 & 401 & 1,3 & $-1,9$ \\
\hline 1992-2001 & 29.813 & 13.970 & 8.211 & 71,9 & 27,5 & 912 & 3,1 & $-6,5$ \\
\hline 1984-2001 & 29.813 & 13.970 & 11.422 & 100 & 38,3 & 672 & 2,3 & $-4,8$ \\
\hline
\end{tabular}

*Flächenanteil im Untersuchungsgebiet, **Flächenanteil an Waldfläche 
Tab. 17: Flächenanteil der Landbedeckungsklassen (südliches Untersuchungsgebiet, WRS-2 230/73)

\begin{tabular}{lrrrrrr}
\hline Klasse & \multicolumn{2}{c}{$\mathbf{1 9 8 4}$} & \multicolumn{2}{c}{$\mathbf{1 9 9 2}$} & \multicolumn{2}{c}{$\mathbf{2 0 0 1}$} \\
& $\mathbf{k m}^{\mathbf{2}}$ & {$[\mathbf{\%}]$} & $\mathbf{k m}^{\mathbf{2}}$ & {$[\mathbf{\%}]$} & $\mathbf{k m}^{\mathbf{2}}$ & {$[\mathbf{\%}]$} \\
\hline Siedlung & 9 & 0,0 & 9 & 0,0 & 19 & 0,1 \\
Weide & 303 & 1,0 & 525 & 1,8 & 890 & 3,0 \\
Anbau & 600 & 2,0 & 643 & 2,2 & 1.249 & 4,2 \\
Brache & 45 & 0,2 & 93 & 0,3 & 378 & 1,3 \\
Sekundärwald & 86 & 0,3 & 290 & 1,0 & 384 & 1,3 \\
Primärwald & 26.623 & 89,7 & 26.305 & 88,7 & 24.729 & 83,4 \\
Savanne & 1.521 & 5,1 & 1.306 & 4,4 & 1.626 & 5,5 \\
Sand & 431 & 1,5 & 279 & 0,9 & 281 & 0,9 \\
Gewässer & 50 & 0,2 & 76 & 0,3 & 111 & 0,4 \\
Wolken & 0 & 0,0 & 139 & 0,5 & 0 & 0,0 \\
\hline Gebiet & $\mathbf{2 9 . 6 6 7}$ & $\mathbf{1 0 0}$ & $\mathbf{2 9 . 6 6 7}$ & $\mathbf{1 0 0}$ & $\mathbf{2 9 . 6 6 7}$ & $\mathbf{1 0 0}$ \\
\hline
\end{tabular}

Tab. 18: Veränderung des Flächenanteils der Landbedeckungsklassen (südliches Untersuchungsgebiet, WRS-2 230/73)

\begin{tabular}{lrrrrrr}
\hline Klasse & \multicolumn{2}{c}{$\mathbf{1 9 8 4} \mathbf{- 1 9 9 2}$} & \multicolumn{2}{c}{$\mathbf{1 9 9 2} \mathbf{- 2 0 0 1}$} & \multicolumn{2}{c}{$\mathbf{1 9 8 4}-\mathbf{2 0 0 1}$} \\
& $\mathbf{k m}^{\mathbf{2}}$ & $*[\mathbf{\%}]$ & $\mathbf{k m}^{\mathbf{2}}$ & $*[\mathbf{\%}]$ & $\mathbf{k m}^{\mathbf{2}}$ & $*[\mathbf{\%}]$ \\
\hline Siedlung & 1 & 7 & 10 & 105 & 10 & 119 \\
Weide & 223 & 74 & 365 & 69 & 587 & 194 \\
Anbau & 43 & 7 & 606 & 94 & 649 & 108 \\
Brache & 48 & 107 & 285 & 305 & 333 & 738 \\
Sekundärwald & 204 & 238 & 94 & 32 & 298 & 347 \\
Primärwald & -319 & -1 & -1.576 & -6 & -1.895 & -7 \\
Savanne & -214 & -14 & 319 & 24 & 105 & 7 \\
Sand & -152 & -35 & 2 & 1 & -150 & -35 \\
Gewässer & 27 & 53 & 35 & 46 & 62 & 124 \\
Wolken & - & - & -139 & - & - & - \\
\hline
\end{tabular}

*Veränderung gegenüber Ausgangswert

Tab. 19: Entwaldungsrate (südliches Untersuchungsgebiet, WRS-2 230-73)

\begin{tabular}{lccrrrrrr}
\hline Periode & Gebiet & \multicolumn{2}{c}{ Primär- } & \multicolumn{3}{c}{ Rodung } \\
& & wald & \multicolumn{2}{c}{ Periode } & \multicolumn{3}{c}{ pro Jahr } \\
& $\mathbf{k m}^{2}$ & $\mathbf{k m}^{\mathbf{2}}$ & $\mathbf{k m}^{2}$ & {$[\%]$} & $*[\%]$ & $\mathbf{k m}^{\mathbf{2}}$ & $*[\%]$ & $* *[\%]$ \\
\hline bis 1984 & 29.667 & 26.623 & - & - & - & - & - & - \\
$\mathbf{1 9 8 4 - 1 9 9 2}$ & 29.667 & 26.305 & 527 & 26,1 & 1,8 & 66 & 0,2 & $-0,3$ \\
$\mathbf{1 9 9 2 - 2 0 0 1}$ & 29.667 & 24.729 & 1.491 & 73,9 & 5,0 & 166 & 0,6 & $-0,7$ \\
\hline $\mathbf{1 9 8 4 - 2 0 0 1}$ & 29.667 & 24.729 & 2.018 & 100 & 6,8 & 119 & 0,4 & $-0,5$ \\
\hline
\end{tabular}

*Flächenanteil im Untersuchungsgebiet, **Flächenanteil an Waldfläche 
Bis Mitte der 1980er Jahre beschränkte sich der Großteil der Rodungen auf die zentrale Zone. Östlich des Río Grande finden sich größere Rodungsgebiete lediglich in den neugegründeten Mennonitenkolonien sowie in der nordwestlichen Kleinbauernzone um San Julian. Zu diesem Zeitpunkt waren erst $10 \%$ der Gebietsfläche von Rodungen betroffen.

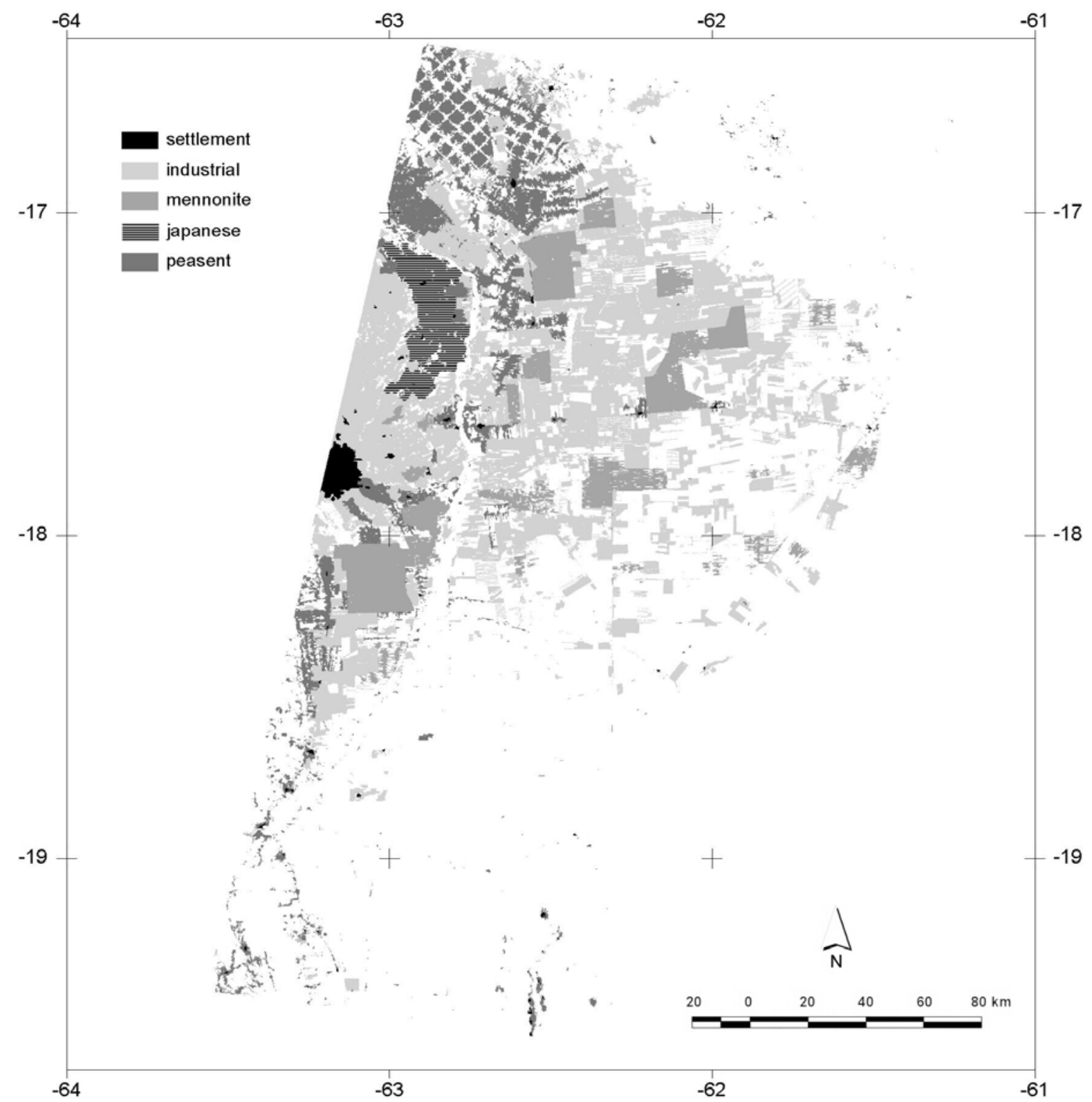

Abb. 15: Verteilung der Landnutzungssysteme 2001

Die Abholzungsrate lag bis Anfang der 1990er Jahre bei 1,9\% $\left(401 \mathrm{~km}^{2}\right)$ pro Jahr und stieg in den Folgejahren mit der landwirtschaftlichen Erschließung der Expansionszone ${ }^{23}$ auf 6,5 \% an (Kap.2.3.1). Bezogen auf die Gebietsfläche entspricht das einem jährlichen Zuwachs von $912 \mathrm{~km}^{2}$. In einer vergleichbaren Größenordnung liegen die Ergebnisse von CAMACHO et al. (2001), die zwischen 1993 und 2000, bei kleinerer Gebietsgröße, eine jährliche Abholzung

\footnotetext{
${ }^{23}$ Die Expansionszone ist zum größten Teil über die Landsat WRS-2 Szene 230-72 abgedeckt (Abb. 8).
} 
von $718 \mathrm{~km}^{2}$ konstatieren. Die sehr hohen Abholzungsraten in der Expansionszone spiegeln sich in einem kontinuierlichen Anstieg der Anbauflächen in nur neun Jahren um $4.766 \mathrm{~km}^{2}$ auf $7.867 \mathrm{~km}^{2}$ im Jahre 2001 wieder. 75 \% der gesamten Rodung gehen auf landwirtschaftliche Aktivitäten in den 1990er Jahren zurück. Insgesamt hat sich die Waldfläche in der Nordhälfte im Vergleich mit 1984 um fast die Hälfte verringert. Die mittlere Rodungsrate ist mit $672 \mathrm{~km}^{2}$ pro Jahr vergleichbar mit denen von BouNOUA et al. (2003) und STEININGER et al. (2001b), die einen jährlichen Waldflächenrückgang von $590 \mathrm{~km}^{2}$ (1986-1999) respektive 670 km² (1984-1998), bei gleichfalls unterschiedlichen Gebietsgrößen berechnen (Tab.10, 14, $15,16)$.

Tab. 20: Änderung der Klassenanteile an der Landnutzungsfläche 1984-2001 in [\%]

\begin{tabular}{lccccccccc}
\hline & \multicolumn{3}{c}{ Untersuchungsgebiet } & \multicolumn{3}{c}{ WRS-2 230/72 (Nord) } & \multicolumn{3}{c}{ WRS-2 230/73 (Süd) } \\
& 1984 & 1992 & 2001 & 1984 & 1992 & 2001 & 1984 & 1992 & 2001 \\
\hline Siedlung & 2,1 & 2,1 & 1,6 & 2,6 & 2,5 & 1,8 & 0,9 & 0,7 & 0,8 \\
Weide & 38,0 & 34,1 & 29,1 & 40,5 & 32,3 & 27,7 & 31,6 & 41,3 & 35,1 \\
Anbaufläche & 56,2 & 61,5 & 62,5 & 53,7 & 64,2 & 64,9 & 62,7 & 50,6 & 49,2 \\
Brache & 3,7 & 2,3 & 6,7 & 3,2 & 1,1 & 5,6 & 4,7 & 7,3 & 14,9 \\
\hline
\end{tabular}

Tab. 21: Flächenanteile der Produzentengruppen an der Landnutzungsfläche 1984-2001

\begin{tabular}{lrrrrrr}
\hline & \multicolumn{2}{c}{$\mathbf{1 9 8 4}$} & \multicolumn{2}{c}{1992} & \multicolumn{2}{c}{$\mathbf{2 0 0 1}$} \\
& $\mathrm{km}^{2}$ & {$[\%]$} & $\mathrm{km}^{2}$ & {$[\%]$} & $\mathrm{km}^{2}$ & {$[\%]$} \\
\hline Agroindustrie & 1.972 & 48,8 & 3.665 & 49,9 & 9.493 & 59,1 \\
Mennoniten & 803 & 19,9 & 1.315 & 17,9 & 2.679 & 16,7 \\
Japaner & 405 & 10,0 & 535 & 7,3 & 628 & 3,9 \\
Kleinbauern & 792 & 19,6 & 1.699 & 23,1 & 3.029 & 18,9 \\
Siedlung & 71 & 1,8 & 128 & 1,7 & 233 & 1,5 \\
Summe & $\mathbf{4 . 0 4 3}$ & $\mathbf{1 0 0}$ & $\mathbf{7 . 3 4 2}$ & $\mathbf{1 0 0}$ & $\mathbf{1 6 . 0 6 2}$ & $\mathbf{1 0 0}$ \\
\hline
\end{tabular}

Südhälfte (230/73)

Im Vergleich zum Norden ist der Anstieg der Abholzungsrate im trockeneren Süden ${ }^{24}$ von jährlich 0,3\%(66 km²) in den 1980er Jahren auf 0,7\%(166 $\left.\mathrm{km}^{2}\right)$ in den 1990er Jahren moderat und befindet sich im Mittel nur leicht über dem bolivianischen Landesdurchschnitt von $0,3 \%$ (Tab.19). In der Südhälfte konzentrierten sich die landwirtschaftlichen Nutzflächen bis Mitte der 1990er Jahre, aufgrund der höheren Niederschlagssummen, fast ausschließlich in der Nähe zum Andenrand. Erst 2001 zeigte sich in der südöstlichen Alluvialebene eine sukzessive Ausdehnung der agroindustriellen Nutzflächen über die imaginäre Grenze des 18. Breitengrades hinaus (Abb.13). Von 1984 bis 2001 wurden $2.018 \mathrm{~km}^{2}$ gerodet, davon gehen $74 \%$ auf die landwirtschaftliche Expansion in den 1990er Jahren zurück. Auch in der

\footnotetext{
${ }^{24}$ Beim direkten Vergleich der beiden Landsat-Szenen müssen die Zahlen als relative Größenordnungen betrachtet werden, da sich zwischen den Aufnahmen ein Überlappungsbereich von $1.283 \mathrm{~km}^{2}$ ergibt.
} 
Südhälfte nehmen die Anbauflächen den prozentual höchsten Anteil an der Nutzung ein, wenngleich bis 2001 eine leicht rückläufige Tendenz bei gleichzeitiger Steigerung des Anteils der Weide- und Bracheflächen zu erkennen ist. Dennoch entfiel 2001 noch immer die Hälfte der Nutzungsfläche auf den Anbau von Kulturen. Insgesamt ist der Süden, infolge der für eine landwirtschaftliche Nutzung weniger günstigen klimatischen Bedingungen, gegenüber dem Norden deutlich geringer anthropogen beeinflusst. Diese Tatsache dokumentiert sich über die Primärwaldfläche, die in der Untersuchungsperiode lediglich um $7 \%$ zurückging und 2001 noch einen Flächenanteil von 83 \% einnahm (Tab.17, 18, 19, 20).

\subsubsection{Anteil der Produzentengruppen an der Waldkonversion}

Wenngleich die Flächeninanspruchnahme durch landwirtschaftliche Aktivitäten Mitte der 1980er Jahre mit ca. $7 \%$ noch vergleichsweise gering ist, entfiel bereits die Hälfte aller landwirtschaftlichen Nutzflächen auf den agroindustriellen Sektor $\left(1.972 \mathrm{~km}^{2}\right)$. Diese gehen in der Mehrzahl auf den Baumwoll- und beginnenden Sojaanbau sowie größere Viehzuchtbetriebe in der zentralen Zone zurück. Nach Süden bestimmen zunehmend die Landbesitze der Mennoniten das Bild, die Ende der 1960er Jahre südöstlich von Santa Cruz mehrere große Kolonien gründeten. Bis 1984 gehen die höchsten Rodungsraten in dieser Zone auf die Mennoniten zurück, demgegenüber sie im gesamten Untersuchungsgebiet ein Fünftel aller landwirtschaftlichen Nutzflächen einnehmen $\left(803 \mathrm{~km}^{2}\right)$. In einer vergleichbaren Größenordnung liegen die Flächenanteile in den Kolonisationsgebieten der Kleinbauern $\left(792 \mathrm{~km}^{2}\right)$, während auf die japanischen Kolonien 1984 noch $10 \%\left(405 \mathrm{~km}^{2}\right)$ der Agrarfläche entfällt (Tab.21, Abb.15, 17).

Tab. 22: Flächenanteile der Produzentengruppen an der Rodung 1984-2001

\begin{tabular}{|c|c|c|c|c|c|c|c|c|c|}
\hline & \multicolumn{3}{|c|}{ 1984-1992 } & \multicolumn{3}{|c|}{ 1992-2001 } & \multicolumn{3}{|c|}{ 1984-2001 } \\
\hline & $\mathrm{km}^{2}$ & $*[\%]$ & $* *[\%]$ & $\mathrm{km}^{2}$ & $*[\%]$ & $* *[\%]$ & $\mathrm{km}^{2}$ & $*[\%]$ & $* *[\%]$ \\
\hline Agroindustrie & 1.944 & 52,9 & 99 & 6.034 & 64,2 & 165 & 7.978 & 61,0 & 405 \\
\hline Mennoniten & 575 & 15,7 & 72 & 1.385 & 14,7 & 105 & 1.960 & 15,0 & 244 \\
\hline Japaner & 201 & 5,5 & 50 & 168 & 1,8 & 31 & 369 & 2,8 & 91 \\
\hline Kleinbauern & 939 & 25,6 & 119 & 1.797 & 19,1 & 106 & 2.737 & 20,9 & 346 \\
\hline Siedlung & 15 & 0,4 & 21 & 21 & 0,2 & 17 & 36 & 0,3 & 51 \\
\hline gesamt & 3.674 & 100 & 91 & 9.405 & 100 & 128 & 13.080 & 100 & 324 \\
\hline
\end{tabular}

*Anteil an Rodung, **Veränderung (Expansion) gegenüber Ausgangswert

Zwischen 1984 und 1992 wurden über $3.600 \mathrm{~km}^{2}$ Wald für landwirtschaftliche Zwecke gerodet. Dabei ist der mit 53 \% höchste Anteil agroindustriellen Betrieben zuzuordnen, die ihre landwirtschaftlichen Nutzflächen von 1984 bis 1992 verdoppelt haben. Dies war vor allem eine Folge beginnender großflächiger Rodungen in der Expansionszone. Ein deutlicher 
Flächenzuwachs ist bis 1992 bei den Kleinbauern zu beobachten, die ihren Flächenanteil gegenüber 1984 mehr als verdoppelt haben und zu einem Viertel zur Waldkonversion beitrugen. Während Rodungen bis Mitte der 1980er Jahre hauptsächlich auf die geplanten Kolonisations-gebiete beschränkt waren, bestimmt mit Beginn der 1990er Jahre vermehrt spontane Kolonisation das Rodungsbild dieser Gruppe. Knapp 16 \% der Rodungen bis 1992 gehen auf die Expansion der Mennonitenkolonien zurück. Dabei zeigen sich großflächige Rodungen vor allem in den neuen Kolonien östlich des Río Grande (Kap.2.3.1). In den japanischen Kolonien ist die Expansion der Landwirtschaftsflächen mit $201 \mathrm{~km}^{2}$, entsprechend 5,5\% der Gesamtrodungsfläche, vergleichsweise gering. Der Grund ist darin zu sehen, dass nur innerhalb der Grenzen der 3 bestehenden Kolonien neue Flächen urbar gemacht wurden, nicht jedoch, wie bei den anderen Produzentengruppen, neue Gebiete erschlossen wurden (Tab.21, 22, Abb.16, 17).

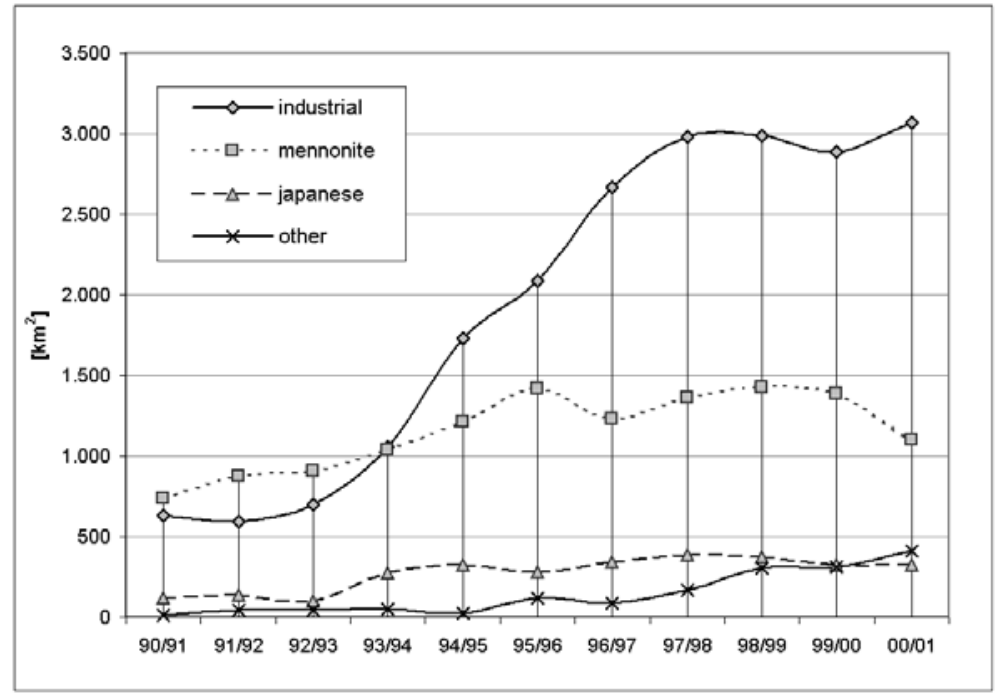

Quelle: CAO (2002)

Abb. 16: Entwicklung der Produktionsfläche für Sommersoja in den 1990er Jahren nach Produzenten

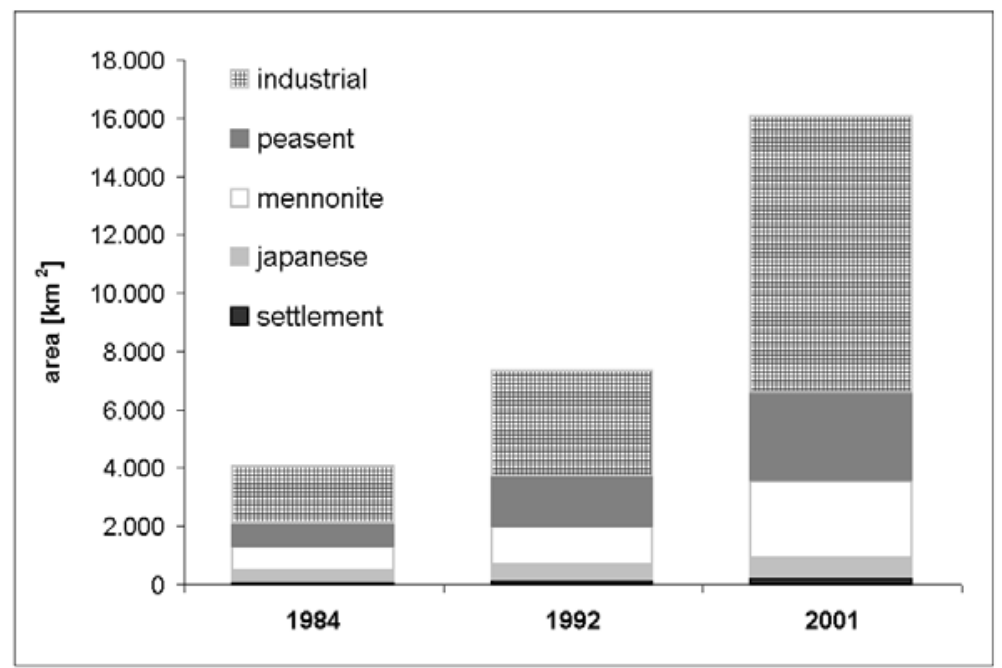

Abb. 17: Expansion landwirtschaftlicher Nutzflächen nach Produzenten 1984-2001 
In den 1990er Jahren (1992-2001) verzeichnet der agroindustrielle Sektor, v.a. als Folge des südamerikanischen Soja-Booms, enorme Steigerungsraten (Abb.16, Abb.17). Innerhalb von nur neun Jahren sind mehr als $6.000 \mathrm{~km}^{2}$ Rodungsfläche dieser Gruppe zuzurechnen, das entspricht zwei Drittel der Gesamtrodung von 1992-2001. Die Flächenausweitung in diesem Zeitraum ist fast ausschließlich auf die landwirtschaftliche Erschließung der Expansionszone, zurückzuführen. Bedingt durch die hohen Rodungsraten der Agroindustrie verschiebt sich das Anteilsverhältnis an der Rodung gegenüber 1992 insofern, dass sowohl Mennoniten, als auch Kleinbauern in geringerem Umfang an der Waldkonversion partizipieren. Gleichwohl verdoppeln beide ihre landwirtschaftlichen Nutzflächen und tragen mit $34 \% \mathrm{zu}$ ungefähr gleichen Anteilen zur Gesamtrodung bei. Die leicht rückläufige Tendenz der Flächenexpansion bei den Kleinbauern kann als Folge des seit Mitte der 1990er Jahre nachlassenden Migrationsstroms dieser Gruppe in den Agrarsektor interpretiert werden (Kap.2.3.1). Der geringste Flächenzuwachs ist in den japanischen Kolonien zu verzeichnen, die ihre Nutzfläche um $168 \mathrm{~km}^{2}$ erweitern und damit knapp $2 \%$ Anteil an der Rodung in diesem Zeitraum haben. Offensichtlich haben sie in den 1990er Jahren die Grenzen ihrer Kolonien erreicht (Tab.21, 22, Abb.17).

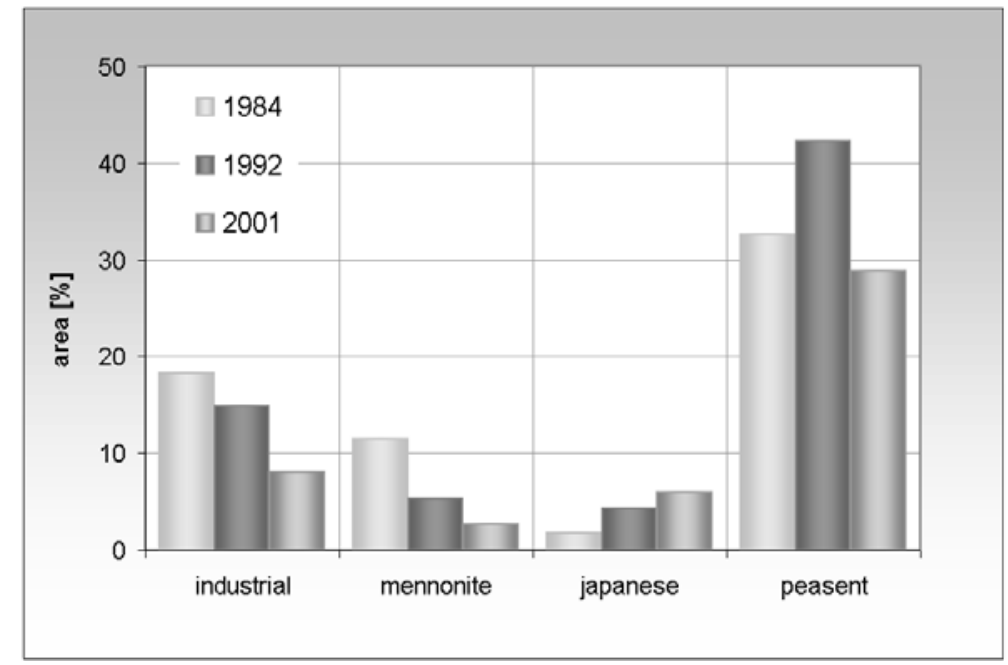

Abb. 18: Anteil an Sekundärwäldern in den Gebieten der verschiedenen Produzenten

Über den gesamten Untersuchungszeitraum sind die höchsten Rodungsraten der agroindustriellen Expansion zuzuordnen. Agroindustrielle Betriebe haben ihre landwirtschaftlichen Nutzflächen in den 17 Jahren auf $9.493 \mathrm{~km}^{2}$ verfünffacht. $61 \%$ aller Rodungen gehen auf diese Produzentengruppe zurück. Kleinbauern und Mennoniten tragen mit $21 \%$ respektive $15 \%$ in vergleichbarem Maße zur Waldkonversion bei. Gegenüber 1984 haben beide Produzentengruppen ihre Flächenanteile im Untersuchungsgebiet auf $3.029 \mathrm{~km}^{2}$ bzw. $2.679 \mathrm{~km}^{2}$ mehr als verdreifacht. Auffällig in den Gebieten der Kleinbauern ist ein hoher Anteil an jungen Sekundärwäldern (Abb.18). Dies begründet sich einerseits durch die Natur des slash and burn Systems mit Sekundärwäldern als Brachestadium, ist aber auch auf 
periodische Überschwemmungen zurückzuführen, in deren Folge immer wieder tausende von Hektar Kulturflächen verloren gehen. Davon besonders betroffen sind die Gebiete der Kleinbauern im Nordwesten des Untersuchungsgebietes (WACHHOLTZ \& HEROLD-MERGL 2003). Bedingt durch den hohen Sekundärwaldanteil ist der Anteil landwirtschaftlich genutzter Fläche der Kleinbauern gegenüber dem der Mennoniten zu allen drei Aufnahmezeitpunkten geringer. Die Mennoniten zeigen durch zahlreiche Neugründungen von Kolonien einen linearen Anstieg ihrer Abholzungsraten. Die geringste Rodungsfläche lässt sich den Japanern zuordnen, die, ausgehend von 1984, ihre landwirtschaftlichen Nutzflächen um etwa $50 \%$ auf $628 \mathrm{~km}^{2}$ erweitert haben (Tab.21, 22, Abb.17).

\subsubsection{Entwicklung der Landwirtschaftsflächen im Untersuchungsgebiet}

Tabelle 23 zeigt die wesentlichen Entwicklungen der Landbedeckung von 1984-2001. Bis 2001 beträgt die gesamte im Untersuchungsgebiet durch Rodungsaktivitäten betroffene Fläche ca. $16.000 \mathrm{~km}^{2}$. Diese Zahl schließt den Zeitraum vor 1984 mit ein. Wenig überraschend geht der größte Teil davon auf die landwirtschaftliche Expansion in den 1990er Jahren zurück. Allein $36 \%$ entfallen auf Anbauflächen, die längstenfalls seit 1992 genutzt werden und immerhin noch $10 \%$ auf Weiden, die 1992 mit Primärwald bestanden waren. Letztere konzentrieren sich überwiegend in den größeren Weidezonen um San Julian und San Ramon im Norden sowie im Süden in der Region Cabezas. Weitere $9 \%$ werden längstenfalls seit 1984 und $4 \%$ seit mehr als 17 Jahren kontinuierlich für den Anbau von Kulturen genutzt. Die meisten der am längsten in Kultur befindlichen Flächen befinden sich in der zentralen Zone, ein großer Teil davon in den japanischen und den älteren Kolonien der Mennoniten. Auf knapp 7 \% der Flächen wurde, vornehmlich in der nordwestlichen Kleinbauernzone, zum Folgezeitpunkt Sekundärwald klassifiziert. Das deutet darauf hin, dass innerhalb kurzer Zeitspannen Sekundärwälder aufwachsen, die nach GEROLD (1986) in den feuchteren Gebieten bereits nach 3 Jahren geschlossene Bestandshöhen von 5-6 Metern erreichen. Dass auch in den trockeneren Gebieten relativ schnell Sekundärwälder aufwachsen, zeigen mehrere große 2001 als Sekundärwald klassifizierte Bereiche in der südöstlichen agroindustriellen Anbauzone. Warum diese Flächen allerdings kurz nach deren Rodung in den 1990er Jahren 2001 wieder einen geschlossenen Sekundär-wald tragen, bleibt unklar. 4,5\% der in den 1990er Jahren gerodeten Flächen wurden 2001 als Bracheflächen klassifiziert und können als Indiz sich zum Ende der 1990er Jahre ändernder wirtschaftlicher Rahmenbedingungen angesehen werden (Kap.2.3.1). 4,2 \% der Flächen haben im Untersuchungszeitraum eine Nutzungsänderung von "Anbau" in "Weide" erfahren. Bei diesen handelt es sich um Flächen die bereits vor 1984 in Nutzung waren und offensichtlich infolge sinkender Bodenproduktivität nicht mehr für einen rentablen Anbau geeignet sind. In den Mennoniten-kolonien befinden sich Anbauflächen, die zwischenzeitlich als Weide genutzt wurden. Ob eine solche Wechsel- 
nutzung von den Mennoniten betrieben wird ist nicht bekannt. Dem Anschein nach handelt es sich eher um fehlklassifizierte Anbauflächen (Kap.4.2.1).

Die in ihrer Kombination nicht explizit aufgeführten $15 \%$ umfassen Entwicklungen, deren Anteil an der von Rodung betroffenen Gesamtfläche weniger als $1 \%$ betragen. Bei diesen handelt es sich bspw. um Konversionen in Siedlungsbereiche oder Brache- bzw. Sekundärwaldstadien während der Untersuchungsperiode. Demnach wurden 2001 weitere $562 \mathrm{~km}^{2}$ Anbau- und $685 \mathrm{~km}^{2}$ Weideflächen klassifiziert, während sich der Rest auf Bracheflächen, Sekundärwälder und Siedlungsbereiche aufteilt.

Tab. 23: Hauptsächliche Entwicklungen landwirtschaftlicher Nutzflächen im Zeitraum 1984-2001

\begin{tabular}{|c|c|c|c|c|c|c|}
\hline 1984 & & 1992 & & 2001 & $\mathbf{k m}^{2}$ & {$[\%]$} \\
\hline Primärwald & $\rightarrow$ & Primärwald & $\rightarrow$ & Anbau & 5.755 & 35,8 \\
\hline Primärwald & $\rightarrow$ & Primärwald & $\rightarrow$ & Weide & 1.594 & 9,9 \\
\hline Primärwald & $\rightarrow$ & Anbau & $\rightarrow$ & Anbau & 1.476 & 9,2 \\
\hline Primärwald & $\rightarrow$ & Primärwald & $\rightarrow$ & Brache & 716 & 4,5 \\
\hline Primärwald & $\rightarrow$ & Primärwald & $\rightarrow$ & Sekundärwald & 649 & 4,0 \\
\hline Anbau & $\rightarrow$ & Anbau & $\rightarrow$ & Anbau & 634 & 3,9 \\
\hline Weide & $\rightarrow$ & Weide & $\rightarrow$ & Weide & 512 & 3,2 \\
\hline Primärwald & $\rightarrow$ & Anbau & $\rightarrow$ & Weide & 383 & 2,4 \\
\hline Anbau & $\rightarrow$ & Weide & $\rightarrow$ & Weide & 349 & 2,2 \\
\hline Anbau & $\rightarrow$ & Anbau & $\rightarrow$ & Weide & 321 & 2,0 \\
\hline Primärwald & $\rightarrow$ & Weide & $\rightarrow$ & Weide & 321 & 2,0 \\
\hline Primärwald & $\rightarrow$ & Sekundärwald - & $\rightarrow$ & Sekundärwald & 249 & 1,6 \\
\hline Sekundärwald & $\rightarrow$ & Sekundärwald - & $\rightarrow$ & Sekundärwald & 195 & 1,2 \\
\hline Primärwald & $\rightarrow$ & Weide & $\rightarrow$ & Anbau & 181 & 1,1 \\
\hline Primärwald & $\rightarrow$ & Sekundärwald - & $\rightarrow$ & Anbau & 178 & 1,1 \\
\hline Anbau & $\rightarrow$ & Weide & $\rightarrow$ & Anbau & 165 & 1,0 \\
\hline andere & & & & & 2.381 & 14,8 \\
\hline$\Sigma$ & & & & & 16.062 & 100 \\
\hline
\end{tabular}

\subsubsection{Generalisierung der Landwirtschaftsflächen und Ableitung von Bewirtschaftungs- perioden}

Die Gebietsgröße bedingt, die Einzelfläche betreffend, eine Vielzahl an Entwicklungsgeschichten. Da mit Blick auf die Bodendegradationsanalyse nur die bewirtschafteten Flächen respektive deren Nutzungsentwicklung von Interesse sind, wurden Konversionen in Siedlungsbereiche und Sekundärwälder von der weiteren Untersuchung ausgenommen. Bracheflächen wurden generell in die Anbauklasse integriert, da über die Klassifikationsergebnisse mit wenigen Ausnahmen (53 $\left.\mathrm{km}^{2}\right)$ keine längeren Bracheperioden festgestellt wurden. Diese sollten nach GAUGUIN et al. (2002) mindestens 10-20 Jahre betragen um eine ausreichende Humusanreicherung im Boden zu gewährleisten. Für die verbliebenen Nutzungen ("Weide", "Anbau") ergaben sich 11 Klassen verschiedener Entwicklungen (Tab.24), die bestimmten Bewirtschaftungsperioden zugeordnet wurden. Die Einteilung dieser orientierte sich an den in Kap.5 ausgewerteten Literaturstudien und umfasst die Zeiträume weniger als 10 Jahre $(<10 \mathrm{a})$, 
zwischen 10 und 20 Jahren (10-20a) und länger als 20 Jahre (>20a) Nutzung. Die Landnutzungsklassifikationen der drei Aufnahmezeitpunkte ließen sich diesen Perioden gut zuordnen.

Tab. 24: Einteilung der Bewirtschaftungsperioden

\begin{tabular}{cccccccc}
\hline Klasse & $\mathbf{1 9 8 4}$ & & $\mathbf{1 9 9 2}$ & & $\mathbf{2 0 0 1}$ & Periode [a] & Anteil [\%] \\
\hline 1 & $*$ & & $*$ & & Weide & - & 12,8 \\
2 & $*$ & & $*$ & & Anbau & $<10 \mathrm{a}$ & 48,0 \\
3 & $*$ & & Weide & $\rightarrow$ & Weide & - & 3,0 \\
4 & $*$ & & Weide & $\rightarrow$ & Anbau & $<10 \mathrm{a}$ & 2,3 \\
5 & $*$ & & Anbau & $\rightarrow$ & Weide & $10-20 \mathrm{a}$ & 3,2 \\
6 & $*$ & & Anbau & $\rightarrow$ & Anbau & $10-20 \mathrm{a}$ & 12,0 \\
7 & Anbau & $\rightarrow$ & Anbau & $\rightarrow$ & Anbau & $>20 \mathrm{a}$ & 7,3 \\
8 & Anbau & $\rightarrow$ & Weide & $\rightarrow$ & Weide & $>20 \mathrm{a}$ & 3,0 \\
9 & Anbau & $\rightarrow$ & Anbau & $\rightarrow$ & Weide & $>20 \mathrm{a}$ & 2,5 \\
10 & Weide & $\rightarrow$ & Weide & $\rightarrow$ & Weide & - & 5,1 \\
11 & Weide & $\rightarrow$ & Anbau & $\rightarrow$ & Anbau & $10-20 \mathrm{a}$ & 0,9 \\
\hline $\boldsymbol{5}$ & & & & & & & $\mathbf{1 0 0}$ \\
\hline keine Nutzung & & & & & &
\end{tabular}

Die 2001 als "Weide" klassifizierten Bereiche wurden keiner Bewirtschaftungsperiode zugewiesen, da potenzielle Veränderungen bodenchemischer und -physikalischer Parameter unter Weidenutzug in der Bodendegradationsanalyse nicht näher quantifiziert werden. Eine Ausnahme bilden die bis 1984 bzw. 1992 ackerbaulich genutzten und 2001 als "Weide" ausgewiesenen Bereiche. Bei diesen, mehrheitlich in der zentralen Zone gelegenen Flächen, handelt es sich hypothetisch um degradierte Standorte mit einer Nutzungsgeschichte von mehr als 20 Jahren. Unsicherheiten bestehen bei den 1992 als "Anbau" und 2001 als "Weide" klassifizierten Bereichen (Klasse 5). Bei diesen kann bei einer Bewirtschaftungsdauer von maximal 17 Jahren nicht zwingend von zu Weideflächen degradierten Standorten ausgegangen werden. Vielmehr handelt es sich, bedingt durch den Aufnahmezeitpunkt, um z.T. unsicher klassifizierte Bereiche (Kap.4.2.1). Durch die vorangehende ackerbauliche Nutzung wurde diese in die 10- bis 20-jährige Bewirtschaftungsperiode eingeordnet. Ging den 2001 als "Anbau" klassifizierten Flächen eine Weidenutzung voraus, wurde diese nicht in die Bewirtschaftungsperiode eingerechnet. Dieser Einschätzung liegt die Tatsache zugrunde, dass mit Weidenutzung nicht per se negative Veränderungen bodenchemischer Eigenschaften einhergehen (MCGRATH et al. 2001, MÜLLER et al. 2004, MURTY et al. 2002).

Das aus den Ergebnissen der Landnutzungsklassifikationen abgeleitete Grid der verschiedenen Bewirtschaftungszeiträume dient nachfolgend als Grundlage bei der regionalquantitativen Analyse der Bodendegradation (Kap.5). 


\subsubsection{Zusammenfassung}

Die Ergebnisse zeigen für den Zeitraum von 1984 bis 2001 tiefgreifende Veränderungen der ursprünglichen Landschaft im ostbolivianischen Tiefland. Insgesamt wurden mehr als $12.000 \mathrm{~km}^{2}$ Primärwald in eine landwirtschaftliche Nutzung überführt, wobei der Hauptanteil der Konversion in Anbauflächen zuzuordnen ist. Demgegenüber ist der Anteil der Rodungen für Weidezwecke deutlich geringer. Das ist nicht überraschend, da sich die Weidewirtschaft hauptsächlich auf die natürlichen Graslandschaften der Chiquitania und des Chaco konzentriert. Im Untersuchungsgebiet zeigt sich eher die Tendenz, Anbauflächen bei nachlassender Bodenfruchtbarkeit in eine extensive Weidenutzung $\mathrm{zu}$ überführen. In zeitlicher Hinsicht gehen die höchsten Entwaldungsraten auf die 1990er Jahre zurück, in denen $72 \%$ der Rodungen festgestellt wurden. Ausgehend von der ersten landwirtschaftlichen Erschließungszone, der zentralen Zone, expandieren die landwirtschaftlichen Nutzflächen in der Hauptsache östlich des Río Grande, in der Expansionszone. Dort zeigen sich in den 1990er Jahren die mit Abstand höchsten Entwaldungsraten. Die zentrale Zone ist bis 1992, mit Ausnahme einiger verbliebener Waldinseln, nahezu vollständig entwaldet. Richtung Chaco konzentrieren sich die Agrarflächen, aufgrund humiderer Bedingungen, überwiegend in der Nähe zum Andenrand. In diesem Zusammenhang scheint sich mit zunehmender Entfernung vom Andenrand etwa der 18. Breitengrad als agronomische Trockengrenze zu manifestieren. Vereinzelt wird von Seiten der industriellen Landwirtschaft versucht, der geringeren Wasserverfügbarkeit im Chaco mit der Anlage großer Bewässerungssysteme zu begegnen.

In Bezug auf die Landnutzungssysteme gehen die höchsten Rodungsraten auf den agroindustriellen Sektor zurück. Die Rodungsraten der Mennoniten und Kleinbauern bewegen sich im Vergleich untereinander in einer ähnlichen Größenordnung. Etwa ein Drittel aller Rodungen im Untersuchungszeitraum gehen auf diese beiden Gruppen zurück. Auffällig in den Gebieten der Kleinbauern ist der hohe Anteil an Sekundärwäldern. Die geringste Flächenexpansion zeigt sich in den japanischen Kolonien. In regionaler Hinsicht expandieren die agroindustriellen Betriebe mehrheitlich in die Region der Chiquitania im Osten des Departments. Bei den Kleinbauern zeigt sich eine Konzentration im feuchteren Norden und Nordwesten, u.a. bedingt durch die dortigen geplanten Kolonien, sowie in geringerem Umfang in der Andenrandzone. Mennonitenkolonien sind mehr oder weniger gleichmäßig im Untersuchungsgebiet verteilt, wenngleich sich auch bei dieser Gruppe eine Tendenz erkennen lässt, eher Richtung Osten zu expandieren. Die Japaner haben keine weiteren Kolonien gegründet, expandieren folglich nur innerhalb der Grenzen ihrer bestehenden Kolonien in der zentralen Zone.

In zeitlicher Hinsicht wird knapp die Hälfte aller landwirtschaftlichen Nutzflächen am Ende der Untersuchungsperiode nicht länger als 9 Jahre genutzt. Die ältesten Agrarflächen 
befinden sich in der zentralen Zone, von denen bereits ein Großteil infolge abnehmender Bodenfruchtbarkeit zu Weideflächen degradiert ist. 


\section{BODENDEGRADATION}

Das nachfolgende Kapitel dient der Darstellung und Interpretation der im Untersuchungsgebiet als Folge landwirtschaftlicher Nutzung auftretenden Bodendegradationsprozesse. Diese werden für das Verständnis der Prozesskausalität vorab zusammengefasst (Kap.5.1). Die verschiedenen Formen der Bodendegradation im Untersuchungsgebiet sind vornehmlich das Ergebnis des durch Kultivierung bedingten Verlustes organischer Bodensubstanz sowie zunehmender Verdichtungen im Boden (BARBER 1995). Beide Prozesse werden im folgenden analysiert und deren wahrscheinlicher Status regional dargestellt.

Zunächst wird versucht, mittels geostatistischer Verfahren ursprüngliche, d.h. nicht durch Nutzung überprägte, Bodeneigenschaften aus den vorhandenen Profildaten in die Fläche zu generieren, um eine kontinuierliche metrische Ausgangsdatenbasis zu erhalten (Kap.5.1.1). Die Größenordnungen potenzieller Änderungen von Bodeneigenschaften infolge Kultivierung werden aus Literaturangaben entnommen und mit Ergebnissen aus dem Untersuchungsgebiet verglichen. Aus der Analyse werden Schätzgrößen der aktuellen Degradationsausprägung abgeleitet und unter Einbezug der Ergebnisse der Satellitenbildauswertung regional abgebildet (Kap.5.1.2, 5.1.3). Betrachtet wird in diesem Zusammenhang nur die Konversion in Anbauflächen, da Rodungen für Weidezwecke im Untersuchungsgebiet eine untergeordnete Rolle spielen. Abschließend wird versucht, potenziell winderosionsgefährdete Bereiche über die Ableitung eins Erosionsfaktors (K-Faktor) zu identifizieren (Kap.5.1.4).

\subsection{Bodendegradation im ostbolivianischen Tiefland}

Die Entwicklung des ostbolivianischen Tieflandes hin zu einer bedeutenden Landwirtschaftsregion in Bolivien begann, als Folge staatlicher Planungspolitik, erst vor ungefähr 50 Jahren (Kap.2.3). Dominierten zunächst traditionelle kleinbäuerliche Bewirtschaftungsformen mit einfachen slash and burn Systemen, traten in der Folge vermehrt vollmechanisierte Systeme zur Cash-Crop Produktion hinzu. Angetrieben durch die ökonomische Entwicklung und den technologischen Fortschritt wurden schnellwachsende Monokulturen oder stark vereinfachte Fruchtfolgen kultiviert, in deren Konsequenz zum Teil irreversible Degradationsschäden an den Böden aufgetreten sind. Diese äußern sich in Verdichtungen, dem Verlust organischer Substanz und daraus resultierend in einer Verringerung des Nährstoffpools. Die Böden werden generell anfälliger gegenüber Vernässung und Austrocknung bei gleichzeitig abnehmenden Erträgen.

Die schnelle Mineralisation organischer Substanz und der Anstieg der Lagerungsdichte infolge intensiver mechanischer Bodenbearbeitung gelten in dieser Region als die für die Degradation der Böden wesentlichen Prozesse. Zahlreiche ehemals produktive Anbauflächen, die nur noch als extensive Weideflächen genutzt werden können, zeugen von dieser Entwicklung. Allein für die zentrale Zone schätzt BARBER (1995) die Anzahl mittel bis 
schwer verdichteter Böden als Folge des mechanisierten Anbaus auf mehr als 50 \%. Dieselbe Entwicklung prognostiziert GEROLD (2002b) bei Fortdauer der gängigen Bearbeitungsmethoden der jüngeren landwirtschaftlichen Erschließungszone, der Expansionszone.

Die Prozesse der Bodendegradation sind nicht ausschließlich auf die Gebiete der industriellen Landwirtschaft beschränkt, sondern finden sich auch in den Gebieten der Kleinbauern, in erster Linie in den ehemals staatlichen Kolonien, wo sich in den letzten Jahren eine Tendenz zunehmender Mechanisierung bei gleichzeitiger Ablösung der traditionellen Landnutzungssysteme zeigt (MARTíNEZ 2004, URIOSTE \& PACHECO 2001).

\section{Bodendegradationsprozesse}

Mechanische Belastungen durch die industrielle Landwirtschaft haben auf den Agrarflächen der Alluvialebene z.T. Verdichtungen von 20-50 \% über den initialen Lagerungsdichten hervorgerufen (BARBER et al. 1989, BARBOSA et al. 1989). Diese resultieren aus einem zu häufigen Befahren mit schwerem Gerät (Schlepper, Pflug) bzw. der Bearbeitung des Bodens in einem nicht optimalen Zustand. Letzteres z.B. dann, wenn die Einsaat der Winterkultur während der Regenzeit, sprich bei feuchten Bodenverhältnissen erfolgt. Die Zerstörung des Bodengefüges durch den Aufbruch von Aggregaten macht die Böden anfällig gegenüber Wind- und Wassererosion. Direkte Folgen von Verdichtungen sind neben Beeinträchtigungen der biologischen Aktivität, Veränderungen der Porenverteilung und des Porenvolumens; dadurch sinkt die Infiltrationskapazität, während der Oberflächenabfluss steigt. Die Einschränkung des Porenraumes bedeutet gleichzeitig eine geringere Wasserverfügbarkeit für die Pflanze und Beeinträchtigungen im Wurzelwachstum, was bei längeren Trockenperioden zu erheblichen Ertragausfällen führen kann. Gleiches gilt, wenn nach der Aussaat hohe Niederschlagsmengen Staunässe im verdichteten Boden herbeiführen und das Saatgut regelrecht verfault. Bodenverdichtungen sind nicht ausschließlich auf Bearbeitungsprozesse zurückzuführen, sondern treten oft bereits als Folge unsachgemäß angewandter Rodungstechniken auf (BARBER \& ROMERO 1994).

Hohe Bodentemperaturen infolge der durch den Ackerbau geringeren Vegetationsbedeckung haben zusammen mit konventionellen Bodenbearbeitungsprozessen zu einer beschleunigten Mineralisation organischer Bodensubstanz und damit zum Abbau von Nährstoffspeichern und Gefügestabilisatoren im Boden geführt. Ein bestimmter Anteil geht dem Boden bereits während der Rodung durch einen lokal unterschiedlichen Abtrag des humusreichen A-Horizontes verloren (BARBER \& ROMERO 1994). Bodenwendende Bearbeitungsmaßnahmen ermöglichten zudem eine schnellere Mineralisation organischer Substanz auch tieferer Bodenschichten. Zusätzlich forciert wird der Humusabbau unter Kulturen wie Soja und Weizen, da bei diesen die mikrobielle Zersetzung deutlich die Menge der jährlich über Ernterückstände zugeführten organischen Substanz übersteigt (BARBER 1995). 
Der Anbau stark vereinfachter Fruchtfolgen in der industriellen Landwirtschaft ebenso wie der Export von Biomasse (Ernteentzug, Brand) aus dem System Pflanze-Boden, hat deutliche Nährstoffverluste nach sich gezogen (BARBER et al. 1996). Der Effekt verstärkt sich durch einen mehr oder weniger gänzlichen Verzicht von Düngergaben bei fast allen Landnutzungssystemen, durch zu kurze bzw. im System nicht vorgesehene Brachezeiten und durch die gängige Praxis Ernterückstände nicht auf den Feldern zu belassen sondern vom Vieh abweiden zu lassen bzw. zu verbrennen (BARBER \& NAVARRO 1994).

Das zeitweilige Fehlen einer geschlossenen Pflanzendecke fördert nach Niederschlägen die Ausbildung von Krusten an der Bodenoberfläche. Die Erosionskraft der Regentropfen lässt Aggregate zerfallen und das freigesetzte Feinmaterial blockiert die Bodenporen. Bei anschließender Austrocknung wird die Bodenoberfläche gewissermaßen versiegelt. Als Folge verstärkt sich der Oberflächenabfluss und somit die Erosion, während die Bodenfeuchte abnimmt. Die Bildung von Krusten ist auch das Ergebnis abnehmender Humusgehalte, wodurch die Aggregate einen schwächeren inneren Zusammenhalt haben und leicht zerfallen. Betroffen sind besonders Böden mit einem hohen Schluff- und Feinsandanteil sowie alle vegetationsfreien Bodenoberflächen ohne größere Aggregate (BARBER 1995).

Winderosion ist ein ernstes Problem in der Region. Eine hohe Gefährdung weisen vor allem die langjährig genutzten Böden der ersten landwirtschaftlichen Erschließungszone auf. Mit Beginn der agrarischen Nutzung vor etwa 50 Jahren wurde Winderosion nicht als Problem angesehen, da genug Waldfläche zur Abpufferung der in diesem Raum vorherrschenden starken Winde vorhanden war. Bis heute hat sich die Waldfläche so stark dezimiert, dass kein natürlicher Schutz mehr vorhanden ist. Hohe Windgeschwindigkeiten bewirken auf den v.a. in der Trockenzeit oft vegetationslosen Feldern erhebliche Abtragsraten. In jüngerer Zeit werden infolge gesetzlicher Vorgaben im Nachhinein Windschutzstreifen gepflanzt, deren Schutz allerdings aufgrund mangelnder Kenntnisse der Produzenten über Zusammensetzung und Pflanzabstand der infrage kommenden Bäume oft zweifelhaft ist. Bedauerlicherweise wird von Seiten des Gesetzgebers dazu keinerlei Beratung angeboten (WACHHOLtz 2000). Die agroindustriellen Betriebe versuchen diesem Problem in der jüngeren Erschließungszone mit der Anlage mächtiger Windschutzstreifen der natürlichen Waldvegetation vorzubeugen. Winderosion tritt vorzugsweise bei Böden mit einem hohen Sandanteil auf, da bei diesen die Summe stabiler Aggregate am geringsten ist; diese steigt mit zunehmenden Ton- und Schluffgehalten. Die Winderosion tritt nur bei trockenen Bodenverhältnissen und dem Fehlen einer Vegetationsdecke auf. Demnach ist besonders die Zeit nach der Aussaat kritisch. Unmittelbare Folgen sind der Verlust von Bodenmaterial und der organischen Substanz, woraus eine Abnahme der Bodenfruchtbarkeit resultiert. Als Problem erweist sich zudem die Ablagerung von Bodenpartikeln auf jungen Kulturen, die diese im Extremfall nicht zur Entwicklung kommen lassen (BARBER 1995). 


\subsubsection{Regionalisierung von Bodeneigenschaften}

Für den Erhalt einer kontinuierlichen metrischen Datenbasis wurde nachfolgend geprüft, ob punktuelle Bodeneigenschaften über die Ableitung von Schätzfunktionen aus Regressionsmodellen auf die Fläche übertragbar sind. Als unabhängige Variablen der Regression dienten die in den Kapiteln 3.3 und 3.4 beschriebenen Relief- und Klimaparameter, die jeweils als flächendeckende Rasterdatensätze vorlagen. Methodisch wurden die Profildaten mit den Flächendaten geostatistisch in Bezug gesetzt und über die Regressionsgleichung (Schätzfunktion) die Werte der zu regionalisierenden Variable (Zielvariable) bestimmt. Zielvariablen waren die unter natürlicher Vegetation im Oberboden gemessenen Sand-, Schluff- und Tongehalte, die organischen Kohlenstoff- (SOC) und Gesamtstickstoffgehalte $\left(\mathrm{N}_{\mathrm{t}}\right)$ sowie die Lagerungsdichten $\left(\mathrm{d}_{\mathrm{b}}\right)$. Auf deren Grundlage sollen mittlere, durch Kultivierung induzierte Veränderungen flächenhaft abgebildet werden. Die Anwendung dieses Verfahrens setzt die Annahme voraus, dass Relief- und Klimaparameter einen wesentlichen Einfluss auf die Bodenbildung haben und demnach die räumliche Anordnung von Böden beeinflussen.

Tab. 25: Korrelationskoeffizienten [r] von Klima- und Reliefparametern mit Oberbodenvariablen unter natürlicher Vegetation

\begin{tabular}{lcccccc}
\hline Variable & Sand [\%] & Schluff [\%] & Ton [\%] & SOC [\%] & $\mathbf{N}_{\mathbf{t}}[\%]$ & $\mathbf{d}_{\mathbf{b}}\left[\mathbf{g ~ c m}^{-3}\right]$ \\
& $n=191$ & $n=191$ & $n=191$ & $n=170$ & $n=155$ & $n=37$ \\
\hline Höhe [m] & $0,50^{* * *}$ & $-0,38^{* * *}$ & $-0,39 * * *$ & $-0,22 * *$ & n.s. & $-0,35^{*}$ \\
Hangneigung [\%] & $0,57 * * *$ & $-0,52 * * *$ & $-0,33^{* * *}$ & n.s. & n.s. & n.s. \\
AD [m] & $0,32 * * *$ & $-0,35 * * *$ & n.s. & n.s. & n.s. & n.s. \\
AS [m] & $-0,60^{* * *}$ & $0,52^{* * *}$ & $0,39 * * *$ & $0,17^{*}$ & n.s. & n.s. \\
NA [m] & $0,57^{* * *}$ & $-0,53^{* * *}$ & $-0,32^{* * *}$ & n.s. & n.s. & $-0,33^{*}$ \\
WI & $-0,69^{* * *}$ & $0,59 * * *$ & $0,46^{* * *}$ & $0,18^{*}$ & n.s. & $0,33^{*}$ \\
Niederschlag $(\mathbf{m m})$ & n.s. & $-0,22^{* *}$ & $0,13^{*}$ & $0,23^{* *}$ & n.s. & n.s. \\
Temperatur $\left[{ }^{\circ} \mathbf{C}\right]$ & $-0,55^{* * *}$ & $0,45^{* * *}$ & $0,40^{* * *}$ & $0,19^{*}$ & n.s. & $0,37 *$ \\
Strahlung $\left[\mathbf{J} / \mathbf{c m}^{2}\right]$ & $0,40^{* * *}$ & $-0,46^{* * *}$ & n.s. & $0,13^{*}$ & n.s. & n.s. \\
\hline
\end{tabular}

$\alpha<0,05 *, \alpha<0,01 * *, \alpha<0,001 * * *$ (n. PEARSON, einseitig)

Über lineare Einfachkorrelationen wurden die Variablen zunächst auf statistische Zusammenhänge überprüft (Tab.25). Deutlich wird eine signifikante Beziehung zwischen Reliefparametern und Bodenarten. Demnach scheint sich mit zunehmender Höhenlage eine Dominanz grobkörniger Substrate abzuzeichnen, während in der Alluvialebene feinere Bodenarten dominieren. Gleiches gilt für Hangbereiche, bei denen mit zunehmender Hangneigung gröbere Korngrößen anzutreffen sind, da feinere Partikel unter dem Einfluss der Schwerkraft verstärkt hangparallel verlagert werden. Diese wiederum akkumulieren sich in den lokalen Erosionsbasen, den Tiefenlinien [AS], demgegenüber lokale Scheitelbereiche [AD] eine Tendenz zu gröberen Sortierungen aufweisen. Ein offensichtlicher Zusammenhang besteht zwischen Bodenart und reliefbezogener Feuchteverteilung [WI $]$ im Boden. Höhere Bodenfeuchten gehen erkennbar mit feineren Korngrößen einher. Weniger eindeutig stellen 
sich die Zusammenhänge zwischen Bodenarten und klimatischen Parametern dar. Offenbar besteht eine schwache Tendenz höherer Tongehalte mit zunehmender Humidität. Die Zusammenhänge von Temperatur und Strahlung mit der Bodenart werden im wesentlichen auf die jeweiligen Höhengradienten zurückzuführen sein, da sich innerhalb der Alluvialebene weder Temperatur- noch Strahlungswerte im Jahresmittel nennenswert unterscheiden. Ausschlaggebend sind offensichtlich die sandigen Böden des brasilianischen Schildes (Abb.20), da in dieser Region gegenüber der Alluvialebene im Jahresmittel höhere Strahlungswerte und leicht geringere Temperaturen zu verzeichnen sind (Abb.10). Der scheinbare Zusammenhang abnehmender SOC-Gehalte mit zunehmender Höhe ist wiederum auf die sandigen Böden des brasilianischen Schildes zurückzuführen. Dies begründet sich durch die im Vergleich mit feinkörnigen Böden meist geringeren Humusgehalte sandiger Substrate (Kap.5.1.2). Der Effekt höherer Gehalte an organischer Substanz in feinkörnigen Böden kommt zudem in den Tiefenlinien $[\mathrm{AS}]$ bzw. beim Bodenfeuchteindex $\left[\mathrm{WI}_{\mathrm{S}}\right]$ zum tragen. Die positive Korrelation der SOC-Gehalte zum Niederschlag ergibt sich aus den höheren Humusgehalten der Böden in der nördlichen Alluvialebene (Tab.2). Dort hemmen im Vergleich mit dem südlichen Untersuchungsgebiet höhere Niederschläge und öfter auftretende Staunässe den Abbau organischer Bodensubstanz. Insgesamt scheinen die Zusammenhänge beim Kohlenstoff zu schwach, um aus den vorhandenen Daten robuste Schätzfunktionen abzuleiten. Gleiches gilt für die $\mathrm{N}_{\mathrm{t}^{-}}$ Gehalte und die Lagerungsdichten. Die $\mathrm{N}_{\mathrm{t}}$-Gehalte zeigen keine signifikanten Abhängigkeiten zu den erklärenden Variablen, obwohl ähnliche Abhängigkeiten wie beim Kohlenstoff zu erwarten wären, da Stickstoff im Oberboden zu über $90 \%$ in organischer Bindung vorliegt (SCHEFFER \& SchACHTSCHABEL 1998). Bei den Lagerungsdichten lässt allein der geringe Stichprobenumfang $(n=37)$ kaum sinnvolle Schätzungen erwarten.

Über eine schrittweise multiple Regressionsanalyse wurden Ziel- und Schätzvariablen in Beziehung gesetzt und, im Falle eines statistischen Zusammenhangs, Bodeneigenschaften über die abgeleitete Schätzfunktion in den Raum extrapoliert. Die besten Anpassungen wurden auf einem Signifikanzniveau von $\alpha=0,10$ erzielt. Die schwache Signifikanz musste aufgrund der geringen Datendichte im Untersuchungsgebiet akzeptiert werden. Wie bereits nach den Ergebnissen der Korrelationsanalyse zu erwarten war, ergaben sich zuverlässige Schätzfunktionen lediglich für die Sand- und Schluffgehalte (Abb.19). Diese erklären $50 \%$ (Sand) bzw. 37 \% (Schluff) der gefundenen Varianzen mit folgendenden Gleichungen:

$$
\begin{aligned}
& \text { Sand }[\%]=124,1+137,1 \beta-0,028 A S-3,49 W I_{S}\left(n=191, \mathrm{r}^{2}=0,495\right) \\
& \text { Schluff }[\%]=-7,4-128,6 \beta+0,02 A S+1,75 W I_{S}\left(n=191, \mathrm{r}^{2}=0,373\right) \\
\beta \quad= & \text { Hangneigung [\%] } \\
A S \quad= & \text { Reliefindex 'Höhe unter Scheitellinie' }
\end{aligned}
$$$$
W I_{S} \quad=\text { SAGA-Bodenfeuchte-Index }
$$ 
Bei allen anderen Parametern konnte die Regressionsfunktion nicht mit ausreichender Güte an die Daten angepasst werden $\left(r^{2}=0,08-0,22\right)$. Die Tongehalte wurden im GIS aus der Differenz der Summe der Sand- und Schluffgehalte zu 100 abgeleitet. Als Ergebnis der Regionalisierung standen flächendeckende Datensätze der Sand-, Schluff und Tongehalte zur Verfügung (Abb.20).
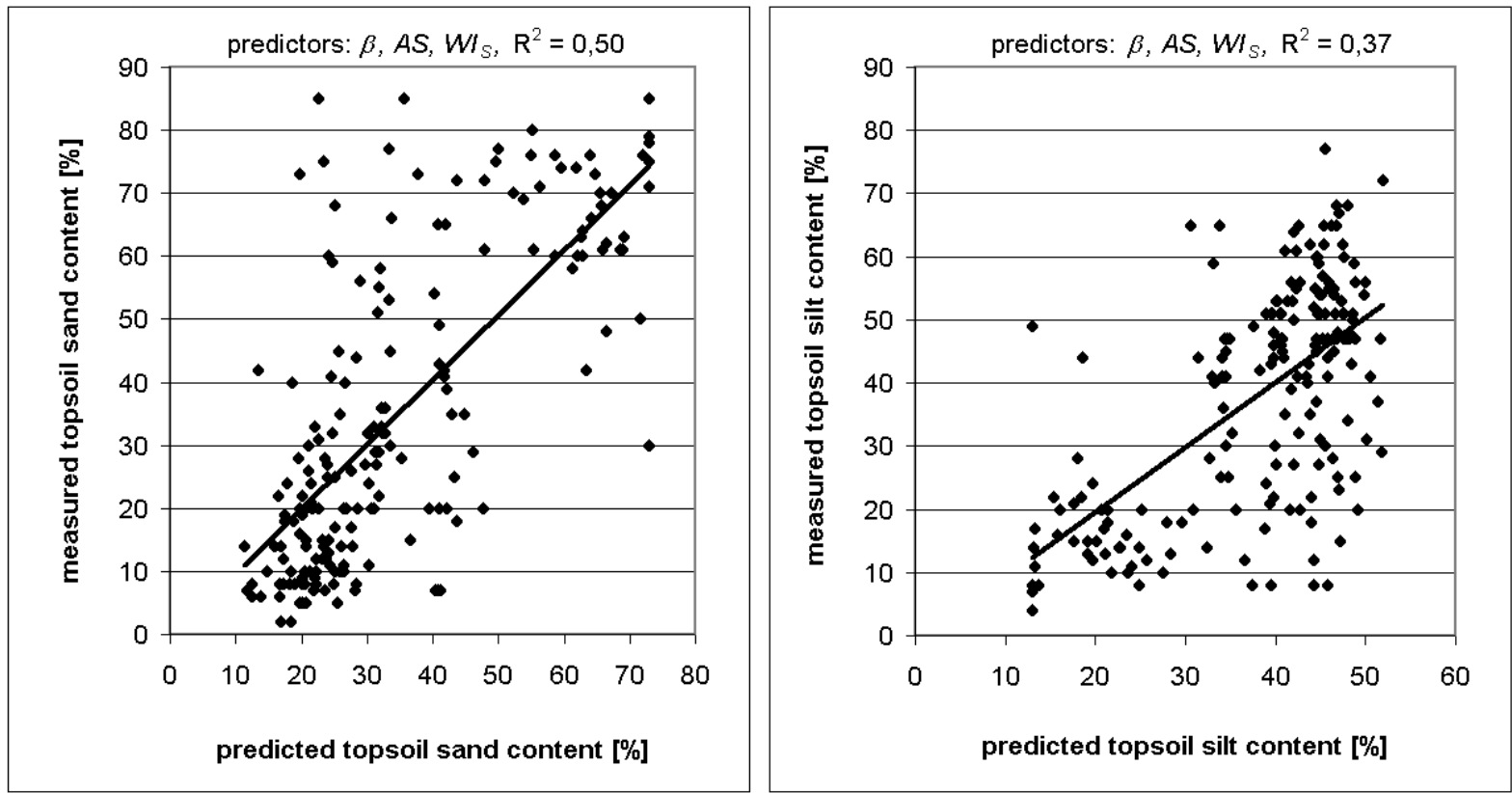

Abb. 19: Streudiagramm der gemessenen und regionalisierten Sand- und Schluffgehalte im Oberboden

Insgesamt sind die Ergebnisse der Bodenartenregionalisierung zufriedenstellend. Generell bestehen bei weniger hochauflösenden DGM Schwierigkeiten, Bodeneigenschaften in ebenem Gelände unter Nutzung topographischer Einflussgrößen zu schätzen (IsHIDA et al. 2003). Die z.T. kleinräumige Variabilität der Bodeneigenschaften in der Alluvialebene (Kap.2.2.2) kann über das in dieser Studie verwendete DGM (Rasterweite $185 \mathrm{~m}$ ) nicht erfasst werden. Stärkere Generalisierungen sind demnach unvermeidlich. Dennoch liefern die von BÖHNER \& KÖTHE (2003) entwickelten Reliefindizes mit erklärten Varianzen von 40-50 \% bei der Schätzung der Schluff- und Sandgehalte im Oberboden auch bei großen Gebietsgrößen mit vorherrschend flacher Topographie plausible Ergebnisse. Dies umso mehr vor dem Hintergrund der, im Verhältnis zur Gebietsgröße, geringen Anzahl von Bodendaten.

Bei den bodenchemischen Parametern $\left(\mathrm{SOC}, \mathrm{N}_{\mathrm{t}}\right)$ spielt neben der geringen Datenverfügbarkeit dem Anschein nach die Auflösung des DGM eine wesentliche Rolle. Bereits innerhalb kurzer Distanzen können die Gehalte an organischer Bodensubstanz hohen Variabilitäten unterliegen (Post et al. 2001), weshalb mit zunehmenden Rasterweiten potenzielle Zusammenhänge zwischen Relief und organischer Substanz nicht mehr erkannt werden. Im Untersuchungsgebiet wird diese Tatsache durch die ebene Topographie zusätzlich verstärkt. 

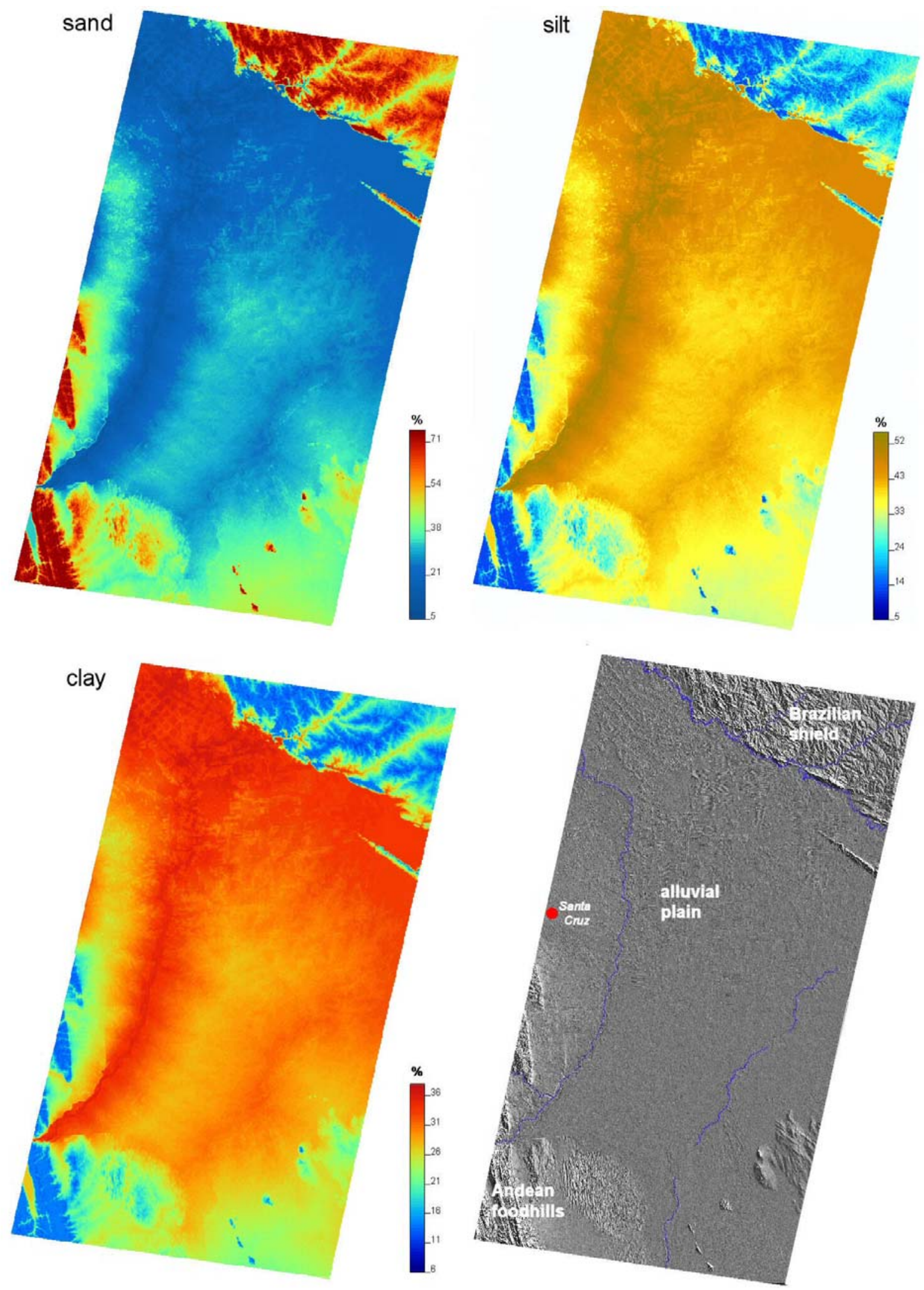

Abb. 20: regionalisierte Bodenarten und DGM des Untersuchungsgebiets 
Erfolgreiche Schätzungen des SOC- und $\mathrm{N}_{\mathrm{t}}$-Gehaltes erzielten BÖHNER \& SELIGE (2004) und VentARIS \& SLATER (2005) bei einer DGM-Rasterweite von $5 \mathrm{~m}$. Gleichzeitig merken Venteris \& SlATER (2005) jedoch an, dass die Topographie als Schätzvariable des SOCGehaltes insgesamt nur geringe Aussagekraft besitzt. Ein stärkerer Einfluss auf beide Bodenparameter wäre wegen der großen Heterogenität im Untersuchungsgebiet vom Niederschlag zu erwarten gewesen. Offensichtlich aber reicht dieser als erklärende Variable für die Kohlenstoff- und Stickstoffverteilung allein nicht aus. Signifikante Abhängigkeiten beider Parameter vom Niederschlag wurden von DALAL \& MAYER (1986a), KATYAL et al. (2001), Miller et al. (2004) und JobBÁGY \& JACKSON (2000) nachgewiesen. Das die ungleichen Oberbodenmächtigkeiten der untersuchten Profile die Variabilität der SOC- und $\mathrm{N}_{\mathrm{t}}$-Gehalte nennenswert beeinflussen wird ausgeschlossen, da zwischen den Oberbodenmächtigkeiten und dem Kohlenstoff- bzw. Stickstoffgehalt im Boden kein korrelierter Zusammenhang besteht.

Die regionalisierten Bodenarten bilden in der weiteren Analyse die Grundlage der Anwendung von Degradationsszenarien. Für die Parameter Kohlenstoff, Stickstoff und Lagerungsdichte war eine plausible räumliche Schätzung auf der gewählten Maßstabsebene nicht möglich. Für diese werden demnach allgemeine, bodenartenabhängige Mittelwerte aus den vorhandenen Daten abgeleitet und als Ausgangsniveau unter natürlicher Vegetation vorausgesetzt (Kap.5.1.2.2).

\subsubsection{Zusammenfassung}

Die Regionalisierung von Sand- und Schluffgehalten unter Nutzung von Reliefvariablen liefert zufriedenstellende Ergebnisse. Die erklärten Varianzen liegen mit 40-50\% in einem Bereich, der bei der untersuchten Gebietsgröße, der geringen Datendichte und der unterschiedlichen Datenqualität (Kap.3) kaum höher sein kann. Der Flächendatensatz für Ton wurde rechnerisch über die Sand- und Schluffgehalte ermittelt. Die Klimavariablen lieferten bezüglich der regionalen Verbreitung der Bodenarten keinen zusätzlichen Erklärungsgehalt. Die SOC- und $\mathrm{N}_{\mathrm{t}}$-Gehalte ebenso wie die Lagerungsdichten konnten wegen $\mathrm{zu}$ niedriger erklärter Varianzen mit den Schätzvariablen nicht räumlich extrapoliert werden. Für diese werden in der Folge allgemeine aus den verfügbaren Bodendaten abgeleitete Werte als Ausgangsniveau angenommen (Kap.5.1.2.2 und Kap.5.1.3.1). 


\subsection{2 Änderung von SOC und $\mathrm{N}_{\mathrm{t}}$ unter ackerbaulicher Nutzung in den Tropen und Sub- tropen}

Zahlreiche Studien belegen, dass mit der Konversion natürlicher Vegetation in Kulturflächen eine Abnahme der organischen Substanz (SOM) im Boden einhergeht. Die meisten Autoren fokussieren in diesem Zusammenhang auf den organischen Kohlenstoffgehalt (DAVIDSON \& ACKERMAN 1993, GUO \& GIFFORD 2002), dessen Anteil an der organischen Substanz (SOM) tropischer Böden ungefähr bei 58 \% liegt (LANDON 1984). Andere beziehen zusätzlich den Stickstoffgehalt in ihre Analyse mit ein (MURTY et al. 2002). In Böden tropischer Regionen liegen die Mineralisationsraten organischer Substanz aufgrund der wärmeren und feuchteren Bedingungen 2-5 mal so hoch, wie in den gemäßigten Breiten (BAYER et al. 2001, SIX et al. 2002). Folglich sind bereits in den ersten Jahren ackerbaulicher Nutzung die höchsten Verluste an organischer Bodensubstanz zu verzeichnen, während sich in den Folgejahren ein nur noch gradueller Rückgang anschließt.

LOBE et al. (2001) ermittelten in Oberböden von Alfisolen in Südafrika eine Reduktion des SOC-Gehaltes um $49 \%$ in den ersten 10 Anbaujahren und um $58 \%$ nach 45 Jahren. BondE et al. (1992) berichten von SOC-Verlusten nach 12 bzw. 50 Jahren unter kontinuierlichem Zuckerrohranbau auf Oxisolen in Südost-Brasilien von 56 \% bzw. 59 \%. In diesem Zusammenhang scheint weniger der Grad der Mechanisierung bei der Bearbeitung der Böden eine Rolle zu spielen, als vielmehr der Einsatz des Pfluges im allgemeinen. So kommen SAIKH et al. (1998) auf traditionell bewirtschafteten Flächen ohne Einsatz moderner Technologie auf Alfisolen in Ost-Indien auf SOC-Verluste in der Größenordnung von $41 \%$ (5 a), $65 \%$ (15 a) und $70 \%$ (30 a). Gleiches berichtet JAIYEOBA (2003) in einer Studie über die traditionelle Bewirtschaftungsweise von Kleinbauern in Nigeria. Seinen Ergebnissen zufolge reduziert sich der SOC-Gehalt nach 25 Jahren um $60 \%$, wovon über die Hälfte auf die ersten drei Anbaujahre zurückgeht. Die initialen Verluste treten in der Regel so schnell auf, dass diese auch auf Flächen mit kurzer Nutzungsdauer (z.B. slash and burn Systeme) im Mittel in einer ähnlichen Größenordnung liegen, wie unter anderen Bewirtschaftungsformen (DAVIDSON \& ACKERMAN 1993). Nach ca. 20 bis 30 Jahren kontinuierlichem Anbau stellt sich unter den veränderten Bedingungen ein neuer Gleichgewichtszustand bei Anlieferung und Abbau der organischen Substanz im Boden ein. Die jährliche SOC-Reduktion liegt zu diesem Zeitpunkt im Mittel bei $2 \%$ (DOMINY et al. 2002, LEMENIH et al. 2005, LOBE et al. 2001, MANN 1986).

Die zeitlichen Änderungen der Gesamtstickstoffgehalte zeigen unter kontinuierlichem Anbau denselben Verlauf wie die des organischen Kohlenstoffs. Auf hohe initiale Verluste folgt eine nur noch allmähliche Reduktion der $\mathrm{N}_{\mathrm{t}}$-Gehalte. Wie beim organischen Kohlenstoffgehalt stellt sich nach 20 bis 30-jähriger Ackernutzung auch beim Stickstoff ein neuer Gleichgewichtszustand im Boden mit Abbauraten unter $2 \%$ pro Jahr ein (LOBE et al. 2001). Die Gesamtverluste liegen zu diesem Zeitpunkt im Mittel bei $50 \%$, sofern keine zusätzlichen Düngergaben erfolgten (LEMENIH et al 2005, SAIKH et al. 1998). 
Tab. 26: SOC- und $\mathrm{N}_{\mathrm{t}}$-Verluste im Oberboden nach Konversion natürlicher Vegetation in eine ackerbauliche Nutzung im tropischen Tiefland von Ostbolivien

\begin{tabular}{|c|c|c|c|c|c|c|c|}
\hline \multirow{2}{*}{$\begin{array}{l}\text { Region (Bodentyp, -art) } \\
\text { Bodentiefe, Produzent }\end{array}$} & \multirow{2}{*}{$\begin{array}{c}\text { Nutzdauer } \\
\text { Jahre }\end{array}$} & \multirow{2}{*}{$\begin{array}{c}\text { SOC } \\
{\left[\mathrm{g} \mathrm{kg}^{-1}\right]}\end{array}$} & \multicolumn{2}{|c|}{ Verlust } & \multirow{2}{*}{$\begin{array}{c}\mathbf{N}_{\mathbf{t}} \\
{\left[\mathrm{g} \mathrm{kg}^{-1}\right]}\end{array}$} & \multicolumn{2}{|c|}{ Verlust } \\
\hline & & & $\begin{array}{c}\text { gesamt } \\
{[\%]}\end{array}$ & $\begin{array}{c}\text { pro Jahr } \\
{[\%]}\end{array}$ & & $\begin{array}{c}\text { gesamt } \\
{[\%]}\end{array}$ & $\begin{array}{c}\text { pro Jahr } \\
{[\%]}\end{array}$ \\
\hline${ }^{1}$ Las Brechas (Alfisol, SL) & + & 1,62 & $*$ & $*$ & 0,18 & * & $*$ \\
\hline \multirow[t]{4}{*}{ 0-30 cm, Mennoniten } & 5 & 1,04 & 36 & 7,2 & 0,13 & 28 & 5,6 \\
\hline & 15 & 0,84 & 48 & 3,2 & 0,13 & 31 & 2,0 \\
\hline & 25 & 0,61 & 63 & 2,5 & 0,10 & 47 & 1,9 \\
\hline & 29 & 0,64 & 61 & 2,1 & 0,09 & 50 & 1,7 \\
\hline${ }^{2}$ Saavedra (Alfisol, SL) & + & 1,61 & $*$ & $*$ & 0,18 & $*$ & * \\
\hline \multirow[t]{2}{*}{ 0-15 cm, Agroindustrie } & 3 & 0,93 & 42 & 14,0 & 0,10 & 44 & 14,7 \\
\hline & 6 & 0,83 & 48 & 8,0 & 0,10 & 44 & 7,3 \\
\hline${ }^{3}$ Las Brechas (Vertisol, C) & + & * & * & * & 0,20 & * & * \\
\hline 0-20 cm, Agroindustrie & 23 & $*$ & $*$ & $*$ & 0,14 & 30 & 1,3 \\
\hline${ }^{3}$ Las Brechas (Alfisol, SCL) & + & * & * & $*$ & 0,18 & * & $*$ \\
\hline $0-30 \mathrm{~cm}$, Agroindustrie & 23 & $*$ & $*$ & $*$ & 0,08 & 56 & 2,4 \\
\hline \multirow{2}{*}{$\begin{array}{l}{ }^{4} \text { Las Brechas (Inceptisol, SL) } \\
0-15 \mathrm{~cm} \text {, Mennoniten }\end{array}$} & + & 0,99 & * & $*$ & $*$ & * & * \\
\hline & 18 & 0,46 & 53 & 2,9 & $*$ & $*$ & $*$ \\
\hline \multirow{2}{*}{$\begin{array}{l}{ }^{4} \text { Las Brechas (Alfisol, SCL) } \\
0-15 \mathrm{~cm} \text {, Mennoniten }\end{array}$} & + & 1,45 & $*$ & $*$ & * & * & $*$ \\
\hline & 14 & 0,93 & 36 & 2,6 & $*$ & * & $*$ \\
\hline \multirow{2}{*}{$\begin{array}{l}{ }^{4} \text { Saavedra (Alfisol, SL) } \\
0-15 \mathrm{~cm} \text {, Agroindustrie }\end{array}$} & + & 1,80 & $*$ & $*$ & $*$ & * & $*$ \\
\hline & 5 & 0,81 & 55 & 11,0 & $*$ & $*$ & $*$ \\
\hline \multirow{2}{*}{$\begin{array}{l}{ }^{4} \text { Saavedra (Inceptisol, SL) } \\
0-15 \mathrm{~cm} \text {, Agroindustrie }\end{array}$} & + & 2,30 & $*$ & $*$ & $*$ & $*$ & $*$ \\
\hline & 29 & 0,64 & 72 & 2,5 & $*$ & $*$ & $*$ \\
\hline \multirow{3}{*}{$\begin{array}{l}{ }^{5} \text { Las Brechas (Alfisol, L) } \\
0-30 \mathrm{~cm} \text {, Kleinbauern }\end{array}$} & + & 2,55 & $*$ & $*$ & 0,30 & $*$ & $*$ \\
\hline & 5 & 1,16 & 55 & 11,0 & 0,13 & 57 & 11,4 \\
\hline & 7 & 1,22 & 52 & 7,4 & 0,14 & 53 & 7,6 \\
\hline \multirow{3}{*}{$\begin{array}{l}{ }^{6} \text { Lomerio (Inceptisol, SL) } \\
0-20 \mathrm{~cm}, \text { Kleinbauern }\end{array}$} & + & 3,00 & $*$ & $*$ & 0,28 & $*$ & $*$ \\
\hline & 7 & 2,00 & 33 & 4,8 & 0,17 & 39 & 5,6 \\
\hline & 24 & 1,30 & 57 & 2,4 & 0,09 & 68 & 2,8 \\
\hline \multirow{4}{*}{$\begin{array}{l}{ }^{7} \text { San Julian (k. A., SIC) } \\
0-30 \mathrm{~cm}, \text { Kleinbauern }\end{array}$} & + & 2,73 & $*$ & $*$ & 0,24 & $*$ & $*$ \\
\hline & 3 & 2,20 & 19 & 6,4 & 0,19 & 21 & 6,9 \\
\hline & 4 & 2,38 & 13 & 3,2 & 0,21 & 13 & 3,1 \\
\hline & 8 & 1,16 & 57 & 7,2 & 0,10 & 58 & 7,3 \\
\hline \multirow{6}{*}{$\begin{array}{l}{ }^{7} \text { Okinawa (k. A., C) } \\
\text { 0-30 cm, Kleinbauern } \\
{ }^{7} \text { Yapacani (k. A., CL, SIC) } \\
\text { 0-30 cm, Kleinbauern }\end{array}$} & + & 2,73 & $*$ & $*$ & 0,24 & $*$ & $*$ \\
\hline & 3 & 2,32 & 15 & 5,0 & 0,20 & 17 & 5,6 \\
\hline & + & 1,60 & * & * & 0,14 & * & $*$ \\
\hline & 3 & 0,99 & 38 & 12,8 & 0,09 & 36 & 11,9 \\
\hline & + & 2,67 & $*$ & $*$ & 0,20 & $*$ & $*$ \\
\hline & 15 & 1,51 & 43 & 2,9 & 0,09 & 55 & 3,7 \\
\hline \multirow{2}{*}{$\begin{array}{l}{ }^{7} \text { San Pedro (k. A., SICL) } \\
0-30 \mathrm{~cm} \text {, Kleinbauern }\end{array}$} & + & 1,86 & $*$ & $*$ & 0,16 & $*$ & $*$ \\
\hline & 5 & 1,39 & 25 & 5,0 & 0,12 & 25 & 5,0 \\
\hline \multirow{2}{*}{$\begin{array}{l}{ }^{7} \text { Río Grande (k. A., SIL) } \\
\text { 0-30 cm, Kleinbauern }\end{array}$} & + & 1,39 & $*$ & $*$ & 0,13 & $*$ & $*$ \\
\hline & 3 & 1,16 & 17 & 5,6 & 0,10 & 23 & 7,7 \\
\hline
\end{tabular}

MARKussen (2000); ${ }^{2}$ Barber et al. (1996); ${ }^{3}$ Guaman (1999); ${ }^{4}$ Barber (1995); ${ }^{5}$ KARsten (2000); ${ }^{6}$ BlaschKe (1998);

${ }^{7}$ CIAT (1980) +: Ausgangsgehalt unter natürlicher Vegetation $\quad$ k. A.: keine Angabe

Bodenarten: SL: sandy loam, SCL: sandy clay loam, L: loam, CL: clay loam, SIL: silty loam, SICL, silty clay loam, SIC: silty clay, C: clay (nach US Soil Tax)

Verschiedene Autoren konstatieren eine enge Korrelation der SOC- und $\mathrm{N}_{\mathrm{t}}$-Verluste in Abhängigkeit von der Nutzungsdauer, wobei die Gesamtabnahmebeträge des SOC nach langjähriger Ackernutzung im allgemeinen über denen des Gesamtstickstoffs liegen (LOBE et al 2001, MurTy et al. 2002, ZhAO et al. 2005). Die gleiche Tendenz ist auch bei den im Unter- 
suchungsgebiet durchgeführten Studien erkennbar. Annähernd $40 \%$ der Gesamtverluste gehen auf die ersten 10 Anbaujahre zurück, während sich die Gehalte nach 20-30 Jahren auf einem mittleren Niveau von 50 \% der Ausgangsgehalte einstellen (Tab.26).

\subsubsection{Ableitung relativer SOC und $\mathrm{N}_{\mathrm{t}}$-Verluste - Literaturreview}

Wegen der geringen Stichprobenzahl und der daraus folgenden geringen Repräsentativität der im Untersuchungsgebiet durchgeführten Studien zur Abnahme der organischen Bodensubstanz mit Dauer der ackerbaulichen Nutzung, werden die Werte nachfolgend mit den Ergebnissen anderer in tropischen und subtropischen Breiten durchgeführten Studien verglichen und in einer gemeinsamen Datenbank angeordnet. Die Tabelle mit den daraus abgeleiteten prozentualen Abnahmebeträgen für SOC und $\mathrm{N}_{\mathrm{t}}$ im Oberboden befindet sich im Anhang (Tab.A5, Anhang). Die Werte dienen als Größenordnung für die flächenhafte Abschätzung der durch den Ackerbau ausgelösten Abnahme organischer Substanz im Untersuchungsgebiet.

Tab. 27: Mittlere prozentuale Rückgänge der SOC- und $\mathrm{N}_{\mathrm{t}}$-Gehalte unter moderner und traditioneller Bewirtschaftung nach mehr als 10 Jahren (10-70 a) kontinuierlicher Nutzung (Oberboden)

\begin{tabular}{llcccccc}
\hline & & \multicolumn{2}{c}{ modern } & \multicolumn{2}{c}{ traditionell } & $\Delta[\%]$ & $\Delta[\%]$ \\
& & gesamt & pro Jahr & gesamt & pro Jahr & $\begin{array}{c}\Delta[\%] \\
\text { gesamt }\end{array}$ & $\begin{array}{c}\text { pro Jahr } \\
\text { SOC }\end{array}$ \\
& MW & 49,6 & 2,5 & 49,3 & 2,5 & 0,5 & $-0,1$ \\
& Stabw & 13,4 & 1,1 & 17,7 & 1,3 & & \\
& $\mathrm{n}$ & 45 & 45 & 25 & 25 & & \\
$\mathbf{N}_{\mathbf{t}}$ & MW & 43,8 & 2,1 & 48,9 & 2,5 & $-5,0$ & $-0,4$ \\
& Stabw & 10,1 & 0,8 & 18,1 & 1,3 & & \\
& $\mathrm{n}$ & 29 & 29 & 19 & 19 & & \\
\hline
\end{tabular}

Aus der Literatur wurden inklusive der in Bolivien durchgeführten Untersuchungen 40 Studien ausgewählt, von denen insgesamt 117 Stichproben in die Auswertung einbezogen wurden. Die meisten beziehen sich auf den organischen Kohlenstoff $(n=112)$ und weniger auf den Gesamtstickstoff $(n=69)$. Die Studien wurden entweder als paarweiser Vergleich, bei dem kultivierte Flächen mit benachbarten Flächen natürlicher Vegetation verglichen werden, oder als Chronosequenz (falsche Zeitreihe) durchgeführt. Letztere untersuchen Flächen unterschiedlich langer Nutzungsperioden und vergleichen diese mit einer in der Nähe befindlichen Fläche natürlicher Vegetation (MANN 1986). Die Daten decken einen Nutzungszeitraum von 2 bis 70 Jahren ab und repräsentieren ein Spektrum sandig bis toniger Böden. Die Probentiefen reichen bis maximal $30 \mathrm{~cm}$. Eine Unterteilung in traditionelle und moderne Landnutzungssysteme wurde nicht vorgenommen, da bei allen analysierten Studien konventionelle, d.h. nicht bodenschonende Bewirtschaftungstechniken zur Anwendung kommen (Pflugsysteme). Ein Vergleich beider Anbauverfahren nach kontinuierlicher Nutzung von 10 
und mehr Jahren belegt, dass in Bezug auf mittlere SOC- und $\mathrm{N}_{\mathrm{t}}$-Verluste keine nennenswerten Unterschiede zwischen der Kultivierung mit Ochsen oder Pferden bzw. Traktoren bestehen (Tab.27). Die geringeren $\mathrm{N}_{\mathrm{t}}$-Verluste der modernen Systeme resultieren aus Düngerapplikationen.

Nach DAVIDSON \& ACKERMAN (1993) ist die Angabe der Konzentration (SOC- und $\mathrm{N}_{\mathrm{t}^{-}}$ Gehalt) kein genauer Indikator bei der Berechnung von Änderungen gegenüber dem Ausgangsniveau, da bei der Berechnung die Lagerungsdichte $\left(\mathrm{d}_{\mathrm{b}}\right)$ unberücksichtigt bleibt und tatsächliche $\mathrm{C}$ - und $\mathrm{N}$-Verluste folglich oft überschätzt werden. Zuverlässigere Ergebnisse lassen sich über den Vergleich der jeweiligen Bodenvorräte erzielen, da diesen bei der Berechnung über den Einbezug der Bodenmasse ein einheitlicher Bezug zugrunde liegt. Sofern die Daten der analysierten Studien nicht bereits in Masse pro Fläche vorlagen, wurden die prozentualen Gehaltsangaben über die Lagerungsdichte und Horizontmächtigkeit nach folgender Formel errechnet:

$$
\operatorname{SOC}_{\mathrm{i}}=\mathrm{d}_{\mathrm{b}} * \operatorname{SOC}_{\mathrm{c}} * \mathrm{~h}
$$

$\mathrm{SOC}_{\mathrm{i}}$ : organischer C-Vorrat $\left[\mathrm{tha}^{-1}\right]$

$\mathrm{SOC}_{\mathrm{c}}$ : organischer C-Gehalt [\%]

$\mathrm{d}_{\mathrm{b}} \quad$ : Lagerungsdichte $\left[\mathrm{g} \mathrm{cm}^{-3}\right]$

$\mathrm{h} \quad$ : Horizontmächtigkeit $[\mathrm{cm}]$

Die $\mathrm{N}_{\mathrm{t}}$-Vorräte berechnen sich äquivalent zu denen des organischen Kohlenstoffs. Lagen keine Angaben zur Lagerungsdichte vor $(n=15)$, wurde die Werte nach ADAMS $(1973)^{25}$ näherungsweise wie folgt bestimmt:

$$
d_{b}=\frac{100}{\frac{S O M}{0,244}+\frac{100-S O M}{1,64}}
$$

SOM: organische Substanz [\%]

wobei der Faktor 0,224 die angenommene Dichte der organischen Substanz in $\left[\mathrm{g} \mathrm{cm}^{-3}\right]$ und der Faktor 1,64 die für die Berechnung verwendete Dichte des Mineralbodens in $\left[\mathrm{g} \mathrm{cm}^{-3}\right]$ darstellen. Der organische Kohlenstoffgehalt im Boden wurde mit dem Faktor 1,72 auf Prozent organische Substanz umgerechnet (LANDON 1984). Der Vergleich prozentualer Abnahmebeträge von Konzentrationen und Vorräten der zugrunde liegenden Studien bestätigt die Ergebnisse von DAVIDSON \& ACKERMAN (1993), dass allein auf Basis von C- und NGehalten ermittelte Verluste höher ausfallen, als wenn Vorratsangaben die Grundlage der

\footnotetext{
${ }^{25}$ zitiert in (GUO \& GIFFORD 2002), MANN (1986), POST \& KWON (2000)
} 
Berechnungen bilden. Im Mittel errechnen sich aus den Bodenvorräten etwa $5 \%$ geringere Verluste (Tab.29).

Tab. 28: Korrelationsmatrix von Gesamtverlusten und jährlicher Raten für SOC und $\mathbf{N}_{\mathrm{t}}$ aller analysierten Studien (Oberboden)

\begin{tabular}{|c|c|c|c|c|c|c|c|c|c|c|c|c|}
\hline & & & $\begin{array}{c}\text { Tiefe } \\
\mathrm{cm}\end{array}$ & $\begin{array}{c}\text { Nutzung } \\
\mathrm{a}\end{array}$ & $\begin{array}{l}\text { SOC } \\
\mathrm{g} \mathrm{kg}^{-1}\end{array}$ & $\mathrm{~g} \mathrm{~kg}^{-1} \mathrm{a}^{-1}$ & $\mathrm{tha}^{-1}$ & tha $^{-1} a^{-1}$ & $\begin{array}{c}\mathbf{N}_{\mathbf{t}} \\
\mathrm{g} \mathrm{kg}^{-1}\end{array}$ & $\mathrm{~g} \mathrm{~kg}^{-1} \mathrm{a}^{-1}$ & $\mathrm{tha}^{-1}$ & $t h a^{-1} a^{-1}$ \\
\hline \multirow[t]{7}{*}{ SOC } & $\mathrm{g} \mathrm{kg}^{-1}$ & $\begin{array}{l}\mathrm{r} \\
n\end{array}$ & $\begin{array}{l}\text { n.s. } \\
99\end{array}$ & $\begin{array}{c}0,46 * * * \\
101\end{array}$ & & & & & & & & \\
\hline & $\mathrm{g} \mathrm{kg}^{-1} \mathrm{a}^{-1}$ & $\mathrm{r}$ & $0,20^{*}$ & $-0,86^{* * *}$ & - & & & & & & & \\
\hline & & $n$ & 99 & 101 & - & & & & & & & \\
\hline & $\mathrm{tha}^{-1}$ & $\mathrm{r}$ & n.s. & $0,45^{* * *}$ & $0,95^{* * *}$ & - & & & & & & \\
\hline & & $n$ & 112 & 112 & 98 & - & & & & & & \\
\hline & $\mathrm{tha}^{-1} \mathrm{a}^{-1}$ & $\mathrm{r}$ & n.s. & $-0,84 * * *$ & - & $0,97^{* * *}$ & - & & & & & \\
\hline & & $n$ & 112 & 112 & - & 98 & - & & & & & \\
\hline \multirow[t]{8}{*}{$N_{t}$} & $\mathrm{~g} \mathrm{~kg}^{-1}$ & $\mathrm{r}$ & n.s. & $0,49 * * *$ & $0,76^{* * *}$ & - & - & - & & & & \\
\hline & & $n$ & 68 & 68 & 66 & - & - & - & & & & \\
\hline & $\mathrm{g} \mathrm{kg}^{-1} \mathrm{a}^{-1}$ & $\mathrm{r}$ & n.s. & $-0,89^{* * *}$ & - & $0,92 * * *$ & - & - & - & & & \\
\hline & & $n$ & 68 & 68 & - & 66 & - & - & - & & & \\
\hline & $\mathrm{tha}^{-1}$ & $\mathrm{r}$ & $-0,23^{*}$ & $0,43 * * *$ & - & - & $0,56^{* * *}$ & - & $0,74^{* * *}$ & - & & \\
\hline & & $n$ & 69 & 69 & - & - & 69 & - & 66 & - & & \\
\hline & $t h a^{-1} a^{-1}$ & $\mathrm{r}$ & n.s. & $-0,89 * * *$ & - & - & - & $0,88^{* * *}$ & - & $0,91 * * *$ & - & \\
\hline & & $n$ & 69 & 69 & - & - & - & 69 & - & 66 & - & \\
\hline \multirow[t]{2}{*}{ Ton } & $\%$ & $\mathrm{r}$ & - & - & $-0,28 * *$ & - & $-0,23 *$ & - & $-0,24^{*}$ & - & n.s. & - \\
\hline & & $n$ & - & - & 87 & - & 97 & - & 61 & - & 61 & - \\
\hline
\end{tabular}

$\alpha>0,05$ n.s., $\alpha<0,05 *, \alpha<0,01 * *, \alpha<0,001 * * *$ (n. SPEARMAN, einseitig)

\section{Einfluss der Bodentiefe}

Eine signifikante Abhängigkeit der Verluste von der beprobten Horizontmächtigkeit im Oberboden ist über die Daten nicht festzustellen $(\alpha>0,05)$. Die relativen Abnahmebeträge scheinen, unabhängig davon, ob die obersten zehn oder dreißig Zentimeter des Bodens beprobt wurden, mehr oder weniger gleich zu sein. Beim SOC-Gehalt sind sogar höhere Verluste mit zunehmender Horizontmächtigkeit zu verzeichnen. Einzig bei den $\mathrm{N}_{\mathrm{t}}$-Vorräten zeigt sich eine Verringerung der relativen Verluste mit zunehmender Mächtigkeit des beprobten Horizontes (Tab.28). Offenbar reduzieren sich die mittleren Verluste organischer Substanz erst unterhalb des Pflughorizontes. MURTY et al. (2002) ermittelten nach langjähriger Nutzung für die obersten 15 Bodenzentimeter im Mittel $26 \%$ höhere SOC-Verluste im Vergleich mit einer beprobten Horizontmächtigkeit von über $45 \mathrm{~cm}$. Zu gleichen Resultaten kommen DAVIDSON \& ACKERMAN (1993), die bei alleiniger Betrachtung des A-Horizontes eine mittlere Reduktion der Kohlenstoffvorräte von $43 \%$ konstatieren, während die Verluste unter Einbezug des B-Horizontes lediglich bei $31 \%$ liegen. Auch GUO \& GIFFORD (2002) sehen signifikante Verringerungen der C-Verluste erst ab Bodentiefen unter $30 \mathrm{~cm}$. Bei den drei zuletzt zitierten Studien handelt es sich um Reviews. 


\section{Einfluss der Bodenart}

Die Ergebnisse der untersuchten Studien zeigen einen signifikanten Zusammenhang mit dem Tongehalt. Sowohl beim organischen Kohlenstoff als auch beim Gesamtstickstoff sind die Gesamtverluste negativ mit dem Tongehalt korreliert (Tab.28). Dies deutet an, dass Mineralisierungsprozesse im Boden mit steigendem Tongehalt langsamer ablaufen.

Feinkörnige Böden haben bei gleichem Ausgangsmaterial aufgrund ihres größeren Sorptionsvermögens einen höheren Gehalt an organischer Substanz als Sand- oder Grobschluffböden (CERRI et al. 2004b, DESJARDINS et al. 2004). Dies begründet sich durch den höheren Anteil an Tonmineralen (auch Fe- und Al-Oxiden) die mit der organischen Substanz Verbindungen eingehen und stabile Aggregate im Boden erzeugen (DOMINY et al. 2002). BONDE et al. (1992) beziffern den an die Tonfraktion gebundenen SOC-Anteil in einem Oxisol in Südost-Brasilien auf mehr als $50 \%$, während auf die Sandfraktion lediglich $17 \%$ entfallen. Durch die Bindung an Tonminerale ist die organische Substanz in fein-körnigen Böden besser vor mikrobieller Zersetzung geschützt (HASSINK 1997, MCGRATH et al. 2001, SIX et al. 2002). Gleiches gilt bei Konversion in eine landwirtschaftliche Nutzung. Die höhere Aggregatstabilität feinkörniger Böden macht diese generell resistenter gegenüber mechanischer Beanspruchung (THOMAS \& AYARZA 1999), demnach ein neues Gleichgewicht zwischen Anlieferung und Abbau organischer Substanz in grobkörnigen Böden früher als in feinkörnigen erreicht wird. Bei letztgenannten verzögert sich der mikrobielle Abbau zusätzlich durch die mit zunehmenden Tongehalt häufiger auftretenden anaeroben Bedingungen (KATYAL et al. 2001).

NEUFELD et al. (2002) sehen eine langjährige konventionelle Bewirtschaftung insbesondere von Böden geringer Tongehalte wegen der schnellen und starken Reduktion des Gehaltes an organischer Substanz als hochgradig degradierende Form der Bodenbearbeitung in tropischen und subtropischen Breiten. Auch BRONICK \& LAL (2005) bescheinigen speziell Alfisolen eine höhere Gefährdung, da in diesen die organische Substanz den wesentlichen Anteil an der Bildung von Aggregaten im Boden hat. BondE et al. (1992) ermittelten, dass über 80 \% des an die Sandfraktion gebundenen SOC-Gehaltes bereits nach 12 Jahren konventionellen Anbaus mineralisiert ist, indes der Anteil in der Tonfraktion im gleichen Zeitraum lediglich $40 \%$ beträgt. Auch SHANG \& THIESSEN (2000) kommen für den gleichen Nutzungszeitraum auf einem sandigen Lehm in Nordostbrasilen auf eine relative Größenordnung von $54 \%$ in der Sandfraktion und $23 \%$ in der Tonfraktion.

Beim Gesamtstickstoff liegen die relativen Mineralisationsraten in den Korngrößenfraktionen in der gleichen Größenordnung wie die des organischen Kohlenstoffs (DALAL \& MEYER 1986b, LOBE et al. 2001). 
Tab. 29: Relative SOC- und $\mathrm{N}_{\mathrm{t}}$-Verluste in Abhängigkeit von Nutzungsdauer und Tongehalt (Oberboden)

\begin{tabular}{|c|c|c|c|c|c|c|c|c|c|}
\hline & & \multicolumn{4}{|c|}{ SOC } & \multicolumn{4}{|c|}{$\mathbf{N}_{\mathbf{t}}$} \\
\hline & & \multicolumn{2}{|c|}{ Gehalt $[\%]$} & \multicolumn{2}{|c|}{ Vorrat $[\%]$} & \multicolumn{2}{|c|}{ Gehalt $[\%]$} & \multicolumn{2}{|c|}{ Vorrat [\%] } \\
\hline & & gesamt & pro Jahr & gesamt & pro Jahr & gesamt & pro Jahr & gesamt & pro Jahr \\
\hline \multicolumn{10}{|c|}{ a. Tongehalt: $<27$ [Gew.-\%] } \\
\hline \multirow[t]{3}{*}{$<10 \mathrm{a}$} & MW & 44,3 & 8,8 & 38,2 & 7,8 & 41,0 & 7,5 & 36,6 & 6,7 \\
\hline & Stabw & 12,4 & 5,3 & 10,6 & 5,0 & 10,9 & 4,1 & 11,7 & 3,9 \\
\hline & $n$ & 22 & 22 & 20 & 20 & 16 & 16 & 16 & 16 \\
\hline \multirow[t]{3}{*}{$10-20 \mathrm{a}$} & MW & 44,5 & 3,0 & 39,3 & 2,6 & 45,3 & 3,0 & 40,7 & 2,7 \\
\hline & Stabw & 14,5 & 1,1 & 13,7 & 1,1 & 10,6 & 0,8 & 10,7 & 0,7 \\
\hline & $n$ & 14 & 14 & 16 & 16 & 10 & 10 & 10 & 10 \\
\hline \multirow[t]{3}{*}{$>20 \mathrm{a}$} & MW & 56,2 & 1,8 & 51,5 & 1,6 & 52,1 & 1,7 & 48,2 & 1,5 \\
\hline & Stabw & 9,4 & 0,5 & 9,6 & 0,4 & 7,3 & 0,5 & 8,0 & 0,5 \\
\hline & $n$ & 21 & 21 & 21 & 21 & 16 & 16 & 16 & 16 \\
\hline \multicolumn{10}{|c|}{ b. Tongehalt: > 27 [Gew.-\%] } \\
\hline \multirow[t]{3}{*}{$<10 \mathrm{a}$} & MW & 27,3 & 5,8 & 21,8 & 4,9 & 27,4 & 6,8 & 24,3 & 6,0 \\
\hline & Stabw & 15,5 & 3,3 & 14,1 & 2,8 & 15,4 & 2,8 & 14,3 & 2,6 \\
\hline & $n$ & 11 & 11 & 10 & 10 & 7 & 7 & 7 & 7 \\
\hline \multirow[t]{3}{*}{$10-20 \mathrm{a}$} & $\mathrm{x}$ & 38,5 & 2,5 & 37,8 & 2,5 & 35,8 & 2,3 & 34,6 & 2,2 \\
\hline & Stabw & 13,5 & 0,9 & 13,4 & 1,0 & 10,7 & 0,6 & 13,3 & 0,9 \\
\hline & $n$ & 11 & 11 & 15 & 15 & 8 & 8 & 9 & 9 \\
\hline \multirow[t]{3}{*}{$>20 \mathrm{a}$} & $\mathrm{x}$ & 55,6 & 1,5 & 45,8 & 1,3 & 47,7 & 1,4 & 43,1 & 1,0 \\
\hline & Stabw & 14,7 & 0,5 & 10,9 & 0,4 & 14,1 & 0,6 & 9,3 & 0,3 \\
\hline & $n$ & 7 & 7 & 14 & 14 & 5 & 5 & 3 & 3 \\
\hline \multicolumn{10}{|c|}{ Differenz (a-b) } \\
\hline$<10 \mathrm{a}$ & $\Delta[\%]$ & 17,0 & 3,0 & 16,4 & 2,9 & 13,6 & 0,7 & 12,3 & 0,7 \\
\hline $10-20 \mathrm{a}$ & $\Delta[\%]$ & 6,0 & 0,5 & 1,5 & 0,1 & 9,5 & 0,7 & 6,1 & 0,5 \\
\hline$>20 \mathrm{a}$ & $\Delta[\%]$ & 0,6 & 0,3 & 5,7 & 0,3 & 4,4 & 0,3 & 5,1 & 0,5 \\
\hline
\end{tabular}

Anm.: Zur Gewährleistung einer ausreichenden statistischen Datenbasis für die 3 Nutzungsperioden musste eine pragmatische Unterteilung der Bodenarten vorgenommen werden. Für die Unterteilung tonarmer und tonreicher Böden wurde eine eine feste Grenze bei 27 Gew.-\% Ton gesetzt. Dieser Wert bildet nach der US Soil Taxonomy (SOIL SuRvey STAFF 2003) die Untergrenze toniger (CL) und schluffig-toniger Lehme (SICL) und korrespondiert in etwa mit der Untergrenze sandig-toniger und schwach toniger Lehme (25 Gew.-\%) der Bodenkundlichen Kartieranleitung (AG BODEN 1994). Dieser Einteilung liegt der Sachverhalt zugrunde, dass der SOM-Gehalt bei gleichen Ausgangsbedingungen in tonreichen Böden generell höher ist als in tonarmen (z.B. NEUFELDT et al. 2002). Natürlich können auch feinschluffreiche Böden hohe SOM-Gehalte aufweisen, da diese aber z.T. über die gewählte Grenze abgedeckt sind und zudem in kaum einer Studie Angaben der Korngrößenunterfraktionen vorlagen, wird bei dem angewendeten Verfahren von einer plausiblen Unterteilung ausgegangen.

Zusammenfassend lässt sich festhalten, dass unter mechanischer Bodenbearbeitung in allen Korngrößenfraktionen eine Abnahme des SOM-Gehaltes zu verzeichnen ist, die zu einem großen Teil auf dem Aufbruch von Bodenaggregaten und der gleichzeitigen Freisetzung und Mineralisierung organischer Substanz beruht. Unter sandigen Bodenarten mit geringem Tonanteil verläuft der Prozess aufgrund des schwachen inneren Zusammenhaltes der Aggregate vergleichsweise schneller als unter tonreicheren Bodenarten, weshalb insbesondere diese Böden in tropischen und subtropischen Breiten unter konventioneller Bewirtschaftung relativ schnell ihren Gehalt an organischer Substanz und somit auch einen wesentlichen Nährstoffpool verlieren. In den ersten 10 Anbaujahren können die relativen Verluste der SOC- bzw. $\mathrm{N}_{\mathrm{t}^{-}}$ Vorräte tonarmer Böden im Mittel 16 \% bzw. 12 \% höher ausfallen als auf tonreichen Böden (Tab.29). Mit zunehmender Kultivierungsdauer verringern sich die Unterschiede. Nach mehr 
als 20-jähriger kontinuierlicher Nutzung liegt die mittlere Abweichung der SOC- und $\mathrm{N}_{\mathrm{t}^{-}}$ Verluste im Vergleich tonarmer und tonreicher Böden bei etwa $5 \%$.
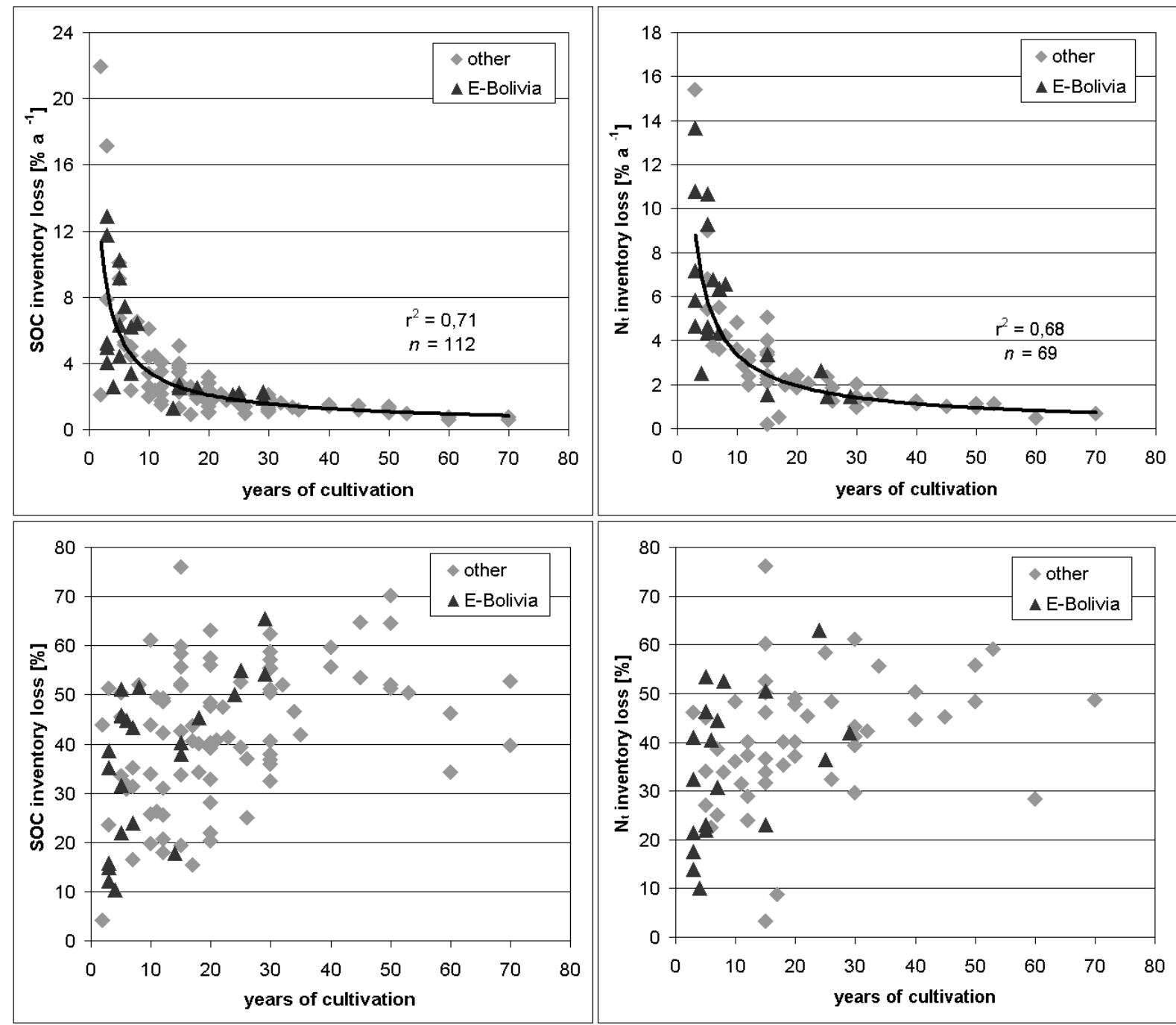

Abb. 21: relative SOC- und $\mathrm{N}_{\mathrm{t}}$-Verluste (Vorräte) in Abhängigkeit von der Nutzungsdauer (Oberboden)

\section{Zeitliche Änderung}

Sowohl die Gesamtverluste als auch die jährlichen Raten sind signifikant mit der Nutzungsdauer korreliert (Tab.28). Während die Gesamtverluste von SOC und $\mathrm{N}_{t}$ in Abhängigkeit von der Zeit unterschiedlich hoch ausfallen (Abb.21), zeigt sich bei den jährlichen Abnahmeraten ein eindeutiger Trend mit hohen initialen Verlusten und einem in der Folge langsameren Rückgang (Abb.21). Nach 20 Jahren kontinuierlicher Nutzung reduzieren sich die SOC- und $\mathrm{N}_{\mathrm{t}}$-Vorräte im Oberboden im Mittel um 35-40 \%, wovon der weitaus größte Anteil auf die ersten 10 Anbaujahre entfällt. In diesem Zeitraum liegt die jährliche Verlustrate sowohl für SOC als auch für $\mathrm{N}_{\mathrm{t}}$ im Mittel zwischen 5-8 \%. Die Rate verringert sich konstant auf 2,5\% nach mehr als 10 Jahren und auf 1,5\% nach mehr als 20 Jahren ackerbaulicher Nutzung. Die Gesamtverluste der SOC- und $\mathrm{N}_{\mathrm{t}}$-Vorräte in Oberböden tropischer und sub- 
tropischer Breiten liegen bei einer kontinuierlichen konventionellen Nutzung von mehr als 20 Jahren im Mittel zwischen 46-52 \% (SOC) bzw. 43-48 \% $\left(\mathrm{N}_{\mathrm{t}}\right)$.

Die in Tabelle 29 dargestellten Verluste fließen als Größenordnung in die weitere Auswertung ein. Die abgeleiteten SOC-Verluste bewegen sich in einer ähnlichen Größenordnung mit denen in den Reviews von MURTY et al. (2002), DAVIDSON \& ACKERMANN (1993) und GUO \& GIFFORD (2002) ermittelten Werten, die nach langjähriger Anbaunutzung mittlere Verluste der SOC-Vorräte im Oberboden von $45 \%$, $43 \%$ bzw. 50 \% ermittelten. Im Unterschied zu vorliegender Arbeit wurden bei deren Analysen z.T. auch Studien außertropischer Länder einbezogen. Die abgeleiteten Verluste stellen aufgrund der räumlichen Variabilität der Bodeneigenschaften relative Größenordnungen dar. Die an den Kontrollflächen (natürliche Vegetation) erhobenen Ausgangsgehalte stellen nicht zwangsläufig den realen Zustand einer Ackerfläche vor deren Inkulturnahme dar. Der Fehler erhöht sich mit zunehmendem Abstand zur Kontrollfläche, bzw. je mehr Nutzflächen sich auf eine Kontrollfläche beziehen. Über Unsicherheiten des Vergleichs von Daten verschiedener Studien, siehe z.B. BERNOUX et al. (2002) und MurTy et al. (2002).

\subsubsection{Ableitung initialer SOC- und $\mathrm{N}_{\mathrm{t}}$-Gehalte im Untersuchungsgebiet}

$\mathrm{Da}$ weder SOC- noch $\mathrm{N}_{\mathrm{t}}$-Gehalte der Oberböden zuverlässig regionalisierbar waren (Kap.5.1.1), wurden aus den Bodendaten des Untersuchungsgebiets Mittelwerte abgeleitet, die näherungsweise das Ausgangsniveau unter natürlicher Vegetation repräsentieren. In Abbildung 22 sind die jeweiligen Oberbodengehalte für die verschiedenen Regionen des Untersuchungsgebiets dargestellt. Die z.T. großen Spannweiten sind Folge kleinräumiger Wechsel von Bodeneigenschaften (Kap.2.2.2, 5.1.1), die über den Regionalisierungsansatz nicht adäquat nachvollzogen werden konnten. Inwieweit in diesem Zusammenhang auch Messfehler eine Rolle spielen, kann nicht beurteilt werden. Bezogen auf das gesamte Untersuchungsgebiet konzentriert sich die Hälfte aller Werte im Bereich von 1-2 $\mathrm{g} \mathrm{kg}^{-1}$ beim organischen Kohlenstoff bzw. 0,12-0,23 $\mathrm{g} \mathrm{kg}^{-1}$ beim Gesamtstickstoff. Damit sind die Böden nach Richtwerten von CIAT (Tab.A1, Anhang) in ihrem natürlichen Zustand mittel bis gut mit Stickstoff versorgt, während sich die Gehalte an organischem Kohlenstoff auf niedrigem bis mittlerem Niveau befinden. Da innerhalb der Alluvialebene die Regionen nicht klar voneinander abgrenzbar sind und wegen des z.T. geringen Stichprobenumfangs der verschiedenen Bodenarten wurden für das Untersuchungsgebiet mittlere Ausgangsniveaus gemäß der Differenzierung nach dem Tongehalt festgelegt (Kap.5.1.2.1). Erkennbar ist, dass tonreiche Böden höhere Gehalte an organischer Substanz aufweisen als tonarme (Abb.23) ${ }^{26}$.

\footnotetext{
${ }^{26}$ Die Angabe "tonarme Böden" umfasst im Untersuchungsgebiet überwiegend die Bodenart sandige Lehme (SL) sowie weniger Häufig Lehme (L) und schluffige Lehme (SIL). Tonreiche Böden beziehen sich mehrheitlich auf schluffig-tonige (SICL) und tonige Lehme (CL) sowie z.T. auf reine Tonböden (C) und schluffige Tone (SIC).
} 
Regional umfasst dies mehrheitlich die Böden der Alluvialebene, mit Ausnahme des südwestlichen Bereichs. Innerhalb der Alluvialebene sind dort die Böden mit den höchsten Sandanteilen entwickelt (Abb.4, Abb.20). Böden hoher Sandgehalte finden sich zudem im subandinen Bereich und auf dem brasilianischen Schild, diese aber spielen in ackerbaulicher Hinsicht keine nennenswerte Rolle im Untersuchungsgebiet.
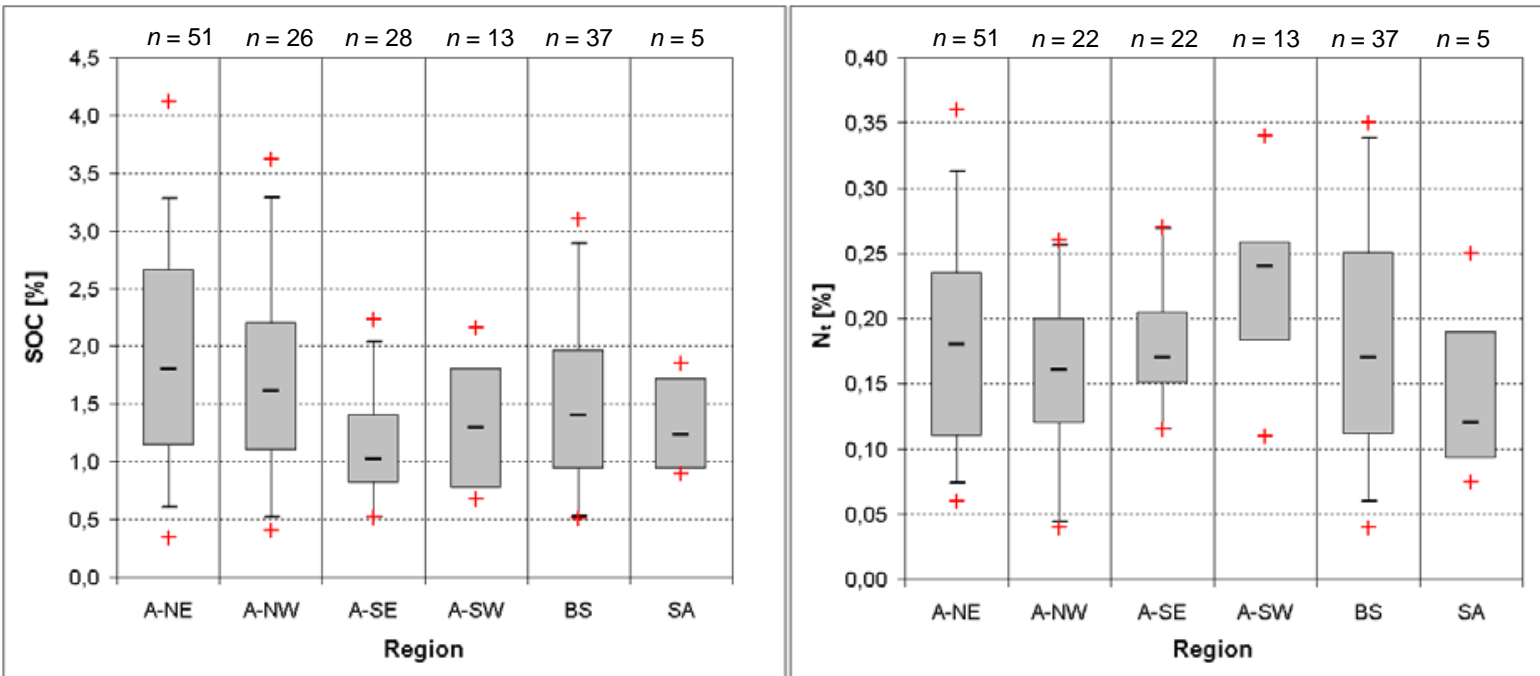

Abb. 22: Boxplots der SOC- und $\mathrm{N}_{\mathrm{t}}$-Ausgangsgehalte nach Regionen

A: Alluvialebene (NE: Nordost, NW: Nordwest, SE: Südost, SW: Südwest), BS: brasilianischer Schild, SA: Subandin
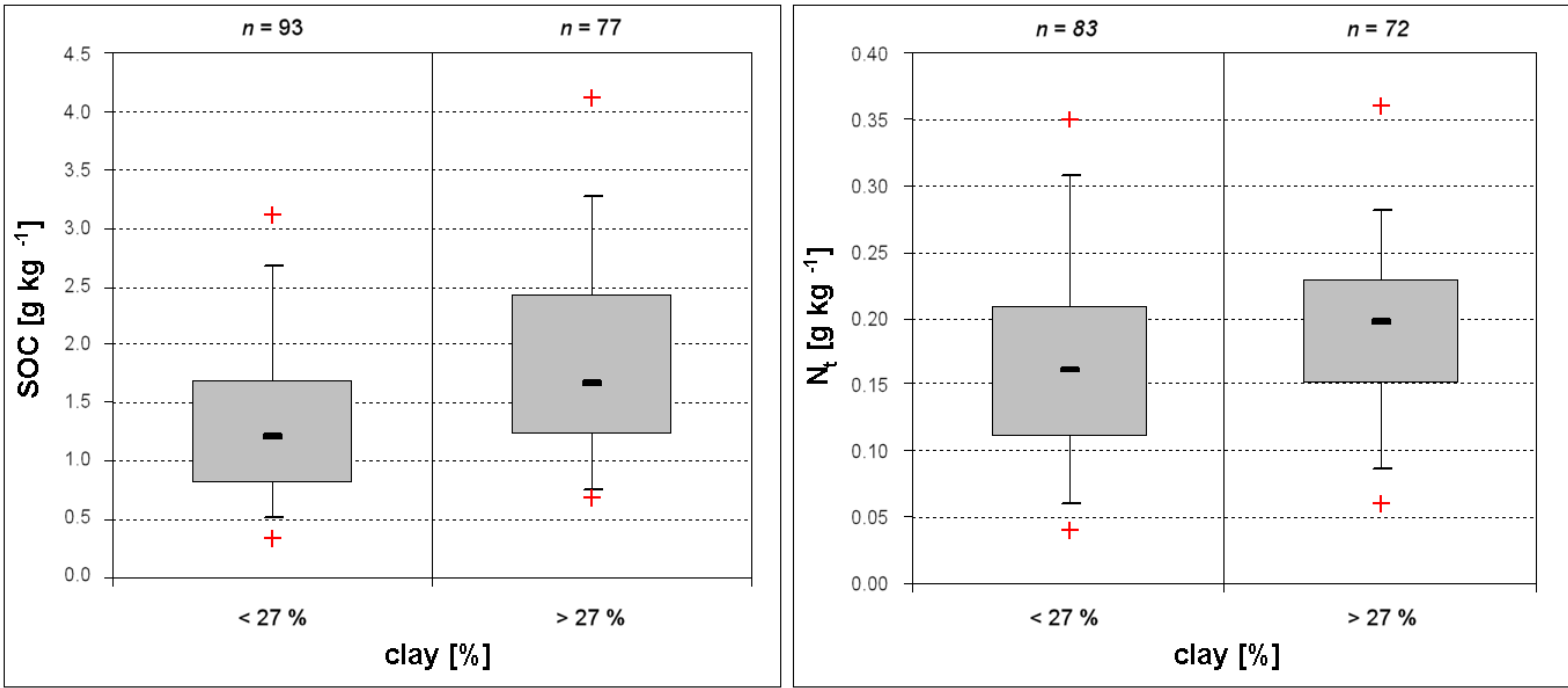

Abb. 23: Boxplots der SOC- und $\mathrm{N}_{\mathrm{t}}$-Ausgangsgehalte in Abhängigkeit vom Tongehalt

Aus den Datensätzen wurden Ausreißer entfernt und das jeweilige arithmetische Mittel $( \pm$ Stabw) tonarmer- bzw. tonreicher Böden als Ausgangsniveau der SOC- und $\mathrm{N}_{\mathrm{t}}$-Gehalte festgelegt (Tab.30). Für tonarme Böden ergeben sich Gehalte von 1,28 $\pm 0,53 \mathrm{~g} \mathrm{~kg}^{-1}$ (SOC) bzw. 0,17 $\pm 0,06 \mathrm{~g} \mathrm{~kg}^{-1}\left(\mathrm{~N}_{\mathrm{t}}\right.$ und für Böden höherer Tongehalte 1,82 $\pm 0,67 \mathrm{~g} \mathrm{~kg}^{-1}$ (SOC) bzw. $0,19 \pm 0,05 \mathrm{~g} \mathrm{~kg}^{-1}\left(\mathrm{~N}_{\mathrm{t}}\right.$. Für den organischen Kohlenstoff errechnen sich aus den Gehalten bis 
zu einer mittleren Bodentiefe von $20 \mathrm{~cm}$ überschlägig Vorräte von $32 \pm 13 \mathrm{tha}^{-1}$ für tonarme Böden und $46 \pm 17 \mathrm{t} \mathrm{ha}^{-1}$ für tonreiche Böden und für Gesamtstickstoff von 4,2 $\pm 1,6 \mathrm{t} \mathrm{ha}^{-1}$ bzw. 4,8 $\pm 1,3 \mathrm{t} \mathrm{ha}^{-1}$. Die den Vorratsberechnungen zugrunde liegenden Lagerungsdichten $\left(\mathrm{d}_{\mathrm{b}}\right)$ sind arithmetische Mittelwerte von Messungen, die unter natürlicher Vegetation im Untersuchungsgebiet durchgeführt wurden (Kap.5.1.3).

Die aus den Bodendaten abgeleiteten SOM-Vorräte sind vergleichbar mit denen von BARBER (1995) auf sandigen Lehmen der Alluvialebene ermittelten Werten. Seinen Angaben zufolge liegen die natürlichen Humusvorräte in einer Größenordnung von 59-101 t ha ${ }^{-1}(0-30$ $\mathrm{cm}$ ). Bei Umrechnung der in Tabelle 30 angegebenen SOM-Gehalte bis zu einer Bodentiefe von $30 \mathrm{~cm}$ ergeben sich mittlere Vorräte von $84 \mathrm{t} \mathrm{ha}^{-1}$ für tonarme und $118 \mathrm{t} \mathrm{ha}^{-1}$ für tonreiche Böden. Natürlich schwanken die Werte, wie die Ergebnisse von BARBER (1995) belegen, aufgrund der kleinräumigen Heterogenität der Bodeneigenschaften z.T. beträchtlich, dennoch lassen sich über die Annahme eines einheitlichen Ausgangsniveaus vertretbare Tendenzen aufzeigen.

Tab. 30: Mittlerer SOC-, SOM- und $\mathrm{N}_{\mathrm{t}}$-Status im Oberboden unter natürlicher Vegetation in Abhängigkeit vom Tongehalt

\begin{tabular}{|c|c|c|c|c|c|c|c|c|c|}
\hline & $\begin{array}{l}\text { Tiefe } \\
{[\mathrm{cm}]}\end{array}$ & $\begin{array}{c}\text { SOC } \\
{\left[\mathrm{g} \mathrm{kg}^{-1}\right]}\end{array}$ & $\underset{\left[\mathrm{g} \mathrm{kg}^{-1}\right]}{\mathbf{S O M}}$ & $\begin{array}{l}{ }^{+} \mathbf{S O C} \\
{\left[\mathrm{t} \mathrm{ha}^{-1}\right]}\end{array}$ & $\begin{array}{l}{ }^{+} \text {SOM } \\
{\left[\mathrm{t} \mathrm{ha}^{-1}\right]}\end{array}$ & $\begin{array}{l}\text { Tiefe } \\
{[\mathrm{cm}]}\end{array}$ & $\begin{array}{c}\mathbf{N}_{\mathbf{t}} \\
{\left[\mathrm{g} \mathrm{kg}^{-1}\right]}\end{array}$ & $\begin{array}{c}{ }^{+} \mathbf{N}_{\mathbf{t}} \\
{\left[\mathrm{t} \mathrm{ha}^{-1}\right]}\end{array}$ & $\begin{array}{c}\mathbf{d}_{\mathbf{b}} \\
{\left[\mathrm{g} \mathrm{cm}^{-3}\right]}\end{array}$ \\
\hline \multicolumn{10}{|c|}{ a. Tongehalt: $<27$ [Gew.-\%] } \\
\hline Min & 6 & 0,52 & 0,90 & * & $*$ & 6 & 0,07 & $*$ & 0,94 \\
\hline Max & 44 & 2,67 & 4,60 & $*$ & $*$ & 44 & 0,30 & $*$ & 1,56 \\
\hline MW & 21 & 1,28 & 2,21 & 32,4 & 55,8 & 21 & 0,17 & 4,2 & 1,26 \\
\hline Stabw & 8 & 0,53 & 0,91 & $*$ & $*$ & 9 & 0,06 & $*$ & 0,14 \\
\hline$n$ & 85 & 85 & 85 & $*$ & $*$ & 75 & 75 & $*$ & 48 \\
\hline \multicolumn{10}{|c|}{ b. Tongehalt: > 27 [Gew.-\%] } \\
\hline Min & 7 & 0,75 & 1,29 & $*$ & $*$ & 7 & 0,09 & $*$ & 0,91 \\
\hline Max & 42 & 3,27 & 5,64 & * & $*$ & 42 & 0,27 & $*$ & 1,49 \\
\hline MW & 24 & 1,82 & 3,14 & 45,7 & 78,8 & 24 & 0,19 & 4,8 & 1,25 \\
\hline Stabw & 7 & 0,67 & 1,16 & $*$ & $*$ & 8 & 0,05 & $*$ & 0,15 \\
\hline$n$ & 71 & 71 & 71 & $*$ & $*$ & 66 & 66 & $*$ & 23 \\
\hline
\end{tabular}

\subsubsection{SOC- und $\mathrm{N}_{\mathrm{t}}$-Verluste im Untersuchungsgebiet - regionale Analyse}

Auf Grundlage der mittleren SOC- und $\mathrm{N}_{\mathrm{t}}$-Gehalte und der daraus abgeleiteten Bodenvorräte unter natürlicher Vegetation im Untersuchungsgebiet (Tab.30) errechnen sich über die relativen Verluste (Tab.29) die mittleren Nährelementzustände der verschiedenen Nutzungszeiträume (Tab.31). Nach Konversion der natürlichen Vegetation in Ackerflächen sind auf tonarmen Böden die höchsten relativen SOC- und $\mathrm{N}_{\mathrm{t}}$-Verluste zu verzeichnen (Tab.31). Bei diesen handelt es sich überwiegend um sandige Lehme, die sich in der Alluvialebene in relativer Nähe zur Andenfußzone konzentrieren. Mit zunehmender Entfernung vom Anden- 
rand steigt tendenziell der Tonanteil und damit die Kohlenstoff- und Stickstoffvorräte in den Böden. Generell ist die Nährstoffnachlieferung bei tonarmen Böden, sofern keine Dünger appliziert werden, bereits innerhalb der ersten 10 Anbaujahre kritisch zu beurteilen, wogegen die Gehalte der tonreichen Böden erst nach mehr als 20 Jahren kontinuierlicher Nutzung auf einem ähnlich niedrigen Niveau sind. Zwar können die absoluten SOC-Verluste tonreicher Böden die tonarmer übersteigen, dennoch befinden sich die SOC-Vorräte, aufgrund der höheren Ausgangsgehalte, auch nach einer Kultivierungsdauer von bis zu 20 Jahren z.T. noch auf dem Niveau der Ausgangsgehalte tonarmer Substrate. Nach langjähriger Ackernutzung liegen die Bodenvorräte der tonreichen Böden im Mittel $9 \mathrm{t} \mathrm{ha}^{-1}$ (SOC) bzw. 0,6 t ha ${ }^{-1}\left(\mathrm{~N}_{\mathrm{t}}\right)$ über denen der tonarmen, was allgemein deren bessere Nährstoffsituation dokumentiert. Im Durchschnitt ist nach langjähriger kontinuierlicher Nutzung von Verlusten in einer Größenordnung von 17-21 $\mathrm{t} \mathrm{ha}^{-1}$ (SOC) bzw. $2 \mathrm{tha}^{-1}\left(\mathrm{~N}_{\mathrm{t}}\right)$ auszugehen.

Tab. 31: Mittlere SOC-Verluste im Oberboden in Abhängigkeit von Nutzungsdauer und Tongehalt

\begin{tabular}{|c|c|c|c|c|c|c|}
\hline \multirow{3}{*}{$\begin{array}{l}\text { SOC } \\
\text { Nutzungsperiode }\end{array}$} & \multicolumn{3}{|c|}{ Tongehalt: $<27$ [Gew.-\%] } & \multicolumn{3}{|c|}{ Tongehalt: > 27 [Gew.-\%] } \\
\hline & \multirow[t]{2}{*}{ Niveau } & \multicolumn{2}{|c|}{ Verlust } & \multirow[t]{2}{*}{ Niveau } & \multicolumn{2}{|c|}{ Verlust } \\
\hline & & absolut & relativ & & absolut & relativ \\
\hline Gehalt & {$\left[\mathrm{g} \mathrm{kg}^{-1}\right]$} & {$\left[\mathrm{g} \mathrm{kg}^{-1}\right]$} & $\%$ & {$\left[\mathrm{~g} \mathrm{~kg}^{-1}\right]$} & {$\left[\mathrm{g} \mathrm{kg}^{-1}\right]$} & $\%$ \\
\hline natürliche Vegetation & $1,28 \pm 0,53$ & $*$ & $*$ & $1,82 \pm 0,67$ & $*$ & $*$ \\
\hline$<10 \mathrm{a}$ & 0,71 & $0,57 \pm 0,16$ & $44,3 \pm 12,4$ & 1,32 & $0,50 \pm 0,28$ & $27,3 \pm 15,5$ \\
\hline $10-20 \mathrm{a}$ & 0,71 & $0,57 \pm 0,19$ & $44,5 \pm 14,5$ & 1,12 & $0,70 \pm 0,25$ & $38,5 \pm 13,5$ \\
\hline$>20 a$ & 0,56 & $0,72 \pm 0,12$ & $56,2 \pm 9,4$ & 0,81 & $1,01 \pm 0,27$ & $55,6 \pm 14,7$ \\
\hline Vorrat $(0-20 \mathrm{~cm})$ & {$\left[\mathrm{t} \mathrm{ha}^{-1}\right]$} & {$\left[\mathrm{tha}^{-1}\right]$} & $\%$ & {$\left[\mathrm{tha}^{-1}\right]$} & {$\left[\mathrm{t} \mathrm{ha}^{-1}\right]$} & $\%$ \\
\hline natürliche Vegetation & $32,4 \pm 13,4$ & $*$ & $*$ & $45,7 \pm 16,9$ & $*$ & $*$ \\
\hline$<10 \mathrm{a}$ & 20,6 & $12,4 \pm 3,4$ & $38,2 \pm 10,6$ & 36,8 & $10,0 \pm 6,4$ & $21,8 \pm 14,1$ \\
\hline $10-20 \mathrm{a}$ & 20,2 & $12,7 \pm 4,4$ & $39,3 \pm 13,7$ & 29,3 & $17,3 \pm 6,1$ & $37,8 \pm 13,4$ \\
\hline$>20 \mathrm{a}$ & 16,2 & $16,7 \pm 3,1$ & $51,5 \pm 9,6$ & 25,5 & $20,9 \pm 5,0$ & $45,8 \pm 10,9$ \\
\hline
\end{tabular}

Tab. 32: Mittlere $\mathbf{N}_{\mathbf{t}}$-Verluste im Oberboden Abhängigkeit von Nutzungsdauer und Tongehalt

\begin{tabular}{|c|c|c|c|c|c|c|}
\hline \multirow{3}{*}{$\begin{array}{l}\mathbf{N}_{\mathbf{t}} \\
\text { Nutzungsperiode }\end{array}$} & \multicolumn{3}{|c|}{ Tongehalt: < 27 [Gew.-\%] } & \multicolumn{3}{|c|}{ Tongehalt: > 27 [Gew.-\%] } \\
\hline & \multirow[t]{2}{*}{ Niveau } & \multicolumn{2}{|c|}{ Verlust } & \multirow[t]{2}{*}{ Niveau } & \multicolumn{2}{|c|}{ Verlust } \\
\hline & & absolut & relativ & & absolut & relativ \\
\hline Gehalt & {$\left[\mathrm{g} \mathrm{kg}^{-1}\right]$} & {$\left[\mathrm{g} \mathrm{kg}^{-1}\right]$} & $\%$ & {$\left[\mathrm{~g} \mathrm{~kg}^{-1}\right]$} & {$\left[\mathrm{g} \mathrm{kg}^{-1}\right]$} & $\%$ \\
\hline natürliche Vegetation & $0,17 \pm 0,06$ & $*$ & $*$ & $0,19 \pm 0,05$ & $*$ & $*$ \\
\hline$<10$ a & 0,10 & $0,07 \pm 0,02$ & $41,0 \pm 10,9$ & 0,14 & $0,05 \pm 0,03$ & $27,4 \pm 15,4$ \\
\hline $10-20 \mathrm{a}$ & 0,09 & $0,08 \pm 0,02$ & $45,3 \pm 10,6$ & 0,12 & $0,07 \pm 0,02$ & $35,8 \pm 10,7$ \\
\hline$>20 a$ & 0,08 & $0,09 \pm 0,01$ & $52,1 \pm 7,3$ & 0,10 & $0,09 \pm 0,03$ & $47,7 \pm 14,1$ \\
\hline Vorrat $(0-20 \mathrm{~cm})$ & {$\left[\mathrm{t} \mathrm{ha}^{-1}\right]$} & {$\left[\mathrm{t} \mathrm{ha}^{-1}\right]$} & $\%$ & {$\left[\mathrm{t} \mathrm{ha}^{-1}\right]$} & {$\left[\mathrm{t} \mathrm{ha}^{-1}\right]$} & $\%$ \\
\hline natürliche Vegetation & $4,2 \pm 1,6$ & $*$ & $*$ & $4,8 \pm 1,3$ & $*$ & * \\
\hline$<10$ a & 2,7 & $1,5 \pm 0,5$ & $36,6 \pm 11,7$ & 3,7 & $1,2 \pm 0,7$ & $24,3 \pm 14,3$ \\
\hline $10-20 \mathrm{a}$ & 2,5 & $1,7 \pm 0,4$ & $40,7 \pm 10,7$ & 3,2 & $1,7 \pm 0,6$ & $34,6 \pm 13,3$ \\
\hline$>20 \mathrm{a}$ & 2,2 & $2,0 \pm 0,3$ & $48,2 \pm 8,0$ & 2,8 & $2,1 \pm 0,4$ & $43,1 \pm 9,3$ \\
\hline
\end{tabular}


Die Werte der Tabellen 31 und 32 bilden die Grundlage für die räumliche Schätzung der durch Kultivierung induzierten SOC- bzw. $\mathrm{N}_{\mathrm{t}}$-Verluste. Dabei wird der regionalisierte Tongehalt der Böden (Kap.5.1.1) in Bezug zur Bewirtschaftungsperiode gesetzt (Kap.4.2.5.1) und über die mittleren Verluste (Kap.5.1.2.1) die Größenordnung der Oberbodenvorräte 2001 bestimmt (Abb.23). Zwischen den verschiedenen Landnutzungssystemen bestehen in Bezug auf die Höhe der Verluste vermeintlich kaum Abweichungen (Kap.5.1.2). Zu vermuten wäre, dass die Reduktion der Vorräte in den Gebieten der Kleinbauern durch eine höhere Produktdiversifizierung und eine stärkere Rotation der Kulturen langsamer verläuft, was aber mangels Untersuchungen nicht quantifizierbar ist. Unklar bleibt auch, ob die Entwicklung in Gebieten mit zwei Anbauzyklen pro Jahr schneller verläuft. Allgemein wird Bodenbearbeitung, insbesondere durch Pflügen, als einer der Hauptfaktoren abnehmender SOC- und $\mathrm{N}_{\mathrm{t}}$-Vorräte in Ackerböden angesehen (REICOSKY 1997). Nach BARBER et al. (1996) wird der Boden in diesen Gebieten bis zu 10 mal im Jahr mechanisch bearbeitet. Welchen Einfluss das allerdings in zeitlicher Hinsicht auf die Verluste hat, kann nicht quantifiziert werden, da Analysen dazu fehlen. Wahrscheinlich aber wird durch die wiederholte, intensive Bodenbearbeitung, die Mineralisierung der organischen Bodensubstanz in diesen Gebieten zusätzlich beschleunigt.

Tab. 33: Mittlere Gehalte und Vorräte organischer Substanz (SOM) der Anbauflächen 2001

\begin{tabular}{cccccc}
\hline Nutzungszeitraum & Tongehalt & $\begin{array}{c}\text { Vorräte }\left(\mathrm{t} \mathrm{ha}^{-1}, 0-20 \mathrm{~cm}\right) \\
\text { mittlere Schwankungsbreite }\end{array}$ & MW & $\begin{array}{c}\text { Gehalte }\left(\mathrm{g} \mathrm{kg}^{-1}\right) \\
\text { mittlere Schwankungsbreite }\end{array}$ & MW \\
\hline$>20 \mathrm{a}$ & $<27 \%$ & $21,7-32,5$ & 27,1 & $0,76-1,17$ & 0,97 \\
$>20 \mathrm{a}$ & $>27 \%$ & $34,1-51,3$ & 42,7 & $0,93-1,85$ & 1,39 \\
$10-20 \mathrm{a}$ & $<27 \%$ & $26,3-41,6$ & 33,9 & $0,90-1,54$ & 1,22 \\
$10-20 \mathrm{a}$ & $>27 \%$ & $38,4-59,6$ & 49,0 & $1,51-2,35$ & 1,93 \\
$<10 \mathrm{a}$ & $<27 \%$ & $28,6-40,4$ & 34,5 & $0,96-1,50$ & 1,23 \\
$<10 \mathrm{a}$ & $>27 \%$ & $50,5-72,7$ & 61,6 & $1,79-2,77$ & 2,28 \\
\hline
\end{tabular}

Die niedrigsten SOC- und $\mathrm{N}_{\mathrm{t}}$-Vorräte sind 2001 auf den tonarmen Böden der nordwestlichen und südwestlichen Alluvialebene zu verzeichnen, die seit mehr als 20 Jahren kultiviert werden (zentrale Zone). Die Bodenvorräte liegen dort unter Annahme einer mittleren Reduktion von $52 \%$ (SOC) bzw. $48 \%\left(\mathrm{~N}_{\mathrm{t}}\right)$ bei etwa 9-22 $\mathrm{t} \mathrm{ha}^{-1}$ (SOC) bzw. 1,3-3,0 $\mathrm{t} \mathrm{ha}^{-1}$ $\left(\mathrm{N}_{\mathrm{t}}\right)$. Viele der Flächen werden aktuell extensiv beweidet, da ein ertragreicher Anbau auf diesen nicht mehr möglich ist. Bereits in den ersten 10 Jahren nach Inkulturnahme reduzieren sich die Vorräte um durchschnittlich $12 \mathrm{t} \mathrm{ha}^{-1}$ (SOC) bzw. 1,5 $\mathrm{t} \mathrm{ha}^{-1}\left(\mathrm{~N}_{\mathrm{t}}\right.$ ), woran sich in den folgenden knapp 10 Jahren nur graduelle Verluste anschließen. Im Vergleich mit langjährig bewirtschafteten Flächen auf diesen Böden liegen die Vorräte in den ersten 20 Anbaujahren noch um ca. $4 \mathrm{t} \mathrm{ha}^{-1}$ (SOC) bzw. 0,5 $\mathrm{t} \mathrm{ha}^{-1}\left(\mathrm{~N}_{\mathrm{t}}\right)$ höher. Auf einem generell höheren Niveau befinden sich die SOC- und $\mathrm{N}_{\mathrm{t}}$-Vorräte tonreicher Böden in der Alluvialebene. Selbst die am längsten genutzten Flächen weisen 2001 noch bis zu $5 \mathrm{t} \mathrm{ha}^{-1}$ höhere SOC-Vorräte auf, als die 
erst seit wenigen Jahren in Nutzung befindlichen Anbauflächen auf tonarmen Böden. Die ältesten Anbauflächen auf tonreichen Böden befinden sich in der zentralen Zone in größerer Entfernung zum Andenrand sowie zu einem geringen Teil in der Expansionszone. Allgemein liegt das Niveau auf diesen Flächen zwischen 16-34 $\mathrm{t} \mathrm{ha}^{-1}$ (SOC) bzw. 2,0-3,5 $\mathrm{t} \mathrm{ha}^{-1}\left(\mathrm{~N}_{\mathrm{t}}\right)$, während die längstenfalls seit Mitte der 1980er Jahre bewirtschafteten Flächen Werte von 18$39 \mathrm{t} \mathrm{ha}^{-1}$ (SOC) bzw. 2,3-4,0 t ha ${ }^{-1}\left(\mathrm{~N}_{\mathrm{t}}\right)$ aufweisen. Letztgenannte Bereiche befinden sich bereits zu großen Teilen in der Expansionszone. Gleichwohl das Niveau der tonreichen Böden generell über dem der tonarmen liegt, zeigt sich, dass die absoluten Verluste zwischen diesen nicht wesentlich differieren (Tab.31, 32). Allein in den ersten 10 Anbaujahren sind Humuspartikel in tonreichen Böden noch besser vor Freisetzung und Mineralisation geschützt. In diesem Zeitraum liegt die mittlere Abnahme annähernd 2,5 $\mathrm{tha}^{-1}$ (SOC) bzw. 0,3 $\mathrm{tha}^{-1}\left(\mathrm{~N}_{\mathrm{t}}\right)$ unter der sandiger Lehme. Die jüngsten Anbauflächen auf tonreichen Böden sind, mit wenigen Ausnahmen, in der Expansionszone konzentriert. Auf diesen liegen die Bodenvorräte 2001 in einer Größenordnung von 23-49 $\mathrm{t} \mathrm{ha}^{-1}$ (SOC) bzw. 2,6-4,6 t ha ${ }^{-1}\left(\mathrm{~N}_{\mathrm{t}}\right)$.

Tab. 34: Einordnung der Bodenvorräte organischer Substanz

\begin{tabular}{lc}
\hline Bezeichnung des Bodens & t ha $^{-\mathbf{1}} \mathbf{( \mathbf { 0 - 2 0 } \mathbf { c m } )}$ \\
\hline humusarm & $<30$ \\
schwach humos & $30-60$ \\
mäßig humos & $60-120$ \\
stark humos & $120-300$ \\
humusreich & $300-450$ \\
\hline & Quelle: ScHULTZ (2000)
\end{tabular}

Insgesamt variieren die Vorräte organischer Substanz (SOM) in den Oberböden $(0-20 \mathrm{~cm})$ der landwirtschaftlichen Nutzflächen im Raum Santa Cruz 2001 durchschnittlich zwischen 27-62 t ha ${ }^{-1}$ (Tab.33). Die langjährig kultivierten tonarmen Böden der zentralen Zone gelten nach SCHULTZ (2000) bereits als humusarm (Tab.34). Je nach Ausgangsgehalt trifft dies z.T. auch auf die weniger lang bewirtschafteten tonarmen Böden zu. Der größte Teil der länger in Nutzung befindlichen Ackerflächen im Untersuchungsraum kann, unabhängig von der Bodenart und der Nutzungsdauer, 2001 als schwach humos eingestuft werden. Lediglich die erst in den 1990er Jahren erschlossenen Ackerflächen in der Expansionszone weisen noch mittlere Humusvorräte auf. Die Einordnung entspricht der nach dem Interpretationsschlüssel von CIAT (Tab.A1, Anhang), dem allerdings Konzentrationsangaben für die Bewertung zugrunde liegen. Nach dem Bewertungsschema von CIAT sind die Böden unter natürlicher Vegetation generell gut mit Stickstoff versorgt, so dass in den ersten Anbaujahren, trotz hoher relativer Verluste, noch keine ausgeprägten Defizite hinsichtlich der Stickstoffversorgung zu erwarten sind. Erst nach mehr als 20-jähriger Nutzung müssen die $\mathrm{N}_{\mathrm{t}}$-Gehalte auf den tonarmen Böden kritisch bewertet werden, demgegenüber sich diese auf den tonreichen Böden noch länger 


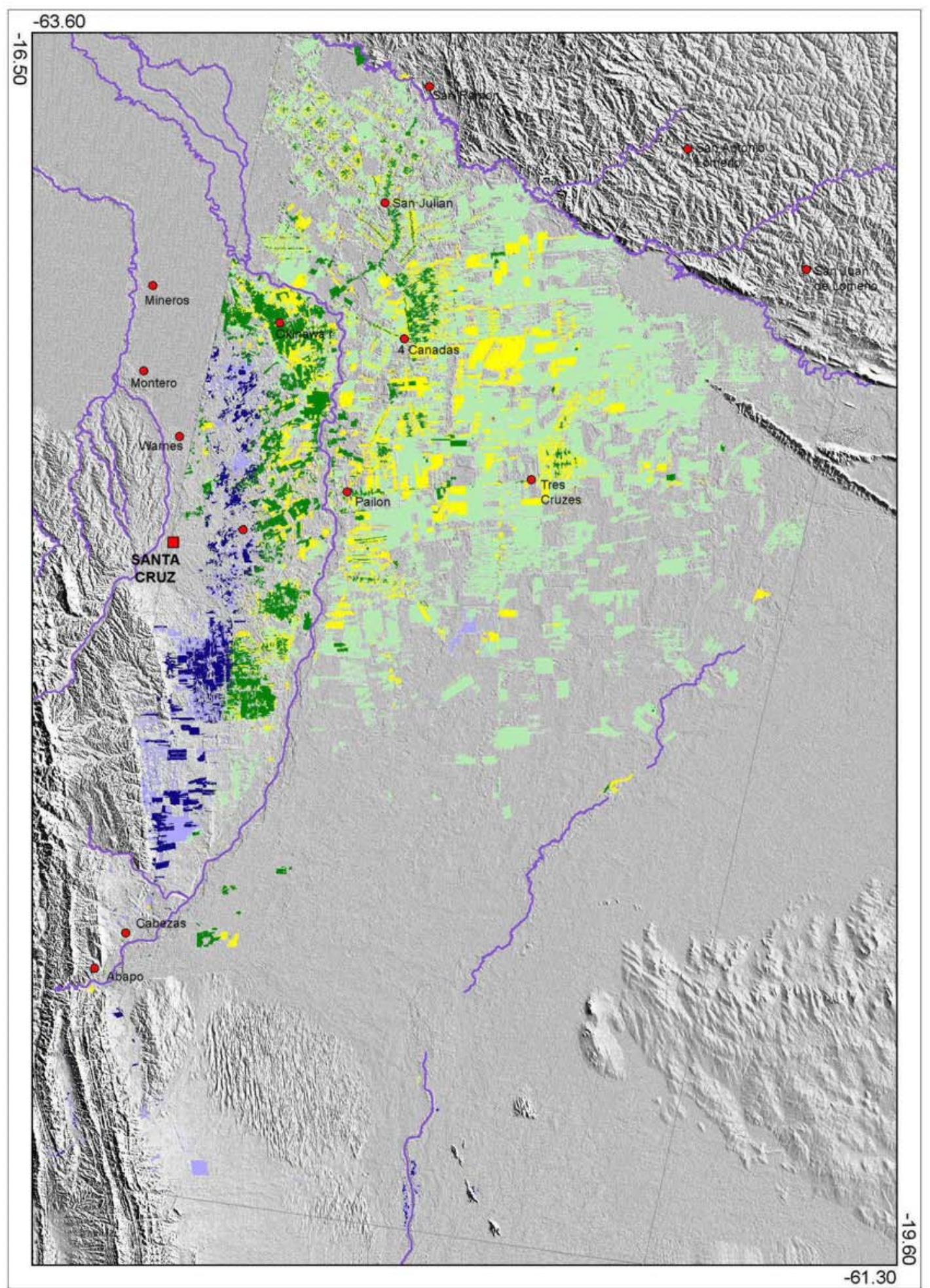

Soil carbon and nitrogen stocks under agricultural use 2001 limits of variation [t ha-1, $0-20 \mathrm{~cm}$ ]
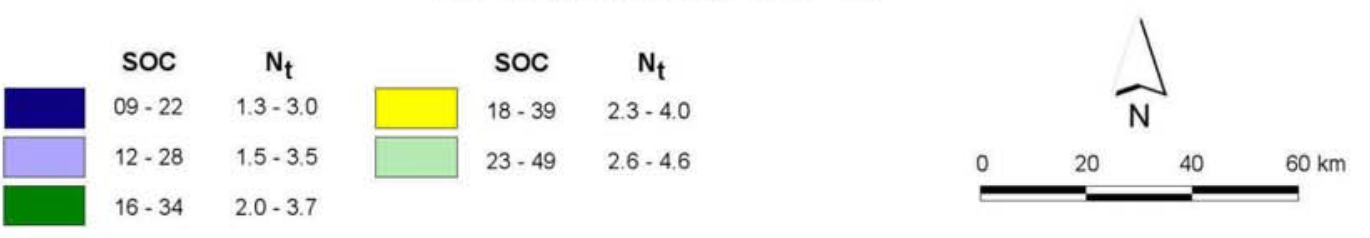

Abb. 24: Mittlere SOC- und $\mathrm{N}_{\mathrm{t}}$-Vorräte in den Oberböden von Ackerflächen 2001 
auf einem höheren Niveau befinden (Tab.32).

Den infolge langjähriger konventioneller Bearbeitung nährstoffverarmten tonarmen Böden, stehen die aufgrund höherer Gehalte an organischer Substanz besser mit Nährstoffen versorgten tonreichen Böden gegenüber. Diese können bezüglich ihrer chemischen Bodeneigenschaften und der meist kürzeren Nutzungsdauer (Expansionszone) noch länger profitabel bewirtschaftet werden. Allerdings führen auch auf diesen Böden intensive Bearbeitungsprozesse zu einer schnellen Mineralisation der organischen Bodensubstanz. Da über die derzeit praktizierten Bewirtschaftungsweisen keine SOM-Anreicherung im Boden erfolgt, ist mittelfristig mit spürbaren Nährstoffverlusten und einer abnehmenden Ertragsfähigkeit der Böden zu rechnen. Aus diesen Gründen sollten Strategien überlegt werden, die Humusgehalte auf einem höheren Niveau zu halten, um längerfristig eine ausreichende Nährstoffversorgung der Böden zu gewährleisten. Die im Untersuchungsgebiet gängige Praxis Ernterückstände abweiden zu lassen bzw. zu verbrennen sollte unterbleiben und diese als Mulch auf der Bodenoberfläche verbleiben. Eine ganzjährige Bodenbedeckung hätte den Vorteil, bei hohen Verdunstungsraten potenziellen Feuchtigkeitsverlusten der Böden zu begegnen bzw. umgekehrt bei hohen Niederschlägen möglichen Verschlämmungen entgegenzuwirken. Durch die niedrigeren Bodentemperaturen würden die Mineralisationsraten gemindert sowie sukzessive Pflanzennährstoffe aus der Streu freigesetzt. Denkbar wäre in diesem Zusammenhang die Einführung reduzierter Bodenbearbeitung (reduced tillage) oder von Direktsaat-systemen (notillage), wie diese in Südbrasilien unter vergleichbaren Bedingungen schon seit längerem erfolgreich betrieben werden (BAYER et al. 2001, LANDERS 2001, MACHADO \& SILVA 2001). Die Gründe ihrer Einführung in den 1980er Jahren waren die gleichen, wie sie aktuell in Ostbolivien zu beobachten sind: zunehmende Bodendegradation und Ertragsrückgänge.

\subsubsection{Zusammenfassung}

Die höchsten Kohlenstoff- und Stickstoffverluste sind unter konventioneller Bearbeitung im Boden in den ersten 20 Jahren nach der Konversion in eine ackerbauliche Nutzung zu verzeichnen. Der Abbau verläuft exponentiell, d.h. die höchsten Raten treten innerhalb der ersten 5-10 Anbaujahre auf. Mittlere relative Verluste der Oberbodenvorräte liegen in diesem Zeitraum bei 5-8 \% jährlich. Tonarme Böden weisen in den ersten Anbaujahren deutlich höhere Verluste auf als tonreiche. Die relativen Abbauraten liegen um 12-16 \% über denen tonreicher Böden, in denen die organische Bodensubstanz länger vor Freisetzung und Mineralisation geschützt ist. Nach langjährigem kontinuierlichem Anbau sind die Unterschiede nur noch gering ausgeprägt. Grundsätzlich liegen die relativen Verluste tonarmer Böden über denen tonreicher Böden, jedoch können die absoluten Verluste auf diesen nach mehrjähriger Nutzung höher ausfallen. Unabhängig von der Bodenart reduziert sich das Ausgangsniveau von SOC und $\mathrm{N}_{\mathrm{t}}$ nach mehr als 20-jähriger Ackernutzung im Oberboden um etwa die Hälfte. Ungefähr zu diesem Zeitpunkt stellt sich zudem ein neues Gleichgewicht 
zwischen Anlieferung und Abbau der organischen Substanz im Boden ein. Die relativen Abbauraten von SOC und $\mathrm{N}_{t}$ befinden sich in einer ähnlichen Größenordnung, obgleich die Verluste beim organischen Kohlenstoff generell etwas höher ausfallen.

Im Untersuchungsgebiet betragen die absoluten Verluste in den ersten 10 Anbaujahren im Mittel 10-12 $\mathrm{t} \mathrm{ha}^{-1}$ für SOC bzw. 1,2-1,5 $\mathrm{t} \mathrm{ha}^{-1}$ für $\mathrm{N}_{\mathrm{t}}$. Nach langjähriger Nutzung liegt die mittlere Reduktion bei 17-21 t ha ${ }^{-1}$ (SOC) bzw. $2 \mathrm{t} \mathrm{ha}^{-1}\left(\mathrm{~N}_{\mathrm{t}}\right)$. Allgemein gelten bereits nach einer Nutzungsdauer von mehr als 10 Jahren alle Ackerböden als schwach humos, die tonarmen Böden (v.a. sandige Lehme) nach mehr als 20 Jahren überdies als humusarm. Die kontinuierliche Bewirtschaftung hat über den Verlust organischer Bodensubstanz somit zu deutlichen Nährstoffdefiziten geführt, die insbesondere auf den lange Jahre konventionell bearbeiteten sandigen Lehmen bereits zahlreiche Flächenumwandlungen in eine extensive Weidenutzung nach sich gezogen hat. Demgegenüber lassen die tonreicheren Lehme aufgrund ihrer höheren Ausgangsgehalte länger einen produktiven Anbau erwarten, wobei die derzeit gängigen Bewirtschaftungsweisen auch auf diesen Böden eine kontinuierliche Nährstoffverarmung nach sich ziehen, weshalb mittelfristig eine analoge Entwicklung wie auf den tonarmen Substraten wahrscheinlich ist.

\subsection{3 Änderung der Lagerungsdichte unter ackerbaulicher Nutzung}

Die Anfälligkeit von Böden gegenüber Verdichtungen ist im wesentlichen von den spezifischen Bodeneigenschaften, der Bodenfeuchte und der Bewirtschaftungsart abhängig (BARBER et al. 1989, IMHOFF et al. 2004, KOZLOwSKI 1999). Schäden durch Bodenverdichtungen können erhebliche Störungen der Bodenfunktionen hervorrufen (Kap.5.1).

Mit Zunahme der Lagerungsdichte reduziert sich das Porenvolumen, insbesondere im Grobporenbereich, wodurch Einschränkungen im Luft- und Wasserhaushalt eines Bodens auftreten (BRONICK \& LAL 2005, CENTURION et al. 2004, LAL 1996a). Verdichtungen sind sowohl natürlicher Natur (BARBER 1995, FABIOLA et al. 2003), treten aber vor allem als unmittelbare Folge mechanischer Bodenbelastungen auf. Die Wahrscheinlichkeit von Verdichtungen steigt mit zunehmender Betriebsgröße und dem Grad der Mechanisierung (Assouline 2002, ABu-Hamdeh 2003). Somit stellen diese speziell in der industriellen Landwirtschaft ein großes Problem dar (Beutler \& CENTURIOn 2004, BotTA et al. 2004), sind aber bei unsachgemäßer Bodenbearbeitung auch in weniger technisierten Systemen möglich (ANIKWE et al. 2003). Mechanisch induzierte Verdichtungen sind vornehmlich auf den Oberboden beschränkt, können aber infolge von Druckfortpflanzungen bis in Tiefen von über $1 \mathrm{~m}$ reichen (KOZLOWSKI 1999). Unterbodenverdichtung gelten als besonders problematisch, da diese oft irreversibel sind (BERLi 2001, KELLER 2004). Auch Viehhaltung verursacht in der oberflächennahen Bodenzone z.T. gravierende Verdichtungsschäden (AREVALO et al. 1998, BARBER 1995, IMHOFF et al. 2000). 
Verdichtungen haben Auswirkungen auf praktisch alle Bodenfunktionen, weshalb es sich um einen der wesentlichen Bodendegradation auslösenden Prozesse handelt (CANILLAS \& SALOKHE 2001, IMHOFF et al. 2004, KelLER 2004). Nicht zuletzt ziehen Verdichtungen erhebliche ökonomische Kosten nach sich, da der Bearbeitungsaufwand auf verdichteten Böden allgemein höher ist, ebenso wie potenzielle Ertragsrückgänge theoretisch einen höheren Einsatz an Düngemitteln erfordern würden (DAVIES 1996, LAL 1997). BEUTLER \& CENTURION (2004) ermittelten in einer Studie in Südbrasilien auf einem sandig-tonigen Lehm schon nach einmaliger mechanischer Bearbeitung ohne zusätzliche Düngergaben eine Reduktion der Sojabohnenerträge um 25 \%. In der gleichen Größenordnung liegen die Ergebnisse von ABU-HAMDEH (2003), der Ertragsminderungen von 15-27 \% (Mais) auf einem tonigen Lehm im Jordan allein auf zunehmende Bodenverdichtungen infolge mechanischer Beanspruchung zurückführt. Zwar schaffen Bearbeitungsprozesse eine gewisse Lockerung im Oberboden, dieser Effekt ist aber in der Regel zeitlich begrenzt, da die diese instabil ist und sich der Boden nach Bearbeitung durch Einwirkung von Schwerkraft und Niederschlägen schnell wieder setzt (LAMPURLANÉS \& CANTERO-MARTÍNEZ 2003).

\section{Bodenart}

Mit Verdichtungen gehen, bezogen auf die Ertragsfähigkeit eines Bodens, Verschlechterungen der Bodenstruktur (Gefüge) einher (BRIDGES \& VAN BAREN 1997). Zwar besitzen feinkörnige Böden aufgrund allgemein höherer Humusgehalte und einer daraus resultierenden besseren Aggregierung eine höhere mechanische Belastbarkeit als grobkörnige Böden (ZHANG et al. 1997), dennoch sind diese anfälliger gegenüber Verdichtungen (IMHOFF et al. 2004). Ausschlaggebend für diese scheinbar widersprüchliche Tatsache sind unterschiedliche Bodenfeuchtegehalte. Allgemein gilt, dass mit steigenden Bodenfeuchtegehalten die Anfälligkeit feinkörniger Böden gegenüber Druckbelastungen zunimmt (BARBER et al. 1989, BotTA et al. 2004). Demnach nimmt der Zeitraum in dem sich feinkörnige Böden gut bearbeiten lassen mit steigendem Feinsubstratanteil ab, während die Wahrscheinlichkeit potenzieller Verdichtungen steigt. Bei sandigen Böden ist der Effekt der optimalen Bearbeitungszeit aufgrund ihrer geringen Wasserspeicherkapazität kaum von Bedeutung (KoZLOWSKI 1999).

Sind bei feinkörnigen Böden vornehmlich die Bodenfeuchtegehalte und die Bewirtschaftungsweise für Verdichtungen ausschlaggebend, gelten aufgrund ihrer natürlichen Eigenschaften Böden mit einem heterogenem Korngrößenspektrum als verdichtungsempfindlich (CENTURION et al. 2004). Durch die Zerstörung von Aggregaten infolge mechanischer Belastungen werden feine Bodenpartikel freigesetzt, die durch Verlagerung mit dem Sickerwasser sukzessive den Porenraum zwischen gröberen Partikeln ausfüllen. Davon besonders betroffen sind sandige bzw. sandig-tonige Lehme, da diese wegen ihres zumeist niedrigen Gehaltes an organischer Substanz eine nur geringe Aggregatstabilität besitzen. Das gleiche 
Prinzip findet bei natürlichen Verdichtungsprozessen statt, bei denen Tonpartikel mit dem Sickerwasser in tiefere Bodenschichten perkolieren und sich dort im Porenraum absetzen (Einlagerungsverdichtung). Je nachdem wie locker ein Boden in seinem ursprünglichen Zustand gelagert ist (Vorverdichtung), können Verdichtungen unter gleichen Bodenarten bei gleicher Bewirtschaftungsweise unterschiedlich hoch ausfallen. So ermittelten ARAUJO et al (2004) nach langjähriger konventioneller Bearbeitung auf einem sandig-tonigen Lehm in Südbrasilien eine Zunahme der Lagerungsdichte im Oberboden $(0-20 \mathrm{~cm})$ um $26 \%$, während CENTURION et al. (2004) bei gleichen Rahmenbedingungen aber geringerer Vorverdichtung auf eine Steigerung von $51 \%$ kommt.

Im Untersuchungsgebiet unterliegt ein großer Teil der Böden einer Kombination aus natürlicher und mechanisch induzierter Verdichtung (BARBER 1995). Das gilt insbesondere für die humusarmen sandigen Lehme, die ihre Hauptverbreitung westlich des Río Grande haben. In den feinkörnigeren Böden der östlichen Alluvialebene sind Verdichtungen mehrheitlich Folge der Bodenbearbeitung bei zu hohen Feuchtegehalten. Einer Studie von BARBER et al. (1989) zufolge, steigt die Anfälligkeit der Alluvialböden gegenüber Verdichtungen mit zunehmenden Feinsubstratanteil, während lehmige Sande mit einem Ton- und Schluffgehalt von unter $18 \%$ am wenigsten gefährdet sind. Sind die Böden verdichtet, ist eine natürliche Regeneration des Bodengefüges nur bedingt möglich, da die dominierenden Tonminerale (Glimmer, Kaolinit) dieser Region überwiegend nicht quellfähig sind (BARBER 1995). Theoretisch ist die Wahrscheinlichkeit von Verdichtungen im nördlichen Untersuchungsraum bei zwei Anbauzyklen pro Jahr höher als im Süden. In den Gebieten der Agroindustrie steigt das Risiko durch den vergleichsweise hohen Maschineneinsatz und die beträchtlichen Farmgrößen zusätzlich (Abb.6.2).

\section{Zeitliche Änderung}

Verschiedene Studien belegen, dass bereits mit der Rodung gravierende Verdichtungen im Boden einhergehen können. Der allein auf unterschiedliche Rodungstechniken zurückgehende Anstieg der Lagerungsdichte im Oberboden ( 0-15 cm) kann zwischen 10-20\% betragen (BARBER \& ROMERO 1994, ENEJI et al. 2003, LAL 1996a). Die Größenordnungen decken sich mit denen im Review von KozLOwsKi (1999) zitierten Werten. Die mittleren Zunahmen bei mechanisch ausgeführter Rodung liegen generell über denen des traditionellen slash and burn Systems, allerdings können auch bei letztgenanntem z.T. Verdichtungen im Boden auftreten (ENEJI et al. 2003, LAL 1996a).Im Untersuchungsgebiet zeigt sich, dass bei mechanisierter Bodenbearbeitung bereits innerhalb weniger Jahre markante Verdichtungsschäden im Boden auftreten können (Tab.35). In den ersten Anbaujahren sind auf sandigen Lehmen 20\%ige Zunahmen der Lagerungsdichten in den Oberböden möglich. In den Folgejahren verändern sich die Werte kaum, können aber im Extremfall 50 \% über dem Ausgangsniveau liegen (BARBER 1995). 
Tab. 35: Veränderung von Lagerungsdichte und Porenvolumen unter kontinuierlichem Ackerbau im Untersuchungsraum

\begin{tabular}{|c|c|c|c|c|c|c|c|c|c|}
\hline \multirow{2}{*}{$\begin{array}{c}\text { Region } \\
\text { Produzent }\end{array}$} & \multirow[b]{2}{*}{ Jahre } & \multirow[b]{2}{*}{ Bodenart } & \multirow[b]{2}{*}{$\begin{array}{l}\text { Tiefe } \\
{[\mathrm{cm}]}\end{array}$} & \multicolumn{3}{|c|}{ Lagerungsdichte $\left(d_{b}\right)$} & \multicolumn{3}{|c|}{ Porenvolumen (PV) } \\
\hline & & & & $\begin{array}{c}\text { Wald } \\
{\left[\mathrm{g} \mathrm{cm}^{-3}\right]}\end{array}$ & $\begin{array}{c}\text { Nutzung } \\
{\left[\mathrm{g} \mathrm{cm}^{-3}\right]}\end{array}$ & $\begin{array}{c}\text { Zunahme } \\
{[\%]}\end{array}$ & $\begin{array}{c}\text { Wald } \\
{[\text { Vol.-\%] }}\end{array}$ & $\begin{array}{l}\text { Nutzung } \\
\text { [Vol.-\%] }\end{array}$ & $\begin{array}{c}\text { Abnahme } \\
{[\%]}\end{array}$ \\
\hline${ }^{1}$ Las Brechas & \multirow[t]{3}{*}{1,5} & \multirow[t]{3}{*}{ SL } & $0-5$ & 0,94 & 1,15 & 22 & 65 & 57 & 12 \\
\hline \multirow[t]{2}{*}{ Mennoniten } & & & $15-20$ & 1,26 & 1,67 & 33 & 52 & 37 & 29 \\
\hline & & & $25-30$ & 1,38 & 1,71 & 24 & 48 & 35 & 26 \\
\hline${ }^{1}$ Las Brechas & \multirow[t]{3}{*}{14} & \multirow[t]{3}{*}{$\mathrm{SCL}$} & $0-5$ & 1,00 & 1,51 & 51 & 62 & 43 & 31 \\
\hline \multirow{2}{*}{ Mennoniten } & & & $15-20$ & 1,24 & 1,70 & 37 & 53 & 36 & 33 \\
\hline & & & $25-30$ & 1,38 & 1,81 & 31 & 48 & 32 & 34 \\
\hline${ }^{1}$ Las Brechas & \multirow[t]{3}{*}{18} & \multirow[t]{3}{*}{ SL } & $0-5$ & 1,01 & 1,36 & 35 & 62 & 49 & 21 \\
\hline \multirow[t]{2}{*}{ Mennoniten } & & & $15-20$ & 1,27 & 1,49 & 17 & 52 & 44 & 16 \\
\hline & & & $25-30$ & 1,33 & 1,64 & 23 & 50 & 38 & 23 \\
\hline${ }^{1}$ Saavedra & \multirow[t]{6}{*}{2} & \multirow[t]{6}{*}{ SL } & $0-5$ & 1,12 & 1,43 & 28 & 58 & 46 & 20 \\
\hline \multirow[t]{5}{*}{ Agroindustrie } & & & $5-10$ & 1,27 & 1,49 & 17 & 52 & 44 & 16 \\
\hline & & & $10-15$ & 1,36 & 1,51 & 11 & 49 & 43 & 12 \\
\hline & & & $15-20$ & 1,41 & 1,54 & 9 & 47 & 42 & 10 \\
\hline & & & $20-25$ & 1,44 & 1,58 & 10 & 46 & 40 & 12 \\
\hline & & & $30-35$ & 1,48 & 1,55 & 5 & 44 & 42 & 6 \\
\hline${ }^{1}$ Saavedra & \multirow[t]{6}{*}{6} & \multirow[t]{6}{*}{ SL } & $0-5$ & 1,12 & 1,41 & 26 & 58 & 47 & 19 \\
\hline \multirow[t]{5}{*}{ Agroindustrie } & & & $5-10$ & 1,27 & 1,49 & 17 & 52 & 44 & 16 \\
\hline & & & $10-15$ & 1,36 & 1,62 & 19 & 49 & 39 & 20 \\
\hline & & & $15-20$ & 1,41 & 1,62 & 15 & 47 & 39 & 17 \\
\hline & & & $20-25$ & 1,44 & 1,58 & 10 & 46 & 40 & 12 \\
\hline & & & $30-35$ & 1,48 & 1,54 & 4 & 44 & 42 & 5 \\
\hline${ }^{2}$ Las Brechas & 1 & $\mathrm{CL}$ & $0-20$ & 1,23 & 1,49 & 21 & 54 & 44 & 18 \\
\hline \multirow[t]{7}{*}{ Mennoniten } & 5 & SL & $0-20$ & 1,23 & 1,31 & 7 & 54 & 51 & 6 \\
\hline & 15 & SL & $0-25$ & 1,23 & 1,42 & 15 & 54 & 46 & 13 \\
\hline & \multirow[t]{3}{*}{25} & \multirow[t]{3}{*}{ SL } & $0-10$ & 1,23 & 1,48 & 20 & 54 & 44 & 18 \\
\hline & & & $10-21$ & 1,23 & 1,69 & 37 & 54 & 36 & 32 \\
\hline & & & $21-40$ & 1,51 & 1,78 & 18 & 43 & 33 & 24 \\
\hline & 29 & SL & $0-10$ & 1,23 & 1,43 & 16 & 54 & 46 & 14 \\
\hline & & & $10-45$ & 1,51 & 1,69 & 12 & 43 & 36 & 16 \\
\hline${ }^{3}$ Las Brechas & 1 & $\mathrm{~L}$ & $0-15$ & 1,06 & 1,15 & 8 & 60 & 57 & 6 \\
\hline Kleinbauern & & & $15-40$ & 1,37 & 1,37 & 0 & 48 & 48 & 0 \\
\hline & 3 & SL & $0-10$ & 1,06 & 1,24 & 17 & 60 & 53 & 11 \\
\hline & & & $10-40$ & 1,37 & 1,37 & 0 & 48 & 48 & 0 \\
\hline & 5 & SL & $0-20$ & 1,06 & 1,14 & 8 & 60 & 57 & 5 \\
\hline & 7 & $\mathrm{~L}$ & $0-10$ & 1,06 & 1,11 & 5 & 60 & 58 & 3 \\
\hline & & & $10-30$ & 1,37 & 1,41 & 3 & 48 & 47 & 3 \\
\hline${ }^{4}$ Lomerio & 7 & SL & $0-12$ & 1,13 & 1,29 & 14 & 57 & 51 & 11 \\
\hline Kleinbauern & & & $12-50$ & 1,39 & 1,46 & 5 & 48 & 45 & 6 \\
\hline & 24 & LS & $0-50$ & 1,13 & 1,30 & 15 & 57 & 51 & 11 \\
\hline
\end{tabular}

${ }^{1}$ Barber (1995); ${ }^{2}$ Markussen (2000); ${ }^{3}$ Karsten (2000); ${ }^{4}$ BlaschKe (1998)

Porenvolumen $(\mathrm{PV})$ errechnet aus Lagerungsdichte $\left(\mathrm{d}_{\mathrm{b}}\right)$ und Dichte der Festsubstanz $\left(\mathrm{d}_{\mathrm{f}}\right)$ nach: $P V=1-\left(d_{b} / d_{f}\right)$, wobei $\mathrm{d}_{\mathrm{f}}=2,65$ (USDA 1999)

Bodenarten: SL: sandy loam, SCL: sandy clay loam, L: loam, CL: clay loam (SoIL SURVEY STAFF 2003)

Deutliche Reduktionen des Porenvolumens sind speziell im Bereich der Pflugsohlen ausgeprägt. Mit Werten von unter $40 \%$ liegen diese bereits in einem für optimales Pflanzenwachstum kritischen Bereich (KUNTZE et al. 1988). Zu entsprechenden Ergebnissen kommen Untersuchungen aus Brasilien auf sandigen bzw. sandig-tonigen Lehmen unter mechanischer 
Bewirtschaftung. Sowohl die initiale Zunahme (MAIA \& RIBEIRO 2004) der Lagerungsdichten als auch das Niveau nach 20-30 Jahren kontinuierlicher Nutzung (Zunahme 16-40 \%, 0-20 $\mathrm{cm}$ ) bewegen sich in der gleichen Größenordnung wie die in Ostbolivien ermittelten Werte (ARAujo et al. 2004, CENTURION et al. 2004).

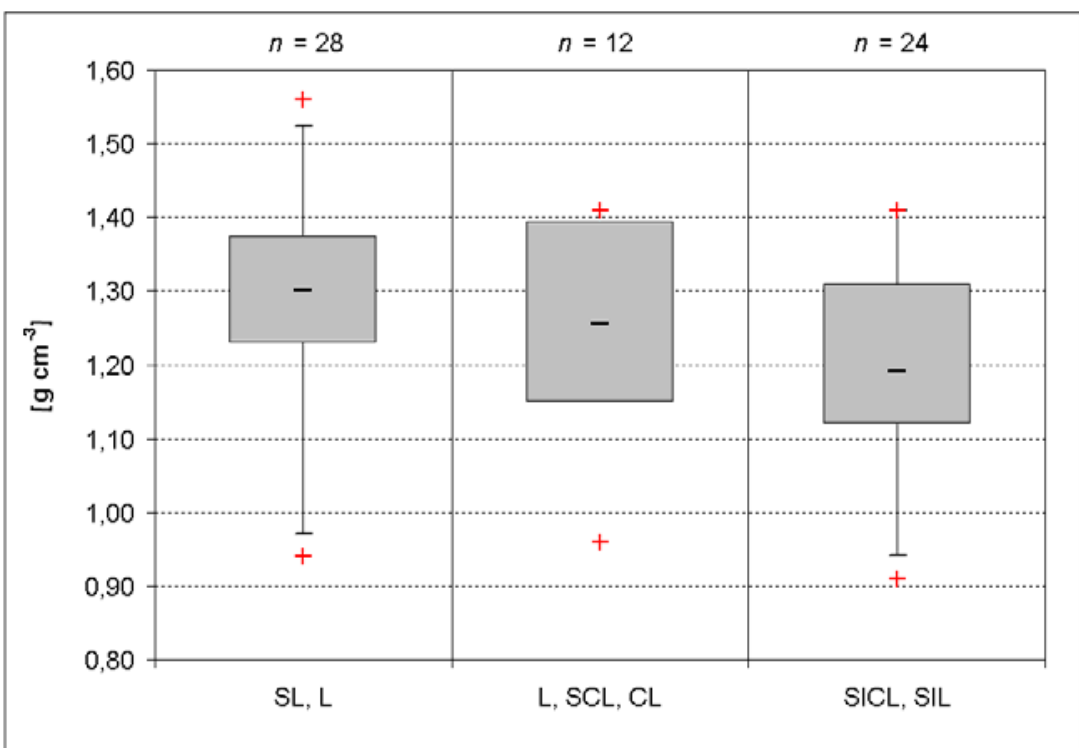

(SL: sandy loam, L: loam, SCL: sandy clay loam, CL: clay loam, SICL: silty clay loam SIL: silty loam)

Abb: 25: Boxplots der initialen Lagerungsdichten in Abhängigkeit von der Bodenart (Oberboden)

Weniger gravierend sind potenzielle Verdichtungen unter traditioneller Bewirtschaftung. KARSTEN (2000) und BLASCHKE (1998) ermittelten im Untersuchungsraum nach 7-jähriger Nutzung mittlere Zunahmen der Lagerungsdichte im Oberboden von 5-14 \%. Damit liegen die Werte in der Größenordnung, die DECHERT et al. (2004) in slash and burn Systemen Indonesiens ermittelt haben (12\%, 0-10 cm). Auch MCGRATH et al. (2001) beziffern den mittleren Anstieg der Lagerungsdichte in kleinbäuerlichen slash and burn Systemen des brasilianischen Amazonasraums auf $13 \%$ (0-20 cm). ZHAO et al. (2005) ermittelten in China unter traditioneller, nicht mechanischer Bearbeitung der Felder eine Zunahme der Lagerungsdichte auf einem lehmigen Sand von nur $11 \%$ nach 50 Jahren.

\subsubsection{Ableitung initialer Lagerungsdichten im Untersuchungsgebiet}

Da eine Regionalisierung der Lagerungsdichten auf Grundlage der vorhandenen Daten nicht möglich war (Kap.5.1.1), wurden aus den Bodendaten (Tab.6) unter Ergänzung einer Studie von BARBER (1995), mittlere initiale Lagerungsdichten der Oberböden abgeleitet. Die Zusammenfassung der Bodenarten und die Interpretation der Lagerungsdichten in Bezug zum Wurzelwachstum orientiert sich an den Ausführungen des Natural Resources Conservation Service (USDA 1999) (Tab.36). 
Tab. 36: Allgemeine Beziehung zwischen Lagerungsdichte und Wurzelwachstum auf Grundlage der Bodenart

\begin{tabular}{lccc}
\hline soil texture & $\begin{array}{c}\text { ideal bulk densities } \\
{\left[\mathbf{g ~ c m}^{-3}\right]}\end{array}$ & $\begin{array}{c}\text { bulk densities that may } \\
\text { affect root growth }\left[\mathbf{g ~ c m}^{-3}\right]\end{array}$ & $\begin{array}{c}\text { bulk densities that restrict } \\
\text { root growth }\left[\mathbf{g ~ c m}^{-3}\right]\end{array}$ \\
\hline sands, loamy sands & $<1.60$ & 1.69 & $>1.80$ \\
sandy loams, loams & $<1.40$ & 1.63 & $>1.80$ \\
sandy clay loams, loams, clay & $<1.40$ & 1.60 & $>1.75$ \\
loams & $<1.30$ & 1.60 & $>1.75$ \\
silts, silt loams & $<1.40$ & 1.55 & $>1.65$ \\
silt loams, silty clay loams & $<1.10$ & 1.49 & $>1.58$ \\
sandy clays, silty clays, some & $<1.10$ & 1.39 & $>1.47$ \\
clay loams (35-45 \% clay) & & & Quelle: USDA 1999
\end{tabular}

Die höchsten natürlichen Lagerungsdichten finden sich in den Oberböden sandiger Lehme und liegen im Mittel bei 1,28 $\pm 0,14 \mathrm{~g} \mathrm{~cm}^{-3}$. Dabei deuten die hohen Maximalwerte an, dass diese Böden aufgrund ihrer geringeren Gehalte an organischer Substanz stärker natürlichen Verdichtungsprozessen unterliegen (Abb.25). Mit steigendem Feinsubstratanteil im Boden sinken die Lagerungsdichten, während gleichzeitig die Porenvolumina zunehmen (Tab.37). Sandig-tonige bzw. tonige Lehme besitzen natürliche Lagerungsdichten von durchschnittlich $1,24 \pm 0,15 \mathrm{~g} \mathrm{~cm}^{-3}$ und schluffige bzw. schluffig-tonige Lehme von 1,20 $\pm 0,13 \mathrm{~g} \mathrm{~cm}^{-3}$. Nach SCHLICHTING et al. (1995) weisen die Oberböden damit im unbearbeiteten Zustand geringe bis mittlere Lagerungsdichten auf. Mittlere Porenvolumina liegen je nach Feinsubstratanteil zwischen 52-55 \pm 5 Vol.-\%, demnach besteht ein ausgewogenes Verhältnis zwischen Porenraum und Bodenpartikeln (Mineralien, organische Substanz).

\subsubsection{Bodenverdichtung im Untersuchungsgebiet - regionale Analyse}

Für die Darstellung des aktuellen Zustandes wurden Daten aus dem Untersuchungsgebiet analysiert und für die Hauptbodenarten mittlere Veränderungen von Lagerungsdichte und Porenvolumen unter mechanischer Bodenbearbeitung abgeleitet (Tab.37). Potenzielle Veränderungen im slash and burn System lassen sich aufgrund der mangelnden Datengrundlage nicht gesichert quantifizieren. Nach den zitierten Literaturangaben kann in diesem System allgemein von einer Zunahme der Lagerungsdichte im Oberboden in der Größenordung von $10 \%$ ausgegangen werden, womit in der Regel keine negativen Veränderungen der bodenphysikalischen Eigenschaften einhergehen. Untersuchungen von BLASCHKE (1998) und KARSTEN (2000) zufolge konnten unter manueller Bewirtschaftung im Untersuchungsgebiet keine Abnahmen der nutzbaren Feldkapazitäten festgestellt werden. Die Lagerungsdichten befinden sich zu jedem Zeitpunkt in einem für das Wurzelwachstum optimalen Bereich (Tab.35). Anders zeigt sich die Situation unter mechanisierter Bodenbearbeitung. Bereits nach wenigen Jahren konventioneller Nutzung konstatiert BARBER (1995) signifikante Reduktionen 
des Porenvolumens im Oberboden von 20-30 \%. Davon betroffen ist vornehmlich der Grobsowie z.T. der Mittelporenbereich, womit deutliche Reduktionen der Infiltrationsraten und der nutzbaren Feldkapazitäten einhergehen. Zu entsprechenden Ergebnissen kommt MARKUSSEN (2000), der nach langjähriger mechanischer Bearbeitung drastische Abnahmen des pflanzenverfügbaren Bodenwassers in der Größenordnung von 50-80 \% festgestellt hat. Die Lagerungsdichten unter mechanischer Bearbeitung zeigen speziell im Bereich zwischen $15-30 \mathrm{~cm}$ Bodentiefe Werte, die das Wurzelwachstum bereits erheblich beeinflussen können (Tab.35). Für sandige Lehme liegt dieser Wert nach Angaben des NRCS bei 1,63 $\mathrm{g} \mathrm{cm}^{-3}$. In Anlehnung an DADDOW \& WARRINGTON (1983) muss auf sandigen Lehmen in Abhängigkeit vom Sandgehalt etwa zwischen 1,60-1,75 $\mathrm{g} \mathrm{cm}^{-3}$ mit spürbaren Einschränkungen im Wurzelwachstum gerechnet werden (Abb.26).

Tab. 37: Ausgangsniveau und mittlere Veränderung von Lagerungsdichte und Porenvolumen durch mechanische Bodenbearbeitung

\begin{tabular}{|c|c|c|c|c|c|c|c|c|c|}
\hline \multicolumn{2}{|c|}{ Nutzungszeitraum } & \multicolumn{2}{|l|}{ inital } & \multicolumn{3}{|c|}{$<10$ a } & \multicolumn{3}{|c|}{$>10$ a } \\
\hline Bodenart & $\begin{array}{c}\mathbf{d}_{\mathbf{b}} \\
{\left[\mathrm{g} \mathrm{cm}^{-3}\right]}\end{array}$ & $\begin{array}{c}\text { PV } \\
{[\text { Vol.-\%] }}\end{array}$ & $\begin{array}{l}\text { Tiefe } \\
{[\mathrm{cm}]}\end{array}$ & $\begin{array}{c}\mathbf{d}_{\mathbf{b}} \\
{\left[\mathrm{g} \mathrm{cm}^{-3}\right]}\end{array}$ & $\begin{array}{c}\text { PV } \\
\text { Vol.-\% }\end{array}$ & $\begin{array}{l}\text { Tiefe } \\
{[\mathrm{cm}]}\end{array}$ & $\begin{array}{c}\mathbf{d}_{\mathbf{b}} \\
{\left[\mathrm{g} \mathrm{cm}^{-3}\right]}\end{array}$ & $\begin{array}{c}\text { PV } \\
{[\text { Vol.-\%] }}\end{array}$ & $\begin{array}{l}\text { Tiefe } \\
{[\mathrm{cm}]}\end{array}$ \\
\hline \multicolumn{10}{|c|}{ sandige Lehme (SL), Lehme (L) } \\
\hline MW & 1,28 & 52 & 21 & 1,50 & 43 & 21 & 1,59 & 40 & 23 \\
\hline Stabw & 0,14 & 5 & 11 & 0,14 & 5 & 11 & 0,13 & 5 & 11 \\
\hline Max & 1,56 & 65 & 40 & 1,71 & 57 & 45 & 1,84 & 49 & 45 \\
\hline Min & 0,94 & 41 & 5 & 1,15 & 35 & 5 & 1,35 & 31 & 5 \\
\hline$n$ & 28 & 28 & 28 & 19 & 19 & 19 & 29 & 29 & 29 \\
\hline \multicolumn{10}{|c|}{ sandig-tonige Lehme (SCL), tonige Lehme (CL), Lehme (L) } \\
\hline MW & 1,24 & 53 & 28 & 1,53 & 42 & 22 & 1,66 & 37 & 28 \\
\hline Stabw & 0,15 & 6 & 14 & 0,06 & 2 & 13 & 0,12 & 5 & 12 \\
\hline Max & 1,41 & 64 & 43 & 1,62 & 47 & 44 & 1,81 & 44 & 40 \\
\hline Min & 0,96 & 47 & 5 & 1,41 & 39 & 5 & 1,48 & 32 & 5 \\
\hline$n$ & 12 & 12 & 12 & 15 & 15 & 15 & 8 & 8 & 8 \\
\hline \multicolumn{10}{|c|}{ schluffige Lehme (SIL), schluffig-tonige Lehme (SICL) } \\
\hline MW & 1,20 & 55 & 21 & 1,32 & 50 & 19 & 1,47 & 45 & 21 \\
\hline Stabw & 0,13 & 5 & 9 & 0,17 & 6 & 8 & 0,09 & 3 & 11 \\
\hline Max & 1,41 & 66 & 38 & 1,52 & 60 & 30 & 1,59 & 49 & 45 \\
\hline Min & 0,91 & 47 & 5 & 1,07 & 43 & 5 & 1,34 & 40 & 11 \\
\hline$n$ & 24 & 24 & 24 & 10 & 10 & 10 & 11 & 11 & 11 \\
\hline
\end{tabular}

Auf Grundlage der regionalisierten Bodenarten und der aus der Satellitenbildanalyse bestimmten Nutzungsperioden und regionalen Anordnung der Landnutzungssysteme, wurden die aus den Studien abgeleiteten Veränderungen im GIS analysiert und potenzielle Schwankungsbreiten der Lagerungsdichten regional abgeleitet (Abb.27). 


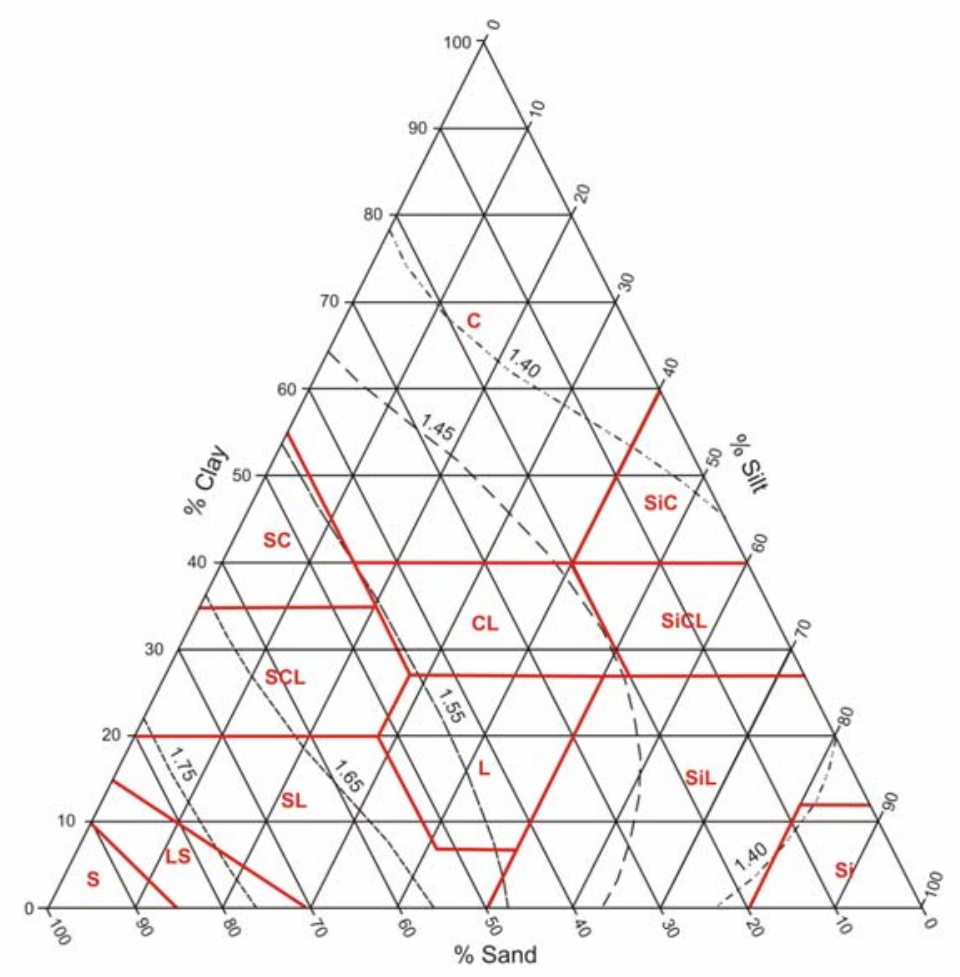

Abb. 26: Lagerungsdichten, ab denen Einschränkungen im Wurzelwachstum auftreten (nach DADDOW \& WARRINGTON 1983)

\section{Verdichtungsrate}

Bei Ableitung der Mittelwerte für das Untersuchungsgebiet ergab sich die zeitliche Einteilung von weniger und mehr als 10 Jahren kontinuierlichem Anbau aus der Quantität der verfügbaren Meßdaten. Die deutlichsten Veränderungen sind bei heterogener Korngrößenverteilung zu erkennen (Tab.37). Sandig-tonige (SCL) und tonige Lehme (CL) zeigen nach langjähriger mechanischer Bearbeitung eine durchschnittliche Zunahme der Lagerungsdichten von $34 \%$ auf 1,66 $\pm 0,12 \mathrm{~g} \mathrm{~cm}^{-3}$. Im Bereich der Pflugsohle erhöht sich der Wert auf 1,68 \pm $0,11 \mathrm{~g} \mathrm{~cm}^{-3}(n=7)$. Demnach befindet sich ein Großteil der Böden zu diesem Zeitpunkt in einem Verdichtungszustand, der ernste Konsequenzen für das Wurzelwachstum hat und durch die Reduktion des Porenvolumens auf unter 40 Vol.-\% erhebliche Einschnitte im Wasser- und Lufthaushalt mit sich bringt. In regionaler Hinsicht handelt es sich um den größten Teil der Ackerflächen in der zentralen Zone sowie um diverse Flächen in der Expansionszone, die schon seit längerem kontinuierlich bewirtschaftet werden (Abb.27).

Auf sandigen Lehmen (SL) ist nach langjähriger ackerbaulicher Nutzung eine mittlere Erhöhung der Lagerungsdichten um $24 \%$ auf 1,59 $\pm 0,13 \mathrm{~g} \mathrm{~cm}^{-3}$ (Pflugsohlenbereich: 1,62 \pm $0,12 \mathrm{~g} \mathrm{~cm}^{-3}, n=23$ ) festzustellen. Demnach äußern sich maschinell induzierte Verdichtungen auf diesen Böden weniger gravierend, gleichwohl auch auf sandigen Lehmen markante kultivierungsbedingte Verdichtungen auftreten können, die partiell über $1,70 \mathrm{~g} \mathrm{~cm}^{-3}$ betragen und damit nach den Schwellenwerten vom NRCS in einem Bereich liegen, in deren Folge mit 
Beeinträchtigungen der Ertragsfähigkeit gerechnet werden muss. Die betreffenden Bereiche befinden sich ausschließlich in der zentralen Zone (Abb.27).

Die Lagerungsdichten der schluffigen (SIL) und schluffig-tonigen Lehme (SICL) erhöhen sich im Oberboden nach langjähriger mechanischer Bewirtschaftung im Mittel um $22 \%$ auf 1,47 $\pm 0,09 \mathrm{~g} \mathrm{~cm}^{-3}$ (Pflugsohlenbereich: $1,51 \pm 0,09 \mathrm{~g} \mathrm{~cm}^{-3}, n=6$ ). Damit befinden sich die meisten Böden laut NRCS noch nicht in einem Zustand, der ernste Konsequenzen bezüglich der Durchwurzelung erwarten ließe. Wird dagegen die Einteilung von DADDOW \& WARRINGTON (1983) als Interpretationsgrundlage gewählt, muss auf diesen Böden ab Lagerungsdichten von 1,40-1,45 $\mathrm{g} \mathrm{cm}^{-3}$ mit Einschränkungen im Wurzelwachstum gerechnet werden. Demzufolge sind auch auf den feinkörnigen Böden des Untersuchungsgebietes vergleichsweise hohe Verdichtungsschäden wahrscheinlich. Dies umso mehr, da sich ein großer Teil dieser Böden in einer Zone mit zwei Anbauzyklen pro Jahr befindet. Über das Jahr gesehen bedeutet das allgemein eine hohe mechanische Beanspruchung sowie die die Gefahr, die Böden zum falschen Zeitpunkt, d.h. bei zu hohen Bodenfeuchtegehalten zu bearbeiten. Dieses Problem wir durch die beträchtlichen Schlaggrößen der agroindustriellen Anbauflächen zusätzlich verstärkt. Die entsprechenden Bereiche umfassen fast ausschließlich die am längsten bewirtschafteten Flächen in der Expansionszone (Abb.27).

Allgemein weniger kritisch stellt sich die Situation bei allen Bodenarten innerhalb der ersten 10 Anbaujahre dar. Zwar können in diesem Zeitraum partiell Verdichtungsschäden auftreten, dennoch befinden sich die Lagerungsdichten tendenziell nicht auf einem das Wurzelwachstum limitierenden Niveau (Tab.37). Indes ist davon auszugehen, dass bei Fortdauer der derzeit gängigen mechanischen Bewirtschaftungsweisen auch auf den weniger lang kultivierten Flächen in den nächsten Jahren mit zunehmenden Verdichtungsproblemen gerechnet werden muss. In regionaler Hinsicht handelt es sich mehrheitlich um Ackerflächen in der Expansionszone. Bei den Kleinbauern im slash and burn System würden sich bei einer hypothetischen Zunahme der Lagerungsdichten von $10 \%$ in Abhängigkeit von der Bodenart Werte in der Größenordnung von $1,41 \pm 0,14 \mathrm{~g} \mathrm{~cm}^{-3}$ (SL, L), 1,37 $\pm 0,15 \mathrm{~g} \mathrm{~cm}^{-3}$ (SCL, CL, L) sowie 1,33 $\pm 0,13 \mathrm{~g} \mathrm{~cm}^{-3}$ (SIL, SICL) ergeben. Demnach wären in diesem System keine negativen Auswirkungen auf das Wurzelwachstum zu erwarten. Allenfalls bei hoher natürlicher Vorverdichtung könnten auf schluffigen und tonig-schluffigen Lehmen, dem Schema von DADDOW \& WARRINGTON (1983) zufolge, Einschränkungen bei der Durchwurzelung auftreten, die aber nicht systembedingt sind.

\section{Verdichtungsempfindlichkeit}

Hinsichtlich der Disposition von Böden zu verdichten, ist der Wassergehalt im Boden von entscheidender Bedeutung. Je höher die aktuelle Durchfeuchtung desto verdichtungsanfälliger sind Böden. Da feintexturierte Böden aufgrund ihres höheren Anteils an Fein- und Mittelporen eine höhere Wasserspeicherkapazität haben, steigt die Verdichtungsempfindlichkeit mit 
zunehmendem Tongehalt. Die Bodenkundliche Kartieranleitung (AG BODEN 1994) sieht bereits Böden mit einem Tongehalt von 10-20\% im stark feuchten Zustand als äußerst verdichtungsempfindlich an, während eine Bearbeitung von Böden mit Tongehalten von über $25 \%$ in diesem Zustand unterbleiben sollte. Folglich limitieren höhere Tongehalte das Zeitfenster, in dem der Boden seinen optimalen Feuchtebereich für die Bearbeitung hat. $\mathrm{Zu}$ gleichen Ergebnissen kommen BARBER et al. (1989), die lehmige Sande als die Böden mit der geringsten Verdichtungsempfindlichkeit im Untersuchungsgebiet ausweisen. Dieser Tatsache folgend, können in Abhängigkeit von der Bodenart, dem Landnutzungssystem und den hygrischen Bedingungen die feinkörnigen Böden im nördlichen Untersuchungsgebiet als die am empfindlichsten gegenüber potenziellen Verdichtungen klassifiziert werden. Dies nicht zuletzt, da in dieser Region wegen der größeren Niederschlagssicherheit und dem höheren Feuchteangebot zwei Anbauzyklen pro Jahr durchgeführt werden. Mithin steigt infolge ständiger Bearbeitungsprozesse die mechanische Beanspruchung der Böden ebenso wie die Gefahr, die Felder zum falschen Zeitpunkt zu bearbeiten. Dies umso mehr, da die Bodenvorbereitung für die Einsaat der wichtigsten Anbaukulturen Soja (April, Mai) und Sonnenblume (Februar, März) in einen Zeitraum fällt, in dem sich die Böden theoretisch nahe der Feldkapazität befinden (Tab. A4, Anhang).

Bei den Bodenarten umfasst die Einordnung v.a. tonige und schluffig-tonige Lehme, da diese den größten Flächenanteil an den feinkörnigen Bodenarten einnehmen. Beim Betriebssystem sind aufgrund des hohen Mechanisierungsgrades, der Schlaggrößen und einer geringen Diversifikation der Anbaufrüchte insbesondere agroindustrielle Felder verdichtungsgefährdet, während das Risiko in den Gebieten der Kleinbauern aufgrund des geringeren Maschineneinsatzes, kleinerer Schlaggrößen und einer höheren Diversifikation der Anbaukulturen vermeintlich am geringsten ist. In hygrischer Hinsicht sind die Böden im südlichen Teil des Untersuchungsgebiets weniger anfällig gegenüber potenziellen Verdichtungen. Hier spielen vermehrt die geringen Humusgehalte der Böden bei der Zunahme der Lagerungsdichten eine Rolle.

Insgesamt liefert die Vorgehensweise eine Annäherung an den aktuellen Verdichtungsstatus. Detailliertere Aussagen können auf der gewählten räumlichen Skala aufgrund der Komplexität von Faktoren die bei der Ausbildung von Bodenverdichtungen eine Rolle spielen nicht getroffen werden. Allerdings wären, um eine bessere Absicherung der Aussagen treffen zu können, gerade auf den feinkörnigen Böden ergänzende Dichtemessungen erforderlich. Festzuhalten bleibt, dass nach langjähriger Bewirtschaftung bei allen Bodenarten Verdichtungsschäden auftreten können. Wesentlich in diesem Zusammenhang ist die Art und Häufigkeit der durchgeführten Bearbeitungstechniken, die Vorverdichtung eines Bodens, die Bodenfeuchte zum Zeitpunkt der Bearbeitung, der Gehalt an organischer Bodensubstanz sowie die Schlaggrößen. Nicht zuletzt spielt aufgrund unterschiedlicher Bearbeitungsintensitäten auch die Anbaukultur selbst, respektive deren Rotation mit anderen Kulturen, eine Rolle. 


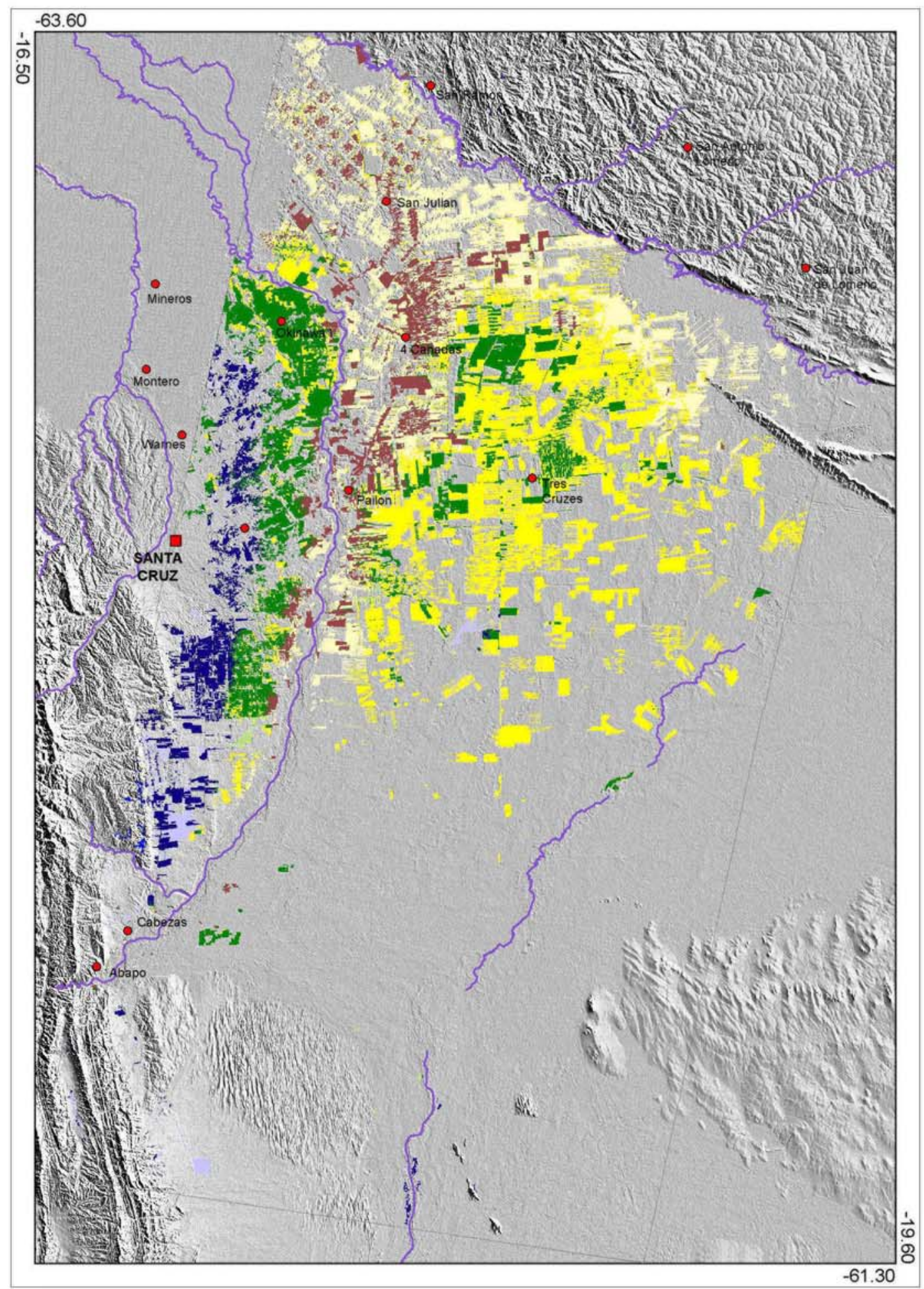

Bulk densities under agricultural use 2001 (topsoil) limits of variation $\left[\mathrm{g} \mathrm{cm}^{-3}\right]$

Soil textural classes

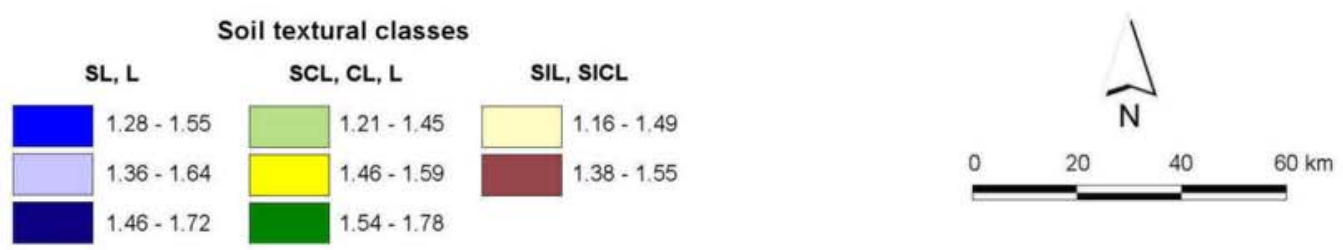

Abb. 27: Mittlere Lagerungsdichten im Oberboden von Ackerflächen 2001 


\subsubsection{Zusammenfassung}

Verdichtungen im Oberboden infolge mechanischer Einwirkungen treten bei allen Bodenarten auf. Die deutlichsten Veränderung sind auf sandig-tonigen und tonigen Lehmen zu verzeichnen. Im Mittel liegen die Lagerungsdichten nach langjähriger Bewirtschaftung bei 1,54-1,78 $\mathrm{g} \mathrm{cm}^{-3}$. Das bedeutet eine Steigerung um 24-43\% gegenüber dem Ausgangswert. Demgegenüber weisen sandige Lehme eine mittlere Spanne der Lagerungsdichten von 1,46$1,72 \mathrm{~g} \mathrm{~cm}^{-3}$ auf (Steigerung 14-34\%). In einer ähnlichen Größenordnung liegt die mittlere Zunahme auf schluffig-tonigen und schluffigen Lehmen mit 15-29\% auf 1,38-1,55 $\mathrm{g} \mathrm{cm}^{-3}$. Demnach ist auf allen langjährig mechanisch genutzten Ackerböden im Untersuchungsgebiet z.T. mit Verdichtungen im Oberboden zu rechnen, die das Wurzel- und somit auch das Pflanzenwachstum limitieren. Im kleinbäuerlichen slash and burn System sind allgemein keine negativen Veränderungen der bodenphysikalischen Eigenschaften zu verzeichnen.

Das Risiko schadhafter Bodenverdichtungen steigt unter mechanischer Bodenbearbeitung mit zunehmenden Feinsubstratanteil, da die Zeit, in der sich ein Boden in einem für die Bearbeitung optimalen Feuchtebereich befindet, kleiner wird. Im Untersuchungsgebiet bedeutet das eine hohe Gefährdung der feinkörnigen Böden im Norden und Nordwesten, da dort aufgrund der hygrischen Gegebenheiten zwei Anbauzyklen jährlich durchgeführt werden, ergo die Bearbeitungsintensität in dieser Region am höchsten ist. In Bezug auf das Betriebssystem steigt das Risiko schadhafter Bodenverdichtungen mit zunehmender Schlaggröße und geringer Diversifikation der Anbaukulturen. Demnach besteht das höchste Risiko in den Gebieten der agroindustriellen Anbauflächen, während dies in den Gebieten der Kleinbauern mit mechanisierter Bewirtschaftung weniger hoch eingeschätzt wird. 


\subsubsection{Bodenerosionsgefährdung durch Wind}

Bodenerosion ist ein natürlicher Prozess, der durch menschliche Aktivitäten beschleunigt wird (REICH et al. 2001). Weltweit hat die Erosion durch Wasser den größten Anteil an der durch die Landwirtschaft induzierten Bodendegradation (56\%), gefolgt von Winderosion (28 $\%$ ). Bodeninterne Veränderungen chemischer (12\%) und physikalischer (4 \%) Eigenschaften sind im Vergleich dazu weniger häufig die Ursache von Bodendegradation (OLDEMAN et al. 1991). Bodenerosionsraten unter natürlicher Vegetation sind im allgemeinen gering, steigen aber unter landwirtschaftlicher Nutzung, speziell wenn diese in konventioneller Form betrieben wird, rapide an. Extreme Ausmaße können dort auftreten, wo Flächen ohne Vegetationsbedeckung sind (REICH et al. 2001).

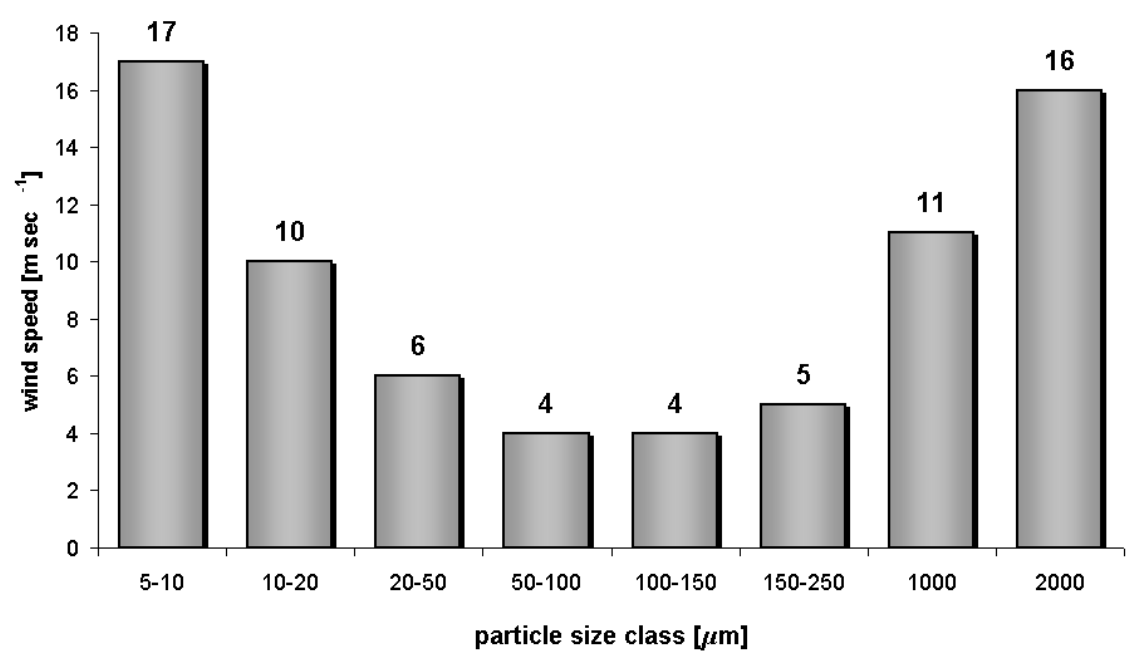
Abb. 28: Schwellenwerte der Windgeschwindigkeit zur Erosion von Bodenpartikeln in $15 \mathrm{~cm}$ Höhe
(nach CHEPIL \& WOODRUFF 1963)

Die von OLDEMAN et al. (1991) erarbeiteten Zahlen verdeutlichen, in welchen Umfang erosive Windereignisse zur Degradation der Böden beitragen können. Winderosion tritt vornehmlich auf bewirtschafteten Ackerflächen, aber auch als Folge von Überweidung auf (STERK 2003). Die Anfälligkeit eines Bodens gegenüber Winderosion ist im wesentlichen von der Bodenart, dem Gefüge und der Bodenfeuchte abhängig (BÖHNER et al 2003b, ZOBECK et al. 2003). Für die Mobilisierung von Bodenteilchen müssen in Abhängigkeit von der Partikelgröße bestimmte Schwellenwerte der Windgeschwindigkeiten überschritten werden (Abb.28). Die Ablösung mineralischer und organischer Partikel aus dem Bodenverband erfolgt sowohl durch die Einwirkung der aerodynamischen Kräfte des Windes (Kap. 5.1.4.2), als auch durch den Aufprall saltierender Bodenpartikel auf Kornoberflächen und Aggregate. Insbesondere durch Saltation gelangen feine Partikel in Suspension und können über weite Strecken transportiert werden. Da an diese der Hauptteil der Nährstoffe gebunden ist, reichern sich auf den Erosionsflächen grobe, nährstoffarme Sedimente an (PIMENTEL \& KOUNANG 1998). 
Nach Analysen von CHEPIL \& WodDRUFF (1963) wird deutlich, dass Bodenpartikel innerhalb der Grobschluff- bis Feinsandfraktion am leichtesten durch den Wind erodierbar sind (Abb. 28), da die Partikel in diesem Bereich meist im Einzelkorngefüge vorliegen. Mit abnehmender Korngröße nehmen die Kohäsionskräfte zwischen den Partikeln zu, wodurch die Aggregierung steigt und die Partikel erst durch die Zerstörung der Aggregate in den Transportprozess gelangen. Bei zunehmender Korngröße wiederum bestimmt das höhere Partikelgewicht deren geringere Erodierbarkeit (NEEMAN 1991). Nach CHEPIL (1941) gelten Bodenpartikel und Aggregate mit einer Größe von unter 0,84 $\mathrm{mm}$ als potenziell vom Wind erodierbar. NEEMAN (1991) ermittelte im Vergleich dazu einen Schwellen-wert von 0,63 mm. Gefährdet sind demnach v.a. sandige Böden, da in diesen die Anzahl und Stabilität der Bodenaggregate gering ist und Bodenpartikel leicht verlagert werden können (STOUT \& ZOBECK 1996). Dies tritt dann ein, wenn eine schützende Vegetationsdecke fehlt und sich der Boden im trockenem Zustand befindet. MERRILL et al. (1999) ermittelten, dass der Anteil der erodierbaren Fraktion im Boden in Trockenperioden um 27 \% höher liegt als in Feuchtperioden. Folglich ist Winderosion vornehmlich ein Phänomen das unter landwirtschaftlicher Nutzung in semi-ariden und ariden Räumen auftritt (LAL 2001, WHICKER et al. 2002, ZHAO et al 2005).
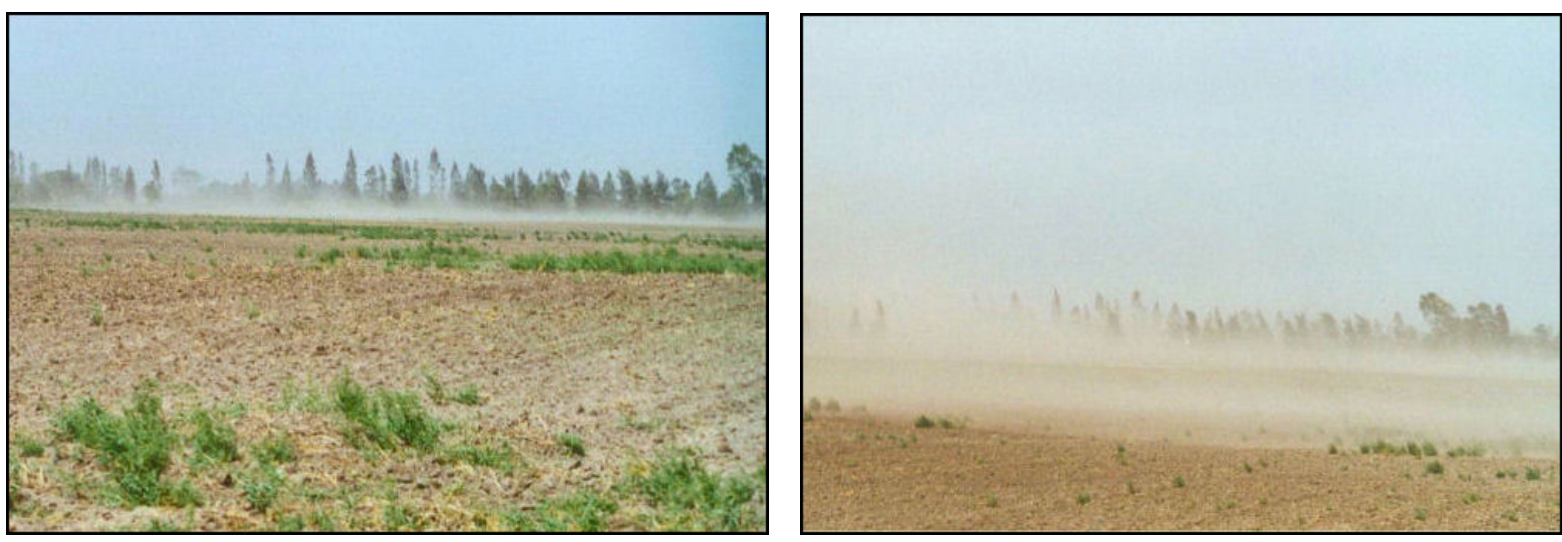

Abb. 29: Winderosionsereignisse im südlichen Untersuchungsgebiet (05.09.2002)

Potenzielle Schäden durch Winderosion können direkt auf den landwirtschaftlichen Nutzflächen (on-site Effekte), aber auch in weiterer Entfernung am Ort der Ablagerung (offsite Effekte) auftreten (GROß 2002). Während erstere im allgemeinen den Produktivitätsverlust der Böden umfassen, werden unter letzteren Schäden außerhalb der Erosionsflächen subsummiert (BLASCHKE et al. 2000, LAL 2001). Schäden durch Winderosion sind im globalen Vergleich in Südamerika eher wenig verbreitet. Der Anteil liegt bei ca. 8 \%. Die höchsten Winderosionsraten sind in Asien (41\%) und Afrika (34\%) zu verzeichnen (OLDEMAN et al. 1991). Winderosionsprozesse im südamerikanischen Raum sind vorwiegend aus den semi-ariden Bereichen Argentiniens bekannt (BUSCHIAZzo et al. 1999, PERI \& BloOMBERG 2002). 
Erosionsschutzmaßnahmen, wie bspw. die Anlage von Windschutzstreifen oder die Bedeckung des Bodens mit einer Mulchschicht, verringern das Risiko von Bodenabträgen erheblich (MichELs et al. 1998). BIELDERS et al. (2000) ermittelten im Vergleich mit unbedeckten Feldern auf gemulchten Böden eine Reduktion der Erosionsrate um 87 \%. Wie wichtig Erosionsschutz ist unterstreichen PIMENTEL \& KounANG (1998). Ihren Angaben zufolge kann allein während eines Niederschlags- oder Sturmereignisses ohne weiteres $1 \mathrm{~mm}$ Boden erodieren, was auf einen Hektar addiert 15 t ergibt. Die Wiederherstellung der Ausgangssituation würde unter natürlichen Umständen etwa 20 Jahre benötigen.

Die allgemeinen Ausführungen lassen erkennen, dass Winderosion vornehmlich auf den Agrarflächen im semi-ariden Teil des Untersuchungsgebietes ein Problem darstellt. Allerdings können in Trockenperioden auch die weiter nördlich gelegenen Gebiete von Winderosion betroffen sein. Studien über Ausmaß und Wirkungsweise von Winderosion wurden im Untersuchungsgebiet nicht durchgeführt. Einzig von DERPSCH (1974) existiert eine Studie für die zentrale Zone, in der die Winde hinsichtlich ihrer erosiven Wirkung ausgewertet wurden. Seinen Angaben zufolge liegt die Häufigkeit erosiver Winde mit Geschwindigkeiten von über $6 \mathrm{~m} \mathrm{~s}^{-1}$ bei etwa $12 \%$ pro Jahr; bereits ein Viertel erreicht mit ca. $5 \mathrm{~m} \mathrm{~s}^{-1}$ leicht erosive Wirkung (in 0,3 $\mathrm{m}$ Höhe). Die höchsten Windgeschwindigkeiten mit Maximalwerten von bis zu $25 \mathrm{~m} \mathrm{~s}^{-1}$ (in $14 \mathrm{~m}$ Höhe) treten in den Monaten Juni bis Oktober auf, einer Zeit in der die Böden hochgradig erosionsgefährdet sind, da sich auf den zu dieser Zeit oft unbedeckten Feldern die potenzielle Evapotranspiration erhöht und die Böden z.T. tiefgründig ausgetrocknet sind (Abb.29).

Nachfolgend werden die Windverhältnisse im Untersuchungsgebiet charakterisiert und anhand der natürlichen Bodeneigenschaften die Erodierbarkeit über die Ableitung eines KFaktors bestimmt. Dafür mussten die jeweiligen Unterfraktionen der Korngrößen rechnerisch abgeleitet werden, da diesbezügliche Messungen bei den im Untersuchungsgebiet durchgeführten Bodenstudien kaum vorgenommen wurden.

\subsubsection{Windverhältnisse im Untersuchungsgebiet}

Beobachtungen über die Windverhältnisse im Untersuchungsgebiet existieren nur wenige in geringer zeitlicher (Monatsbasis) und räumlicher Auflösung. Alle Angaben stammen vom staatlichen Wetterdienst SENAMHI, aufgezeichnet in 6 m Höhe.

Die im Untersuchungsgebiet vorherrschende Windrichtung ist $270^{\circ}-360^{\circ}$ (Abb.30), wobei der Anteil der Südwinde mit zunehmender Breitenlage ansteigt und ein mittleres Maximum in den Wintermonaten (Juni-September) aufweist. An den beiden nördlichen Stationen Concepción und San Javier dominieren im Jahresverlauf Winde aus N-NW (85\%) bei einer durchschnittlichen jährlichen Windgeschwindigkeit von ca. $4 \mathrm{~m} \mathrm{~s}^{-1}$. Der Anteil der Winde aus 
$\mathrm{S}-\mathrm{SE}$ ist im Jahresverlauf gleich. In den Wintermonaten liegen die Monatsmittelwerte mit $5 \mathrm{~m}$ $\mathrm{s}^{-1}$ leicht höher, mit möglichen Maxima von 7-8 $\mathrm{m} \mathrm{s}^{-1}$. Im Osten (San José, Chiquitos) liegt der Anteil der Winde aus nördlichen Richtungen im Jahresmittel bei 78 \% (davon $60 \%$ aus N). In den Wintermonaten kommen $27 \%$ der Winde aus Richtung S-SE. Die Windgeschwindigkeiten liegen im Jahresmittel bei $3,6 \mathrm{~m} \mathrm{~s}^{-1}$, mit nur geringen Unterschieden im Jahresverlauf. Die höchsten Windgeschwindigkeiten werden im September mit im Mittel 4,3 $\mathrm{m} \mathrm{s}^{-1}$, bei möglichen monatlichen Maxima von ca. $6 \mathrm{~m} \mathrm{~s}^{-1}$, erreicht. Im Westen (El Trompillo, Santa Cruz) liegt der Anteil der Nordwinde im Jahresverlauf bei $79 \%$ (davon $66 \%$ aus NW). Der Anteil der S-SE Winde liegt auch dort in den Wintermonaten bei etwa einem Viertel. Mit einem Jahresmittel von $5,7 \mathrm{~m} \mathrm{~s}^{-1}$ treten in dieser Region die höchsten Windgeschwindigkeiten auf. In den Wintermonaten liegt das Monatsmittel bei $6,4 \mathrm{~m} \mathrm{~s}^{-1}$, mit möglichen Maxima von bis zu $9 \mathrm{~m} \mathrm{~s}^{-1}$ (Abb.30, Tab.38).
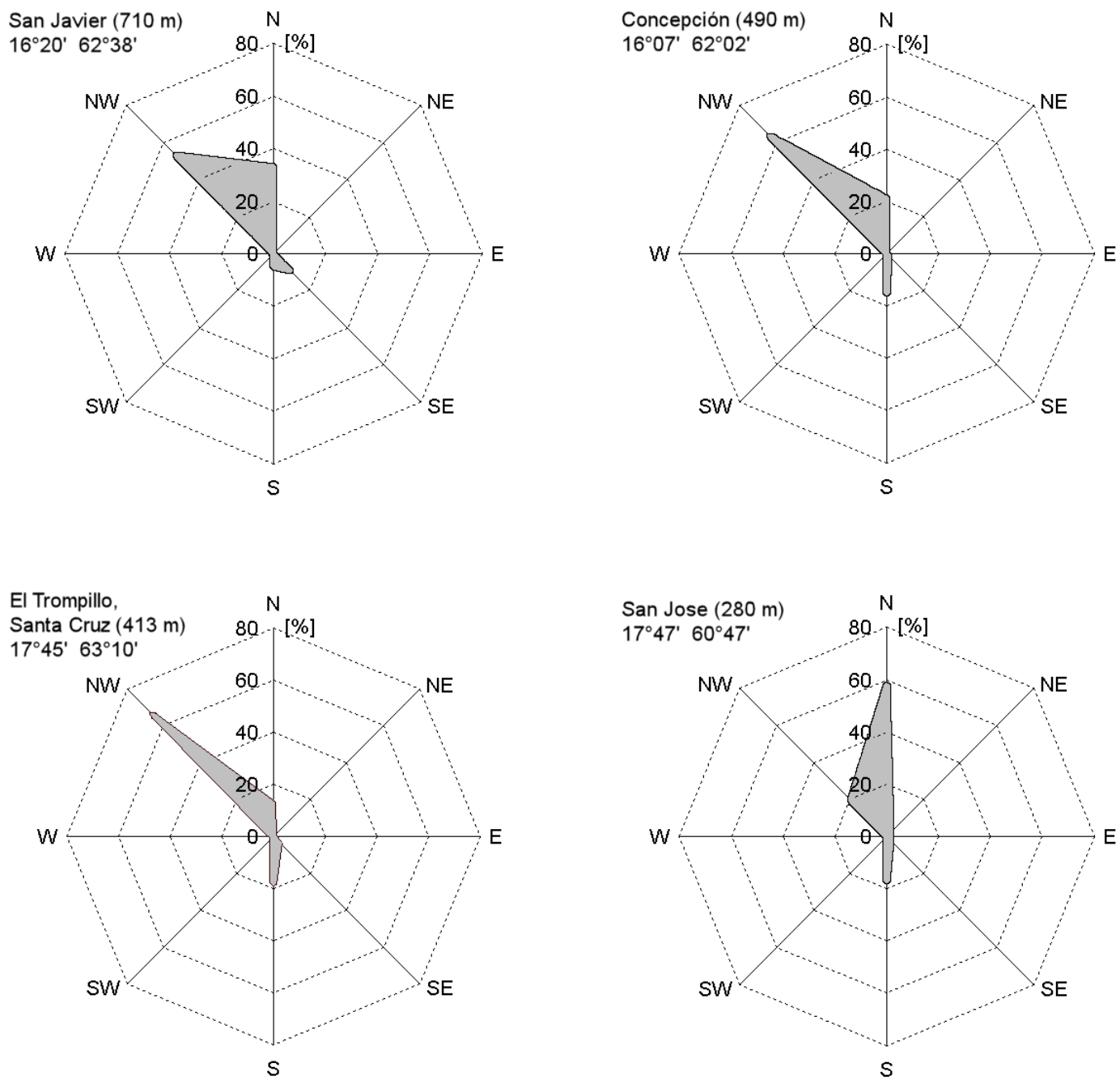

Abb. 30: Vorherrschende Windrichtungen im Untersuchungsgebiet 


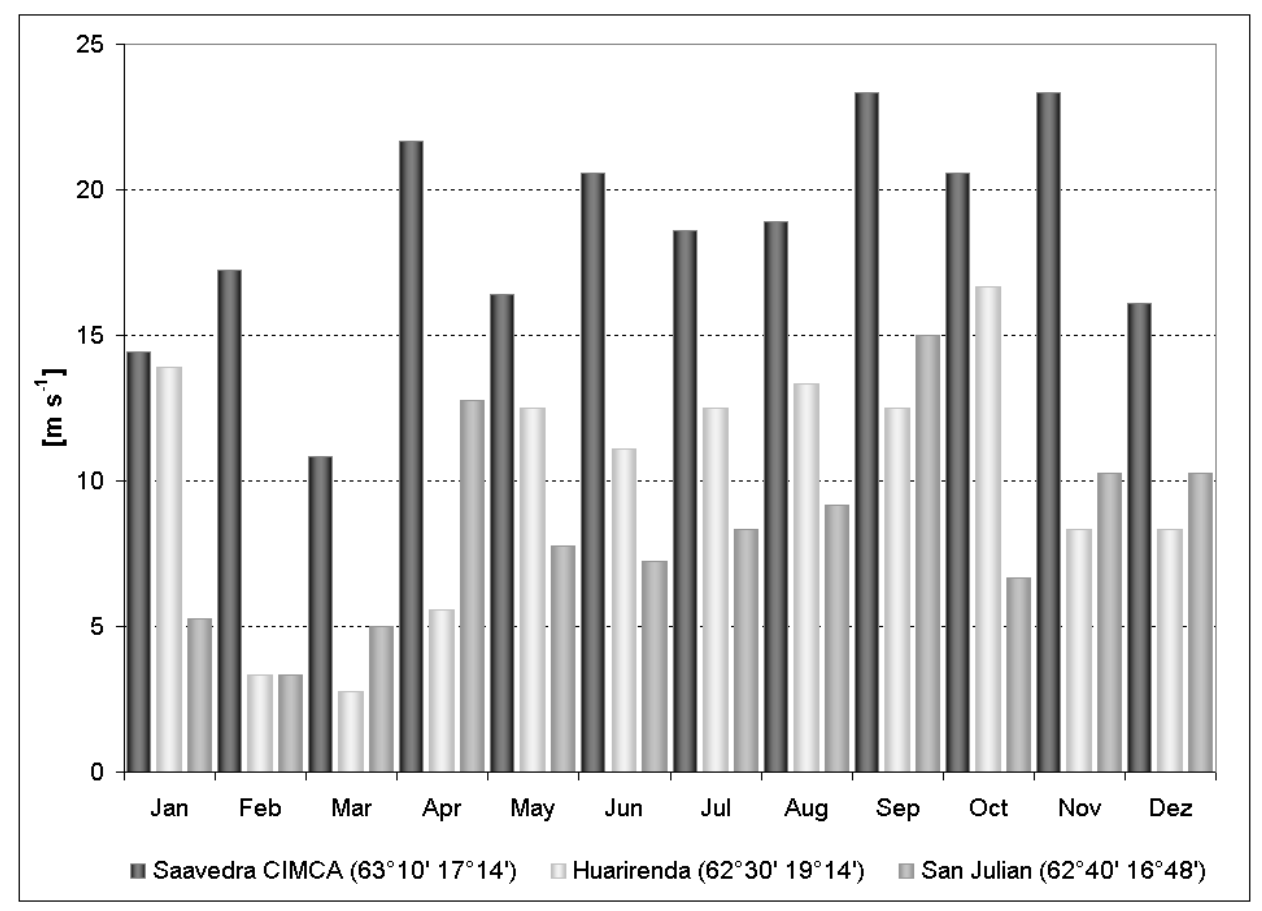

Abb. 31: Monatliche Tagesmaxima der Windgeschwindigkeit 1980

(Quelle: SENAMHI)

Tab. 38: Deskriptive Statistik der Windgeschwindigkeiten (Monatsmittel in $\mathrm{m} \mathrm{s}^{-1}$ )

\begin{tabular}{lccccccccccccc}
\hline & Jan & Feb & Mar & Apr & May & Jun & Jul & Aug & Sep & Oct & Nov & Dec & year \\
\hline San Javier (1960-1979) & & & & & & & & & & & \\
MW & 4,2 & 3,6 & 3,1 & 3,0 & 3,7 & 4,0 & 4,6 & 4,8 & 5,0 & 4,5 & 4,4 & 4,0 & 4,1 \\
Min & 2,6 & 1,5 & 1,0 & 1,5 & 1,5 & 1,5 & 2,1 & 2,1 & 2,6 & 2,6 & 2,6 & 1,0 & 2,3 \\
Max & 6,2 & 6,2 & 4,1 & 5,1 & 5,1 & 5,7 & 7,2 & 7,2 & 7,2 & 6,7 & 6,7 & 6,7 & 5,0 \\
Stabw & 1,0 & 1,4 & 0,9 & 0,9 & 1,0 & 1,3 & 1,3 & 1,5 & 1,2 & 1,1 & 1,1 & 1,5 & 0,7 \\
$n$ & 20 & 20 & 20 & 19 & 20 & 20 & 20 & 20 & 20 & 20 & 20 & 20 & 20
\end{tabular}

Concepcion (1960-1979)

$\begin{array}{llllllllllllll}\text { MW } & 4,3 & 3,7 & 3,4 & 3,3 & 3,4 & 4,3 & 5,0 & 5,5 & 5,3 & 5,0 & 4,7 & 4,3 & 4,4 \\ \text { Min } & 3,1 & 2,1 & 2,1 & 2,1 & 1,5 & 2,6 & 2,6 & 4,1 & 3,1 & 3,6 & 3,1 & 2,6 & 3,5 \\ \text { Max } & 5,7 & 5,1 & 4,6 & 4,6 & 5,1 & 6,2 & 7,2 & 7,7 & 6,7 & 6,7 & 7,2 & 6,2 & 5,1 \\ \text { Stabw } & 0,8 & 0,8 & 0,9 & 0,7 & 0,9 & 1,0 & 1,2 & 1,1 & 1,0 & 0,8 & 1,0 & 1,1 & 0,5 \\ n & 20 & 20 & 20 & 20 & 20 & 20 & 20 & 20 & 20 & 20 & 20 & 20 & 20\end{array}$

EI Trompillo, Santa Cruz (1960-1979, 1994-2003)

$\begin{array}{llllllllllllll}\text { MW } & 5,4 & 5,1 & 5,0 & 4,8 & 5,1 & 6,2 & 6,5 & 6,3 & 6,4 & 6,0 & 5,7 & 5,6 & 5,7 \\ \text { Min } & 1,5 & 3,1 & 3,1 & 3,1 & 2,6 & 4,1 & 4,1 & 4,6 & 5,1 & 4,1 & 4,1 & 3,6 & 4,3 \\ \text { Max } & 8,2 & 6,7 & 6,7 & 5,7 & 6,7 & 8,2 & 8,7 & 8,2 & 8,7 & 7,7 & 7,7 & 9,8 & 6,6 \\ \text { Stabw } & 1,3 & 0,8 & 1,0 & 0,8 & 0,9 & 1,0 & 1,1 & 0,9 & 0,9 & 1,0 & 0,9 & 1,4 & 0,5 \\ n & 30 & 30 & 30 & 30 & 30 & 30 & 30 & 30 & 30 & 30 & 30 & 30 & 30\end{array}$

San Jose de Chiquitos (1960-1979)

\begin{tabular}{llllllllllllll} 
MW & 3,6 & 2,8 & 2,8 & 3,3 & 3,4 & 3,4 & 3,6 & 3,8 & 4,3 & 4,0 & 4,1 & 3,6 & 3,6 \\
Min & 1,0 & 1,0 & 1,0 & 1,0 & 2,1 & 1,5 & 1,5 & 2,1 & 1,5 & 0,5 & 1,5 & 1,0 & 1,8 \\
Max & 7,2 & 4,6 & 4,1 & 4,6 & 4,6 & 4,6 & 5,1 & 6,2 & 6,2 & 6,2 & 6,2 & 6,2 & 4,5 \\
Stabw & 1,5 & 1,0 & 0,8 & 0,9 & 0,7 & 0,9 & 0,9 & 1,2 & 1,4 & 1,5 & 1,4 & 1,5 & 0,8 \\
$n$ & 20 & 19 & 19 & 19 & 19 & 19 & 20 & 20 & 19 & 19 & 19 & 18 & 19 \\
\hline
\end{tabular}


Die höheren Windgeschwindigkeiten in der zentralen Zone deuten zudem die monatlichen Tagesmaxima aus dem Jahr 1980 an (Abb.31). Demnach erreichen die Windgeschwindigkeiten in dieser Region (Saavedra) wiederholt Sturmstärke ${ }^{27}\left(>20 \mathrm{~m} \mathrm{~s}^{-1}\right)$, oder liegen im Bereich starker bis stürmischer Winde $\left(>15 \mathrm{~m} \mathrm{~s}^{-1}\right)$. Auch in der Chacoregion (Huarirenda) treten häufiger noch starke Winde mit Geschwindigkeiten von mehr als $10 \mathrm{~m} \mathrm{~s}^{-1}$ auf, wogegen die Windverhältnisse im nördlichen Untersuchungsraum (San Julian) ausgeglichener sind.

\subsubsection{Erosivität der Winde}

Die höhere Erodierbarkeit unbedeckter Bodenoberflächen wird durch Abbildung 32 verdeutlicht. Danach wird die in einer Referenzhöhe $\left(Z_{r}\right)$ gemessene Windgeschwindigkeit $\left(\mathrm{U}_{Z}\right)$ in Abhängigkeit von der Bodenrauigkeit unterschiedlich stark, bis auf null unmittelbar über der Bodenoberfläche, abgebremst. Die Höhe, in der $U_{Z}=0$ ist, wird über die sogenannte aerodynamische Rauigkeitslänge $\left(\mathrm{Z}_{0}\right)$ ausgedrückt. Die über der Bodenoberfläche auftretenden Windgeschwindigkeiten sind infolge der geringeren Reibungskräfte auf unbedeckten Böden $\left(Z_{0} \sim 1 \mathrm{~cm}\right)$ bis zum Erreichen von $Z_{\mathrm{r}}$ deutlich höher als bspw. bei geschlossenen Getreidebeständen $\left(Z_{0} \sim 10 \mathrm{~cm}\right)$. Demzufolge erhöht sich das Erosionsrisiko mit abnehmender Bodenrauigkeit.

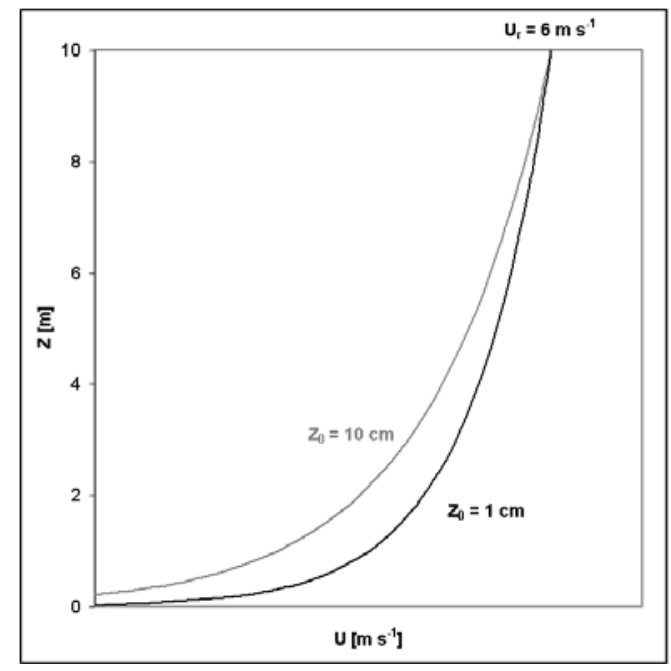

Abb. 32: Windprofile bei unterschiedlicher Bodenrauhigkeit

Unabhängig von der Bodenbedeckung steigt die Bodenrauigkeit mit steigendem Anteil stabiler Aggregate, weshalb Sandböden gegenüber Winderosion stärker anfällig sind, als Tonund Schluffböden (NEEMANN 1991). Innerhalb der durch Reibung beeinflussten bodennahen Grenzschicht (Prandtl-Schicht) treten Scherkräfte auf, die einen Impulsfluss aus der Luftströmung auf die Bodenoberfläche bewirken (Schubspannung). Diese Kraft ist für den

\footnotetext{
${ }^{27}$ nach Beaufort Skala
} 
Transport von Bodenpartikeln ausschlaggebend (BAGNOLD 1941). Die Stärke der Impulsübertragung wird über die Schubspannungsgeschwindigkeit ( $\left.U_{*}\right)$ ausgedrückt, die definiert ist durch:

$$
\mathrm{U}_{*}=\sqrt{\frac{\tau}{\rho}}
$$

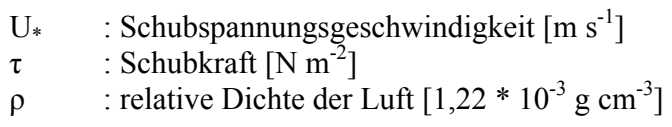

Unter Annahme einer neutralen Atmosphärenschichtung mit laminarem Strömungszustand kann die Zunahme der Windgeschwindigkeit mit der Höhe nach dem logarithmischen Windgesetz innerhalb der Prandtl- Schicht wie folgt beschrieben werden:

$$
\mathrm{U}_{(\mathrm{Z})}=\frac{\mathrm{U}_{*}}{\kappa} \ln \left(\frac{\mathrm{Z}}{\mathrm{Z}_{0}}\right) \quad \text { mit } \mathrm{Z}>\mathrm{Z}_{0}
$$

\footnotetext{
$\mathrm{U}_{(\mathrm{Z})} \quad$ : Windgeschwindigkeit in Höhe $\mathrm{Z}\left[\mathrm{m} \mathrm{s}^{-1}\right]$

$\mathrm{U}_{*} \quad$ : Schubspannungsgeschwindigkeit $\left[\mathrm{m} \mathrm{s}^{-1}\right]$

$\kappa \quad:$ Karman-Konstante $(\sim 0,4)$

$\mathrm{Z}_{0} \quad$ : aerodynamische Rauigkeitslänge
}

Die an den meteorologischen Stationen in einer bestimmten Referenzhöhe $\left(Z_{r}\right)$ aufgezeichneten Windgeschwindigkeiten $\left(\mathrm{U}_{\mathrm{r}}\right)$ lassen sich bei Kenntnis der aerodynamischen Rauigkeitslänge $\left(\mathrm{Z}_{0}\right)$ auf andere Höhen umrechnen. In diesem Fall gilt:

$$
\mathrm{U}_{\mathrm{r}}=\frac{\mathrm{U}_{*}}{\kappa} \ln \left(\frac{\mathrm{Z}_{\mathrm{r}}}{\mathrm{Z}_{0}}\right) \Rightarrow \mathrm{U}_{*}=\mathrm{U}_{\mathrm{r}} \frac{\kappa}{\ln \left(\mathrm{Z}_{\mathrm{r}} / \mathrm{Z}_{0}\right)} \Rightarrow \mathrm{U}_{(\mathrm{z})}=\mathrm{U}_{\mathrm{r}} \frac{\ln \left(\mathrm{Z} / \mathrm{Z}_{0}\right)}{\ln \left(\mathrm{Z}_{\mathrm{r}} / \mathrm{Z}_{0}\right)}
$$

Die im Untersuchungsgebiet in $Z_{\mathrm{r}}=6 \mathrm{~m}$ Höhe aufgezeichneten Windgeschwindigkeiten wurden unter Annahme einer hypothetischen Bodenrauigkeit für unbedeckte Böden von $\mathrm{Z}_{0}=$ $1 \mathrm{~cm}$ (OKE 1987) auf eine Höhe von $Z=0,15$ m umgerechnet. Die Höhe entspricht der, in der Chepil \& WoOdRufF (1963) Schwellenwerte der Windgeschwindigkeit für die Erosion von Bodenpartikeln bestimmt haben. Danach würde auf unbedeckten Böden etwa ab Windgeschwindigkeiten von $>9 \mathrm{~m} \mathrm{~s}^{-1}\left(Z_{\mathrm{r}}=6 \mathrm{~m}\right)$ Erosion einsetzten. Dieser Wert ist gut vergleichbar mit der von SAXTON et al. (2001) ermittelten Größenordnung von 5-7 m s${ }^{-1}\left(Z_{r}=\right.$ $3 \mathrm{~m})$. 
Die Darstellung der Windgeschwindigkeiten auf Monatsbasis kann lediglich einen Überblick über die vorherrschenden Windverhältnisse geben, ist aber als Grundlage für eine Häufigkeitsabschätzung in Bezug auf die Erosivität der Winde nicht ausreichend, da potenziell höhere Windgeschwindigkeiten über die Monatsmittelung nicht dargestellt werden. Eine gängige Annäherung zeitlicher Häufigkeiten von Windgeschwindigkeitsklassen lässt sich unter Einbezug einer mittleren Windgeschwindigkeit (z.B. Jahresdurchschnittsgeschwindigkeit) über die RAYLEIGH-Funktion [8] erzielen (Abb.33).

$$
\mathrm{h}(\mathrm{U})=\frac{\pi}{2} \frac{\mathrm{U}}{\overline{\mathrm{U}}^{2}} \exp ^{-\frac{\pi}{4}\left(\frac{\mathrm{U}}{\mathrm{U}}\right)^{2}}
$$

$\mathrm{h}(\mathrm{U}) \quad$ : Häufigkeit der Windgeschwindigkeit [\%]

$\mathrm{U} \quad$ : Windgeschwindigkeit (der Häufigkeitsklasse) $\left[\mathrm{m} \mathrm{s}^{-1}\right]$

$\overline{\mathrm{U}} \quad$ : mittlere Windgeschwindigkeit $\left[\mathrm{m} \mathrm{s}^{-1}\right]$
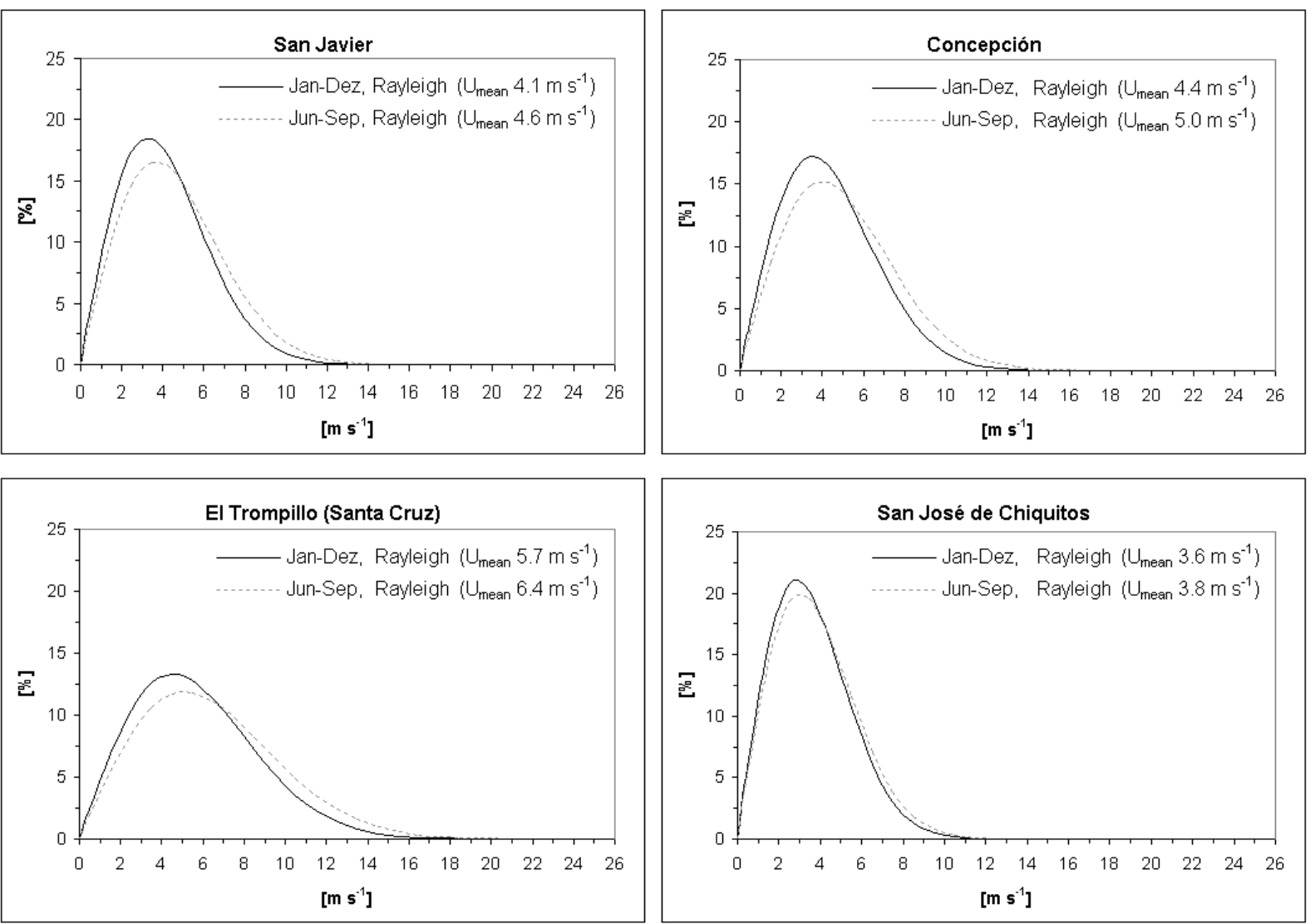

Abb. 33: Berechnete Häufigkeitsverteilung der Windgeschwindigkeit in \% nach Rayleigh

Die Häufigkeit erosiver Winde mit einer Geschwindigkeit von über $9 \mathrm{~m} \mathrm{~s}^{-1}$ (in $6 \mathrm{~m}$ Höhe) beträgt in der zentralen Zone (E1 Trompillo, Santa Cruz) im Jahresverlauf $14 \%$; werden nur die Wintermonate betrachtet, erhöht sich der Wert auf $21 \%$. In der nördlichen Untersuchungsregion (Concepción, San Javier) liegen die Häufigkeiten mit Werten von 4 \% (8 \%) bzw. $2 \%$ (5\%) deutlich niedriger. Am seltensten treten erosive Winde im Osten auf (San 
José de Chiquitos). Lediglich $1 \%$ der Winde wehen im Jahresverlauf mit Geschwindigkeiten von mehr als $9 \mathrm{~m} \mathrm{~s}^{-1}$. In der Trockenzeit liegt der Wert nur geringfügig höher (Abb.33).

\subsubsection{Erodibilität des Bodens}

Die relative Erodibilität des Bodens gegenüber Winderosion wird nachfolgend über die Ableitung eines Bodenerodierbarkeitsfaktors (K-Faktors) nach NEEMANN (1991) bestimmt. Da die Funktion in Bezug auf die Bodenart neben dem Ton- und Schluffgehalt auch den Anteil an Fein- und Feinstsand integriert, mussten aus der Korngrößenverteilung die jeweiligen Unterfraktionen rechnerisch ermittelt werden. Detaillierte Laboranalysen aller Korngrößenfraktionen des Feinbodens wurden bei den Studien des Untersuchungsgebiets, mit wenigen Ausnahmen, nicht vorgenommen.

\section{Ableitung der Korngrößenfraktionen}

Mit dem Problem, die vollständige Korngrößenverteilung über Interpolationsansätze durch einen Kurvenfit an gegebene Datenpunkte (z.B. Messdaten) zu approximieren, haben sich diverse Autoren beschäftigt (BITTELli et al 1999, DA Silva et al. 2004, PoSADAS et al. 2001, SKAGGS et al 2001). In diesem Zusammenhang häufig angewandte Verfahren sind Anpassungen von log-Normal Funktionen an die Korngrößensummenkurve (BUCHAN 1989). Diese allerdings setzen eine annähernde Gleichverteilung der Korngrößen im Boden voraus, wie es in der Natur nicht generell gegeben ist. NEMES et al. (1999) kommen in ihrer Betrachtung zu dem Schluss, dass die Anpassung der log-Normal Funktion an Messdaten, die geringste Genauigkeit der vier von ihnen getesteten Funktionen lieferte. HwANG et al. (2002) ermittelten unter sieben verschiedenen Methoden, dass bei allen von ihnen untersuchten Bodenarten die beste Anpassungen über den von FREDLUND et al. (2000) entwickelten Algorithmus erzielt wird. Der auch in vorliegender Studie verwendete Algorithmus arbeitet nach dem Prinzip der kleinsten Quadrate [9]. Dabei wird über stückweise Interpolation der quadrierte vertikale Abstand zwischen Kurve und Datenpunkt minimiert.

Der Algorithmus wurde auf die Messdaten mit Angaben der Unterfraktionen eingestellt und die Anpassungen bei guter Übereinstimmung für Profile gleicher Bodenarten übernommen, bei denen nur Daten der Hauptfraktionen Ton, Schluff und Sand vorlagen. Im Mittel konnte die Funktion mit einer Bestimmtheit von $\mathrm{r}^{2}=0,91$ (Median 0,97, $n=54$ ) an die Messwerte angepasst werden. Die gleiche Anpassungsgüte ergab sich bei den sandigen Lehmen $(n=24)$, den Böden des Untersuchungsgebiets, die hauptsächlich von Winderosion betroffen sind. Die Qualität der Anpassung am Beispiel sandiger Lehme demonstriert Abbildung 34. Anhand der generierten Korngrößensummenkurve konnten die prozentualen Verteilungen der Korngrößenfraktionen für alle Profile abgetragen werden. 

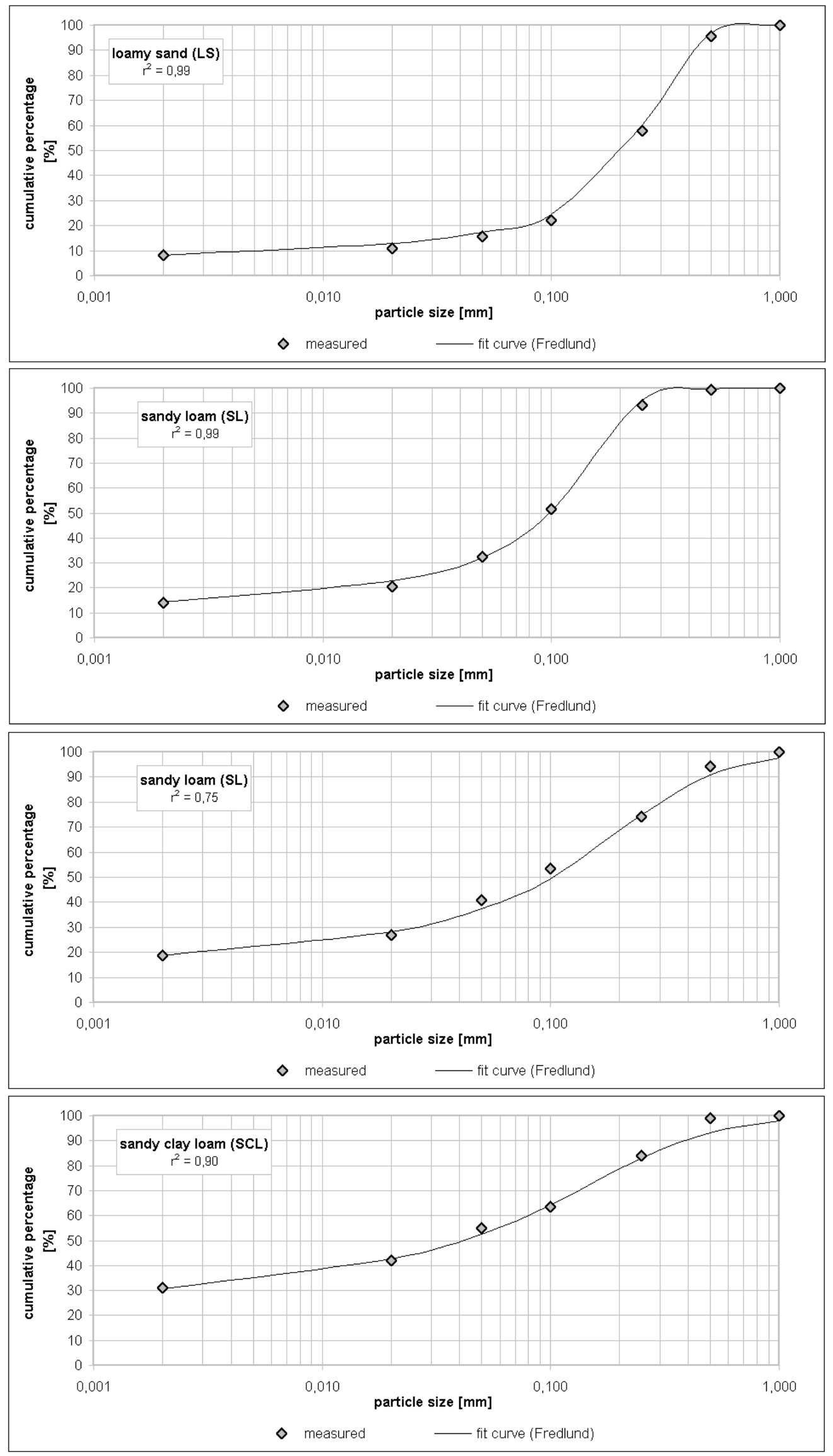

Abb. 34: Kurvenfit nach dem Algorithmus von FREDLuND et al. (2000) für sandige Lehme 


$$
\mathrm{P}_{\mathrm{p}}(\mathrm{d})=\frac{1}{\ln \left[\exp (1)+\left(\frac{\mathrm{a}_{\mathrm{gr}}}{\mathrm{d}}\right)^{\mathrm{n}_{\mathrm{gr}}}\right]^{\mathrm{m}_{\mathrm{gr}}}}\left[1-\left[\frac{\ln \left(1+\frac{\mathrm{h}_{\mathrm{rgr}}}{\mathrm{d}}\right)}{\ln \left(1+\frac{\mathrm{h}_{\mathrm{rgr}}}{\mathrm{d}_{\mathrm{m}}}\right)}\right]^{7}\right]
$$

$\mathrm{P}_{\mathrm{p}}(\mathrm{d})$ : Prozent (kumulativ) einer Korngrößenfraktion

$\mathrm{a}_{\mathrm{gr}} \quad$ : Fit-Parameter (bezieht sich auf den ersten Wendepunkt der Kurve)

$\mathrm{n}_{\mathrm{gr}} \quad$ : Fit-Parameter (bezieht sich auf maximale Steigung der Kurve)

$\mathrm{m}_{\mathrm{gr}} \quad$ : Fit-Parameter (bezieht sich auf die Krümmung der Kurve)

d : Partikeldurchmesser [mm]

$\mathrm{h}_{\mathrm{rgr}} \quad$ : residualer Partikeldurchmesser [mm]

$\mathrm{d}_{\mathrm{m}} \quad$ : kleinster Partikeldurchmesser [0,0001 mm]

\section{Bestimmung der K-Faktoren}

Der K-Faktor [dimensionslos], als Maß der Bodenerodierbarkeit sandiger Böden, wurde von NEEMANN (1991) in Windtunnelexperimenten empirisch ermittelt. Ausgedrückt wird die relative Empfindlichkeit eines Bodens gegenüber Winderosion, wenn dieser sich im trockenen, frisch bearbeiteten Zustand befindet und nicht durch Bodenbedeckung geschützt ist. Einfluss auf den K-Faktor haben die Textur, der SOM-Gehalt und die Aggregierung. Mittels einer Regressionsgleichung [10] werden die K-Faktoren als Funktion des gewogenen mittleren Durchmessers der Textur [12] und dem Anteil der Aggregate mit einem Durchmesser von $>0,63 \mathrm{~mm}$ berechnet. Letzterer lässt sich ebenfalls über eine Regressionsgleichung [11] auf Basis des SOM-Gehalts und dem Anteil der Ton-, Schluff- und Feinsandfraktionen ermitteln.

$$
\left.\log \mathrm{K}=1,24-4,21\left[\mathrm{GMD}_{\mathrm{t}}\right]-0,04 \text { [Aggregate }>0,63 \mathrm{~mm}\right]
$$

$\mathrm{GMD}_{\mathrm{t}}$

Aggregate $>0,63 \mathrm{~mm}$

: gewogener mittlerer Durchmesser der Textur [mm]

Korrekturfaktor

: Aggregate $>0,63 \mathrm{~mm}<4$ Gew.- $\%$, dann gilt $\mathrm{K} * 1,2\left(\mathrm{r}^{2}=0,92\right)$

$$
\mathrm{A}=-2,42+8,6\left[\log (\mathrm{SOM}-\text { Gehalt })+\left[\frac{\mathrm{T}+\mathrm{U}}{\mathrm{fS}+\mathrm{ffS}}\right]\right]
$$

A : Aggregatanteil $>0,63 \mathrm{~mm}$ [Gew.\%], nach $3 \mathrm{~mm}$ Vorsiebung $\left(\mathrm{r}^{2}=0,65\right)$

$$
\mathrm{GMD}_{\mathrm{t}}=\frac{\sum\left[\mathrm{d}_{\mathrm{i}} \mathrm{n}_{\mathrm{i}}\right]}{100}
$$

$\mathrm{d}_{\mathrm{i}} \quad$ : Klassenmitte der Aggregatfraktionen [mm]

$\mathrm{n} \quad$ : Anteil der Aggregatfraktion [Gew.-\%]

Je höher der berechnete K-Faktor, desto leichter sind Böden vom Wind erodierbar. Eine Einteilung wird nach den in Tabelle 39 aufgeführten Erodibilitätsklassen vorgenommen. Für 
die Bestimmung der Erosionsneigung der Böden des Untersuchungsgebiets wurden KFaktoren sowohl für die Profile unter ackerbaulicher Nutzung, als auch für die unter natürlicher Vegetation berechnet. Bei letztgenannten wurde eine hypothetische, kultivierungsbedingte Abnahme des SOM-Gehalts, wie unter Kapitel 5.1.2.1 beschrieben, angenommen und auf dieser Grundlage K-Faktoren abgeleitet.

Tab. 39: Erodibilitätsklassen (K-Faktor)

\begin{tabular}{ccc}
\hline Klasse & Erodibilität & K-Faktor \\
\hline K1 & sehr gering & $<0,1$ \\
K2 & gering & $0,1-0,3$ \\
K3 & mäßig & $0,3-0,5$ \\
K4 & stark & $0,5-1,0$ \\
K5 & sehr stark & $>1,0$ \\
\hline
\end{tabular}

Tab. 40: Korrelationskoeffizienten des K-Faktors mit SOM-Gehalt, Aggregierung und Textur

\begin{tabular}{|c|c|c|c|c|c|c|c|c|}
\hline \multicolumn{9}{|c|}{ Pearson Korrelation - Sandböden (LS, SL, SCL) } \\
\hline & SOM & $\mathrm{A}>0,63 \mathrm{~mm}$ & $\mathrm{gS}$ & $\mathrm{mS}$ & $\mathrm{fS}+\mathrm{ffS}$ & $\mathrm{gU}$ & $\mathrm{fU}$ & $\mathrm{T}$ \\
\hline \multicolumn{9}{|c|}{ gemessener SOM-Gehalt } \\
\hline $\mathrm{r}$ & $-0,29 * *$ & $-0,71 * * *$ & $-0,33 * *$ & $-0,32 * *$ & $0,75 * * *$ & n.s & $-0,30 * *$ & $-0,38 * * *$ \\
\hline$n$ & 67 & 67 & 67 & 67 & 67 & 67 & 67 & 67 \\
\hline \multicolumn{9}{|c|}{ hypothetischer SOM-Gehalt } \\
\hline $\mathrm{r}$ & $-0,26^{*}$ & $-0,65^{* * *}$ & $-0,47 * * *$ & $-0,61 * * *$ & $0,83 * * *$ & $0,42 * * *$ & n.s. & $-0,40 * *$ \\
\hline$n$ & 59 & 59 & 59 & 59 & 59 & 59 & 59 & 59 \\
\hline
\end{tabular}

$\alpha>0,05$ n.s., $\alpha<0,05 *, \alpha<0,01 * *, \alpha<0,001 * * *$

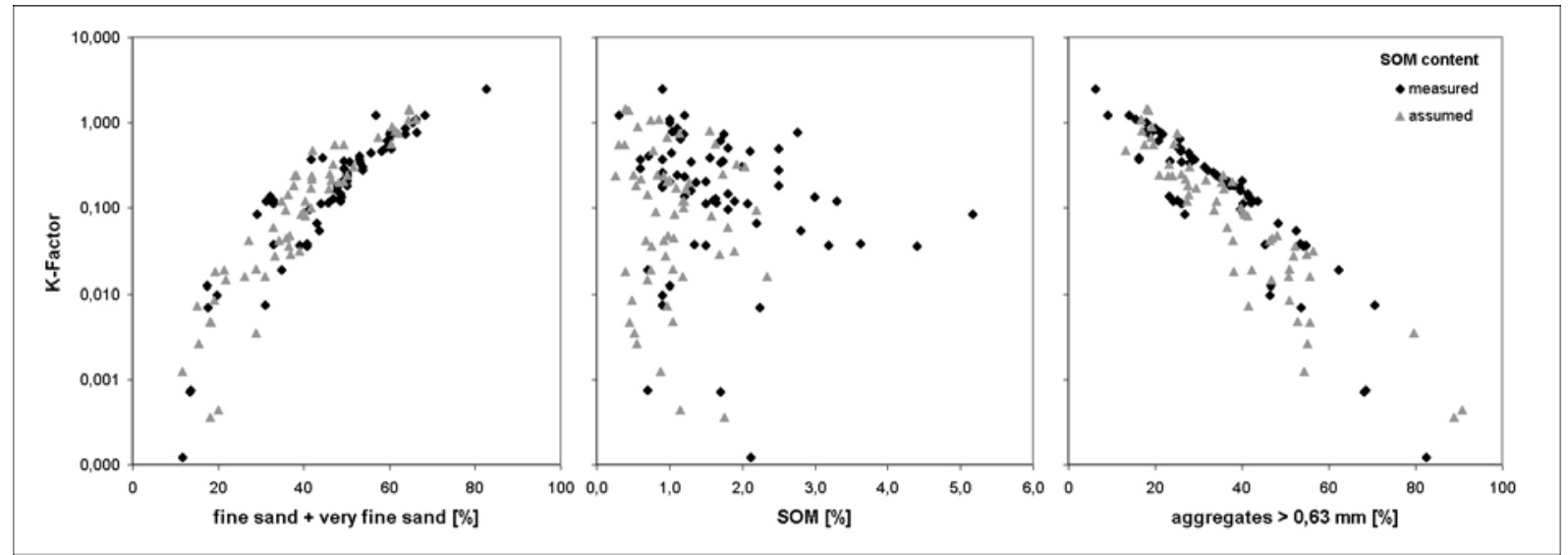

Abb. 35: Einfluss von Textur, SOM-Gehalt und Aggregierung sandiger Lehme auf die Erodibilität

Erwartungsgemäß wird die Erodibilität am stärksten von Textur und Aggregierung der Böden beeinflusst. Mit steigendem Anteil der Feinsandfraktionen nimmt die Erodibilität zu, während mit zunehmender Aggregierung bzw. zunehmendem Kornumfang die Tendenz 
gegenläufig ist, worauf signifikant negative Korrelationen mit dem K-Faktor hindeuten (Tab.40, Abb.35). Weniger eindeutig zeigt sich der Einfluss der organischen Substanz auf die Erodibilität. Zwar sind die Abhängigkeiten signifikant, allerdings auf geringerem Niveau. NeEMANN (1991) nimmt an, dass der direkte Einfluss der organischen Substanz auf die Erodibilität von anderen Bodeneigenschaften überlagert wird und diese eher sekundär über die Aggregatbildung im Boden wirkt.
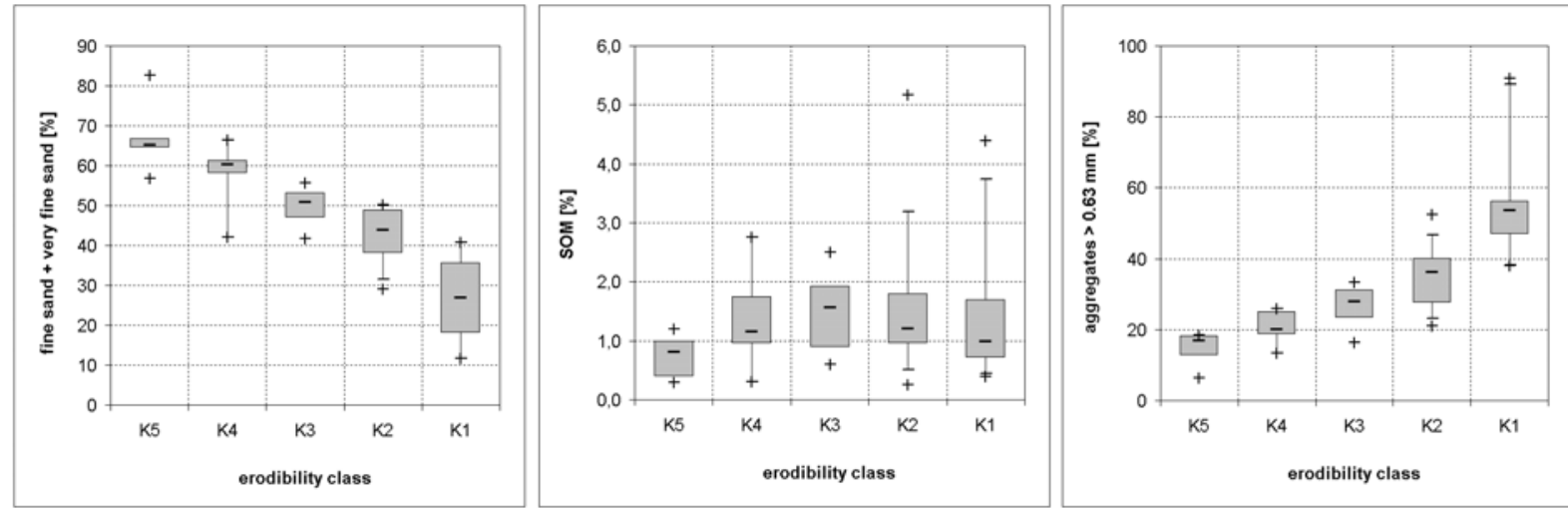

Abb. 36: Einfluss von Textur, SOM-Gehalt und Aggregierung sandiger Lehme auf die Erodibilität

Die höchste Erodibilität der Böden des Untersuchungsgebiets weisen sandige Lehme (SL) und lehmige Sande (LS) mit einem mittlerem Gewichtsanteil der Feinsandfraktionen von $66 \pm$ $6 \%$, der Aggregate $>0,63 \mathrm{~mm}$ von $15 \pm 4 \%$ und des SOM-Gehalts von $0,8 \pm 0,3 \%$ auf (Abb.36). Böden dieser Eigenschaften finden sich v.a. in der zentralen Zone, sind aber auch im Chaco und auf dem brasilianischen Schild ausgebildet. Mit steigendem Gewichtsanteil der Aggregate und abnehmenden Gewichtsanteil der Feinsandfraktionen reduziert sich das Erosionsrisiko, ist aber im Bereich von $59 \pm 0,3 \%$ (fS $+\mathrm{ffS})$ und $21 \pm 4 \%(\mathrm{~A}>0,63 \mathrm{~mm})$ noch sehr hoch. Der mittlere Humusgehalt variiert zwischen den Erodibilitätsklassen K1-K4 kaum. Bei Gewichtsanteilen von $50 \pm 4 \%$ (fS + ffS) bzw. $28 \pm 5 \%(\mathrm{~A}>0,63 \mathrm{~mm})$ sind die Böden noch mäßig und bei etwa $43 \pm 6 \%$ (fS + ffS) bzw. $36 \pm 7 \%(\mathrm{~A}>0,63 \mathrm{~mm})$ nur noch gering erodierbar. Übersteigt der Gewichtsanteil der Aggregate $>0,63 \mathrm{~mm} 50 \%$ und sinkt der Gewichtsanteil der Feinsandfraktionen auf unter $30 \%$, sind die Böden von ihren natürlichen Eigenschaften her kaum noch winderosionsgefährdet (Abb.36).

In regionaler Hinsicht wurde eine allgemeine Abschätzung des potenziellen Winderosionsrisikos auf Grundlage der Textur und der Topographie vorgenommen. Grundsätzlich besteht die höchste potenzielle Gefährdung bei sandigen Texturen und im flachen Gelände. Auf Grundlage der Ergebnisse der Bodenartenregionalisierung und über den Reliefparameter Höhe über Tiefenlinie [AD] (Kap.3.3, Abb.10) wurden potenziell gefährdete Bereiche ausgewiesen. Auf Basis von [AD] wurden die Bereiche innerhalb der Alluvialebene selektiert, die dem Wind die günstigsten Anströmbedingungen bieten. Dies sind z.B. flache Kuppen, die aufgrund ihrer exponierten Position einer hohen potenziellen Erosisongefahr unterliegen. Aus 
der Kombination beider Parameter leitet sich die potenziell höchste Gefährdung für Böden mit hohen Sandanteilen und einer leicht exponierten Lage innerhalb der Alluvialebene ab (Abb.37). Deutlich wird, dass innerhalb der ackerbaulich genutzten Regionen die zentrale Zone, insbesondere im Bereich der südwestlichen Alluvialebene, die höchste potenzielle Gefährdung besitzt. In der Expansionszone nimmt die potenzielle Gefährdung aufgrund der Dominanz feinkörniger Bodenarten ab. Insgesamt ist die Chacoregion am stärksten von möglicher Winderosion betroffen, diese Bereiche spielen aber in ackerbaulicher Hinsicht keine Rolle.

\subsubsection{Einfluss des Landnutzungssystems}

Für die Einschätzung des Winderosionsrisikos in Bezug auf die Landnutzungssysteme spielen die spezifischen Bodenbearbeitungspraktiken, die Größe der Anbauflächen und mögliche Schutzsysteme eine wesentliche Rolle.

Mechanisierte Bodenbearbeitung führt zu einem verstärkten Zerfall von Aggregaten und erhöht darüber den Anteil mobiler Bodenpartikel, die vom Wind transportiert werden können (GoMes et al. 2003). Mit diesem Prozess geht eine stärkere Abtrocknung an der Bodenoberfläche einher und macht diese anfällig gegenüber Winderosion. Sind keine Brachen eingeschaltet, wie in dem System der Mennoniten üblich, verstärkt sich der Austrocknungseffekt zusätzlich, da Feuchtigkeit nicht in ausreichendem Maß im Boden gespeichert werden kann. Diese Problematik erhöht sich durch das periodische Auftreten von Trockenperioden (Kap.2.2.1). Pflugbearbeitung ist bei allen praktizierten Betriebssystem üblich, demnach ein höheres Gefährdungspotenzial gegenüber Winderosion eher mit der Quantität der Bearbeitungsschritte einhergeht, da der Zerfall von Aggregaten zusätzlich forciert wird.

Mit steigender Feldgröße erhöht sich durch die größer werdenden ungeschützten Distanzen die Windlauflänge (fetch) und, infolge geringerer Bodenrauigkeit, die Windgeschwindigkeit (ZOBECK et al. 2003). Theoretisch können in Abhängigkeit von der Länge der Anbauflächen solange Partikel aus dem Bodenverband gelöst und transportiert werden, bis die maximale Transportkapazität der Luftströmung erreicht ist (FRYREAR et al. 2001). Dies verdeutlicht, warum besonders auf großen Feldern der Aggregatstabilität als Schutz vor erosiven Winden hohe Bedeutung zukommt. Demnach sind die Anbauflächen der industriellen Landwirtschaft stärker gegenüber Winderosion anfällig, als es in den Gebieten der Kleinbauern der Fall ist. Dort reduziert sich das Risiko durch geringere Schlaggrößen und durch den meist hohen Anteil umgebender Sekundärvegetation, die den Wind stärker abbremst.

Die Anlage von Windschutzstreifen kann die erosive Energie des Windes deutlich reduzieren (PERI \& BlOOMBERG 2002). Während in der Expansionszone die jüngeren Anbau-flächen der Agroindustrie durch breite Windschutzstreifen des ursprünglichen Trockenwaldes eingefasst sind (Abb.6.2), wurden Erosionsschutzpflanzungen an älteren Flächen, insbesondere 


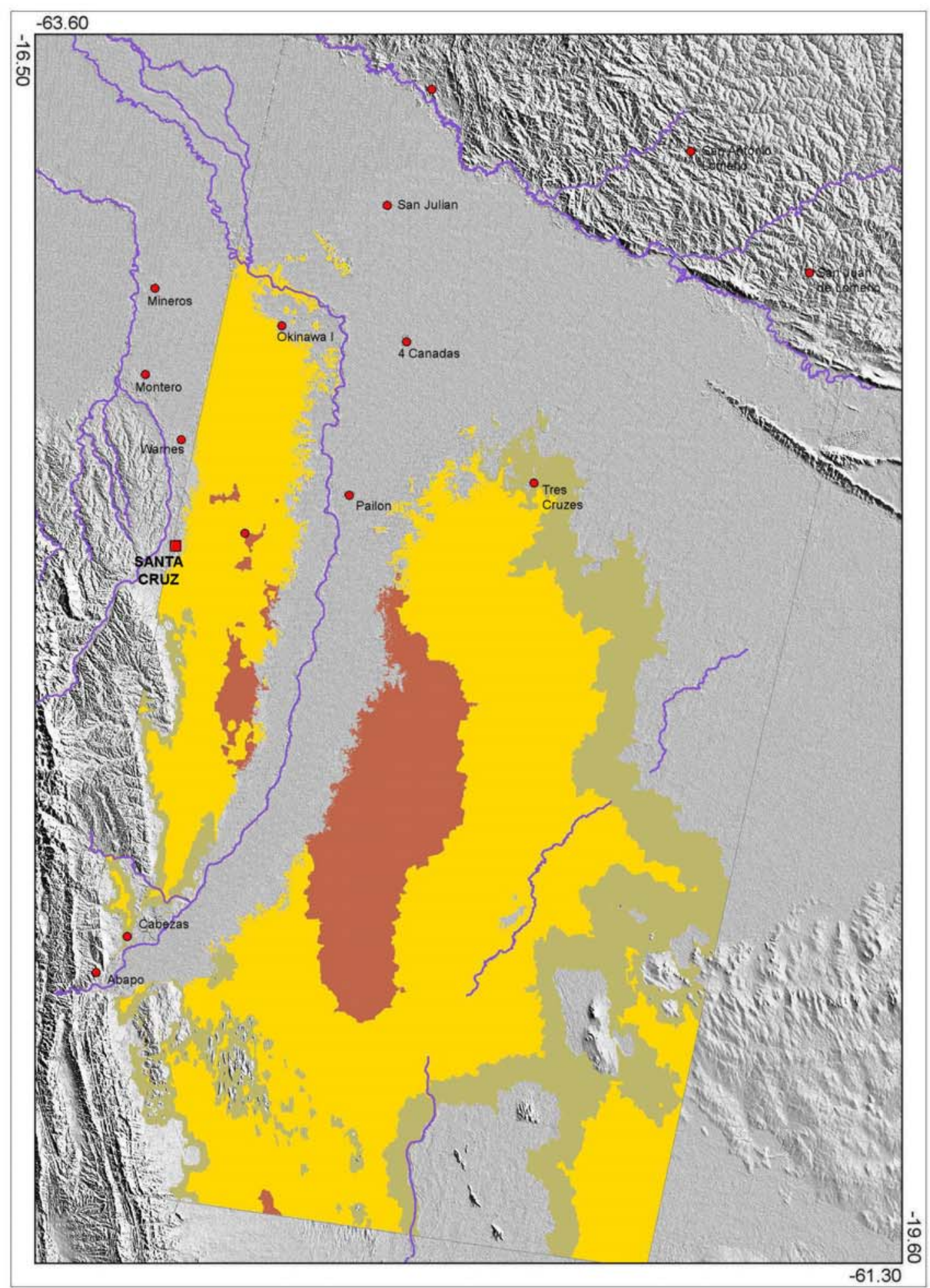

Potential wind erosion risk

(estimated from soil texture and topography information)
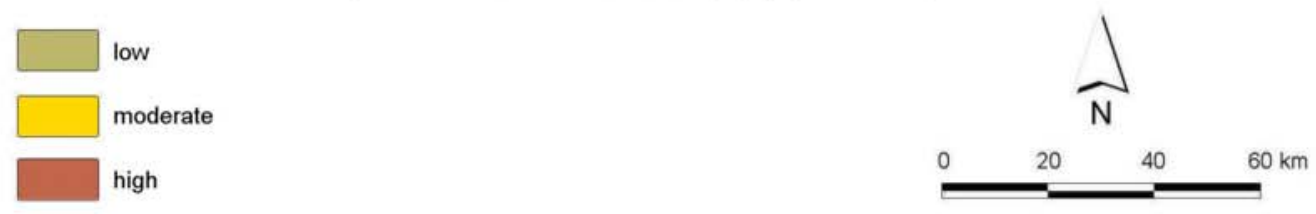

Abb. 37: Potenzielles Winderosionsrisiko auf Grundlage der Bodenart und der Topographie 
in den mennonitischen und japanischen Kolonien, erst im Nachhinein per staatlichem Dekret ausgeführt (Kap.5.1). Da es für die Umsetzung offensichtlich keine Richtlinien gab, ist deren Schutzwirkung z.T. fragwürdig. Um den Verlust an Produktionsfläche gering zu halten, werden meist nur einreihige Windschutzstreifen gepflanzt. Diese sind dann relativ wirkungslos, wenn der Unterwuchs nicht geschlossen ist oder diese größere Lücken aufweisen (Abb.38). Im Bereich von Lücken konzentriert sich der Luftstrom, was zu einem Anstieg der Windgeschwindigkeit im Leebereich der Windschutzstreifen führt. Sind die Felder, wie bei älteren Anbauflächen häufiger anzutreffen, längs zur Hauptwindrichtung orientiert, bieten Windschutzstreifen keinen Schutz. Vielmehr kann es dort zu einer Verstärkung der Windgeschwindigkeit kommen. Maximalen Schutz bieten Windschutzstreifen, verlaufen diese quer zur Windrichtung (WILKINSON \& ELEVITCH 2000). Entsprechend der im Untersuchungsraum vorherrschenden Hauptwindrichtung sollten Windschutzstreifen in SW-NE Orientierung angelegt sein.
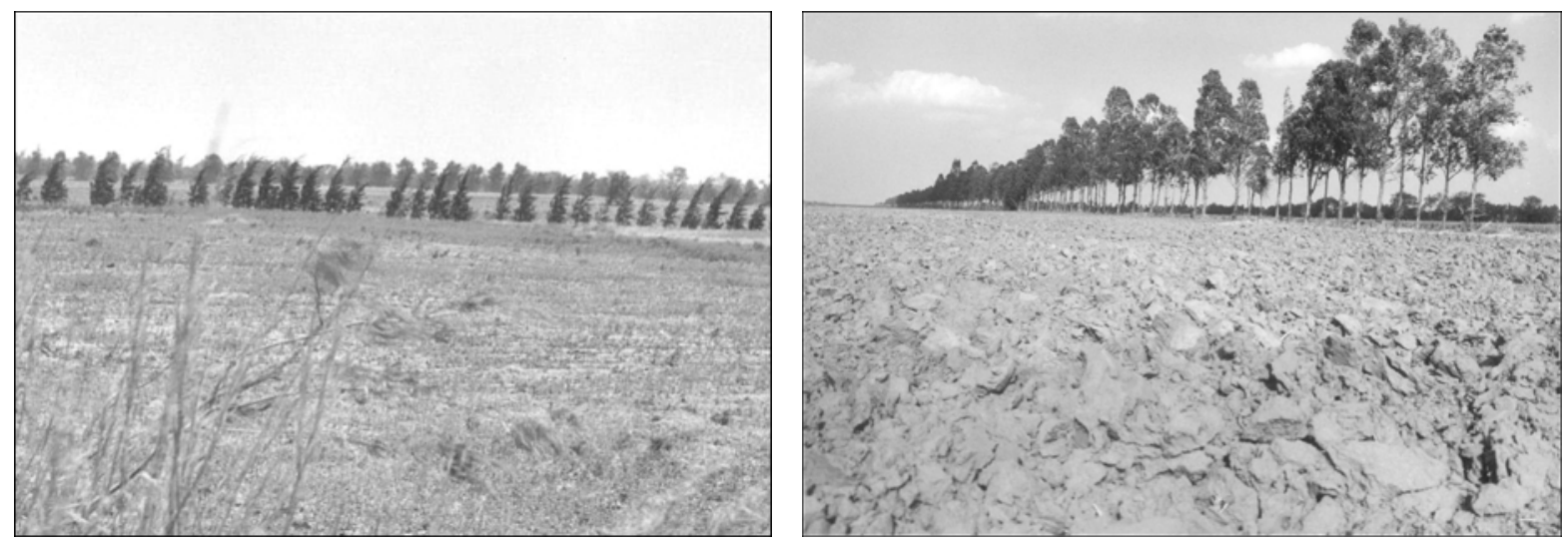

Abb. 38: Windschutzstreifen in der Mennonitenkolonie Riva Palacios

Deutlich wird, dass das höchste Gefährdungspotenzial auf den Flächen der industrialisierten Landwirtschaft besteht (Agroindustrie, Mennoniten, Japaner). Begründet wird die Annahme durch die mechanisierte Bewirtschaftungsweise und die z.T. beträchtlichen Feldgrößen. In Kombination mit den pedologischen Eigenschaften (hohe Feinsandanteile) und den klimatischen Faktoren (semi-arid, hohe Windgeschwindigkeiten) besteht regional das höchste Winderosionsrisiko in der südlichen zentralen Zone (Las Brechas). Im Vergleich mit der nördlichen zentralen Zone determinieren einzig die geringeren Niederschläge und damit verbunden geringeren Bodenfeuchten die höhere Anfälligkeit. Für die Expansionszone wird das Risiko geringer bewertet, da die mittleren Windgeschwindigkeiten abnehmen und die pedologischen Eigenschaften eine stärkere Aggregierung im Boden bewirken. Zudem reduzieren mächtige Windschutzstreifen in den Gebieten der Agroindustrie das Risiko potenzieller Bodenverluste durch Wind. In den nördlichen Anbauzonen ist die Wahrscheinlichkeit von Erosionsschäden durch Wind aufgrund der geringern Windgeschwindigkeiten, der überwiegend feinkörnigen Substrate und der humideren Bedingungen gering. 
Insgesamt steigt das Erosionspotenzial durch die windoffene, ebene Lage der Anbauflächen in der Alluvialebene. Zwar sind auch auf dem brasilianischen Schild und im Subandin Böden mit hohen Feinsandanteilen ausgebildet, aber durch die geneigte Topographie verringert sich die potenziell vom Wind erodierbare Fläche, ebenso wie durch die umgebende Vegetation die Windgeschwindigkeit. Zusammen mit kleinen Parzellengrößen und humideren Bedingungen (brasilianischer Schild) ist das Gefährdungspotenzial in diesen Gebieten gering. Morgenroth (1999) kommt in einer Studie zur Bodenerosion zu dem Ergebnis, dass die Faktoren Aridität (Zunahme) und Hangneigung (Abnahme) wesentlichen Einfluss auf den Anteil winderodierter Fläche haben.

\subsubsection{Zusammenfassung}

In klimatischer Hinsicht steigt das Risiko winderosiver Prozesse mit zunehmender Trockenheit, weshalb das Risiko potenzieller Winderosionsschäden im semi-ariden Süden am größten ist. Das Risiko steigt mit zunehmender Schlaggröße und ist somit in den Gebieten der Agroindustrie und der Mennoniten am ausgeprägtesten, in den Mennonitenkolonien zusätzlich verstärkt durch die z.T. ungenügende Windschutzbepflanzung. Die höchsten Windgeschwindigkeiten mit Spitzenwerten von über $20 \mathrm{~m} \mathrm{~s}^{-1}$, treten in der zentralen Zone auf. Dort liegt der Anteil potenziell erosiver Windgeschwindigkeiten jährlich bei $14 \%$, in der Trockenzeit bei $21 \%$. Im Norden sind die Windverhältnisse moderater, nur noch $2-4 \%$ der Winde erreichen im Jahresverlauf potenziell erosive Geschwindigkeiten (5-8 \% in der Trockenzeit). An ausgeglichensten stellen sich die Windverhältnisse im Osten dar. Dort erreichen lediglich $1 \%$ der Winde Geschwindigkeiten, die potenziell erosive Prozesse auf den Böden nach sich ziehen können. Von den pedologischen Eigenschaften her sind Böden mit Anteilen von über 60 Gew.- $\%$ in den Feinsandfraktionen, einem Aggregatanteil $(>0,63 \mathrm{~mm})$ von unter 15 Gew.-\% und einem SOM-Anteil von weniger als 1 Gew.-\% am leichtesten vom Wind erodierbar. Dies umfasst ausschließlich die sandigen Lehme und lehmigen Sande. Einen direkten Einfluss auf die Erodibilität haben die Bodenart und die Aggregierung. Die organische Substanz wirkt vor allem sekundär über die Aggregatbildung im Boden. Bei Feinsandgehalten von unter 30 Gew.-\% und einem Anteil der Aggregate von über 50 Gew.-\% sind die Böden nur noch sehr gering vom Wind erodierbar. Regional ist die zentrale Zone durch die dort auftretenden hohen Windgeschwindigkeiten und von den Bodeneigenschaften her am stärksten gefährdet. Bei den feinkörnigeren Böden der Expansionszone besteht ein potenzielles Erosionsrisiko dann, wenn Bodenaggregate durch Bearbeitungsprozesse auf eine Größe zerfallen, die leicht vom Wind transportiert werden kann. 


\section{DISKUSSION UND AUSBLICK}

Die übergeordnete Fragestellung, ob sich Bodendegradationsprozesse auf der Grundlage qualitativ heterogener Bodendaten unter Einbezug von Hilfsvariablen (sekundäre Geodaten, Literaturangaben) räumlich quantifizieren lassen, kann grundsätzlich mit ja beantwortet werden. Mögliche Restriktionen die bei der Umsetzung auftauchten, werden im Anschluss an die Ergebnisdiskussion erläutert.

In Anbetracht der Ergebnisse dieser Studie, kann der von DERPSCH (2000) eingangs erwähnten Stellungnahme zugestimmt werden, dass konventionelle Bodenbearbeitung, wie sie mehrheitlich im Untersuchungsgebiet betrieben wird, eine für tropische Breiten hochgradig degradierende Form der Bewirtschaftung ist. Dabei stehen die in diesem Zusammenhang geschilderten Prozesse (Verlust an organischer Substanz, Bodenverdichtung, Winderosion) in gegenseitiger Wechselwirkung zueinander. Bodenbearbeitung fördert die Durchlüftung und führt zu einem Anstieg der Bodentemperatur, mithin zu einem beschleunigten Abbau der organischen Substanz. Mit der Reduktion organischer Substanz vermindert sich die Stabilität der Bodenaggregate. Gleichzeitig steigt die Disposition eines Bodens zu verdichten bzw. infolge des Aggregatzerfalls der Anteil an mineralischen und organischen Bodenpartikeln die leicht vom Wind erodiert werden können (Abb.1).

Aus den Ergebnissen wird deutlich, dass die, aufgrund der längeren Bewirtschaftungszeit, aktuell am stärksten von Bodendegradation betroffene Region, die zentrale Zone ist. Während Verdichtungen in allen Böden auftreten können, betrifft die Nährstoffverarmung durch den Verlust an organischer Bodensubstanz v.a. sandige Substrate. Auf diesen Böden wiederum besteht die höchste potenzielle Winderosionsgefährdung. Das mit der gängigen Bewirtschaftungsweise mittelfristig Ertragverluste bis hin zur Aufgabe von Flächen einhergehen, wie dies aktuell in der zentralen Zone zu beobachten ist, scheint bei den Entscheidungsträgern bislang wenig Beachtung zu finden, trotzdem in zahlreichen Publikationen wiederholt auf diese Problematik hingewiesen wurde (z.B. BARBER 1995, BARBER et al. 1996, DAVIES 1996, Gerold 1986, 2001b, 2002a, PACHeCo 1998, Thiele \& BARber 1998, Urioste \& PACHeCO 2001). Der fortschreitende Verlust landwirtschaftlicher Produktionsflächen hat für Bolivien dramatische Folgen, bedeutet es einerseits ausbleibende Devisen und anderseits eine stärkere Abhängigkeit gegenüber Importen. Gleichzeitig werden vermehrt marginale Standorte in Kultur genommen, da produktive Agrarflächen aufgrund der klimatischen und topographischen Verhältnisse im Land rar sind (URIOSTE \& PACHECO 2001).

Vor dem Hintergrund der Ergebnisse dieser Studie sowie der ökonomischen Rahmenbedingungen taucht somit die Frage nach Bewirtschaftungsalternativen auf, die imstande sind, die Bodenfruchtbarkeit und somit die Produktivität der aktuell genutzten Flächen langfristig $\mathrm{zu}$ sichern. 


\section{Ergebnisse}

Die Ergebnisse der Landnutzungsanalyse belegen die außergewöhnliche Entwicklungsdynamik im ostbolivianischen Tiefland in den letzten zwei Jahrzehnten. Gravierende Ausmaße der Konversion ursprünglicher Wälder in Agrarflächen gehen auf die 1990er Jahre zurück. Mit der Inkulturnahme der Expansionszone gehen Rodungsraten einher, die im nördlichen Untersuchungsgebiet mit 6,5 \% jährlich weltweit mit zu den höchsten gehören. Im 17-jährigen Untersuchungszeitraum hat sich die Primärwaldfläche dieses Raums um annähernd die Hälfte reduziert. Im Vergleich dazu liegen die von ACHARD et al. (2002) ermittelten Raten in stark von Abholzung betroffenen tropischen Regionen (hot spots) zwischen 0,9-5,9 \% (Tab.41). Wie gezeigt werden konnte, unterliegt die landwirtschaftliche Expansion im südlichen Untersuchungsgebiet klimatischen Restriktionen. Folglich befinden sich die Rodungsraten im gleichen Zeitraum auf einem niedrigeren Niveau, liegen aber mit 0,9 \% noch immer über dem südamerikanischen Durchschnitt von 0,4 \% (FAO 2003).

Tab. 41: Jährliche Abholzungsraten in ausgewählten tropischen Regionen mit hoher Waldkonversion (Bezug Waldfläche 1990)

\begin{tabular}{cc}
\hline Region & jährliche Abholzungsrate \\
\hline Brasilien, Amazonasraum & $0,9-4,4 \%$ \\
Madagaskar & $1,4-4,7 \%$ \\
Süd-Vietnam & $1,2-3,2 \%$ \\
Zentral Sumatra & $3,2-5,9 \%$ \\
\hline
\end{tabular}

Quelle: ACHARD et al. (2002), verändert

Treibende Kräfte der jüngeren Entwicklung sind staatliche Strukturanpassungsprogramme, mittels derer die in den 1980er Jahren in einem desolaten Zustand befindliche bolivianische Wirtschaft gefördert werden sollte (PACHECO 2001). Wesentlicher Bestandteil war der Ausbau des Agrarsektors in Richtung einer devisenbringenden, exportorientierten Landwirtschaft. Demzufolge sind rund zwei Drittel der im Untersuchungszeitraum festgestellten Rodungen der Expansion agroindustrieller Großbetriebe zuzuordnen. Konstant hoch sind die Abholzungsratensraten auch in den Gebieten der Kleinbauern, auf die etwa ein Fünftel der Waldkonversion zurückgeht. Waren es bis in die 1980er Jahren hinein vornehmlich die geplanten Kleinbauernkolonien, die in dieser Nutzergruppe expandierten, so zeigt sich in den 1990er Jahren ein starker Zuwachs an spontaner Kolonisation. Diese Entwicklung geht darauf zurück, dass mit Umsetzung der staatlichen Entwicklungspläne die seit Mitte der 1950er Jahre bestehenden staatlichen Programme zur Ansiedlung von Kleinbauern in Kolonien aufgegeben wurden (KAIMOWITZ \& SMITH 2001). Stattdessen siedelten sich in den neu erschlossenen Gebieten verstärkt spontane Kolonisten an. Die dritte große Nutzergruppe sind die Mennoniten, deren Anteil an der gerodeten Fläche konstant bei $15 \%$ liegt. 
Als Folge der starken landwirtschaftlichen Expansion in den 1990er Jahren wird knapp die Hälfte der 2001 ackerbaulich genutzten Flächen erst seit etwa 10 Jahren kontinuierlich bewirtschaftet. Knapp $16 \%$ weisen eine kontinuierliche Anbaunutzung von 10-20 Jahren und $13 \%$ von mehr als 20 Jahren auf. Bei ca. $4 \%\left(\sim 670 \mathrm{~km}^{2}\right)$ der langjährig genutzten Flächen handelt es sich um aufgelassene Anbauflächen, die allenfalls noch extensiv beweidet werden. Die Auflassung der Flächen ist eine Folge zunehmender Bodendegradation, wodurch ein produktiver Anbau auf diesen nicht mehr möglich ist. FEARnsIDE (2001) sieht diese Entwicklung als wesentlichen Grund für die Aufgabe der ältesten Mennonitenkolonien in der älteren Landwirtschaftszone (zentrale Zone) und deren Neuansiedlung in der Expansionszone an. Die von ihm angegebene ungefähre Größenordnung von $1.000 \mathrm{~km}^{2}$ aufgelassener Anbauflächen Ende der 1990er Jahre entspricht dem in dieser Studie ermitteltem Niveau, da auch Bereiche außerhalb des Untersuchungsgebiets der vorliegenden Studie berücksichtigt wurden.

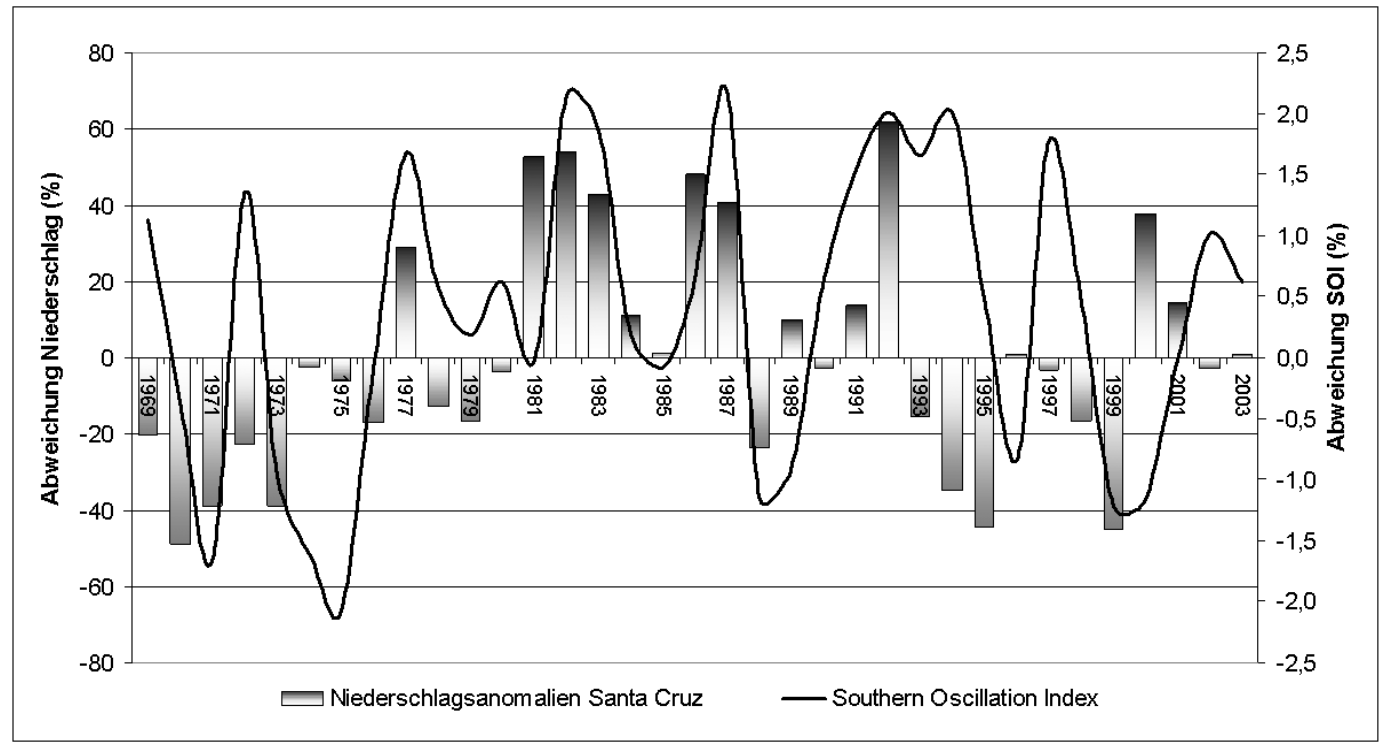

Quelle: KEMP (2005)

Abb. 39: Beziehung zwischen jährlichem Niederschlag (Station EI Trompillo, Santa Cruz) und Southern Oscillation Index (SOI)

Dass die zunehmende Konversion tropischer Wälder in Agrarflächen regionale Auswirkungen auf das Klima hat, wird über Modellrechnungen in der Regel bestätigt (LAWTON et al. 2001, ZHANG et al. 2001). Kontrovers diskutiert werden dagegen die möglichen globalen Effekte (BAZZAZ 1998, CrAMER et al. 2004, ZHAO et al. 2001). Die Ursache möglicher regionalklimatischer Veränderungen ist auf die Reduktion der effektiven Verdunstungsfläche zurückzuführen. Insgesamt kann weniger Strahlung durch die Vegetation absorbiert werden, wodurch ein höherer Strahlungsinput an der Bodenoberfläche auftritt. Der fühlbare Wärmestrom erhöht sich, während der latente abnimmt. Die geringeren Evapotranspirationsraten bedeuten, dass weniger Wasserdampf der Atmosphäre zugeführt wird und sich folglich die potenziellen Niederschlagsmengen reduzieren. Modellsimulationen kommen mehrheitlich zu 
dem Ergebnis, dass das regionale Klima in tropischen Breiten trockener und wärmer wird (LEAN \& RownTREE 1997). Für den Amazonasraum simulieren die meisten Modelle lokale Abnahmen der Niederschlagsmengen um 220-640 $\mathrm{mm} \mathrm{a}^{-1}$, der Evaporation um 164-550 mm $\mathrm{a}^{-1}$ sowie einen Anstieg der bodennahen Temperatur um 0-3 ${ }^{\circ} \mathrm{C}$ (WERTH \& AVISSAR 2002).

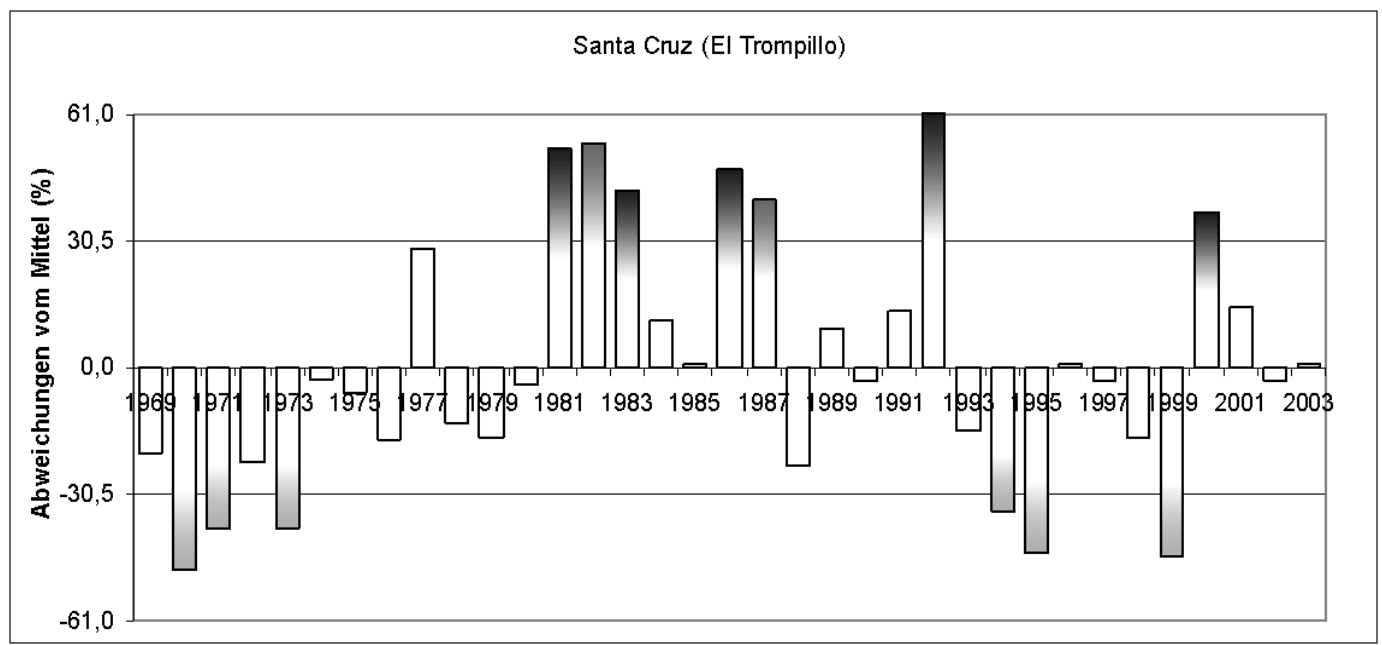

Quelle: KEMP (2005)

Abb. 40: Häufigkeiten extrem trockener bzw. extrem feuchter Jahre (Station El Trompillo, Santa Cruz) (Variationskoeffizient 30,5\%, positive Abweichungen $=$ feuchte Jahre, negative Abweichungen $=$ trockene Jahre)

Für das Untersuchungsgebiet ermittelten BouNOUA et al. (2003) als Folge der großflächigen Abholzungen einen Anstieg der mittleren Monatstemperatur im Januar von etwa $0,5^{\circ} \mathrm{C}$. Dieser Trend wird über Messdaten bestätigt. Verbunden mit der allgemeinen Temperaturerhöhung sind bis $\mathrm{zu} 2^{\circ} \mathrm{C}$ höhere Tagesmaxima sowie eine stärkere Abkühlung in der Nacht. Bezüglich der Niederschläge konstatiert KEMP (2005) einen allgemeinen Rückgang der Niederschlagssummen seit Ende der 1980er Jahre und stellt dies in einen möglichen Zusammenhang mit der Abholzung. Als denkbares Indiz einer regionalen Ursache führt er an, dass die zeitliche Verteilung der Niederschläge in Santa Cruz (Messperiode 1969-2003) in keinem korreliertem Zusammenhang mit La Niña Ereignissen steht (Abb.39). Von diesen wird angenommen, dass sie im westlichen Südamerika trockene Perioden induzieren (KANE 1999). Gegen die Theorie würde sprechen, dass bereits in den 1970er Jahren, einer Zeit mit weit mehr Waldfläche als zum heutigen Zeitpunkt, eine vergleichbar trockene Phase aufgezeichnet wurde. Zudem sind die regionalen Auswirkungen von ENSO-Ereignissen in Südamerika bisher nicht ausreichend erforscht (COELHO et al. 2002). Interessant jedenfalls wäre, diesen Aspekt anhand von Klimamodellsimulationen näher zu überprüfen. Deutlich wird aus den Analysen von KEMP (2005), dass sich in den letzten Jahrzehnten eine tendenzielle Zunahme extrem trockener bzw. extrem feuchter Perioden abzeichnet (Abb.40). Diese Tatsache wird auch über Untersuchungen von BARBER (1995) bestätigt. Welche Gründe für diese Entwicklung verantwortlich sind, ist bis dato unklar. 
Die mit der Abholzung einhergehende Erwärmung forciert die mikrobielle Aktivität und dadurch die Mineralisation der organischen Substanz im Boden. Werden die gerodeten Flächen ackerbaulich genutzt, wird der Abbau durch die Bodenbearbeitung zusätzlich verstärkt. Im Untersuchungsgebiet gelten speziell bodenwendende Maßnahmen (Wendepflug) und die Zerstörung von Bodenaggregaten (Scheibenegge) in Kombination mit einer häufig unbedeckten Bodenoberfläche als ursächlich für den forcierten Abbau organischer Substanz (BARBER et al. 1996, BARBER 1995). Dass dieser Prozess vergleichsweise schnell abläuft, konnte über die Ergebnisse bestätigt werden. Die dem Boden durch Ernterückstände zugeführte organische Substanz kann die potenziellen Verluste im konventionellen System nicht kompensieren (SCHLESINGER \& ANDREWS 2000), zumal es im ostbolivianischen Tiefland gängige Praxis ist, diese abweiden zu lassen bzw. zu verbrennen. Dazu bemerkt KATYAL (2001): „In tropical areas, the practice of returning residues to the soil is practically nonexistent".

Die Verluste im Oberboden liegen sowohl für SOC als auch für $\mathrm{N}_{\mathrm{t}}$ bereits nach wenigen Jahren in der Größenordnung von einem Drittel. Nach mehr als 20-jähriger Bewirtschaftungsdauer haben sich die Vorräte um etwa die Hälfte reduziert. Das gilt für SOC und $\mathrm{N}_{t}$ gleichermaßen. Generell verläuft der Abbau mit steigendem Tongehalt langsamer. Dieser Unterschied verringert sich mit zunehmender Kultivierungsdauer, so dass sich die relativen Verluste nach langjähriger Nutzung auf einem vergleichbaren Niveau befinden. Während der Vorratsabbau unter konventioneller Bodenbearbeitung sehr schnell abläuft, benötigt die Wiederherstellung des Ausgangsniveaus oft sehr lange Zeiträume. Im globalen Mittel beträgt die Kohlenstoffbindung im Boden nach landwirtschaftlicher Nutzung zwischen 0,3-0,6 $\mathrm{t} \mathrm{ha}^{-1} \mathrm{a}^{-1}$ (HAMBURG 2000, Post \& KWON 2000, WBGU 2003). Für warm-trockene Klimate geht LAL (2002) von einer Größenordnung zwischen 0,1-0,3 t C ha ${ }^{-1} \mathrm{a}^{-1}$ aus. Dass diese Werte sehr unterschiedlich sein können, belegen Untersuchungen von BURKE et al. (1995) ${ }^{28}$, die auf aufgelassenen Anbauflächen in der semi-ariden Kurzgrassteppenzone der USA eine Akkumulationsrate von lediglich 0,03 t C ha ${ }^{-1} \mathrm{a}^{-1}$ ermittelten (50-jähriges Mittel). Auf den aufgelassenen Anbauflächen der zentralen Zone würde, bei einem angenommenen SOC-Verlust von $20 \mathrm{tha} \mathrm{ha}^{-1}$ (Tab.31), die Wiederherstellung des Ausgangsniveaus, unter Annahme einer hypothetischen Kohlenstoffbindung von $0,1 \mathrm{t} \mathrm{ha}^{-1} \mathrm{a}^{-1}$, etwa 200 Jahre benötigen. Allerdings ließe sich die Kohlenstoffbindung in degradierten Böden durch die Etablierung geeigneter Gräser und ein gezieltes Weidemanagement erhöhen (BATJES 1998, LAL 2003).

Die höchsten relativen Verluste organischer Substanz sind auf den sandigen Lehmen der zentralen Zone zu verzeichnen. Die Böden können z.T. nach wenigen Anbaujahren mit SOMVorräten von unter $30 \mathrm{t} \mathrm{ha}^{-1}(0-20 \mathrm{~cm})$ als humusarm eingestuft werden. Überdies sind diese Böden am stärksten winderosionsgefährdet, wobei das Risiko mit zunehmender Flächengröße und abnehmender Bodenrauigkeit steigt und demzufolge in den Gebieten industrieller Landwirtschaft (Agroindustrie, Mennoniten, Japaner) am höchsten ist. Dagegen befinden sich die

${ }^{28}$ zitiert in POST \& KWON (2000) 
SOM-Vorräte tonreicherer Böden durchweg auf einem höheren absoluten Niveau, woraus sich theoretisch ein produktiver Anbau über einen vergleichsweise längeren Zeitraum ableitet. DAVIES (1996) kommt in einer ökonomischen Modellrechnung für eine repräsentative agroindustrielle Farm der Expansionszone zu dem Ergebnis, dass diese unter den gegebenen Bedingungen $^{29} 1992$ etwa 10 Jahre höchst profitabel wirtschaftet, in der Folge aber eine merkliche Reduktion des Nettogewinns durch einen sukzessiven Anstieg der Produktionskosten und sinkende Erträge zu verzeichnen ist. Nach 20-25 Jahren kontinuierlicher Nutzung kann noch mit ausreichenden Renditen gerechnet werden, obwohl der hypothetische Anteil brachgefallener Anbauflächen (extensive Weide) stetig wächst. Vor diesem Hintergrund könnte in der Expansionszone noch einige Zeit profitabel gewirtschaftet werden, vorausgesetzt die Weltmarktpreise bleiben stabil. Allerdings deuten die hohen relativen SOC- und $\mathrm{N}_{\mathrm{t}}$-Verluste an (Tab.31), dass bei Fortdauer der gängigen Bewirtschaftungsweisen auch auf den Böden der Expansionszone die Fertilitätsverluste mittelfristig eine Größenordnung erreichen, in deren Folge mit Ertragseinbußen zu rechnen ist. Diese Annahme ist insofern berechtigt, da in der Regel weder Dünger appliziert werden (BARBER \& NAVARRO 1994b), noch über die Ernterückstände der cash-crops, insbesondere bei Soja, eine nennenswerte SOM-Anreicherung im Boden erfolgt, die der raschen mikrobiellen Zersetzung entgegenwirken könnte (BARBER 1995, WEST \& POST 2002). Nicht eingerechnet in der Studie von DAVIES (1996) sind mögliche Ertragsverluste durch Bodenverdichtungen. Wie gezeigt wurde, sind unter mechanisierter Bodenbearbeitung in allen Böden des Untersuchungsgebiets Verdichtungen möglich, indes ist das Risiko schadhafter Verdichtungen in der Expansionszone, und dort in den Gebieten der agroindustriellen Farmen, am höchsten.

Die Ergebnisse verdeutlichen, dass der Aufrechterhaltung der SOM-Vorräte für den Erhalt der Bodenproduktivität eine Schlüsselrolle zukommt. Auf diesen Aspekt hat bereits GEROLD (1986) in einer Studie über die Bodennutzungsprobleme im Tiefland von Santa Cruz hingewiesen. Das Ziel muss demnach eine Reduktion der Mineralisationsraten bei gleichzeitiger Anreicherung der organischen Substanz im Boden sein. Dies ist letztlich nur über einen erhöhten Biomasseeintrag zu erreichen (MATSON et al. 1997). Gleichzeitig sollte die Bearbeitungshäufigkeit verringert werden, um das Risiko von Bodenverdichtungen zu minimieren. Dazu bieten sich Verfahren der konservierenden Bodenbearbeitung an, die sich von den konventionellen Praktiken durch eine in der Folge abnehmende Bearbeitungsintensität unterscheiden: reduzierte Bodenbearbeitung (reduced tillage), Minimalbodenbearbeitung (minimum till) und Direktsaat (no-till) (MACHADO \& SILVA 2001). Werden bei den erstgenannten Verfahren noch Bodenbearbeitungen durchgeführt, wird bei der Direktsaat das Saatgut direkt in den unbearbeiteten Boden eingebracht (PAUSTIAN et al. 2000). Verfahren der konservierenden Bodenbearbeitung (vorwiegend Direktsaat) wurden in anderen Ländern, als

\footnotetext{
${ }^{29}$ konventionelle Bodenbearbeitung, Landnutzung (Soja, Mais, Weizen), Nutzung von Agrochemikalien, keine Düngerapplikationen, Weltmarktpreise 1992
} 
Reaktion auf die fortschreitende Bodendegradation und nachlassender Erträge, z.T. schon vor längerer Zeit eingeführt. Länder wie die USA (z.B. AL-KAISI et al. 2005, FrANZLUEBBERS 2002, SiX et al. 1999, TAN \& LAL 2005, URI 2000) und Brasilien (z.B. BAYER et al. 2001, CASTRO et al. 1999, LANDERs 2001, MACHADO \& Silva 2001, SÁ et al. 2001) besitzen Schätzungen zufolge die höchsten Flächenanteile, auf denen Direktsaat in der industriellen Landwirtschaft praktiziert wird (DERPSCH 2001).

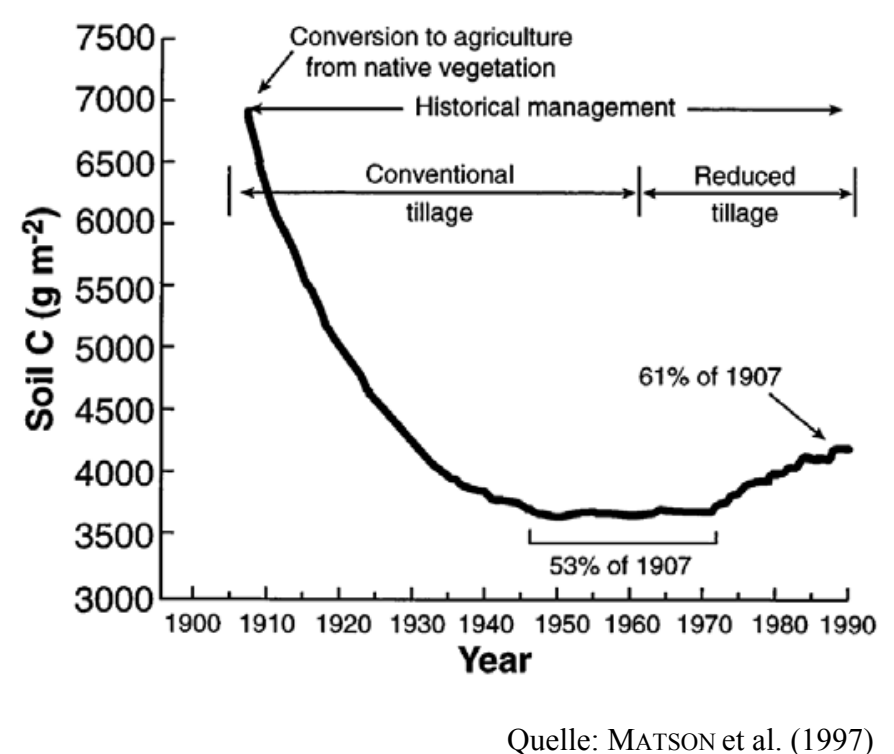

Abb. 41: Änderung des SOC-Vorrats nach Umstellung von konventioneller auf reduzierte Bodenbearbeitung (0-20 cm)

Gemeinsam ist den Verfahren der konservierenden Bodenbearbeitung, dass die Bodenoberfläche im Jahresverlauf permanent bedeckt ist (Mulch), z.B. mit Ernterückständen (Bedeckungsgrad $>30 \%$ ) oder durch die Pflanzung von Zwischenfrüchten (Bodenbedecker). Eine Mulchschicht reduziert die Verdunstung und erhöht die Feuchtigkeit im Boden, die Kulturen sind folglich weniger anfällig für Trockenstress. Zudem verringert sich die Bodentemperatur und somit der Abbau organischer Substanz (LAL \& KIMBLE 1997, PAUSTIAN et al. 2000, REICOSKY 1997). Schließlich reduziert sich durch die höhere Bodenrauigkeit das Winderosionsrisiko (GOMES et al. 2003).

Neben dem rein physikalischen Schutz vor Witterungseinflüssen und somit vor Erosion, erhalten bodenschonende Anbaupraktiken langfristig die Bodenfruchtbarkeit und führen bei richtigem Management (angepasste Fruchtfolgen, Gründüngung) zu einer Anreicherung organischer Substanz im Boden (MACHAdO \& Silva 2001). WeST \& PosT (2002) kommen in ihrem Review zu dem Ergebnis, dass durch die Umstellung von konventioneller Bodenbearbeitung auf Direktsaatverfahren im Mittel $57 \pm 14 \mathrm{~g} \mathrm{C} \mathrm{m}^{-2} \mathrm{a}^{-1}$ im Boden gebunden werden können. Dieser Anstieg ist etwa 5-10 Jahre nach Umstellung nachweisbar (Abb.41). Schon die Umstellung auf standortgerechte Fruchtfolgen hätte ihrer Analyse nach das Potenzial, 
jährlich $20 \pm 12 \mathrm{~g} \mathrm{C} \mathrm{m}^{-2} \mathrm{a}^{-1}$ im Boden anzureichern $(\sim 0-15 \mathrm{~cm})$. In der gleichen Größenordnung liegen die von SÁ et al. (2001) in Südbrasilien ermittelten Werte, die unter Direktsaatverfahren eine SOC-Anreicherung von $81 \mathrm{~g} \mathrm{~m}^{-2} \mathrm{a}^{-1}(0-20 \mathrm{~cm})$ konstatieren.

MACHADO \& Silva (2001) betrachten Verfahren der konservierenden Bodenbearbeitung wegen der häufig extremen Wetterbedingungen in tropischen Breiten aus den genannten Gründen (Erosionsschutz, Erhalt der Bodenfruchtbarkeit) als geeignete Bewirtschaftungsalternative. Auch FrANZLUEBBERS (2002) kommt zu dem Schluss, dass sich konservierende Bodenbearbeitung besonders für den Anbau unter feucht-warmen Klimabedingungen sowie auf Böden geringer SOM-Gehalte eignet. Den häufig zitierten Nachteilen bodenschonender Anbaussysteme, insbesondere der Direktsaat, wie erhöhtes Aufkommen von Pflanzenschädlingen und -krankheiten, kann nach DERPSCH (2002) durch die korrekte Rotation der Anbaufrüchte begegnet werden.

Auch im Untersuchungsgebiet sind in jüngster Zeit Tendenzen erkennbar, Verfahren der konservierenden Bodenbearbeitung in der industriellen Landwirtschaft einzuführen (IADB 2000, URioste \& PACHECO 2001). 1995 wurden im Department Santa Cruz etwa 29.000 ha Anbauflächen im Direktsaatverfahren bestellt (LLANOS et al. 1996). Nach Schätzungen von DERPSCH (2001) betrug die Direktsaatfläche in ganz Bolivien 1999 ca. 200.000 ha. Angesichts der Tatsache, dass 2001 allein im Untersuchungsgebiet rund 900.000 ha kultiviert wurden (Tab.11), ist der Anteil an Direktsaat bislang noch verschwindend gering. URIOSTE \& PACHECO (2001) nennen als Hauptgrund der geringen Umsetzung die mangelnden Kenntnisse der Produzenten über dieses Anbausystem. Dennoch sprechen die positiven Aspekte konservierender Bodenbearbeitung (Erosionsschutz, Erhalt der SOM-Vorräte, höhere Bodenfeuchte) für sich und werden, vor dem Hintergrund der schnell abnehmenden Bodenproduktivität, im ostbolivianischen Tiefland als Bewirtschaftungsalternativen angesehen, die die Ertragsfähigkeit der Böden langfristig zu sichern imstande sind. Unterstützt wird diese Annahme durch die guten Erfahrungen, die mit diesen Techniken, bei vergleichbaren klimatischen Bedingungen, in den letzten 30 Jahren in der industriellen Landwirtschaft Südbrasiliens gesammelt wurden.

\section{Methodik}

Die in der Zielsetzung formulierte Absicht, existierende, qualitativ heterogene Bodendaten geringer räumlicher Auflösung für eine regionale Analyse der Bodendegradation in Wert zu setzten, konnte mit der Vorgehensweise in dieser Arbeit erreicht werden. Durch die ergänzende Nutzung allgemein verfügbarer Flächendaten (Satelliten-, Relief- und Klimadaten) sowie Angaben aus der Literatur, lassen sich mit Hilfe von Geoinformationssystemen (GIS) und geostatistischer Methoden plausible räumliche Aussagen treffen. Der Vorteil der angewendeten Methodik ist, dass kostenintensive und langwierige Bodenuntersuchungen 
vermieden werden und die ergänzenden Daten in aller Regel problemlos über das Internet zu beziehen sind.

Naturgemäß zeigen großräumige, quantitative Analysen lediglich Tendenzen auf, da mit deren Umsetzung zwangsläufig Generalisierungen einher gehen. Lokal abweichende Größenordnungen sind jedoch in der Regel über die Darstellung der Schwankungsbreiten abgedeckt. Die stärkste Abstraktion in dieser Studie ist die Annahme eines einheitlichen, bodenartenabhängigen Ausgangsniveaus für die Parameter SOC, $\mathrm{N}_{\mathrm{t}}$, und $\mathrm{d}_{\mathrm{b}}$. Die Gründe dafür wurden bereits diskutiert, sollen aber dennoch kurz erwähnt werden. Generell ist die großräumige Schätzung bodenchemischer Eigenschaften über Reliefparameter ein Problem, da deren Variabilitäten auf kurzen Distanzen z.T. sehr hoch sind. Diese werden entweder über die Anzahl der Profilpunkte nicht hinreichend erfasst oder können nur über hochauflösende Geländemodelle abgebildet werden. Dazu bemerken HENDERSON et al. (2005): „Some environmental variables that are useful predictors at less than 25 may not be so at $250 \mathrm{~m}$." Folglich beschränken sich methodische Ansätze in dieser Richtung überwiegend auf kleine Gebiete, wie aus dem Review von MCBRATNEY et al. (2003) ersichtlich wird. CERRI et al. (2004a) bspw. verwendeten 980 Profilpunkte und ein DGM mit 25 m Rasterweite, um die räumliche Variabilität bodenchemischer Eigenschaften für ein 63 ha großes Gebiet erfolgreich $\mathrm{zu}$ reproduzieren. Versuche, einzelne Bodeneigenschaften großräumig mit Hilfe von Sekundärvariablen zu schätzen, finden sich in der Literatur nur wenige (z.B. HENDERSON et al. 2005, HenGL et al. 2003, 2004). Daraus wird ersichtlich, dass einerseits noch großer Forschungsbedarf besteht, Bodeneigenschaften auch auf größeren räumlichen Skalen flächenhaft vorherzusagen, anderseits offenbart sich aber auch die unzureichende Datenlage, die der räumlichen Schätzung der drei Bodenparameter $\left(\mathrm{SOC}, \mathrm{N}_{\mathrm{t}}, \mathrm{d}_{\mathrm{b}}\right.$ ) zugrunde lag. Vor diesem Hintergrund wird die Anwendung des einfachsten räumlichen Schätzverfahrens, die Zuweisung des arithmetischen Mittelwertes, als legitime Generalisierung angesehen. Mit Blick auf die geringe Datenverfügbarkeit in tropischen Ländern sprechen STOORVOGEL \& SMALING (1998) von: „... the tropical parameter crisis.“

Die erfolgreiche Regionalisierung der Bodenarten wiederum belegt die Aussagekraft der von BÖHNER \& KÖTHE (2003) entwickelten Reliefindizes, mittels derer auch bei der im Verhältnis zur Gebietsgröße geringen Datengrundlage zuverlässige räumliche Schätzungen vorgenommen werden konnten. Im Vergleich mit der Literatur zeigt sich auch bei der Regionalisierung bodenphysikalischer Eigenschaften, dass diesbezügliche Verfahren bislang mehrheitlich auf kleineren räumlichen Skalen angewendet wurden (MCBRATNEY et al. 2003). Für weitere Untersuchungen wäre es von Interesse festzustellen, ob sich über die Abtrennung kleinerer Gebiete und der Nutzung eines höher aufgelösten DGM auch für die Parameter $\mathrm{SOC}, \mathrm{N}_{\mathrm{t}}$ und $\mathrm{d}_{\mathrm{b}}$ plausible räumliche Schätzungen durchführen lassen und ob dadurch die kleinräumigen Variabilitäten der Bodenarten in der Alluvialebene noch deutlicher nachvollzogen werden. 
Neben der Quantität der verwendeten Bodendaten ist bei der Interpretation der Ergebnisse natürlich deren Qualität von entscheidender Bedeutung. Über die möglichen, damit im Zusammenhang stehenden Unstimmigkeiten wurde in Kapitel 3.2 (Abschnitt potenzielle Fehlerquellen) berichtet und darauf soll an dieser Stelle nicht weiter eingegangen werden. Grundsätzlich jedoch sind mit den Daten räumliche und inhaltliche Unsicherheiten verbunden, die z.B. auf Messfehler oder fehlerhafte Lageangaben zurückzuführen sind. Während inhaltliche Fehler oft über Ausreißertests identifiziert werden können, lassen sich falsche Lageangaben im nachhinein kaum mehr korrigieren. Letzteres kann sich bei der Regionalisierung von Bodeneigenschaften insofern auswirken, als dass Zusammenhänge mit Schätzvariablen nicht mehr nachvollzogen werden. Künftighin wäre eine korrekte Lageangabe als Standard bei der Bodenaufnahme wünschenswert (z.B. durch GPS), um bspw. systematische Fehler, die bei der räumlichen Schätzung von Bodeneigenschaften auftreten können, zu minimieren. Als zeitaufwändig erwies sich die Zusammenstellung der Bodendaten, da diese mehrheitlich nicht publiziert wurden und als sogenannte "graue Literatur" in den Archiven von Institutionen oft nur schwer auffindbar waren. Die Vergleichbarkeit der Daten untereinander war durch die Anwendung einheitlicher Analysemethoden gewährleistet. Als arbeitsintensiv gestaltete sich die Homogenisierung der Elementgehalte, da die untersuchten Horizonttiefen oft voneinander abwichen und folglich nicht direkt vergleichbare Konzentrationen aufwiesen. Auch MURTY et al. (2002) sehen darin eine der wesentlichen Restriktionen beim Vergleich von Bodendaten aus unterschiedlichen Untersuchungen.

Das Ausmaß möglicher Veränderungen von Bodeneigenschaften unter ackerbaulicher Nutzung konnte über den Vergleich von Studien aus dem Untersuchungsgebiet mit Angaben aus der Literatur bestätigt werden. Für die Berechnung der Bodenvorräte wäre bei einigen Studien die Angabe der Lagerungsdichte hilfreich gewesen. Allgemein lässt sich über den Literaturvergleich jedoch festhalten, dass es sich bei den abgeleiteten und auf das Untersuchungsgebiet übertragenen Werte um realistische Größenordnungen handelt.

Die Zweckmäßigkeit von Landsat-Daten für die großräumige Landnutzungsanalyse muss an dieser Stelle nicht explizit diskutiert werden, da deren grundsätzliche Eignung in unzähligen Studien bestätigt wurde. Auch die Ableitung der Landnutzungssysteme gestaltete sich auf deren Grundlage und vor dem Hintergrund der Gebietskenntnis problemlos. Die gute Qualität der SRTM-Höhendaten (DGM) konnte über die Ergebnisse der Bodenartenregionalisierung bestätigt werden. Ob potenzielle Zusammenhänge zwischen Bodeneigenschaften und Relief bei kleineren Rasterweiten noch deutlicher hervortreten, müsste über ergänzende Analysen in kleineren Gebieten geprüft werden. In jedem Falle stehen mit den SRTM-Daten hervorragende Datensätze in nahezu globaler Auflösung zur Verfügung, die eine sehr gute Alternative für eine zeitaufwendige Generierung von Geländemodellen bieten. Die im Zusammenhang mit der Winderosion angewendeten Methoden zeigen, dass bereits auf der Grundlage weniger (Windgeschwindigkeit) oder, mit Blick auf die Zielsetzung, unvollständiger Datensätze (Korngrößen), allgemeine Aussagen der Bodenerosionsgefährdung 
durch Wind getroffen werden können. Eine effiziente Methode die Häufigkeit erosiver Winde allein über eine mittlere Windgeschwindigkeit $\mathrm{zu}$ bestimmen, bietet die RAYLEIGH-Verteilung. In Bezug auf die Ableitung der Korngrößenunterfraktionen lieferte der von FREDLUND et al. (2000) entwickelte Algorithmus bei allen Bodenarten gute Anpassungen an die Messwerte. Einschränkungen bestehen dahingehend, das für die Ableitung der Korngößenverteilung zumindest einge Messwerte der Unterfraktionen vorliegen müssen, an die der Algorithmus angepasst werden kann. Auf Basis der gefitteten Summenkurve konnten die prozentualen Gewichtsanteile der Feinsandfraktionen ermittelt und die relative Erodibilität der Böden im Untersuchungsgebiet bestimmt werden (K-Faktor).

Die Ausführungen lassen erkennen, dass es grundsätzlich möglich ist, qualitativ heterogene Daten unter Zuhilfenahme von Sekundärinformationen für eine großräumige Bodendegradationsanalyse so in Wert zu setzen, dass sich auf deren Grundlage plausible, quantitative Ergebnisse ableiten lassen.

\section{Ausblick, Forschungsbedarf}

Mit Blick auf die in dieser Studie aufgezeigten Entwicklungen (zunehmende Waldkonversion und Bodendegradation), stellt sich die Frage nach der weiteren Entwicklung des Agrarsektors im Department Santa Cruz. Wie anzunehmen ist, werden die verbliebenen Waldflächen der Alluvialebene in denen ein produktiver, industrieller Anbau möglich ist, mittelfristig in Kulturflächen umgewandelt werden. Da diese Entwicklung generell durch das Naturraumpotenzial limitiert ist (klimatische und pedologische Restriktionen), dürfte die Abholzung für den Ackerbau kein stetiger Prozess mehr sein. Unter Aspekten des Naturschutzes steht vielmehr zu befürchten, dass sich mit dem möglichen Aussetzen der ackerbaulichen Expansion in naher Zukunft große Viehzuchtbetriebe in den Trockenwäldern der Chiquitania im Osten Boliviens ausdehnen werden (GORRÍN 2005, IBISCH et al. 2002).

Im Zusammenhang mit den Ergebnissen dieser Studie, wären im Department Santa Cruz folgende Aspekte für weiterführende Untersuchungen von Interesse:

- Aus ökonomischer Sicht sollten Modellrechnungen durchgeführt werden, welche Kosten und welchen Nutzen landwirtschaftliche Betriebe bei der Umstellung von konventioneller zu konservierender Bodenbearbeitung zu erwarten hätten.

- Aus der Expansionszone existieren so gut wie keine Untersuchungen, die sich mit möglichen Veränderungen von Bodeneigenschaften unter landwirtschaftlicher Nutzung beschäftigen. Hier wären entsprechende Studien dringend notwendig. Das gleiche gilt im gesamten Department für Untersuchungen, die die potenziellen Veränderungen chemischer und physikalischer Bodeneigenschaften unter Weidenutzung (mit und ohne Weidemanage- 
ment) analysieren. In diesem Zusammenhang wäre auch wichtig, die Expansion der Viehzucht- bzw. Viehhaltungsbetriebe der letzten zwei Dekaden zu untersuchen und denkbare zukünftige Entwicklungen abzuleiten.

- Auf den aufgelassenen, degradierten Anbauflächen sollte untersucht werden, wie hoch die natürliche Anreicherung organischer Substanz ist und ob, bspw. durch die Kultivierung produktiver Gräser, Nährelementanreicherungen in der Höhe zu erwarten wären, die mittelfristig eine zumindest bodenschonende Nutzung zuließen.

- Von pedologischer Seite wären überdies Untersuchungen interessant, die den Einfluss der Bodenbearbeitung auf eine mögliche Veränderung im Korngrößenspektrum der Böden analysieren.

- Die bereits angedeuteten klimatischen Veränderungen, die mit großflächigen Rodungen und einer anschließenden landwirtschaftlichen Nutzung einher gehen, sollten über weitere Studien verfolgt werden.

- Unter sozioökonomischen Aspekten wären Studien nützlich die der Frage nachgehen, inwieweit die zunehmende Bodendegradation im Department Auslöser verstärkter Migrationsströme ist.

- Letztlich stellt sich die Frage, inwieweit die im staatlichen Landnutzungsplan (PLUS) von 1996 formulierten gesetzlichen Vorgaben im Department tatsächlich eingehalten wurden.

Das Ausmaß der aktuellen Umweltveränderungen im Department Santa Cruz verdeutlicht, dass dringender Handlungsbedarf besteht die Landwirtschaft in dieser Region nachhaltiger zu gestalten, um den aus ökonomischen Zwängen heraus resultierenden Nutzungsdruck auf zusätzliche Flächen zu reduzieren. Da ein großer Teil der Agrarflächen erst eine junge Nutzungsgeschichte aufweist, bestünde die Chance, der fortschreitenden Bodendegradation über standortgerechte Bewirtschaftungsweisen entgegenzuwirken. Ob allerdings vor dem Hintergrund der staatlichen Entwicklungsplanungen deren Einführung von großer sozialer und politischer Akzeptanz sein wird, bleibt zweifelhaft. 


\section{ZUSAMMENFASSUNG}

Die vorliegende Arbeit gibt die Ergebnisse eines von der Deutschen Forschungsgemeinschaft (DFG) geförderten Projektes zur Analyse der Waldkonversion und Bodendegradation im tropischen Tiefland von Ostbolivien wieder. Das Ziel der Untersuchung war, auf der Grundlage existierender Bodenuntersuchungen sowie allgemein verfügbarer Daten und Angaben aus der Literatur, die maßgeblichen durch ackerbauliche Nutzung ausgelösten Prozesse der Bodendegradation (Verlust organischer Bodensubstanz, Bodenverdichtungen) in diesem Raum zu quantifizieren und regional darzustellen. Zudem wurde eine allgemeine Abschätzung der Bodenerosionsgefährdung durch Wind vorgenommen. Die Studie hatte den Zweck, eine Methode aufzuzeigen, mit der sich vorhandene Daten in Ländern mit einer geringen Datenverfügbarkeit für eine regionale, quantitative Analyse in Wert setzten lassen.

Methodisch wurde anhand von Satellitendaten (Landsat) die Landnutzungsentwicklung der letzten zwei Jahrzehnte (1984-2001) ermittelt und darüber den Anbauflächen ein Nutzungszeitraum zugewiesen. Auf derselben Grundlage wurden die Landnutzungssysteme des Untersuchungsraums regional klassifiziert (Kleinbauern, industrielle Landwirtschaft). Die im Zusammenhang mit den Degradationsprozessen wesentlichen Bodeneigenschaften (Bodenart, organische Bodensubstanz, Lagerungsdichte) wurden insgesamt 370 Profilbeschreibungen des Untersuchungsgebietes entnommen und homogenisiert. Auf Grundlage eines digitalen Geländemodells (DGM) wurden Flächendatensätze des Reliefs berechnet sowie Klimaparameter unter Nutzung von Daten eines allgemeinen Zirkulationsmodells (GCM) und lokaler Klimastationsdaten räumlich generiert. Durch schrittweise multiple Regression wurden räumliche Abhängigkeiten der punktuellen Bodeninformationen unter natürlicher Vegetation (Ausgangsniveau) zu Klima- und Reliefparametern überprüft und, im Falle eines Zusammenhangs, die entsprechende Bodeneigenschaft über die ermittelte Regressionsfunktion in die Fläche übertragen. Die zeitliche Größenordnung kultivierungsbedingter Änderungen von organischer Bodensubstanz (SOM) (in der Studie anhand der Parameter organischer Kohlenstoff [SOC] und Gesamtstickstoff $\left[\mathrm{N}_{\mathrm{t}}\right]$ untersucht) und Lagerungsdichte $\left(d_{b}\right)$ wurden aus der Literatur und lokalen Untersuchungen abgeleitet und unterschiedliche Entwicklungen zwischen den Landnutzungssystemen herausgearbeitet. Die Werte wurden mit dem jeweiligen Ausgangsniveau beider Bodenparameter und der Entwicklung der Anbauflächen in Beziehung gesetzt und deren aktueller Status abgeschätzt. Das potenzielle Winderosionsrisiko wurde über Erodibilität der Böden und die Erosivität der Winde bestimmt. Die Bodenerodierbarkeit wurde aus der Bodenart und dem SOM-Gehalt über die Ableitung eines K-Faktors ermittelt und die Häufigkeit erosiver Winde aus Monatmittelwerten der Windgeschwindigkeit unter Zuhilfenahme der RAYLEIGH-Verteilung regional spezifiziert.

Die räumliche Varianz der Bodenarten konnte über Reliefparameter erklärt $\left(\mathrm{r}^{2}=40-50 \%\right)$ und auf die Fläche übertragen werden. Für die Parameter SOC, $\mathrm{N}_{t}$ und $\mathrm{d}_{\mathrm{b}}$ war eine plausible 
räumliche Schätzung nicht möglich. Für diese wurden aus den vorhandenen Profilbeschreibungen allgemeine, bodenartenabhängige Mittelwerte abgeleitet und als Ausgangsniveau angenommen.

Die Ergebnisse der Landnutzungsanalyse belegen die außerordentliche Entwicklungsdynamik, die im ostbolivianischen Tiefland in den letzten zwei Jahrzehnten zu beobachten ist. In diesem Zeitraum wurden ca. $13.000 \mathrm{~km}^{2}$ Primärwald gerodet und fast ausschließlich in eine ackerbauliche Nutzung überführt. Diese Entwicklung ist mehrheitlich Folge des südamerikanischen Soja-Booms, der speziell in den 1990er Jahren zu einer erheblichen Expansion der industriellen Landwirtschaft im Department Santa Cruz geführt hat. Allein auf den Zeitraum von 1992-2001 entfallen gut vier Fünftel der seit 1984 festgestellten Rodungen. Die höchsten Abholzungsraten finden sich im nördlichen Untersuchungsraum, da die Niederschlagsverhältnisse für einen ertragreichen Anbau günstiger sind und z.T. zwei Anbauzyklen pro Jahr erlauben. Die Abholzungsraten in diesem Bereich zählten in den 1990er Jahren weltweit mit zu den höchsten. Mit zunehmender Breitenlage steigt das hydrologische Defizit und mithin das Ertragsrisiko, demzufolge die Abholzungsraten Richtung Chaco deutlich geringer sind. In zeitlicher Hinsicht wurde knapp die Hälfte der Anbauflächen 2001 nicht länger als neun Jahre genutzt. Der größte Teil davon liegt in der jüngeren Landwirtschaftszone, der Expansionszone. Die ältesten Anbauflächen befinden sich in der ersten landwirtschaftlichen Entwicklungszone (zentrale Zone). Von diesen sind schon annähernd $700 \mathrm{~km}^{2}$ wegen der fortschreitenden Bodendegradation nicht mehr rentabel zu bewirtschaften und werden allenfalls noch als extensives Weideland genutzt.

Bei den Böden der zentralen Zone handelt es sich mehrheitlich um sandige Lehme, die aufgrund ihrer geringen SOM-Gehalte im Vergleich mit den tonreicheren Lehmen der Expansionszone schneller ihre natürliche Produktivität verlieren. Indes liegen die relativen Verluste, unabhängig von der Bodenart, auf einem vergleichbaren Niveau. Allein während der ersten zehn Anbaujahre ist die organische Substanz in feinkörnigen Böden aufgrund der im Vergleich mit sandigen Böden höheren Aggregierung besser vor Zersetzung geschützt. Als ursächlicher Prozess des fortschreitenden Produktivitätsverlustes der Böden wurden konventionelle Bodenbearbeitungspraktiken (regelmäßiger Pflugeinsatz) identifiziert, infolge derer sich das Ausgangsniveau der organischen Substanz im Oberboden innerhalb von nur zwei Dekaden durchschnittlich um die Hälfte verringert. Die sandigen Lehme gelten zu diesem Zeitpunkt mit SOM-Vorräten von im Mittel unter $30 \mathrm{t} \mathrm{ha}^{-1}(0-20 \mathrm{~cm})$ als humusarm, demgegenüber die feinkörnigen Lehme bei gleicher Nutzungsdauer aufgrund ihres höheren Ausgangsniveaus noch besser mit Nährstoffen versorgt sind (ca. $40 \mathrm{t} \mathrm{ha}^{-1}$ ). Dennoch konnte gezeigt werden, dass auch auf diesen Böden die Produktivität infolge der hohen relativen Verluste bei Fortdauer der derzeitigen Bewirtschaftungsweisen mittelfristig zurückgeht.

Die Analyse der Bodenverdichtung dokumentiert, dass in Abhängigkeit von der mechanischen Beanspruchung bei allen Bodenarten des Untersuchungsgebietes Verdichtungen auftreten können. Die relative Zunahme der Lagerungsdichte beträgt nach langjähriger Bewirt- 
schaftung im Mittel zwischen 14-43\%. Folglich ist auf allen mechanisch kultivierten Anbauflächen im Untersuchungsgebiet z.T. mit Verdichtungen im Oberboden zu rechnen, die das Wurzel- und somit auch das Pflanzenwachstum limitieren. Einzig im kleinbäuerlichen slash and burn System konnten keine negativen Veränderungen der bodenphysikalischen Eigenschaften festgestellt werden. Das Risiko von Verdichtungen steigt mit zunehmenden Feinsubstratanteil im Boden, da die Zeit, in der sich ein Boden in einem für die Bearbeitung optimalen Feuchtebereich befindet, kleiner wird. Im Untersuchungsgebiet bedeutet das speziell für die feinkörnigen Lehme im humideren Norden und Nordwesten ein erhöhtes Risiko, da dort die Bearbeitungsintensität und somit die mechanische Beanspruchung der Böden am höchsten ist (2 Anbauzyklen pro Jahr). In Bezug auf das Landnutzungssystem steigt das Risiko mit zunehmender Schlaggröße und geringer Diversifikation der Anbaukulturen. Daraus leitet sich eine hohe Gefährdung für die Anbauflächen der Agroindustrie ab, während diese in den Gebieten der Kleinbauern mit mechanisierter Bewirtschaftung geringer eingeschätzt wird.

Winderosion ist vorwiegend ein Problem auf den sandigen Lehmen der zentralen Zone, kann aber durch die anhaltende Zerstörung von Bodenaggregaten infolge von Bearbeitungsprozessen auch zu einem Problem auf den Anbauflächen in der Expansionszone werden. Allerdings ist das Risiko von Bodenabträgen in dieser Zone insgesamt geringer, da im Vergleich mit der zentralen Zone die mittlere Windgeschwindigkeit zurückgeht und, zumindest in den Gebieten der Agroindustrie, mächtige Windschutzstreifen als Erosionsschutz installiert wurden. Der Anteil potenziell erosiver Winde liegt in der zentralen Zone im Jahresverlauf bei $14 \%$ und steigt in der Trockenzeit auf $21 \%$. Im Norden und Osten sind die Windverhältnisse moderater. Der Anteil erosiver Windgeschwindigkeiten liegt dort zwischen 1-4 \% pro Jahr, in der Trockenzeit bei maximal $8 \%$. Aus der Expansionszone liegen keine längerfristigen Windaufzeichnungen vor, folglich werden sich die mittleren Windgeschwindigkeiten zwischen den Werten der zentralen Zone und denen aus dem Norden und Osten bewegen. In klimatischer Hinsicht steigt das Winderosionsrisiko zudem mit zunehmender Trockenheit, weshalb die Wahrscheinlichkeit potenzieller Erosionsschäden durch Wind im semi-ariden Süden am höchsten ist. Mit Blick auf die pedologischen Eigenschaften sind Böden mit Feinsandgehalten von über 60 Gew.-\% und Aggregatanteilen von unter Gew.-15 \% am leichtesten vom Wind erodierbar. Gleichzeitig steigt das Risiko mit abnehmenden SOMGehalten. Bei Feinsandgehalten von weniger als 30 Gew.-\% und einem Anteil der Aggregate von über 50 Gew.-\% sind die Böden kaum noch vom Wind erodierbar. Bezogen auf das Landnutzungssystem steigt das Winderosionsrisiko mit zunehmender Schlaggröße und der Häufigkeit der Bodenbearbeitung. Demnach ist die Wahrscheinlichkeit von Bodenverlusten in den Gebieten der industriellen Landwirtschaft, insbesondere in den Mennonitenkolonien wegen der oft unzureichenden Windschutzbepflanzungen, am höchsten.

Die Ergebnisse der Studie zeigen, dass die regelmäßige Pflugbewirtschaftung in tropischen Breiten zu einem raschen Verlust der natürlichen Bodenproduktivität führt. In diesem 
Zusammenhang muss, aufgrund der positiven chemischen (Nährstoffspeicher) und physikalischen (Aggregatstabilität) Bodeneigenschaften, besonders der schnelle Abbau der organischen Substanz im Boden als kritisch bewertet werden. Als Alternative sollte ein Wechsel von konventioneller zu konservierender Bodenbearbeitung, die in der industriellen Landwirtschaft anderer tropischer und subtropischer Länder schon länger erfolgreich betrieben wird, auch in Ostbolivien in Betracht gezogen werden. Nur eine bodenschonende Bearbeitung kann die natürliche Fruchtbarkeit der Böden in diesen Breiten langfristig erhalten und damit auch ihre Funktion als Produktionsstandort sichern.

Die angewendete Methodik ist auf andere Gebiete übertragbar, sofern die dortigen Rahmenbedingungen (z.B. Bewirtschaftungsweise) bekannt sind. Durch die ergänzende Nutzung allgemein verfügbarer Flächendaten sowie dem Einbezug von Literaturangaben, die sowohl als auch erfahrungsgemäß problemlos über das Internet zu beziehen sind, lassen sich vorhandene Bodendaten für eine quantitative, räumliche Analyse in Wert setzten. Als zeitaufwendig gestaltet sich vielmehr das Auffinden ,,lokaler" Daten, da diese in der Regel nicht veröffentlicht wurden und auch in örtlichen Bibliotheken nicht unbedingt zugänglich sind. 


\section{AbSTract}

The present work was carried out in the context of a research project entitled 'Forest conversion and soil degradation in the tropical lowlands of Eastern-Bolivia' financed by the German Research Society (DFG). The objective of the study was to assess the main types of soil degradation such as nutrient decline (e.g. soil organic matter) and soil compaction caused by different land use systems, as well as the susceptibility of the soils to wind erosion on a regional scale. Information about soils was derived from existing soil studies. The purpose was, to develop a method for the application of a quantitative approach based on existing data in countries with low data availability.

To obtain information about current and former land uses (period: 1984-2001) and the applied cultivation system (e.g. small holder, industrial farming) several Landsat images were analyzed. Based on the results of the change detection analysis, different land use periods were assigned to the cultivation areas. The main soil properties (soil texture, soil organic matter, bulk density) were derived and homogenized from 370 local profile descriptions. A digital terrain analysis has provided continuous primary and secondary terrain attributes. Climate variables were spatially generated with a General Circulation Model (GCM) statistical downscaling approach, which is based on empirical transfer functions that describe the observed local climate. The calculated climate and terrain attributes were used as predictor variables for continuous regionalization of soil properties. Spatial prediction functions were defined by means of stepwise multiple regression analyses. Relative changes in soil properties (soil organic carbon, total nitrogen and bulk density) following cultivation of previously untilled soils, were derived using data from a literature review and local soil studies. To assess the current state of specific soil properties, the rates of change were related to the respective initial level. The potential risk of wind erosion was assessed by the relative soil erodibility and the wind erosivity. The erodibility of soils was determined as a function of soil texture and organic matter content (expressed as K-factor), whereas the frequency of erosive wind speeds was estimated from monthly mean values using the RAYLEIGH function.

As far as soil texture is concerned $40-50 \%$ of the variance were explained by terrain parameters and could be spatially predicted. Reasonable spatial prediction functions regarding soil organic carbon, total nitrogen and bulk density were not found, hence general mean values related to soil texture were determined from the profile descriptions and assumed as initial levels.

The results of the land use analysis highlights the intense development which has taken place in the Eastern Bolivian lowlands during the last two decades. Within the study area, approximately $13.000 \mathrm{~km}^{2}$ forest were converted to agricultural use between 1984 and 2001 . This was mainly attributed to the soy boom in South America, which led to a rapid expansion of industrial soy-bean farms in the Santa Cruz department, especially during the 1990s. About four-fifths of the total deforestation account solely for the period off 1992 to 2001 . Highest 
deforestation rates occurred in the northern sector of the study area due to more favorable climatic conditions, which enable the cultivation of two crops per year. Here, the estimated deforestation rates during the 1990s were among the highest in the world. Clearing activities are decreasing significantly with increasing latitude, due to a growing hydrological deficit and thus diminishing yields and an increasing cropping risk. About half of the total cropland had not been used for longer than nine years in 2001. Most of these areas are situated in the new agricultural 'expansion zone' east of the Río Grande. The older cropping areas are located closer to the city of Santa Cruz, in the so-called 'central zone'. Due to ongoing soil degradation processes, approximately $700 \mathrm{~km}^{2}$ of land in this sector are currently not profitable for cultivation anymore and hence mostly turned into extensive pasture land.

Sandy loams predominate in the central zone. In contrast to more fine-textured soils of the expansion zone (silty and clayey loams), they are characterized by a more rapid loss of fertility due to lower initial soil organic matter contents. However, the relative losses found for all soil types are comparable in the long term. Solely within the first ten years of cropping the more fine-textured soils exhibit slower decomposition rates due to higher aggregation and thus better physical protection of the soil organic matter. Conventional tillage (plow-based systems) has been identified as the principal factor responsible of triggering soil degradation in the Santa Cruz region. This technique led to an average loss of about $50 \%$ of organic matter in the topsoil within only two decades of continuous cropping. After long-term cultivation the mean soil organic matter stock of sandy loams was less than $30 \mathrm{tha}^{-1}(0-20$ $\mathrm{cm}$ ) and can be considered as low, whereas the fine-textured loams were still better supplied with plant nutrients (about $40 \mathrm{t} \mathrm{ha}^{-1}$ ), due to their higher initial amount of soil organic matter. However, it has been shown that these soils also lost large amounts of soil organic matter due to the applied cultivation techniques. This will lead to a drastic decline of soil productivity in the medium-term.

The analysis of soil compaction in the study area has shown, that under mechanized cultivation severe compaction with a negative impact on plant growth and yields can occur in all soil types. The mean relative increase of topsoil bulk density was between 14 and $43 \%$. No negative changes in physical soil properties were observed for the traditional slash and burn system. The susceptibility to compaction increases with an increasing proportion of fine soil particles, because of the reduced number of optimal working days depending on the soil moisture content. Thus, the fine-textured soils in the more humid areas in the north and northwest face a high risk of compaction, due to a high mechanical impact as a consequence of double cropping. With reference to the land use system, the susceptibility to compaction increases with increasing field size and decreasing crop diversity. Hence, soils in the highly mechanized industrial cropping areas generally face a higher risk to compaction, compared to smallholder areas with mechanized production.

Wind erosion predominately is a problem of the sandy loams of the central zone, but can also occur in the cropping areas of the expansion zone, due to the destruction of soil 
aggregates by plowing. However, the susceptibility to wind erosion in this sector is generally lower, because of reduced wind speed and huge wind breaks in the areas of agro-industrial farms. About $14 \%$ of the winds in the central zone are highly erosive, with a proportion of 21 $\%$ during the dry season. In contrast, the mean proportion of erosive wind speeds in the northern and eastern part of the study area only accounts for $1-4 \%$, with a maximum of $8 \%$ during the dry season. There are no long-term wind measurements for the expansion zone, therefore the mean annual wind speeds are presumed to lie between those of the central zone and those of the north and the east. Climatologically, the wind erosion risk increases with increasing aridity, thus the probability of potential erosion damages is highest in the semi-arid sector of the study area. Regarding the textural properties, soils that contain $60 \%$ or more fine sand and a proportion of aggregates less than $15 \%$ are most susceptible to wind erosion. Additionally, the risk increases with decreasing contents of soil organic matter. In contrast, soils with a content of fine sand below $30 \%$ and a proportion of aggregates larger than $50 \%$ are hardly liable to wind erosion. Regarding the land use system the potential wind erosion risk increases with increasing field-size and the frequency of tillage. Thus, the areas of industrial agriculture are most liable to wind erosion, especially in the colonies of Mennonite farmers, where an insufficient installation of wind breaks is found.

The results of the study show, that conventional farming practices under tropical and subtropical conditions lead to a rapid loss of soil fertility. In this context the rapid loss of soil organic matter is of particular importance, because of its positive function in the maintenance as well as improvement of many chemical (e.g. nutrient pool) and physical (e.g. aggregate stability) soil properties. A possible alternative to maintain soil organic matter pools in tropical agriculture consists of the conversion from conventional to conservation tillage management systems, which have already been successfully applied in other tropical or subtropical countries for a long time. These techniques should be taken into account for future agricultural practices in Eastern-Bolivia. Only soil conservation practices maintain long-term soil fertility and thus productivity in areas with warm climates.

The study's methodology can be applied to other areas, if the general local conditions are known (e.g. cultivation systems). By the additional use of secondary data (continuous spatial data) and topic-specific information (literature review), which are normally easy to obtain through the internet, spatially quantitative analysis of soil related questions can be performed based on existing local soil data. The search for measured local data normally turns out to be time-consuming, due to the fact that these are often neither published nor accessible in local libraries. 


\section{RESUMEN}

El presente trabajo fue ejecutado en el contexto del proyecto "Conversión de Bosques y degradación de suelos en las tierras bajas del oriente Boliviano", finaciado por la Sociedad Alemana de Investigación (DFG). El objetivo del estudio fue la estimación de los principales tipos de degradación de suelos, como la disminución de nutrientes (p. ej. materia orgánica) y la compactación, causadas por los diferentes sistemas de uso del suelo, así como la suceptibilidad a erosión a escala regional. La información sobre suelos fue derivada de estudios preexistentes. El propósito fue el desarrollo de un método de aplicación de un enfoque cuantitativo basado en datos existentes de países con baja disponibilidad de datos.

Para la obtención de información sobre el uso del suelo actual y anterior (periodo: 19842001) y el sistema de cultivo aplicado (p. ej. agricultura a pequeña escala, agricultura industrial), una serie de imágenes Landsat fueron analizadas. En base a los resultados del análisis temporal de cambios, diferentes períodos de usos del suelo fueron asignados a las áreas de cultivo. Las principales propiedades del suelo (textura, materia orgánica, nivel de compactación) fueron derivadas y homogeneizadas de 370 descripciones de pérfiles locales. Los atributos continuos del terreno, de carácter primario y secundario, fueron obtenidos de un análisis digital del terreno. Variables climáticas espaciales fueron generadas con un Modelo de Circulación General (MCG), usando un downscaling estadístico basado en la transferencia empírica de funciones que describen el clima local observado. Los atributos del terreno y del clima calculados fueron usados como variables predictivas para la regionalización continúa de las propiedades del suelo. Las funciones de predicción espacial fueron definidas por medio de un análisis de regresión múltiple por etapas.

Cambios relativos en las propiedades del suelo (carbón orgánico, nitrógeno total y nivel de compactación) fueron generados usando datos provenientes de literatura científica y estudios locales del suelo. Para estimar el estado actual de las propiedades específicas del suelo, las tasas de cambio fueron relacionadas al nivel inicial correspondiente. El riesgo potencial de erosión fue evaluado por la erodabilidad relativa del suelo y la erosividad del viento. La erodabilidad del suelo fue determinada como una función de la textura y el contenido de materia orgánica (factor k), mientras que la frecuencia de vientos de velocidades erosivas fué estimada por valores promedios mensuales usando la función de RAYLEIGH.

La variación espacial de los tipos de suelo se explicó y fue regionalizada mediante el análisis de los parámetros de relieve $\left(\mathrm{r}^{2}=40-50 \%\right)$. Para los parámetros carbón orgánico, nitrógeno total y nivel de compactación no pudo realizarse una regionalización espacial plausible, por lo cual valores promedio para las texturas de suelo fueron determinados en base a descripciones existentes de perfiles, valores que se tomaron como valores iniciales.

Los resultados del análisis de uso del suelo muestran la dimensión de crecimiento al que ha estado sometido el oriente Boliviano en las últimas dos décadas. En el área de estudio, unos $13000 \mathrm{~km}^{2}$ de bosques primarios han sido incorporados al uso agrícola en el período 1984- 
2001. Éste proceso se debió principalmente al auge sudamericano de la soya en los años 90, durante la cual se expandió la agricultura mecanizada en el Departamento de Santa Cruz. Un aproximado de cuatro quintos de la deforestación total ocurrió en el período 1992-2001. Las mayores tasas de deforestación ocurrieron en el norte del área de estudio, debido principalmente a las condiciones climáticas favorables que, en parte, permiten dos períodos de cultivos al año. La tasa de deforestación de la zona estuvo entre las más altas del mundo en la década de 1990. Con el incremento de la latitud, se identifica una disminución significativa de los desmontes, debido al déficit hídrico y la consecuente disminución de la productividad de las cosechas y el aumento del riesgo para los cultivos.

Considerando el factor tiempo, casi la mitad de la superficie agrícola total del 2001 no fue cultivada por un período mayor de nueve años. La mayoría de esas áreas están situadas en la nueva área de expansión agrícola al este del Río Grande. Los cultivos más antiguos están ubicados en las cercanías de la ciudad de Santa Cruz, en la primera zona de crecimiento agrícola denominada "zona central". En ese sector, debido a procesos avanzados de degradación del suelo, unos $700 \mathrm{~km}^{2}$ de terreno han perdido el nivel de rentabilidad para cultivos y en su mayoría son utilizados para el pastoreo extensivo de ganado.

Suelos arenosos dominan en la zona central. En contraste, en la zona de expansión agrícola se encuentran suelos de texturas más finas (limosos y arcillosos), que al tener un contenido inicial menor de materia orgánica pierden su fertilidad natural más rápidamente. Sin embargo, la pérdida relativa de fertilidad ocurre de igual manera para todos los tipos de suelo. Solamente en los diez primeros años de cultivo, los suelos de textura más fina muestran tasas más lentas de descomposición (en comparación con suelos arenosos) debido al alto nivel de agregación, lo que actúa como una protección física para la materia orgánica del suelo. El arado convencional (frecuente) ha sido identificado como el principal factor responsable de desencadenar la degradación del suelo en la región de Santa Cruz, por lo cual, solo en dos décadas la materia orgánica del suelo superficial ha disminuido a la mitad. Luego de un largo período de cultivo, la cantidad de materia orgánica almacenada en los suelos franco-arenosos era menor a $30 \mathrm{t} \mathrm{ha}^{-1}(0-20 \mathrm{~cm})$, por lo que se pueden definir como suelos pobres en humus. Los suelos franco-limosos, sujetos al mismo período de cultivo, estaban mejor abastecidos de nutrientes orgánicos; ya que presentaban inicialmente mayor contenido de materia orgánica (alrededor de $40 \mathrm{t} \mathrm{ha}^{-1}$ ). Sin embargo, se observa que estos suelos también pierden grandes cantidades de materia orgánica debido a las técnicas de cultivo aplicadas, lo que origina a mediano plazo un drástico declive de la productividad.

El análisis del nivel de compactación del suelo mostró una relación entre la siembra mecanizada y un crecimiento negativo de las plantas y el nivel de productividad y también, que la compactación puede ocurrir en todos los tipos de suelo. El incremento promedio relativo de compactación de la capa superficial del suelo estuvo entre el 14 y 43 \%. Ningún cambio negativo en las propiedades físicas de los suelos fue observado solamente en el sistema tradicional de tala y quema. La susceptibilidad de compactación aumenta con un 
incremento de la proporción de partículas finas del suelo superficial, debido al número reducido de días óptimos de labor que dependen del contenido de humedad del suelo. De ésta manera, los suelos de texturas finas en las áreas más húmedas en el norte y al noroeste enfrentan un mayor riesgo de compactación, debido al mayor impacto mecánico como consecuencia de la siembra de dos cultivos al año. En relación al sistema de uso del suelo, la susceptibilidad de compactación incrementa con en aumento del tamaño de la parcela y la disminución de la diversidad de cultivos. Por lo que suelos ubicados en áreas con alta mecanización industrial presentan un mayor riesgo de compactación, en comparación con aquellos ubicados en pequeñas granjas mecanizadas.

La erosión eólica es un problema predominante de los suelos franco-arenosos de la zona central, pero también puede encontrarse en los cultivos de la zona de expansión, debido a la destrucción de agregados del suelo por el arado. No obstante, la susceptibilidad a la erosión eólica en éste sector es relativamente menor, debido a la velocidad reducida del viento y a los rompevientos de gran tamaño colocados en las zonas agroindustriales. Alrededor del $14 \%$ de los vientos en la zona central son altamente erosivos, con una proporción que asciende al 21 $\%$ en la estación seca. Contrariamente en la parte norte y este del área de estudio, la proporción promedio de velocidades erosivas solo alcanza un rango del 1-4 \%, con un máximo de $8 \%$ en la estación seca. La zona de expansión carece de largos registros de vientos, por lo que se asume que las velocidades anuales se encuentran entre las de la central zona y las del norte y este. Climatológicamente hablando, el riesgo de erosión eólica se incrementa con la aridez, por lo que el sector semi-árido ubicado al sur del área de estudio presenta la mayor probabilidad de daños potenciales por erosión. En relación a la textura, los suelos más susceptibles a la erosión eólica son aquellos que contienen $60 \%$ o más de arena de grano fino y una proporción de agregados menor al $15 \%$. Adicionalmente, éste riesgo aumenta al disminuir el contenido de materia orgánica del suelo. Suelos con un contenido de arenas finas por debajo del $30 \%$ y una proporción de agregados mayor al $50 \%$ son muy poco susceptibles a la erosión eólica. Considerando el sistema de uso del suelo, el riesgo potencial de erosión eólica aumenta positivamente con el tamaño de la parcela y la frecuencia de labranza. De ésta manera las áreas con agricultura industrializada son más propensas a éste tipo de erosión, especialmente las colonias Menonitas, donde los rompevientos existentes son insuficientes.

Los resultados del estudio muestran que las prácticas agrícolas convencionales bajo condiciones tropicales y sub-tropicales generan una pérdida acelerada de la fertilidad del suelo. En este contexto la pérdida rápida de materia orgánica del suelo tiene importancia significativa debido a su función de mantenimiento y mejora de muchas propiedades químicas (p. ej. almacenaje de nutrientes) y físicas (p. ej. estabilidad de los agregados) de los suelos. Una posible alternativa para mantener el contenido de materia orgánica de los suelos agrícolas tropicales consiste en el cambio de sistemas de manejo convencionales a sistemas de labranza conservacionista, que desde hace tiempo se vienen aplicando satisfactoriamente en otros 
países tropicales y sub-tropicales. Éstas técnicas deben tomarse en consideración para futuras prácticas agrícolas en el oriente Boliviano. A largo plazo, solo las prácticas de conservación de suelos pueden mantener su fertilidad y productividad en áreas tropicales.

La metodología aplicada es transferible a otras áreas, si las condiciones previas locales de cultivo (p. ej. sistemas de cultivo) son conocidas. Mediante el uso adicional de datos secundarios (datos continuos espaciales) e información temática específica (literatura científica), de fácil acceso por Internet, se pueden realizar análisis espaciales cuantitativos de suelos, apoyados en información preexistente. La búsqueda de datos locales requiere por lo general inversión muy costosa de tiempo, debido a que normalmente estos datos son inéditos o no se dispone del libre acceso a bibliotecas locales. 


\section{LITERATURVERZEICHNIS}

Abu-Hamdeh, N.H. (2003): Compaction and Subsoiling Effects on Corn Growth and Soil Bulk Density. - Soil Science Society of America Journal 67 : 1213-1219

ABUBAKAR, S.M. (1997): Monitoring land degradation in the semiarid tropics using an inferential approach: The Kabomo basin case study, Nigeria. - Land Degradation and Development $8: 311-323$

Achard, F., H.D. Eva, H.-J. Stibig, P. Mayaux, J. Gallego, T. Richards \& J.-P. MALINGREAU (2002): Determination of deforestation rates of the world's humid tropical forests. - Science 297 : 999-1002

AG BoDEN (1994): Bodenkundliche Kartieranleitung. - 4. Auflage, 392 pp., Hannover

AgRAR- \& HYDROTECHNIK (1973): Proyecto de desarollo agroindustrial Abapo-Izozog. Pedologia, Estudio detallado de suelos de la Granja-Piloto. - Inf. Tec. 2/1973, Essen

AgRAR- \& HYdRoteChNiK (1974a): Proyecto de desarollo agroindustrial Abapo-Izozog. Estudio detallado de suelos de las tres sub-estaciones. - Inf. Tec. 7/1974, Essen

AgRAR- \& HYDROTECHNIK (1974b): Proyecto de desarollo agroindustrial Abapo-Izozog. Pedologia, Estudio semi-detallado de suelos. - Inf. Tec. 9/1974, Essen

AL-KaISI, M.M., X. YIN \& M.A. LichT (2005): Soil carbon changes as influenced by tillage and cropping systems in some Iowa soils. - Agriculture, Ecosystems and Environment 105 : 635-647

ANIKwe, M.A.N., M.E. OBI \& N.N. AgBIM (2003): Effect of crop and soil management practices on soil compatibility in maize and groundnut plots in a Paleustult in Southeastern Nigeria. - Plant and Soil $253:$ 457-465

Araujo, M.A., C. A. Tormenta \& A. P. Silva (2004): Propriedades físicas de um Latossolo Vermelho distrófico cultivado e sob mata nativa. - Revista Brasileira de Ciência do Solo 28 (2) : $337-345$

Arevalo, L.A., J.C. Alegre, D.E. BAndy \& L.T. SzOTt (1998): The effect of cattle grazing om physical and chemical properties in a silvopastoral system in the Peruvian Amazon. Agroforestry Systems 40 : 109-124

Asner, G.P., A.R. Townsend, M.M.C. Bustamante, G.F.B. Nardoto \& L.P. Olander (2004): Pasture degradation in the central Amazon: linking changes in carbon and nutrient cycling with remote sensing. - Global Change Biology 10 : 844-862

Assouline, S. (2002): Modeling soil compaction under uniaxial compression. - Soil Science Society of America Journal $66: 1784-1787$

BAGNOLD, R.A. (1941): The physics of blown sand and desert dunes. - Chapman and Hall Ltd., London

BARBER, R.G. (1994a): Persistence of loosened horizons and soybean yield increases in Bolivia. - Soil Science Society America Journal 58 : 943-950

BARBER, R.G. (1994b): Influencia del aluminio de subsuelo en los rendimientos de cultivos con fertilización de N y P. - Avances de Investigación 10, CIAT/MBAT, Santa Cruz, Bolivia

BARBER, R.G. (1995): Soil degradation in the tropical lowlands of Santa Cruz, Eastern Bolivia. - Land Degradation and Rehabilitation 6 : 95-107 
BArber, R.G., C. Herrera \& O. DiAz (1989): Compaction status and compaction susceptibility of alluvial soils in Santa Cruz, Bolivia. - Soil Tillage and Research 15 : 153167

Barber, R.G., F. Navarro, M. Orellana, E. Rojas \& D. Romero (1992): Diagnóstico de los problemas de suelos para agricultores mecanizados en las áreas: Central, Las Brechas y Pailon-Los Troncos del departamento de Santa Cruz y las actividades a ser realizadas por la Sección de Suelos del CIAT, 1991-2000 adelante. - CIAT/MBAT, Santa Cruz, Bolivia

BARBER, R.G. \& D. ROMERO (1994): Effects of Bulldozer and chain clearing on soil properties and crop yields. - Soil Science Society of America Journal 58 : 1768-1775

BARBER, R.G. \& F. NAVARRO (1994a): Evaluation of the soil characteristics of 14 cover crops used in a soil rehabilitation trial. - Land Degradation and Rehabilitation 5 : 201-214

BARBER, R.G. \& F. NAVARRO (1994b): The rehabilitation of degraded soils in eastern Bolivia by sub-soiling and the incorporation of cover crops. - Land Degradation and Rehabilitation $5: 247-259$

BARBER, R.G. \& O. DÍAZ (1994): Maintenance of yields and soil fertility in non-mechanized cropping systems, Bolivia. - Soil Science Society of America Journal 58 : 858-866

Barber, R.G., M. Orellana, F. Navarro, O. Díaz \& M.A. Soruco (1996): Effects of conservation and conventional tillage systems after land clearing on soil properties and crop yield in Santa Cruz, Bolivia. - Soil \& Tillage Research 38 : 133-152

BARBIER, E. (1997): The economic determinants of land degradation in developing countries. - Philosophical Transactions of The Royal Society B 352 : 891-899

BARBosA, L.R., O. DÍAZ \& R.G. BARBer (1989): Effects of deep tillage on soil properties, growth and yields of Soya in a compacted Ustochrept in Santa Cruz, Bolivia. - Soil Tillage and Research $15: 51-63$

Barros Ferraz, S.F.D., C.A. Vettorazzi, D.M. Theobald \& M.V. Ramos Ballester (2005): Landscape dynamics of Amazonian deforestation between 1984 and 2002 in central Rondônia, Brazil: assessment and future scenarios. - Forest Ecology and Management $204: 67-83$

BAtJES, N.H. (1998): Mitigation of atmosperic CO2 concentrations by increased carbon sequestration in the soil. - Biology and Fertility of Soils $27: 230-235$

BAtJes, N.H. \& J.A. DiJKshoorn (1999): Carbon and nitrogen stocks in the soils of the Amazon Region. - Geoderma 89 (3) : 273-286

Bayer, C., J. Mielniczuk, T.J.C. Amado, L. Martin-Neto \& S.V. Fernandes (2000a): Organic matter storage in a sandy clay loam Acrisol affected by tillage and cropping systems in southern Brazil. - Soil Tillage and Research 54 : 101-109

Bayer, C., J. Mielniczuk, T.J.C. Amado, L. Martin-Neto \& S.V. Fernandes (2000b): Effect of no-till cropping systems on soil organic matter in a sandy clay loam Acrisol from southern Brazil monitored by spin resonance and nuclear magnetic resonance. - Soil Tillage and Research $53: 95-104$

Bayer, C., L. Martin-Neto, J. Mielniczuk, C.N. Pillon, \& L. SANGOI. (2001): Changes in Soil Organic Matter Fractions under Subtropical No-Till Cropping Systems. - Soil Science Society of America Journal 65 : 1473-1478

BAZZAZ, F.A. (1998): Tropical forests in a future climate: Changes in biological diversity and impact on the global carbon cycle. - Climatic Change 39 : 317-336 
Bell, M.J., G.R. HARCH \& B.J. BRIDGE (1995): Effects of continuous cultivation on Ferrosols in subtropical Southeast Queensland. I. Site characterization, crop yields and soil chemical status. - Australian Journal of Agricultural Research 46 : 237-253

BERLI, M. (2001): Compaction of agricultural subsoils by tracked heavy construction machinery. - Dissertation Swiss Federal Institute of Technology Zürich, ETH Zürich, Diss. No. 14132,108 pp.

Bernoux, M., M. DA ConceiçÃo Santana CARvalho, B. Volkoff \& C.C. Cerri (2002): Brazil's soil carbon stocks. - Soil Science Society of America Journal 66 : 888-896

Beutler, A.N. \& J.F. CENTURION (2004): Soil compaction and fertilization in soybean productivity. - Sciencia Agric. (Piracicaba, Braz.) 61 (6) : 626-631

Bielders, C.L., K. Michels \& J.-L. Rajot (2000): On-Farm Evaluation of Ridging and Residue Management Practices to Reduce Wind Erosion in Niger. - Soil Science Society of America Journal 64 : 1776-1785

Bishop, T. \& A.B. MCBRATNEY (2001): A comparison of prediction methods for the creation of field-extent soil property maps. - Geoderma 103 (1-2) : 149-160

BitTelli, M., G.S. CAMPBell \& M. FluRY (1999): Characterization of particle-size distribution in soils with a fragmentation model. - Soil Science Society of America Journal $63: 782-788$

BlaschKe, C. (1998): Aktuelle Landnutzung und Auswirkungen des traditionellen Brandrodungsfeldbaus auf die Bodenfruchtbarkeit in Lomerío (Chiquitania), Bolivien. unveröffentlichte Diplomarbeit am Geographischen Institut der Universität Göttingen

BlaschKe, P.M., N.A. TRUSTRUM \& D.L. Hicks (2000): Impacts of mass movement erosion on land productivity: a review. - Progress in Physical Geography 24 (1) : 21-52

Blumfield, T.J., Z.H. XU \& C. CHEN (2005): Mineral nitrogen dynamics following soil compaction and cultivation during hoop pine plantation establishment. - Forest Ecology and Management $204: 129-135$

BÖHNER, J. \& T. SELIGE (2004): Spatial prediction of soil parameter using terrain analyses, climate regionalisation and remote sensing. - Geoderma (submitted)

BÖHNER, J. (2004): Regionalisierung bodenrelevanter Klimaparameter für das Niedersächsische Landesamt für Bodenforschung (NLfB) und die Bundesanstalt für Geowissenschaften und Rohstoffe (BGR). - Arbeitshefte Boden 4 : 17-66

BÖHNER, J. \& R. KÖTHE (2003): Bodenregionalisierung und Prozessmodellierung: Instrumente für den Bodenschutz. - Petermanns Geographische Mitteilungen 147 : 68-78

BÖHNER, J., R. KÖTHE \& T. SELIGE (2003a): Geographical Information Systems: Applications to Soils. - in: Hillel, D., C. Rosenzweig, D. Powlson, K. Scow, M. Singer \& Sparks, D. [Eds.]: Encyclopedia of Soils in the Environment - Elsevier, Oxford, pp. 121-129

BöHner, J., SchäFer, W., CONRAD, O., Gross, J. \& Ringeler, A. (2003b): The WEELS Model: Methods, Results and Limitations. - Catena 52 : 289-308.

Böhner, J., R. Köthe, O. Conrad, J. Gross, A. Ringeler \& T. Selige (2002): Soil Regionalisation by Means of Terrain Analysis and Process Parameterisation. - in: MichELI, E., F. Nachtergaele \& L. Montanarella [Eds.]: Soil Classification 2001. European Soil Bureau, Research Report 7 : 213-222 
Bonde, T.A., B.T. Christensen \& C.C. CERri (1992): Dynamics od soil organic matter as reflected by natural $13 \mathrm{C}$ abundance in particle size fractions of forested and cultivated Oxisols. - Soil biology \& biochemistry 24 (3) : 275-277

Botta, G.F., D. JoraJura, R. BALbuena \& H. Rosatto (2004): Mechanical and cropping behavior of direct drilled soil under different traffic intensities: effect on soybean (Glycine $\max$ L.) yields. - Soil \& Tillage Research 78 : 53-58

Bounoua, L., R. S. DeFries, M. L. ImHoff, \& M. K. Steininger (2003): Land use and local climate: A case study near Santa Cruz, Bolivia. - Meteorology and Atmospheric Physics, Vol.86, No. 1-2, S.73-85.

Bourennane, H., D. King \& A. Couturier (2000): Comparison of kriging with external drift and simple linear regression for predicting soil horizon thickness with different sample densities. - Geoderma 97 (3-4) : 255-271

BRAimoH, A.K. \& P.L.G. VLEK (2004): The impact of land-cover change on soil properties in northern Ghana. - Land Degradation and Development 15 : 65-74

BRIDGES, E.M. \& J.H.V. VAN BAREN (1997): Soil: an overlooked, undervalued and vital part of the human environment. - The Environmentalist $17: 15-20$

BRONICK, C.J. \& R. LAL (2005): Soil structure and management: a review. - Geoderma $124: 3-22$

BRown, S. \& A.E. Lugo (1990): Effects of forest clearing and succession on the carbon and nitrogen content of soils in Puerto Rico and US Virgin Islands. - Plant and Soil 124 : 401414

BUCHAN, G.D. (1989): Applicability of the simple lognormal model to particle-size distribution in soils. - Soil Science $147: 155-161$

Burke, I.C., W.K. LAUERnRoth \& D.P. Coffin (1995): Soil organic matter recovery in semiarid grasslands: implications for the conservation reserve program. - Ecological Monographs 5 : 793-801

Burle, M.L, J. MielniCZUK \& S. FOCCHI (1997): Effect of cropping systems on soil chemical characteristics, with emphasis on soil acidification. - Plant and Soil 190 : 309-316

Burrough, P.A. \& S.R. YAtes (1994): The state of the art in pedometrics. - Geoderma 62 : 311-326

Buschiazzo, D.E., T.M. Zobeck \& S.B. Aimar (1999): Wind erosion in loess soils of the semiarid argentinian pampas. - Soil Science 164(2) : 133-138

Camacho, M.O., W.Q. Corsero, T.I. Martínez \& D.M. Rojas (2001): Tasa de Deforestación del Departamento de Santa Cruz, Bolivia 1993-2000. - Proyecto de Manejo Forestal Sostenible (BOLFOR), Superintendencia Forestal, Santa Cruz, Bolivia

CANillas, C.C. \& V.M. SAlokHE (2001): Regression analysis of some factors influencing soil compaction. - Soil Tillage and Research $61: 167-178$

CAO (2002): Numeros de nuestra tierra. Evaluación de desempeño del sector agropecuario. Cámara Agropecuaria del Oriente, Santa Cruz

CARDille, J.A. \& J.A. FOLEY (2002): Characterizing patterns of agricultural land use in Amazonia by merging satellite classifications and census data. - Global Biogeochemical Cycles 16 (3) : (10.1029/2000GB001386) 
CARDille, J.A. \& J.A. Foley (2003): Agricultural land-use change in Brazilian Amazônia between 1980 and 1995: Evidence from integrated satellite and census data. - Remote Sensing of Environment $87: 551-562$

Castro, N.M.D.R., A.-V. Auzet, P. Chevallier \& J.-C. LePrun (1999): Land use change effects and erosion from plot to catchment scale on the basaltic plateau of Southern Brazil. - Hydrological processes $13: 1621-1628$

Centurion, J.F., A.N. Beutler \& Z. Menezes de Souza (2004): Physical Attributes of kaolinitic and oxidic Oxisols resulting from different Usage Systems. - Brazilian Archives of Biology and Technology 47 (5) : 725-732

CERDÀ, A. (2000): Aggregate stability against water forces under different climates on agriculture land and scrubland in southern Bolivia. - Soil Tillage and Research 57 : 159166

Cerri, C.E.P., M. Bernoux, V. Chaplot, B. Volkoff, R.L. Victoria, J.M. Melillo, K. PAUSTIAN \& C.C. CERRI (2004a): Assessment of soil property spatial variation in an Amazon pasture: basis for selecting an agronomic experimental area. - Geoderma 123 : 5168

Cerri, C.E.P., C.C. Cerri, K. Paustian, M. Bernoux \& J.M. Mellilo (2004b): Combining soil $\mathrm{C}$ and $\mathrm{N}$ variability and modelling approaches for measuring and monitoring soil carbon sequestration. - Environmental Management 33 (1) : 274-288

CHEPIL, W.S. (1941): Relation of wind erosion to the dry aggregate structure of a soil. Scientific Agriculture $21: 488-507$

CHEPIL, W.S. (1952): Improved rotary sieve for measuring state and stability of dry soil structure. - Soil Science Society of America Proceedings 16 : 113-117

ChepIL, W.S. \& N.P. WoOdRUfF (1963): The Physics of wind erosion and its control. Advances in Agronomy $15: 211-302$

Chomitz, K.M. \& D.A. Gray (1996) Roads, land use, and deforestation: a spatial model applied to Belize. - World Bank Economic Review 10 : 487-512

CIAT (1980): Efecto de los cultivos contínuos sobre la fertilidad del suelo en las áreas de colonización de Santa Cruz. - Centro de Investigación Agricola Tropical (CIAT), Boletín Especial 21, Santa Cruz, Bolivia

Cochrane, T.T. (1979): Manganese and zinc deficiencies in sugar-cane grown in Entisols near Santa Cruz. - Tropical Agriculture 56 (3) : 219-224

Coelho, C.A.S., C.B. Uvo \& T. Ambrizzi (2002): Exploring the impacts of tropical Pacific SST on the precipitation patterns over South America during ENSO periods. - Theoretical and Applied Climatology $71: 185-197$

Cogle AL, J. Littlemore \& D.H. Heiner (1995) Soil organic matter changes and crop responses to fertilizer under conservation cropping systems in the semi-arid tropics of North Queensland, Australia. - Australian Journal of Experimental Agriculture 35 : 233237.

CordecruZ, KFW \& CONSORCIO IP/CES/KWC (1994): Monitoreo de Bosques con ayuda de la evaluación de datos de satélite, periodo 1988/89-1991. - Proyecto de Proteccion de los Recursos Naturales en el Departamento de Santa Cruz (Componente Proyecto Tierras Bajas)

Cordecruz, KFW \& Consorcio IP/CES/KWC (1996): Memoria del PLUS. Plan de Uso del Suelo del Departamento de Santa Cruz, Bolivia. Memoria Esplicativa de la Propuesta 
Técnica a la Subcomisión de Uso del Suelo. - Cooperación Finanziera del Gobierno Alemán, Prefectura del Departamento, Consorcio IP/CES/KWC; Santa Cruz, Bolivia

Cramer, W., A. Bondeau, S. Schaphoff, W. Lucht, B. Smith \& S. Sitch (2004): Tropical forests and the global carbon cycle: Impacts of atmospheric carbon dioxide, climate change and rate of deforestation. - Philosophical Transactions of The Royal Society B 359 : 331343

CRASWELL, E.T. \& R.D.B. LEFroy (2001): The role and function of organic matter in tropical soils. - Nutrient Cycling in Agroecosystems 61 : 7-18

CUMAT (1992): Desbosque de la Amazonia Boliviana. - Capacidad de Uso Mayor de la Tierra, La Paz, Bolivia

Da Silva, E.M., J.E.F.W. Lima, L.N. Rodrigues \& J.A. DE Azevedo (2004): Comparação de modelos matemáticos para o traçado de curves granulométricas. - Pesquisa Agropecuária Brasileira 39 (4) : 363-370

DADDOW, R.L. \& G.E. WARRINGTON (1983): Growth-limiting soil bulk densities as influenced by soil texture. - USDA Forest Service Watershed Systems Development Group Report WSDG-TN-00005

DALAL, R.C. \& R.J. MAYER (1986a): Long-term trends in fertility of soils under continuous cultivation and cereal cropping in Southern Queensland. I. Overall changes in soil properties and trends in winter cereal yields. - Australian Journal of Soil Research 24 : 265-279

DALAL, R.C. \& R.J. MAYER (1986b): Long-term trends in fertility of soils under continuous cultivation and cereal cropping in Southern Queensland. V. Rate of loss of total nitrogen from the soil profile and changes in carbon : nitrogen ratios. - Australian Journal of Soil Research 37 : 493-504

DALAL, R.C. \& K.Y. CHAN (2001): Soil organic matter in rainfed cropping systems of the Australian cereal belt. - Australian Journal of Soil Research 39 : 435-464

DAlal, R.C., B.P. HARMS, E. KRULl \& W.J. WANG (2005): Total soil organic matter and its labile pools following mulga (Acacia aneura) clearing for pasture development and cropping 1. Total and labile carbon. - Australian Journal of Soil Research 43 : 13-20

D’Andrea, A.F., M.L. Naves Silva, N. Curi \& L.R. Guimarães Guilherme (2004): Estoque de carbono e nitrogênio e formas de nitrogênio mineral em um solo submetido a diferentes sistemas de manejo. - Pesquisa Agropecuária Brasileira 39 (2) : 179-186

DAVIDSON, E.A. \& I.L. ACKERMAN (1993): Changes in soil carbon inventories following cultivation of previously untilled soils. - Biogeochemistry $20: 161-193$

DAVIES, P. (1996): Valuing soils in the tropical lowland of Eastern Bolivia. - in: ABELSON, P. [Ed.]: Project Appraisal and Valuation of the Environment, General Principles and Six Case Studies in Developing Countries : 240-267, Macmillan Press, London

DERPSCH, R. (1974): Estudio sobre la erosión eólica en la región integrada del Departamento de Santa Cruz y medidas prácticas para su control. - Cooperación Boliviano-Alemana, La Paz, Bolivia

DERPSCH, R. (2000): Direktsaat: Nachhaltige Landwirtschaft durch Verzicht auf Bodenbearbeitung. - Entwicklung und Ländlicher Raum 4

DERPSCH, R. (2001): Keynote: Frontiers on conservation tillage and advances in conservation practice - in: StotT, D.E., R.H. MohtAR \& G.C. SteInHARDT [Eds.]: Sustaining the global 
farm : 248-254, $10^{\text {th }}$ International Soil Conservation Organization Meeting, Purdue University

DERPSCH, R. (2002): Making conservation tillage conventional, building a future on 25 years of research: Research and extension perspective. - in: SANTEN VAN, E. [Ed.]: conservation tillage conventional, building a future on 25 years of research. - Proc. Of $25^{\text {th }}$ Annual Southern Conservation Tillage Conference for Sustainable Agriculture. Special Report No. 1, Alabama Agric. Expt. Stn. And Auburn University

Desjardins, T., E. Barros, M. Sarrazin, C. Girardin \& A. Mariotti (2004): Effects of forest conversion to pasture on soil carbon content and dynamics in Brazilian Amazonia. Agriculture, Ecosystems and Environment 103 : 365-373

DíAZ, O. \& R.G. BARBER (1988): Effects of a grazed legume fallow and a forest fallow on the physical and chemical properties of an alluvial soil at Chané. - Centro de Investigación Agricola Tropical (CIAT) Report 49, Santa Cruz, Bolivia.

Dinerstein, E.D., M. Olson, D.J. Graham, A.L. Webster, A.A. Rim, M.P. Bookbinder \& G. LEDEC, (1995): A conservation assessment of the terrestrial ecoregions of Latin America and the Caribbean. World Bank, Washington DC, 129 pp.

Dominy C. S., R. J. HAYNeS \& R. V. ANTWERPEN (2002): Loss of soil organic matter and related soil properties under long-term sugarcane production on two contrasting soils. Biology and Fertility of Soils $36: 350-356$

DONG, Z.B., X.M. WANG \& L.Y. LIU (2000): Wind erosion in arid and semiarid China: an overview. - Journal of Soil and Water Conservation $55: 439-444$

Enej, A.E., A. Agboola, E.A. Aiyelari, T. Honna, S. Yamamoto, M. Irshad \& T. Endo (2003): Soil physical and micronutrient changes following clearing of a tropical rainforest. - Journal of Forest Research $8: 215-219$

Fabiola, N., B. Giarola, A.P. DA Silva, S. Imhoff \& A.R. DeXTer (2003): Contribution of natural soil compaction on hardsetting behavior. - Geoderma 113 (1) : 95-108

FAO (1998): Soil and Terrain Database for Latin America and the Caribbean. Edition 1.0 Food and Agriculture Organization of the United Nations (FAO), Rom

FAO (2003): State of the World's forest 2003. - Food and Agriculture Organization of the United Nations, Rome $151 \mathrm{pp}$.

FEARNSIDE, P.M. (2001): Soybean cultivation as a threat to the environment in Brazil. Environmental Conservation 28 (1) : 23-38

FEARNSIDE, P.M. \& R.L. BARBosa (1998): Soil carbon changes from conversion of forest to pasture in Brazilian Amazon. - Forest Ecology and Management 108 : 147-166

FEGASACRUZ (1999): Memoria 1996-1998. - Federación de Ganaderos de Santa Cruz

FIFER, J. V. (1982): The search for a series of small successes: frontiers of settlement in eastern Bolivia. - Journal of Latin American Studies 14 : 407-432

FRANZLUEBBERS, A.J. (2002): Soil organic matter stratification ration as an indicator of soil quality. - Soil \& Tillage Research 66 : 95-106

Fredlund, M.D., D.G. Fredlund \& G.W. Wilson (2000): An equation to represent grainsize distribution - Canadian Geotechnical Journal 37 (4) : 817-827

Fryrear, D.W., P.L. Sutherland, G. Davies, G. Hardee \& M. Dollar (2001): Wind erosion estimates with RWEQ and WEQ. - in: STOTT, D.E., R.H. MohtAR \& G.C. 
SteINHARDT [Eds.]: Sustaining the global farm : $760-765,10^{\text {th }}$ International Soil Conservation Organization Meeting, Purdue University

Gaugin, M., T. Marienhof, S. BJoholm \& C.J. Austin (2002) : Nutrient dynamics in shifting cultivation systems of the humid tropics. - Course report in IPNS (Integrated Plant Nutrients \& Soil Fertility in the Tropics), KVL, The Royal Veterinary and Agricultural University Copenhagen, Denmark

GeHrt, E. \& J. BöHner (2002): Vom Punkt zur Fläche. Probleme des upscaling in der Bodenkartierung. - Diskussionsforum Bodenwissenschaften: Vom Bohrstock zum Bildschirm : 17-34

GEIST, H.J. \& E. LAMBIN (2001): What drives tropical deforestation? A meta-analysis of proximate and underlying causes of deforestation based on subnational case study evidence. - LUCC Report Series 4

GEROLD, G. (1986): Klimatische und pedologische Bodennutzungsprobleme im ostbolivianischen Tiefland von Santa Cruz. - in: BuchHOLz, H.J. [Ed.]: Jahrbuch der Geographischen Gesellschaft Hannover : 69-162

GEROLD, G. (1988): Zur Anwendung von Schätzmodellen der Abspülresistenz tropischer Böden bei Neulanderschließungen am Beispiel der Äußeren Tropen Boliviens. - Jahrbuch der Geographischen Gesellschaft Hannover : 161-188

GEROLD, G. (1991a): Klimatische und pedologische Bodennutzungsprobleme in tropischen Waldgesellschaften (Bolivien). - Gießener Beiträge zur Land- und Forstwirtschaft in den Tropen und Subtropen $60: 91-110$

GEROLD, G. (1991b): Nutzungseingriffe in tropische Waldgesellschaften und deren pedoökologische Folgen (Bolivien). - Gießener Beiträge zur Entwicklungsforschung I (19) : 25-40

Gerold, G. (1991c): Human impact on Forest Ecosystems and Soil Deterioration in Tropical Bolivia. - in: ERdelen, W., N. IshWARAN \& P. MÜller [Eds.]: Tropical Ecosystems : 107-120, Markgraf Scientific Books, Weikersheim

Gerold, G. (2001a): The pedo-ecological consequences of traditional and mechanised landuse-systems in the lowlands of Bolivia (Dep. of Santa Cruz). - Proceedings of ICLD3, Session II, Extended Abstract, 17

GEROLD, G. (2001b): The pedo-ecological consequences of different landuse-systems in the lowlands of Bolivia (Dep. of Santa Cruz). - in: GARCIA-Torres, L., J. BENITES \& A. MARTINEZ-VILEDA [Eds.]: Conservation Agriculture, A Worldwide Challenge $2: 275-279$, ECAF, Madrid

GEROLD, G. (2002a): Traditional and modern soil management techniques for sustainable landuse in the lowlands of Bolivia (Santa Cruz) - in: FAZ, A., R. ORTIZ \& A.R. MERMUT [Eds.]: Sustainable use and management of soils in arid and semiarid regions. SUMASS $2002: 130-147$

GEROLD, G. (2002b): Geoökologische Grundlagen nachhaltiger Landnutzungssysteme in den Tropen. - Geographische Rundschau 54 (5)

Gessler, P.E., O.A. Chadwick, F. Chamran, L. Althouse, \& K. Holmes (2000): Modeling Soil -Landscape and Ecosystem Properties Using Terrain Attributes. - Soil Science Society of America Journal 64 : 2046-2056 
Gomes, L., J.L. Arrúe, M.V. López, G. Sterk, D. Richard, R. Gracia, M. Sabre, A. GAUDICHET \& J.P. FRANGI (2003): Wind erosion in semiarid agricultural area of Spain: the WELSON project. - Catena $52: 235-256$

Goovaerts, P. (1999): Geostatistics in soil science: state-of-the-art and perspectives. Geoderma 89 (1- 2) : 1-45

GORRIN, A. (2005): Knowledge-based monitoring and evaluation system of land use: assessing the ecosystem conservation status in the influence area of a gas pipeline in Bolivia. - PhD-thesis, University of Goettingen 169 pp.

GROß, J. (2002): Quantifizierung winderosionsbedingter Staubausträge in Agrarlandschaften Nieder-sachsens. - Geosynthesis 12, Hannover, 142 pp.

GuAman, A. (1988a): Estudio de suelos - Levantamiento integrado de recursos naturales sector San Julian. - Cooperación regional de desarrollo de Santa Cruz. Santa Cruz de la Sierra

Guaman, A. (1988b): Estudio de suelos - Levantamiento integrado de recursos naturales sector Pailon, Pozo del Tigre, Los Troncos. - Cooperación regional de desarrollo de Santa Cruz. Santa Cruz de la Sierra

Guaman, A. (1990): Problemas de fertilidad y fertilizantes en los suelos de Santa Cruz. - in : FAO - Proyecto fertilizantes GCPF/Bol/018/NET: Seminario nacional sobre fertilidad de suelos y uso de fertilizantes en Bolivia. - Centro de Investigación Agricola Tropical (CIAT), Instituto Boliviano de Tecnología Agropecuaria (IBTA), Santa Cruz

Guaman, A. (1999): Estudio de suelos del municipio Cabezas. - Cooperación tecnica boliviana alemana. Santa Cruz de la Sierra

GUO, L.B. \& R.M. GIFFORD (2002): Soil carbon stocks and land use change: a meta analysis. Global Change Biology $8: 345-360$

HajabBasi, M.A., A. Jalalian \& H.R. KarimzadeH (1997): Deforestation effects on soil physical and chemical properties, Lordegan, Iran. - Plant and Soil 190 : 301-308

HAMBURG, S.P. (2000): Simple rules for measuring changes in ecosystem carbon in forestryoffset projects. - Mitigation and Adaption Strategies for Global Change 5 : 25-37

Hansen, M.C., R.S. Defries, J.R.G. Townshend \& R. SOHLberg (2000): Global land cover classification at $1 \mathrm{~km}$ spatial resolution using a classification tree approach. - International Journal of Remote Sensing 21 (6,7) : 1331-1364

HARTEMINK, A.E. (1998): Soil chemical and physical properties as indicators of sustainable land management under sugar cane in Papua New Guinea. - Geoderma 85 : 283-306

HASSINK, J. (1997): The capacity of soils to preserve organic $\mathrm{C}$ and $\mathrm{N}$ by their association with clay and silt particles. - Plant and Soil 191 : 77-87

Henderson B.L., E.N. BUi, C.J. Moran \& D.A.P. SimON (2004): Australia-wide predictions of soil properties using decision trees. - Geoderma $124: 383-398$

Hengl, T., D.G. Rossiter \& A. SteIn (2003): Soil sampling strategies for spatial prediction by correlation with auxiliary maps. - Australian Journal of Soil Research 41 : 1403-1422

Hengl, T., G.B.M. Heuvelink, A. Stein (2004): A generic framework for spatial prediction of soil variables based on regression-kriging. - Geoderma $120: 75-93$

HeuvelinK, G.B.M., R. WebSTER (2001): Modelling soil variation: past, present, and future. - Geoderma $100: 269-301$ 
Hwang, S.I., K.P. LeE, D.S. LeE \& S.E. Powers (2002): Models for estimating soil particlesize distributions. - Soil Science Society of America Journal 66 : 1143-1150

IADB (2000): Strategic Environmental Assessment of the Santa Cruz-Puerto Suarez Corridor, Bolivia. -Final Report, Inter-American Development Bank (IADB). Consortium PRIME Engenharia, Museo Noel Kempff Mercado, Ascociación POTLACH

IBISCH, P.L., K. Columba \& S. Reichle [eds.] (2002): Plan de Conservación y Desarrollo Sostenible para el Bosque Seco Chiquitano, Cerrado y Pantanal Boliviano. - Editorial FAN, Santa Cruz, Bolivia

IBISCH, P.L., S. G. BECK, B. GERKMAN \& A. CARRETERO (2004): Ecoregions and ecosystems. - in: IBISCH, P.L. \& G. MÉRIDA [eds.]: Biodiversity: The richness of Bolivia. State of knowledge and conservation : 47-86. Editorial FAN, Santa Cruz de la Sierra, Bolivia

ImHOFF, S., A.P. DA Silva \& D. FALlow (2004): Susceptibility to compaction, load support capacity and soil compressibility of Hapludox. - Soil Science Society of America Journal $68: 17-24$

INE (2002): Estadísticas del Departamento de Santa Cruz 2002. - Instituto Nacional de Estatistica, Republica de Bolivia

INE (2004): Anuario Estadístico 2003. - Instituto Nacional de Estatistica, Republica de Bolivia

IPORRE, J.B. (1996): Estudio de Suelos en dos Areas de Accion Forestal Zona de Lomerio. Documento Técnico 28, Proyecto BOLFOR, Santa Cruz

IRWIN, E.G. \& J. GEOGHEGAN (2001): Theory, data, methods: developing spatially explicit economic models of land use change. - Agriculture, Ecosystems and Environment 85 : 7 23

Ishida, T., S. ITAGaKi, Y. SASAKI \& H. ANDO (2003): Drainage Network Analysis for Regional Partitions of Alluvial Paddy-Field Soils. - Soil Science Society of America Journal 67 : 190-197

JAIYEOBA, I.A. (2003): Changes in soil properties due to continuous cultivation in Nigerian semiarid Savannah. - Soil Tillage and Research 70 : 91-98

Jantalia, C.P., H. Pereira dos Santos, J.E. Denardin, R. Kochhann, B.J. Rodrigues Alves, S. URquiaga \& R.M. Boddey (2003): Influência de rotações de culturas no estoque de carbono e nitrogênio do solo sob plantio direto e preparo convencional. Agronomia 37 (2) : 91-97

JENNY, H. (1941): Factors of soil formation. A system of quantitative pedology. - McGrawHill, New York

Jensen, J. R. (1996): Introductory Digital Image Processing: A Remote Sensing Perspective. Prentice Hall, $2^{\text {nd }}$ edition, $316 \mathrm{pp}$.

Kaimowitz, D. \& A. Angelsen (1998): Economic Models of Tropical Deforestation: A Review. Center for International Forestry Research (CIFOR), Bogor

Kaimowitz, D., C. Vallejos, P. Pacheco \& R. Lopez (1998). Municipal governments and forest management in lowland Bolivia. - Journal of Environment and Development 7 (1) : $45-60$

Kaimowitz, D., Thiele \& P. PACheco (1999): The effects of structural adjustment on deforestation and forest degradation in lowland Bolivia. - World Development 27 : 505520 
KAImowitz, D \& J. SMith (2001): Soybean technology and the loss of natural vegetation in Brazil and Bolivia. - in: KAIMOWITZ \& ANGELESEN [Eds.]: Agricultural technologies and tropical deforestation : 195-211, CAB International

Kaimowitz, D., P. Mendez, A. Puntodewo \& J. Vanclay (2002): Spatial regression analysis of deforestation in Santa Cruz, Bolivia. - in: Wood, C.H. \& R. PorRo [Eds.]: Deforestation and land use in the Amazon : 41-65, University Presses of Florida, Gainesville

Kalivas, D.P., D.P. Triantakonstantis, V.J. Kollias (2002): Spatial prediction of two soil properties using topographic information. - Global Nest: The International Journal 4 : 41-49

Kalnay, E., Kanamitsu, M., Kistler, R., Collins, W., Deaven, D., Gandin, L., Iredell, M., Saha, S., White, G., Woollen, J., Zhu, Y., LeetmaA, A., Reynolds, R., Chelliah, M., Ebisuzaki, W., Higgins, W., Janowiak, J., Mo, K., Ropelewski, C., Wang, J., JENne, R. \& JosePH, D. (1996): The NCEP/NCAR 40-Year Reanalysis Project. - Bulletin of the American Meteorological Society 03

KANE, R.P. (1999): Rainfall extremes in some selected parts of Central and South America: ENSO and other relationships reexamined. - International Journal of Climatology 19 : 423455

KARsten, R. (2000): Pedoökologische Auswirkungen des Brandrodungsfeldbaus in den wechselfeuchten Tropen (Santa Cruz - Bolivien). - Diplomarbeit Geographisches Institut Universität Göttingen (unveröffentlicht)

KATYAL, J.C., N.H. RAO \& M.N. REDDY (2001): Critical aspects of organic matter management in the Tropics: the example of India. - Nutrient Cycling in Agroecosystems 61 : 77-88

KAYOMBO, B. \& R. LAL (1994): Responses of Tropical Crops to Soil Compaction. - in: SoAne, B.D. \& C. VAN Ouwerkerk [Eds.]: Soil Compaction in Crop Production : 287315, Amsterdam

KELLER, T. (2004): Soil compaction and soil tillage - studies in agricultural soil mechanics. PhD-thesis, Swedish University of Agricultural Sciences, Uppsala, Agraria 489, 75 pp.

Kemp, A. (2005): Niederschlagsvariabilität und Ertragsrisiko im Tiefland von Santa Cruz (Bolivien). - Diplomarbeit Geographisches Institut Universität Göttingen (unveröffentlicht)

KenNARD, D.K. \& H.L. GHOLZ (2001): Effects of high- and low-intensity fires on soil properties and plant growth in a Bolivian dry forest. - Plant and Soil 234 : 119-129

KENNARD, D.K. (2002): Secondary forest succession in a tropical dry forest: patterns of development across a 50-year chronosequence in lowland Bolivia. - Journal of Tropical Ecology $18: 53-66$

Kidman, D.C., K.A AdAms, E. OrTíZ, \& J. NAVARro (1980): The effect of continuous cropping on soil fertility in the colonization areas of Santa Cruz. - in: Centro de Investigación Agricola Tropical and Consortium for International Developement, Boletín Especial 21, Santa Cruz, Bolivia

Kidman, D.C., K.A. Adams, E. Ortiz \& J. Navarro (1980): Efecto de los cultivos contínuos sobre la fertilidad del suelo en las áreas de colonización de Santa Cruz. - Centro de Investigación Agricola Tropical, Boletín Especial 21, Santa Cruz, Bolivia

KilleEn, T.J. (1990): The grasses of Chiquitanía, Santa Cruz, Bolivia. - Annals Missouri Botanical Garden 77 : 125-201 
KilleEn, T.J., A. JARDIM, F. MAMANI \& N. RoJAS (1998): Diversity, composition, structure, and biomass estimates of a tropical semideciduous forest in the Chiquitanía region of Santa Cruz, Bolivia. - Journal of Tropical Ecology 14: 803-827

KILleEN, T.J., B.T. LOUMAN \& T. GRIMWOOD (1990): La ecologia paisajistica de la region de Concepción y Lomerio en la provincia de Ñuflo de Chavez, Santa Cruz, Bolivia. Ecología en Bolivia $16: 1-45$

KnOtTers, M., D. Brus, J. VoshaAR (1995): A comparison of kriging, co-kriging and kriging combined with regression for spatial interpolation of horizon depth with censored observations. - Geoderma $67(3,4): 227-246$

KoK, K. \& A. VELDKAMP (2001): Evaluating impact of spatial scales on land use pattern analysis in Central America. - Agriculture, Ecosystems and Environment 85 : 205-221

KozlowsKi, T.T. (1999): Soil Compaction and Growth of Woody Plants. - Scandinavian Journal of Forest Research 14 : 596-619

Kuntze, H., G. Roeschmann \& G. Schwerdtfeger (1988): Bodenkunde. - UniTaschenbücher 1106, 4. Aufl., Ulmer : Stuttgart

KunBach, T.M. (1997): Estudio de Suelos: Zona Centro Izozog (Cmd. Ivasiriri), Provincia Cordillera - Departamento Santa Cruz - Bolivia. - unveröffentlichte Studie APCOB Santa Cruz, Universität Göttingen Geographisches Institut

LAAKE, VAN P.E. \& G.A. SÁNCHEZ-AzOFEIFA (2004): Focus on deforestation: zooming in on hot spots in highly fragmented ecosystems in Costa Rica. - Agriculture, Ecosystems and Environment $102: 3-15$

LAL, R. \& T. J. LOGAN (1995): Agricultural activities and greenhouse gas emissions from soils of the tropics. - in: LAL, R., J.M KIMBLE, E. LeVINE \& B.A. STEWART [Eds.]: Soil Management and Greenhouse Effect : 293-307, CRC/Lewis Publishers, Boca Raton

LAL, R. (1990): Soil Erosion in the Tropics, Principles \& Management. - McGraw-Hill, Inc., New York

LAL, R. (1995): Tillage systems in the tropics: Management options and sustainability implications. - FAO Soils Bulletin 71

LAL, R. (1996a): Deforestation and land-use effects on soil degradation and rehabilitation in western Nigeria. I. Soil physical and hydrological properties. - Land degradation and development $7: 19-45$

LAL, R. (1996b): Deforestation and land-use effects on soil degradation and rehabilitation in western Nigeria. II. Soil chemical properties. - Land degradation and development 7 : 8798

LAL, R. (1996c): Deforestation and land-use effects on soil degradation and rehabilitation in western Nigeria. III. Runoff, soil erosion and nutrient loss. - Land degradation and development $7: 99-119$

LAL, R. (1997): Degradation and resilience of soils. - Philosophical Transactions of The Royal Society B 352 : 997-1010

LAL, R. (1998): Soil quality changes under continuous cropping for seventeen seasons of an Alfisol in Western Nigeria. - Land degradation and development 9 : 259-274

LAL, R. (2000): Physical management of soils of the tropics: priorities for the $21^{\text {st }}$ century. Soil Science 165 : 191-207 
LAL, R. (2001): Soil degradation by erosion. - Land degradation and development 12 : 519539

LAL, R. (2002): Soil carbon dynamics in cropland and rangeland. - Environmental Pollution $116(3): 353-362$

LAL, R. (2003): Offsetting global CO2 emissions by restoration of degraded soils and intensification of world agriculture and forestry. - Land degradation and Development 14 : 309-322

Lambin, E.F., B.L. Turner, H.J. Geist, S.B. Agbola, A. Angelsen, J.W. Bruce, O.T. Coomes, R. Dirzo, G. Fischer, C. Folke, P.S. George, K. Homewood, J. Imbernon, R. LeEmans, X. Li, E.F. Moran, M. Mortimore, P.S. Ramakrishnan, J.F. Richards, H. SkÅnes, W. Steffen, G.D. Stone, U. Svedin, T. Veldkamp, C. Vogel \& J. Xu (2001): The causes of land-use and land-cover change: moving beyond the myths. - Global Environmental Change $11: 261-269$

Lampurlanés, J. \& C. CAntero-Martínez (2003): Soil Bulk Density and Penetration Resistance under Different Tillage and Crop Management Systems and Their Relationship with Barley Root Growth. - Agronomy Journal 95 : 526-536

LANDERS, J.N. (2001): How and why the Brazilian zero tillage explosion occurred. - in: Stott, D.E., R.H. Mohtar \& G.C. Steinhardt [Eds.]: Sustaining the global farm : 29$39,10^{\text {th }}$ International Soil Conservation Organization Meeting, Purdue University

LANDON, J.R [Ed.] (1984): Booker Tropical Soil Manual. A handbook for survey and agricultural land evaluation in the tropics and subtropics. - Booker Agriculture International Limited

LAurance, W. F., \& R. O. BierregaARD (1997): Tropical forest remnants: ecology, management and conservation of fragmented communities. - University of Chicago Press, Chicago

LAWTON, R.O., U.S. NAIR, R.A. PIELKE \& R.M. WelCh (2001): Climatic impact of tropical lowland deforestation on nearby montane cloud forests. - Science 294 : 584-587

LEAN, J. \& P.R ROWNTREE (1997): Understanding the Sensitivity of a GCM Simulation of Amazonian Deforestation to the Specification of Vegetation and Soil Characteristics. - Journal of Climate 10 (6) : 1216-1235

Leite, L.F.C., E.S. MendonçA, P.L.O.A. Machado \& E.S. Matos (2003): Total C and N storage and organic $\mathrm{C}$ pools of a Red-Yellow Podzolic under conventional and no tillage at the Atlantic forest zone, south-eastern Brazil. - Australian Journal of Soil Research 41 : 717-730

Lemenih, M., E. KARLtun \& M. Olsson (2005): Assessing soil chemical and physical property responses to deforestation and subsequent cultivation in smallholders farming system in Ethiopia. - Agriculture, Ecosystems and Environment 105 : 373-386

LILLESAND, T.M. \& R.W. KIEFER (2000): Remote Sensing and Image Interpretation. - Wiley and Sons, $4^{\text {th }}$ Edition, 724 pp. New York

LLANOS, J.L.R., F.A. LEDEZMA \& P.M. FrANCO (1996): Diagnostico regional sobre siembra directa. - Estudio de campo No. 9, Centro de Investigación Agricola Tropical (CIAT), Santa Cruz, Bolivia

Lobe, I., W. Amelung \& C.C. Du Preez (2001): Losses of carbon and nitrogen with prolonged arable cropping from sandy soils of the South African Highveld. - European Journal of Soil Science 52 : 93-101 
LOVELAND, T.R. \& A.S. BELWARD (1997): The IGBP-DIS global $1 \mathrm{~km}$ land cover data set, DISCover: first results. - International Journal of Remote Sensing 18 : 3289-3295

LOZA, A. (2004): A spatial logistic model for Tropical forest conversion: A Case study of Carrasco province, (1986-2002) Bolivia. - MSc thesis, International Institute for Geoinformation Science and Earth Observation (ITC), Enschede

LUGO, A.E., M.J. SANCHEZ (1986): Land use and organic carbon content of some subtropical soils. - Plant and Soil $96: 185-196$

MAAS, J.M. (1995): Conversion of tropical dry forest to pasture and agriculture. - in: Bullock, S.H., H.A. Mooney \& E. Medina [Eds.]: Seasonally Dry Tropical Forests : 399-422, Cambridge University Press Cambridge

MAChado DE A , P.L.O. \& C. A. Silva (2001): Soil management under no-tillage systems in the tropics with special reference to Brazil. - Nutrient Cycling in Agroecosystems 61 : 119130

MaiA, J.L.T \& M.R. RibeIRo (2004): Propriedades de um Argissolo Amarelo fragipânico de Alagoas sob cultivo contínuo da cana-de-açúcar. - Pesquisa Agropecuária Brasileira 39 (1) : 79-87

MANN, L.K. (1986): Changes in soil carbon storage after cultivation. - Soil Science 142 (5) 279-288

MARKUSSEN, M. (2000): Pedoökologische Folgen des mennonitischen Landnutzungssystems im Oriente Boliviens (Departamento Santa Cruz). - Diplomarbeit Geographisches Institut Universität Göttingen (unveröffentlicht)

MARTínEZ, L. (2004): Potential of agropastoral systems for managing degraded soils in Santa Cruz, Bolivia. - in: CIAT/EMBRAPA [Eds.]: Agropastoral systems for the tropical savannas of Latin America. CIAT Publications 338 : 164-172

Mas, J.F., A. Velázquez, J.R. Díaz-Gallegos, R. Mayorga-Saucedo, C. Alcántara, G. Bocco, R. CAstro, T. Fernández \& A. Pérez-Vega (2004): Assessing land use/cover changes: a nationwide multidate spatial database for Mexico. - International Journal of Applied Earth Observation and Geoinformation 5 : 249-261

MASRENA (2000): Uso y Manejo de Recursos Naturales en Colonia Menonitas. - Manejo Sostenibile de los Recursos Naturales en Santa Cruz. Cooperación Técnica Boliviano Alemania IP/GTZ, Santa Cruz

Matson, P. A., W.J. PARTON, A.G. POWER \& M.J. Swift (1997): Agricultural Intensification and Ecosystem Properties. - Science 277 : 504-509

McAlister, J.J., B.J. SMith \& B. SANChEZ (1998): Forest clearance: impact of landuse change on fertility status of soils from the São Francisco area of Niterói, Brazil. - Land Degradation and Development 9 : 425-440

McBratney, A.B., I. Odeh, T. Bishop, M. Dunbar, T. Shatar (2000): An overview of pedometric techniques of use in soil survey. - Geoderma 97 (3-4) : 293-327

McBratney, A.B., M.L. Mendonça Santos \& B. Minasny (2003): On digital soil mapping. - Geoderma $117: 3-52$

McGrath, D.A., K. Smith, H.L. Gholz \& F. DE Assis Oliveira (2001): Effects of LandUse Change on Soil Nutrient Dynamics in Amazônia. - Ecosystems 4 : 625-645 
MCKenZie, N.J. \& M.P. Austin (1993): A quantitative Australian approach to medium and small scale surveys based on soil stratigraphy and environmental correlation. - Geoderma $57: 329-355$

McKenZIE, N.J. \& P.J. RYAN (1999): Spatial prediction of soil properties using environmental correlation. - Geoderma $89: 67-94$

MDSMA (1995): Memoria esplicativa, mapa forestal. - Sectretaría Nacional de Recursos Naturales, La Paz, Bolivia

Merrill, S.D., A.L. Black, D.W. Fryrear, A. Saleh, T.M. Zobeck, A.D. Halvorson \& D.L. TANAKA (1999): Soil wind erosion hazard of spring wheat-fallow as affected by longterm climate and tillage. - Soil Science Society of America Journal 63 : 1768-1777

Mertens, B., D. Kaimowitz, A. Puntodewo, J. Vanclay \& P. Mendez (2004): Modelling deforestation at distinct geographic scales and time periods in Santa Cruz, Bolivia. International Regional Science Review 27 (3) : 271-296

Michels, K., J.P.A. LAMERS \& A. BuERKERT (1998): Effects of windbreak species and mulching on wind erosion and millet yield in the Sahel. - Experimental Agriculture 34 : 449-464

MinASNy, B. \& A.B. MCBRATney (2001): The Australian soil texture boomerang: a comparison of the Asutralien and USDA/FAO soil particle-size classification system. Australian Journal of Soil Research 39 : 1443-1451

Miller, A.J., R. Amundson, I.C. Burke \& C. Yonker (2004): The effect of climate and cultivation on soil organic $\mathrm{C}$ and N. - Biogeochemistry $67: 57-72$

Millington, A.C., X.M. Velez-Liendo \& A.V. Bradley (2003): Scale dependence in multitemporal mapping of forest fragmentation in Bolivia: implications for explaining temporal trends in landscape ecology and applications to biodiversity conservation. ISPRS Journal of Photogrammetry \& Remote Sensing 57 : 289-299

MoHnheIM, F. \& G. KÖSTER (1982): Die wirtschaftliche Erschliessung des Department Santa Cruz (Bolivien) seit Mitte des 20. Jahrhunderts. - Erdkundliches Wissen Heft 56, Geographische Zeitschrift

MonTENEGRo H.R.M. (1987): Estimación de las susceptibilidades a erosion de los suelos y los riesgos de degradación erosiva bajo diferentes sistemas de cultivo en el area de HuaytuYapacani. - Thesis Universidad Autonoma "Gabriel Moreno", Facultad de Sciencias Agricolas, Santa Cruz, Bolivia

Montes DE OCA, I. (1997): Geografia y Recursos Naturales de Bolivia. - $3^{\text {rd }}$ Edition, La Paz, Bolivia

Moore, I.D., P.E. Gessler, G.A. Nielsen \& G.A. Peterson (1993): Soil attribute prediction using terrain analysis. - Soil Science Society of America Journal 57 : 443-452

MoraleS, I. (1993): Monitoreo del Bosque en el Departamento de Santa Cruz, periodo 1988/89 - 1992/93.- Proyecto de Proteccion de los Recursos Naturales en el Departamento de Santa Cruz (Componente Proyecto Tierras Bajas)

Morales, I. (1996): Monitoreo del Bosque en el Departamento de Santa Cruz, periodo 1992/93 - 1994 Proyecto de Proteccion de los Recursos Naturales en el Departamento de Santa Cruz (Componente Proyecto Tierras Bajas)

Morgenroth, S. (1999): Sozioökonomische Rahmenbedingungen und Landnutzung als Bestimmungs-faktoren der Bodenerosion in Entwicklungsländern - Eine überregionale 
empirische Analyse im Kontext der Agrarentwicklung. - PhD-thesis, Humboldt University Berlin, 239 pp.

MÜLLER, D. (2003): Land-use change in the central highlands of Vietnam. A spatial econometric model combining satellite imagery and village survey data. - PhD-thesis, Institute of Rural Development, University of Göttingen

Müller, M.M.L, M.F. Guimarães, T. Desjardins \& D. Mitja (2004): The relationship between pasture degradation and soil properties in the Brazilian Amazon: a case study. Agriculture, Ecosystems and Environment 103 : 279-288

Murty, D., M.U.F. Kirschbaum, R.E. McMurtrie \& H. McGilvray (2002): Does conversion of agricultural land change soil carbon and nitrogen? A review of the literature. - Global Change Biology 8 : 105-123

Myers, N., R.A. Mittermeier, C.G. Mittermeier, G.A.B. DA FonsecA, \& J. Kent (2000): Biodiversity hotspots for conservation priorities. - Nature $403: 853-858$

Navarro, G. \& M. Maldonado (2002): Geografía ecológica de Bolivia. Vegetación y Ambientes Acuáticos. - Fundación Simon I. Patiño, Cochabambam, 719 pp.

NAVARro, G. (1995): Clasificación de la vegetación de la región de Lomerío en el Departamento de Santa Cruz, Bolivia. - Proyecto BOLFOR, Documento Técnico 10

NEEMANN, W. (1991): Bestimmung des Bodenerodierbarkeitsfaktors für winderosionsgefährdete Böden Norddeutschlands. - Geologisches Jahrbuch, F25, 131 pp.

Neill, C., C.C. Cerri, J.M. Melillo, B.J. Feigl, P.A. Steudler, J.F.L. Moraes \& M.C. PICCOLO (1997): Stocks and dynamics of soil carbon following deforestation for pasture in Rondonia. - in: Lal, R., J.M. Kimble, R.F. Follett \& B.A. Stewart [Eds.]: Soil processes and the carbon cycle: 9-28, CRC Press, Boca Raton

Nelson, G.C. \& J. Geoghegan (2002): Deforestation and land use change: sparse data environments. - Agricultural Economics 27 : 201-216

NeMES, A., J.H.M. WÖSTEN, A. Lilly \& J.H. OUdE VoshaAR (1999): Evaluation of different procedures to interpolate particle-size distributions to achieve compatibility within soil databases. - Geoderma $90: 187-202$

NeufElD, H., D.V.S. RescK \& M.A. AyARZA (2002): Texture and land-use effects on soil organic matter in Cerrado Oxisols, Central Brazil. - Geoderma 107 : 151-164

Odeh, I.O.A., A.B. McBratney \& D.J. Chittleborough (1994): Spatial prediction of soil properties from landform attributes derived from a digital elevation model. - Geoderma 63 : 197-214

Odeh, I.O.A., A.B. McBratney \& D.J. Chittleborough (1995): Further results on prediction of soil properties from terrain attributes heterotopic cokriging and regressionkriging. - Geoderma $67: 215-225$

OKE, T.R. (1987): Boundary layer climates. $-2^{\text {nd }}$ ed. Methuen, London, 435 pp.

Oldeman, L.R., R.T.A. HAKKeling \& W.G. Sombroek (1991): World Map of the Status of Human-induced Soil Degradation. Global Assessment of Soil Degradation GLASOD. ISRIC Wageningen, UNEP Nairobi

OrellanA, M., R.G. BARBER, \& O. DíAz (1990): Effects of deep tillage and fertilization on the population, growth and yield of soya during an exceptionally wet season on a compacted sandy loam, Santa Cruz. - Soil \& Tillage Research 17 : 47-61 
PACHECO, P. (1998): Estilos de desarollo, deforstación y degradación de los bosques en las Tierras Bajas de Bolivia, - Centro de Estudios para el Dasarollo Laboral y Agrario (CEDLA), Serie: Bosques y Sociedad No. 2, La Paz, 389 pp.

PACHECO, P. (2001): Deforestation and Forest Degradation in Lowland Bolivia. - in: Wood, C.H. \& R. PORRO [Eds.]: Land use and deforestation in the Amazon, University Press of Florida, Gainsville

PAINTER, M. (1995) Upland-lowland production linkages and land degradation in Bolivia. in: PainTer, M. \& W.H. Durham [Eds.]: The Social Causes of Environmental Destruction in Latin America : 133-168, University of Michigan Press, Ann Arbor

Pan, W.K.Y., S.J. Walsh, R.E. Bilsborrow, B.G. Frizzelle, C.M. Erlien \& F. BaQuero (2004): Farm-level models of spatial patterns of land use and land cover dynamics in the Ecuadorian Amazon. - Agriculture, Ecosystems and Environment 101 : 117-134

PARK, S.J. \& P. VLEK (2002): Environmental correlation of three-dimensional soil spatial variability: a comparision of three adaptive techniques. - Geoderma 109:117-140

Paustian, K., J.Six, E.T. Elliott \& H.W. Hunt (2000) : Management options for reducing $\mathrm{CO}_{2}$ emissions from agricultural soils. - Biogeochemistry $48: 147-163$

Peri, L.P. \& M. Bloomberg (2002): Windbreaks in southern Patagonia, Argentina: A review of research on growth models, windspeed reduction and effects on crops. - Agroforestry Systems $56: 129-144$

PhiRi, S., E. AmÉzQuita, L.M. Rao \& B.R. Singh (2001): Disc harrowing intensity and its impact on soil properties and plant growth of agropastoral systems in the Llanos of Colombia. - Soil \& Tillage Research $62: 131-143$

Pimentel, D. \& N. KounAng (1998): Ecology of soil erosion in ecosystems. - Ecosystems 1 : 416-426

PinARD, M. A., F. E. Putz, D. Rumiz, R. Gusman, \& A. JARDim (1999): Ecological characterization of tree species for guiding forest management decisions in seasonally dry forests in Lomerio, Bolivia. - Forest Ecology and Management 113 : 201-214

Posadas, A.N.D., D. GimÉnez, M. BitTelli, C.M.P. VAZ \& M. Flury (2001): Multifractal characterization of soil particle-size distributions. - Soil Science Society of America Journal $65: 1361-1367$

Post, W.M. \& K.C. KWON (2000): Soil carbon sequestration and land-use change: processes and potential. - Global Change Biology $6: 317-327$

Post, W.M., R.C. Izaurralde, L.K. MAnN \& N. Bliss (2001): Monitoring and verifying changes of organic carbon in soil. - Climatic Change 51 : 73-99

Rafiqpoor, D., Nowicki, C., Villarpando, R., Jarvis, A., Sommer, J. H., Jones, P. \& IBISCH, P. L. (2004): The factor that most influences the distribution of biodiversity: the climate. - In: IBISCH, P. L. and MÉRIDA, G. [Eds.], Biodiversity: the richness of Bolivia. State of knowledge and conservation. Ministerio de Desarrollo Sostenible y Planificacion / Editorial FAN

READ, J.M. \& N.S.-N. LAM (2002): Spatial methods for characterizing land cover and detecting land-cover changes for the tropics. - International Journal of Remote Sensing 23 (12) : 2457-2474

Reich, P., H. Eswaran \& F. BeinRoth (2001): Global dimensions of vulnerability to wind and water erosion. - in: STOTT, D.E., R.H. MOHTAR \& G.C. StEINHARDT [Eds.]: Sustaining 
the global farm : $838-846,10^{\text {th }}$ International Soil Conservation Organization Meeting, Purdue University

REICOSKY, D.C. (1997): Tillage-induced CO2 emission from soil. - Nutrient Cycling in Agroecosystems $49: 273-285$

RESCK, D.V.S. (1998): Agricultural intensification systems and their impotential acidity on soil and water quality in the Cerrados of Brazil. - in: R. LAL [Ed.] Soil quality and agricultural sustainability : 288-300. Ann Arbor Press, Chelsea, MI.

RICHARDS, J.A. \& X. JiA (1999): Remote sensing digital image analysis. - Springer-Verlag, Berlin, $240 \mathrm{pp}$.

RiezeBos, H.T. \& A.C. LOERTS (1998): Influence of land use change and tillage practice on soil organic matter in southern Brazil and eastern Paraguay. - Soil Tillage and Research 49 : 271-275

RonchaIL, J. (1986): Variaciones Climáticas invernales en Amazonía Boliviana. Programa Climatólogico y Hidrológico de la Cuenca Amazónica Boliviana (PHICAB). - Servicio Nacional de Meteología e Hidrología (SENAHMHI), La Paz

RousSEVA, S.S. (1997): Data transformation between soil texture schemes. - European Journal of Soil Science $48: 749-758$

Roth, C.H., B. Meyer, H.-G. Frede \& R. DerPsch (1988): Effect of mulch rates and tillage systems on infiltrability and other soil physical properties of an Oxisol in Paraná, Brazil. Soil Tillage and Research $11: 81-91$

Ruíz, A.D. (1987): Evaluación de la Aptitud de los Suelos del Area Río Yapacani - Huaytu Para. - Thesis Universidad Autonoma "Gabriel Moreno", Facultad de Sciencias Agricolas, Santa Cruz

Ryan, P.J., N.J. McKenzie, D. O’Connell, A.N. LoughHead, P.M. LePPert, D. JacQuier \& L. ASHTON (2000): Integrating forest soils information across scales: spatial prediction of soil properties under Australian forests. - Forest Ecology and Management 138 : 139157

SÁ, J.C. De M., C.C. Cerri, W.A. Dick, R. Lal, S.P. Venske Filho, M.C. Piccolo \& B.E. FeIGL (2001): Organic Matter Dynamics and Carbon Sequestration Rates for a Tillage Chronosequence in a Brazilian Oxisol. - Soil Science Society of America Journal 65 : 1486-1499

SAHANI, U. \& N. BEHERA (2001): Impact of deforestation on soil physicochemical characteristics, microbial biomass and microbial activity of tropical soil. - Land Degradation and Development 12 : 93-105

SAIKH, H., C. VARADACHARI \& K. GHOSH (1998) Changes in carbon, nitrogen and phosphorus levels due to deforestation and cultivation: A case study in Simlipal National Park, India. - Plant and Soil 198 : 137-145

SÁnchez-Azofeia, G.A., R.C. HARriss \& D.L. Skole (2001): Deforestation in Costa Rica: A Quantitative Analysis Using Remote Sensing Imagery. - Biotropica 33 (3) : 378-384

SAXTON, K., D. ChANDLER \& W. SCHILlinger (2001): Wind erosion and air quality research in the northwest U.S. Columbia plateau: Organization and progress. - in: STOTT, D.E., R.H. Mohtar \& G.C. STEINHARDT [eds.]: Sustaining the global farm. Selected papers from the $10^{\text {th }}$ International Soil Conservation Organization Meeting : 766-770

Schachtschabel, P., H.-P. Blume, G. BrÜmmer, K.H. Hartge \& U. SchwertmanN (1998): Scheffer / Schachtschabel - Lehrbuch der Bodenkunde. - 14. Auflage, Stuttgart 
SCHIMEL D.S. (1995): Terrestrial ecosystem and carbon cycle. - Global Change Biology 1 : 7 91

SCHLESINGER, W.H. \& J.A. ANDREWS (2000): Soil respiration and the global carbon cycle. Biogeochemistry $48: 7-20$

Schlichting, E., H.-P. Blume \& K. Stahr (1995): Bodenkundliches Praktikum. - Pareys Studientexte 81, 2. Aufl., Blackwell Wissenschafts-Verlag Berlin, Wien

Schloeder, C.A., N.E. Zimmerman \& M.J. Jacobs (2001): Comparison of methods for interpolating soil properties using limited data. - Soil Science Society of America Journal $65: 470-479$

SchUlTZ, J. (2000): Handbuch der Ökozonen. - Stuttgart : Ulmer

Servant, M., J. Maley, B. TurcQ, M.L. Absy, P. Brenac, M. Fourbier, \& M.P. Ledru (1993): Tropical forest changes during the late Quaternary in African and South American lowlands. - Global and Planetary Change 7 : 25-40

SHANG, C. \& H. THIESSEN (2000): Carbon turnover and carbon-13 natural abundance in organo-mineral fractions of a tropical dry forest soil under cultivation. - Soil Science Society of America Journal 64 : 2149-2155

ShIRAZI, M., L. BOERSMA \& C.B. Johnson (2001): Particle-size distributions: Comparing texture systems, adding rock and predicting soil properties. - Soil Science Society of America Journal $65: 300-310$

SiVAKUMAR, M.V.K \& C. VAlEnTIN (1997): Agroecological zones and the assessment of crop production potential. - Philosophical Transactions of The Royal Society B 352 : 907916

Six, J., E.T. ElliotT \& K. Paustian (1999): Aggregate and Soil Organic Matter Dynamics under Conventional and No-Tillage Systems. - Soil Science Society of America Journal 63 : $1350-1358$

SiX, J., C. Feller, K. Denef, S.M. Ogle, J.C.de Moraes Sa \& A. Albrecht (2002) : Soil organic matter, biota and aggregation in temperate and tropical soils - Effects of no-tillage. - Agronomie 22 : 755-775

Skaggs, T.H., L.M. Arya, P.J. Shouse \& B.P. Mohanty (2001): Estimating particle-size distribution from limited soil texture data. - Soil Science Society of America Journal 65 : 1038-1044

Skidmore, A.K., C. Varekamp, L. Wilson, E. Knowles \& J. Delaney (1997): Remote sensing of soils in a eucalypt forest environment. - International Journal of Remote Sensing $18(1): 39-56$

Soll Survey Staff (2003): Keys to Soil Taxonomy. - United States Department of Agriculture (USDA ) - Ninth Edition

SOlOMON, D., J. LEHMANN \& W. ZECH (2000): Land use effects on soil organic matter properties of chromic luvisols in semi-arid northern Tanzania: carbon, nitrogen, lignin and carbohydrates. - Agriculture, Ecosystems and Environment 78 : 203-213

Sommer, M., M. Wehrhan, M. Zipprich, U. Weller, W. ZU Castell, S. Ehrich, B. TANDLER \& T. SElige (2003): Hierarchial data fusion for mapping soil units at field scale. - Geoderma 112 : 179-196

SONNEVELD, B.G.J.S. (2003): Formalizing expert judgements in land degradation assessment: A case study for Ethiopia. - Land Degradadation and Development 14 : 347-361 
Soria Martínez, C. A. (1996): Esperanzas y Realidades. Colonización en Santa Cruz. Cuadernos de Investigación, $\mathrm{N}^{\circ} 49$, Centro de investigación y promoción del campesino (Cipca), La Paz.

STEININGER, M.K. (2000): Satellite estimation of tropical secondary forest above-ground biomass: data from Brazil and Bolivia. - International Journal of Remote Sensing $21(6,7)$ : $1139-1157$

Steininger, M.K., C.J. Tucker, J.R.G. Townshend, T.J. Killeen, A. Desch, V. Bell \& P. ERSTS (2001a): Tropical deforestation in the Bolivian Amazon. - Environmental Conservation $28(2): 127-134$

Steininger, M.K., C.J. Tucker, P. Ersts, T.J. Killeen, Z. Villegas \& S.B. Hecht (2001b): Clearance and Fragmentation of Tropical Deciduous Forest in the Tierras Bajas, Santa Cruz, Bolivia. - Conservation Biology 15 (4) : 856-866

STEININGER, M.K. (1996): Tropical secondary forest regrowth in the Amazon: area, age and change estimation with Thematic Mapper data. - International Journal of Remote Sensing $17: 9-27$

STERK, G. (2003): Causes, consequences and control of wind erosion in sahelian Africa: A review. -Land Degradation and Development 14 : 95-108

Stoorvogel, J.J. \& E.M.A. Smaling (1998): Research on soil fertility decline in tropical environments: integration of spatial scales. - Nutrient Cycling in Agroecosystems 50 : 151158

Stout, J.E. \& T.M. ZoBecK (1996): The Wolfforth field experiment: A wind erosion study. Soil Science 161(9) : 616-632

SYERS, J.K. (1997): Managing soils for long-term productivity. - Philosophical Transactions of The Royal Society B 352:1011-1021

TaboadA, M.A., O.A. BARbosa, M.B. Rodríguez \& D.J. Cosentino (2004): Mechanisms of aggregation in a silty loam under different simulated management regimes. - Geoderma $123: 233-244$

TAN, Z. \& R. LAL (2005) : Carbon sequestration potential estimates with changes in land use and tillage practice in Ohio, USA. - Agriculture, Ecosystems and Environment 111 : 140152

Tarboton, D.G. (2003): Terrain Analysis Using Digital Elevation Models in Hydrology. $23^{\text {rd }}$ ESRI International Users Conference 2003, San Diego, California

Tenberg, A., M. DA Veiga, S.C.F. Dechen \& M.A. Stocking (1998): Modelling the impact of erosion on soil productivity: A comparative evaluation of approaches on data from southern Brazil. - Experimental Agriculture 34 : 55-71

THIELE, G. \& R. BARBER (1998): Linking with agricultural input suppliers for technology transfer: The adaption of vertical tillage in Bolivia. - Journal of Soil and Water Conservation 53 (1) : 51-56

THIELE, G. (1993a): The dynamics of farm development in the Amazon: The Barbecho crisis model. - Agricultural Systems 42 : 179-197

Thiele, G. (1993b): La Crisis del Barbecho: Una re-evaluación. - Informe Técnico No. 1, Centro de Investigación Agricola Tropical (CIAT), Misión Británica en Agricultura Tropical, Santa Cruz, Bolivia 
Thiele, G. (1995): The displacement of settlers in the Amazon: the case of Santa Cruz, Bolivia. - Human Organization 54 : 273-282

Thomas R. J. \& M.A. AyARZA [Eds.] (1999): Sustainable Land management for the Oxisols of the Latin American savannas: dynamics of soil organic matter and indicators of soil quality. - CIAT Publication No. 312, International Center for Tropical Agriculture, Cali

Tiessen, H. I.H. SAlCEDo \& E.V.S.B. SAMPAiO (1992): Nutrient and soil organic matter dynamics under shifting cultivation in semi-arid northeastern Brazil. - Agriculture, Ecosystems and Environment $38: 139-151$

Townsend, A.R., G.P. Asner, C.C. Cleveland, M.E. Lefer \& M.M.C. Bustamante (2002): Unexpected changes in soil phosphorus dynamics along pasture chronosequences in the humid tropics. - Journal of Geophysical Research 107 : D20, 8067, doi:10.1029/2001JD000650

Trumbore, S.E., E.A. Davidson, P.B. De Camargo, D.C. Nepstad \& L.A. Martinelli (1995): Below-ground cycling of carbon in forest and pastures of eastern Amazonia. Global Biogeochemical Cycles $9: 515-528$

TUCKER, C.J. \& J.R.G. TOWNSHEND (2000): Strategies for monitoring tropical deforestation using satellite data. - International Journal of Remote Sensing $21(6,7): 1461-1471$

TUCKER, C.J. \& Townshend, J.R.G. (2000): Strategies for monitoring tropical deforestation using satellite data. - International Journal of Remote Sensing 21 : 1461-1473

URI, N.D. (2000): Global climate change and the effect of conservation practices in US agriculture. - Environmental Geology 40 (1-2) : 41-52

Urioste, M. \& D. Pacheco (2001): Las Tierras Bajas de Bolivia a fines del siglo XX. Tenencia, uso y acceso a la tierra y los bosques. - Fundación Pieb, La Paz

USDA (1999): Soil Quality Test Kit Guide. - Natural Resources Conservation Service, United States Department of Agriculture

VAN Lynden, G.W.J \& L.R. Oldeman (1997): Soil degradation in South and Southeast Asia. - ISRIC (International Soil Reference and Information Centre), Wageningen

VAN LYNDEN, G.W.J (2000): Soil degradation in Central and Eastern Europe: the assessment of the status of human-induced soil degradation. - Report 5, ISRIC (International Soil Reference and Information Centre), Wageningen

VAn Lynden, G.W.J, S. MAnTel \& A. VAn OOstrum (2004): Guiding principles for the quantitative assessment of soil degradation. With a focus on salinization, nutrient decline and soil pollution. - FAO/ISRIC, FAO Land and Water Publications Series, Miscellaneous Paper 36

Vanclay, J., D. Kaimowitz, A. Puntodewo \& P. Mendez (1999): Spatially explicit model of deforestation in Bolivia. - in: LAumonier, Y., B. King, C. LEGG \& K. RenNolls [Eds.]: Data Management and Modelling using Remote Sensing and GIS for Tropical Forest Land Inventory : 371-382

Verburg, P.H. \& A. VeldKAmp (2001): The role of spatially explicit models in land-use change research: a case study for cropping patterns in China. - Agriculture, Ecosystems and Environment $85: 177-190$

Verburg, P.H., W. Soepboer, A. Veldkamp, R. Limpiada, V. Espaldon \& S.S.A. MASTURA (2002): Modeling the spatial dynamics of regional land use: The CLUE-S model. - Environmental Management $30: 391-405$ 
Vitorello, V.A., C.C. Cerri, F.G. Andreux, C. Feller \& R. Victoria (1989): Organic matter and natural carbon-13 distribution in forested and cultivated Oxisols. - Soil Science Society of America Journal 53 : 773-778.

VLEK, P.L.G., R. KÜHNE \& M. DENICH (1997): Nutrient resources for crop production in the tropics. - Philosophical Transactions of The Royal Society B 352 : 975-985

Wachholtz, R. \& A. Herold-Mergl (2003): Amenaza y vulnerabilidad por cambio de cauce e inundación en la Cuenca baja del Río Grande. - Proyecto "Gestión Interinstitucional de Riesgos" - GTZ (Cooperación técnica alemana), La Paz

WACHHOLTZ, R. (2000): Uso y manejo de recursos naturales en colonias Menonitas. Estudio de las colonias 'Riva Palacio' y 'El Dorado' en el contexto del plan de ordenamiento territorial del municipio de Cabezas, Departamento Santa Cruz. - Proyecto MASRENA (Cooperación técnica boliviano-alemana), Santa Cruz

WBGU (1994): Welt im Wandel - Die Gefährdung der Böden. - Wissenschaftlicher Beirat der Bundesregierung Globale Umweltveränderungen, Jahresgutachten, Economica Verlag, Bonn

WBGU (2003): Über Kioto hinaus denken - Klimaschutzstrategien für das 21. Jahrhundert. Wissenschaftlicher Beirat der Bundesregierung Globale Umweltveränderungen, Sondergutachten, Berlin

Weisbach, C., H. Thiessen \& J.J. Jimenez-Osornio (2002): Soil fertility during shifting cultivation in the tropical Karst soils of Yucatan. - Agronomie $22: 253-263$

WERDING, L. (1977): Geomorphologie und rezente Sedimentation im Chaco Boreal, Bolivien. - Giessener Geologische Schriften 12 : 429-446

Werth, D. \& R. Avissar (2002): The local and global effects of Amazon deforestation. Journal of Geophysical Research $107: 55$ 1-8

West, T.O. \& W.M. Post (2002): Soil organic carbon sequestration rates by tillage and crop rotation: a global data analysis. - Soil Science Society of America Journal 66 : 1930-1946

Whicker, J.J., D.D. BresheArs, P.T. WASIOLEK, T.B. KirchneR, R.A. TAVANI, D.A.SCHOEP \& J.C. RoDGERS (2002): Temporal and spatial variation of episodic wind erosion in unburned and burned semiarid shrubland. - Journal of Environmental Quality 31 : 599-612

WiLKInson, K.M. \& C.R. ElEVITCH (2000): Multipurpose windbreaks: Design and species for Pacific Islands. - in: Elevitch, C.R \& K.M. WiLKInsON [Eds.]: Agroforestry guides for Pacific Islands. Permanent Agriculture Resources, Holualoa, 240 pp.

Wilson, J.P. \& J.C. GALlant (2000): Secondary topographic attributes. - in: WiLson, J.P., Gallant, J.C. [Eds.]: Terrain analysis: principles and applications : 87-131, Wiley and Sons, New York

Woods, C.H. \& D. Skole (1998): Linking satellite, census, and survey data to study deforestation in the Brazilian Amazon. - in: LiVERMAN, D., E.F. MORAN, R.R. RindFusS, P.C. STERN [Eds.]: People and Pixels: Linking Remote Sensing and Social Science : 70-93, National Academy Press, Washington DC.

ZHANG, H., K.H. HARTGE \& H. RINGE (1997): Effectiveness of organic matter incorporation in reducing soil compactability. - Soil Science Society of America Journal 61 : 239-245

Zhang, H., A. Henderson-Sellers \& K. MCGuffie (2001): The compounding effects of tropical deforestation and greenhouse warming on climate. - Climate Change 49:309-338 
ZhaO, W.Z., H.L. XiAO, Z.M. LIU \& J. Li (2005): Soil degradation and restoration as affected by land use change in the semiarid Bashang area, northern China. - Catena 59:173-186

Zhao, M., A.J. Pitman \& T. Chase (2001): The impact of land cover change on the atmospheric circulation. - Climate Dynamics $17: 467-477$

Zobeck, T.M., G. Sterk, R. Funk, J.L. Rajot, J.E. Stout \& R.S. VAn Pelt (2003): Measurement and data analysis methods for field-scale wind erosion studies and model validation.- Earth Surface Processes and Landforms 28 : 1163-1188

Zurayk, R., F.A.C. Sayegh, S. Hamadeh \& A.G. Chehab (2001): A GIS-based methodology for soil degradation evaluation. - in: STOTT, D.E., R.H. MoHTAR \& G.C. STEINHARDT [Eds.]: Sustaining the Global Farm. Selected papers from the $10^{\text {th }}$ International Soil Conservation Organization Meeting : 1082-1089 


\section{KARTENVERZEICHNIS}

BOLFOR (1996): Mapa administrativo forestal del departamento de Santa Cruz; Escala 1 : 1.500.000. Laboratorio de SIG y Teledeteccion

Comite Central Menonita (1995): Colonias Menonitas. - 1 : 500.000, Segunda Edición

Cordecruz \& KFW (1995): Departamento de Santa Cruz, Plan de Uso de Suelo, 1 : 1.500.000. - Proyecto plan de protección de recursos naturales de Santa Cruz, Consorcio IP/CES/KWC

CORDECRUZ \& KFW (1995): Mapa IGM 09: Capacidad de los suelos para uso agropecuario, 1 : 500.000. - Proyecto plan de protección de recursos naturales de Santa Cruz, Consorcio IP/CES/KWC

CordecruZ \& KFW (1995): Mapa IGM 09: Plan de uso del suelo, 1 : 500.000. - Proyecto plan de protección de recursos naturales de Santa Cruz, Consorcio IP/CES/KWC

CORDECRUZ \& KFW (1995): Mapa IGM 09: Potencial forestal, 1 : 500.000. - Proyecto plan de protección de recursos naturales de Santa Cruz, Consorcio IP/CES/KWC

CORDECRUZ \& KFW (1995): Mapa IGM 13: Capacidad de los suelos para uso agropecuario, 1 : 500.000. - Proyecto plan de protección de recursos naturales de Santa Cruz, Consorcio $\mathrm{IP} / \mathrm{CES} / \mathrm{KWC}$

CORDECRUZ \& KFW (1995): Mapa IGM 13: Fisiografia y suelos, 1 : 500.000. - Proyecto plan de protección de recursos naturales de Santa Cruz, Consorcio IP/CES/KWC

CORDECRUZ \& KFW (1995): Mapa IGM 13: Plan de uso del suelo, 1 : 500.000. - Proyecto plan de protección de recursos naturales de Santa Cruz, Consorcio IP/CES/KWC

CORDECRUZ \& KFW (1995): Mapa IGM 13: Potencial forestal, 1 : 500.000. - Proyecto plan de protección de recursos naturales de Santa Cruz, Consorcio IP/CES/KWC

CORDECRUZ \& KFW (1995): Mapa IGM 13: Vegetación, 1 : 500.000. - Proyecto plan de protección de recursos naturales de Santa Cruz, Consorcio IP/CES/KWC

CORDECRUZ \& KFW (1995): Mapa IGM 17: Fisiografia y suelos, 1 : 500.000. - Proyecto plan de protección de recursos naturales de Santa Cruz, Consorcio IP/CES/KWC

EnNS, D. (1996): , ohne Titel' (Karte der Mennonitenkolonien im Department Santa Cruz) 1 : 1.000.000. - Nueva Esperanza

Instituto Geografíco Militar (1972): Vallegrande, 1 : 250.000. - SE 20-11, Serie H 531 (I.G.M.)

Instituto Geografíco Militar (1974): Santa Cruz de la Sierra, 1 : 250.000. - SE 20-6, Serie H 531 (I.G.M.)

Instituto Geografíco Militar (1979): Banados del Izozog, Escala 1 : 250.000. - SE 20-10, Serie H 531 (I.G.M.)

Instituto Geografíco MilitaR (1985): Concepción, 1 : 250.000. - SE 20-3, Serie H 531 (I.G.M.)

Instituto Geografíco Militar (1992): Cotoca, 1 : 250.000. - SE 20-7, Serie H 531, Segunda Impresión (I.G.M.)

Instituto Geografíco Militar (1998): Mapa Fisico de Bolivia, 1 : 1.000.000. - Tercera Edición (I.G.M.) 
MASRENA, IP \& GTZ (1999): Cobertura vegetal y uso de suelo del "Municipio Cabezas". Proyecto MASRENA, IP - GTZ, Museo de Historía Natural Noell Kempff Mercato, Santa Cruz

MDSMA (1995): Mapa forestal de Bolivia. - Ministerio de Desarollo Sotenible y Medio Ambiente, Secretaría Nacional de Recursos Naturales y Medio Ambiente, La Paz, Bolivia

VRIES, A. DE (1995): Uso actual de la tierra departamento de Santa Cruz; Escala 1 : 1.000 .000 . 


\section{ANHANG}

Tab. A1: Richtwerte chemischer Bodeneigenschaften nach CIAT

\begin{tabular}{lccccccc}
\hline & & sehr niedrig & niedrig & mäßig & angemessen & hoch & sehr hoch \\
\hline SOM & {$[\%]$} & $<1,0$ & $1,1-2,4$ & $2,5-4,5$ & & $4,6-9,0$ & $>10$ \\
$\mathrm{~N}$ total [\%] & {$[\%]$} & $<0,05$ & $0,05-0,10$ & $0,10-0,15$ & - & $0,15-0,25$ & $>0,25$ \\
P-Olsen & {$\left[\mathrm{mg} \mathrm{kg}^{-1}\right]$} & $<3,0$ & $3,0-7,0$ & - & $7-15$ & $15-25$ & $>25,0$ \\
P-Troug & {$\left[\mathrm{mg} \mathrm{kg}^{-1}\right]$} & $<5,0$ & $5-25$ & $25-50$ & - & $50-100$ & $>100$ \\
KAK & {$\left[\mathrm{cmol} \mathrm{kg}^{-1}\right]$} & $<6,0$ & $6,0-12,0$ & $12,0-25,0$ & - & $25,0-40,0$ & $>40$ \\
Basensättigung & {$[\%]$} & $<20,0$ & $21-40$ & $41-60$ & - & $61-80$ & $>81$ \\
$\mathrm{Ca}$ & {$\left[\mathrm{cmol} \mathrm{kg}^{-1}\right]$} & $<2,0$ & $2,0-5,0$ & $5,0-10,0$ & - & $10,0-20,0$ & $>20,0$ \\
$\mathrm{Mg}$ & {$\left[\mathrm{cmol} \mathrm{kg}^{-1}\right]$} & $<0,5$ & $0,5-1,5$ & $1,5-4,0$ & - & $4,0-8,0$ & $>8,0$ \\
$\mathrm{~K}$ & {$\left[\mathrm{cmol} \mathrm{kg}^{-1}\right]$} & $<0,1$ & $0,1-0,2$ & $0,2-0,4$ & $0,4-0,7$ & $0,7-1,2$ & $>1,2$ \\
$\mathrm{Na}$ & {$\left[\mathrm{cmol} \mathrm{kg}^{-1}\right]$} & $<0,1$ & $0,1-0,3$ & $0,3-0,7$ & - & $0,7-2,0$ & $>2,0$ \\
\hline
\end{tabular}

Quelle: GUAMAN (1988a, 1988b, 1999), IPORRE (1996)

Tab. A2: Technische Daten der verwendeten Landsat Satellitenbilder

\begin{tabular}{lcccccc}
\hline satellite & \multicolumn{2}{c}{ Landsat 5 TM } & \multicolumn{2}{c}{ Landsat 4 TM } & \multicolumn{2}{c}{ Landsat 7 ETM+ } \\
\hline year & \multicolumn{2}{c}{1984} & \multicolumn{2}{c}{1992} & \multicolumn{2}{c}{2001} \\
path/row & $230 / 72$ & $230 / 73$ & $230 / 72$ & $230 / 73$ & $230 / 72$ & $230 / 73$ \\
date & 30.09 .1984 & 30.09 .1984 & 10.07 .1992 & 10.07 .1992 & 04.08 .2001 & 04.08 .2001 \\
source & USGS & USGS & TRFIC & TRFIC & TRFIC & TRFIC \\
file format & GeoTIFF & GeoTIFF & BSQ & BSQ & HDF & HDF \\
sun azimuth & 71 & 69 & 49 & 48 & 45,73 & 45,03 \\
sun elevation & 53 & 53 & 32 & 31 & 42,11 & 40,94 \\
reference ellipsoid & WGS 84 & WGS 84 & WGS 84 & WGS 84 & WGS 84 & WGS 84 \\
map projection & UTM & UTM & UTM & UTM & UTM & UTM \\
zone & -20 & -20 & -20 & -20 & -20 & Nearest \\
resampling method & Nearest & Nearest & Nearest & Nearest & Nearest & nest \\
& neighbor & neighbor & neighbor & neighbor & neighbor & neighbor \\
Spatial resolution & $30 \mathrm{~m}$ & $30 \mathrm{~m}$ & $28,5 \mathrm{~m}$ & $28,5 \mathrm{~m}$ & $30 \mathrm{~m}$ & $30 \mathrm{~m}$ \\
Cloud Cover & $0-9 \%$ & $0-9 \%$ & $0-9 \%$ & $0-9 \%$ & $0-9 \%$ & $0-9 \%$ \\
Distributor & U.S. Geological Survey & Tropical Rainforest Information Center (TRFIC) \\
\multicolumn{2}{c}{ (USGS) } & \multicolumn{5}{c}{ Michigan State University } \\
\hline
\end{tabular}

Tab. A3: Fehlermatrizen (confusion matrix) der Satellitenbildanalyse (Maximum-Likelihood, ML)

\begin{tabular}{lcccccccc}
\hline \multicolumn{2}{l}{$\begin{array}{l}\text { Overall Accuracy }=\mathbf{( 1 9 8 9} / \mathbf{2 1 7 0}) \\
\text { Kappa Coefficient }=\mathbf{9 . 9 1 5 5}\end{array}$} & $\mathbf{9 1 . 6 5 9 0} \%$ & & & & & & \\
ML 230-72 1984 & Weide & Anbau & Sekundär & Sand & Savanne & Wasser & Wald & Brache \\
\hline Weide & 95,56 & 0,00 & 0,00 & 0,00 & 19,65 & 0,00 & 0,00 & 3,49 \\
Anbau & 0,96 & 98,86 & 0,00 & 0,00 & 0,42 & 0,00 & 1,04 & 0,00 \\
Sekundär & 0,00 & 0,00 & 93,47 & 0,00 & 1,00 & 0,00 & 0,62 & 0,00 \\
Sand & 0,00 & 0,00 & 0,00 & 100,00 & 0,00 & 0,00 & 0,00 & 0,00 \\
Savanne & 3,48 & 0,00 & 2,08 & 0,00 & 77,44 & 0,00 & 0,49 & 1,29 \\
Wasser & 0,00 & 0,00 & 0,00 & 0,00 & 0,00 & 100,00 & 0,00 & 0,00 \\
Wald & 0,00 & 1,14 & 4,44 & 0,00 & 1,50 & 0,00 & 97,86 & 0,00 \\
Brache & 0,00 & 0,00 & 0,00 & 0,00 & 0,00 & 0,00 & 0,00 & 95,22 \\
\hline total & 100,00 & 100,00 & 100,00 & 100,00 & 100,00 & 100,00 & 100,00 & 100,00 \\
\hline
\end{tabular}




\begin{tabular}{|c|c|c|c|c|c|c|c|c|}
\hline \multicolumn{9}{|c|}{$\begin{array}{l}\text { Overall Accuracy }=(1150 / 1209) \\
\text { Kappa Coefficient }=0.9502\end{array}$} \\
\hline ML 230-73 1984 & Weide & Anbau & Sekundär & Sand & Savanne & Wasser & Wald & Brache \\
\hline Weide & 95,52 & 0,51 & 0,00 & 0,00 & 0,00 & 0,00 & 0,00 & 0,00 \\
\hline Anbau & 3,76 & 97,39 & 0,00 & 0,00 & 3,82 & 0,00 & 0,00 & 0,00 \\
\hline Sekundär & 0,00 & 0,00 & 91,50 & 0,00 & 0,00 & 0,00 & 1,19 & 0,00 \\
\hline Sand & 0,00 & 0,00 & 0,00 & 100,00 & 0,00 & 0,00 & 0,00 & 0,00 \\
\hline Savanne & 0,71 & 2,10 & 0,00 & 0,00 & 95,14 & 0,00 & 0,00 & 1,39 \\
\hline Wasser & 0,00 & 0,00 & 0,00 & 0,00 & 0,00 & 100,00 & 0,00 & 0,00 \\
\hline Wald & 0,00 & 0,00 & 8,50 & 0,00 & 0,00 & 0,00 & 98,81 & 0,00 \\
\hline Brache & 0,00 & 0,00 & 0,00 & 0,00 & 1,04 & 0,00 & 0,00 & 98,61 \\
\hline total & 100,00 & 100,00 & 100,00 & 100,00 & 100,00 & 100,00 & 100,00 & 100,00 \\
\hline \multicolumn{9}{|c|}{ Overall Accuracy $=(2269 / 2380) 95.3361 \%$} \\
\hline \multicolumn{9}{|c|}{ Kappa Coefficient $=0.9526$} \\
\hline ML 230-72 1992 & Weide & Anbau & Sekundär & Sand & Savanne & Wasser & Wald & Brache \\
\hline Weide & 96,40 & 1,31 & 0,00 & 0,00 & 14,34 & 0,00 & 0,40 & 2,22 \\
\hline Anbau & 0,11 & 98,26 & 0,00 & 1,85 & 0,00 & 0,00 & 0,00 & 3,15 \\
\hline Sekundär & 0,00 & 0,18 & 98,15 & 0,00 & 0,00 & 0,00 & 1,51 & 0,00 \\
\hline Sand & 0,00 & 0,25 & 0,00 & 98,15 & 0,00 & 0,00 & 0,00 & 0,00 \\
\hline Savanne & 3,49 & 0,00 & 0,00 & 0,00 & 83,58 & 0,00 & 0,73 & 4,44 \\
\hline Wasser & 0,00 & 0,00 & 0,00 & 0,00 & 0,00 & 100,00 & 0,00 & 0,00 \\
\hline Wald & 0,00 & 0,00 & 1,85 & 0,00 & 0,83 & 0,00 & 97,36 & 0,00 \\
\hline Brache & 0,00 & 0,00 & 0,00 & 0,00 & 1,25 & 0,00 & 0,00 & 90,18 \\
\hline total & 100,00 & 100,00 & 100,00 & 100,00 & 100,00 & 100,00 & 100,00 & 100,00 \\
\hline
\end{tabular}

\begin{tabular}{lcccccccc}
\hline $\begin{array}{l}\text { Overall Accuracy }=\mathbf{( 1 5 8 2} / \mathbf{1 6 6 7}) \\
\text { Kappa Coefficient } \mathbf{~} \mathbf{0 . 9 4 8 1}\end{array}$ & $\mathbf{9 4 0 1 0 \%}$ & & & & & & \\
ML 230-73 1992 & Weide & Anbau & Sekundär & Sand & Savanne & Wasser & Wald & Brache \\
\hline Weide & 100,00 & 0,60 & 0,00 & 0,00 & 0,00 & 0,00 & 0,00 & 0,00 \\
Anbau & 0,00 & 99,41 & 0,00 & 0,00 & 0,00 & 0,00 & 0,00 & 0,00 \\
Sekundär & 0,00 & 0,00 & 93,83 & 0,00 & 0,00 & 0,00 & 0,86 & 0,00 \\
Sand & 0,00 & 0,00 & 0,00 & 100,00 & 0,00 & 0,00 & 0,00 & 0,00 \\
Savanne & 0,00 & 0,00 & 0,00 & 0,00 & 94,59 & 0,00 & 0,00 & 11,69 \\
Wasser & 0,00 & 0,00 & 0,00 & 0,00 & 0,00 & 100,00 & 0,00 & 0,00 \\
Wald & 0,00 & 0,00 & 6,17 & 0,00 & 0,00 & 0,00 & 99,15 & 0,00 \\
Brache & 0,00 & 0,00 & 0,00 & 0,00 & 5,42 & 0,00 & 0,00 & 88,32 \\
\hline total & 100,00 & 100,00 & 100,00 & 100,00 & 100,00 & 100,00 & 100,00 & 100,00 \\
\hline
\end{tabular}

\begin{tabular}{lcccccccc}
\hline $\begin{array}{l}\text { Overall Accuracy }=\mathbf{( 2 8 9 4 / 3 0 8 7} \\
\text { Kappa Coefficient } \mathbf{~} \mathbf{0 . 9 3 7 0}\end{array}$ & $\mathbf{9 3 . 7 4 8 0} \%$ & & & & & & \\
ML 230-72 2001 & Weide & Anbau & Sekundär & Sand & Savanne & Wasser & Wald & Brache \\
\hline Weide & 99,18 & 0,23 & 0,00 & 0,00 & 0,78 & 0,00 & 0,00 & 0,36 \\
Anbau & 0,35 & 99,16 & 4,69 & 0,00 & 0,00 & 0,00 & 0,00 & 1,87 \\
Sekundär & 0,00 & 0,08 & 91,15 & 0,00 & 0,00 & 0,00 & 0,20 & 0,56 \\
Sand & 0,00 & 0,00 & 0,00 & 100,00 & 0,00 & 0,00 & 0,00 & 0,00 \\
Savanne & 0,37 & 0,00 & 0,83 & 0,00 & 96,72 & 0,00 & 0,00 & 0,60 \\
Wasser & 0,00 & 0,00 & 0,00 & 0,00 & 0,00 & 100,00 & 0,00 & 0,00 \\
Wald & 0,00 & 0,00 & 3,33 & 0,00 & 0,00 & 0,00 & 99,80 & 0,24 \\
Brache & 0,11 & 0,53 & 0,00 & 0,00 & 2,50 & 0,00 & 0,00 & 96,39 \\
\hline total & 100,00 & 100,00 & 100,00 & 100,00 & 100,00 & 100,00 & 100,00 & 100,00 \\
\hline
\end{tabular}

\begin{tabular}{|c|c|c|c|c|c|c|c|c|}
\hline \multicolumn{9}{|c|}{$\begin{array}{l}\text { Overall Accuracy }=(2840 / 2985) \quad 95.1424 \% \\
\text { Kappa Coefficient }=\mathbf{0 . 9 5 0 6}\end{array}$} \\
\hline ML 230-73 2001 & Weide & Anbau & Sekundär & Sand & Savanne & Wasser & Wald & Brache \\
\hline Weide & 94,47 & 0,25 & 0,00 & 0,00 & 1,62 & 0,00 & 0,22 & 3,18 \\
\hline Anbau & 1,00 & 98,34 & 0,00 & 5,18 & 1,92 & 0,00 & 0,27 & 0,00 \\
\hline Sekundär & 0,19 & 0,08 & 87,42 & 0,00 & 0,00 & 0,00 & 2,74 & 0,00 \\
\hline Sand & 0,00 & 1,00 & 0,00 & 94,82 & 0,00 & 0,90 & 0,00 & 0,00 \\
\hline Savanne & 2,06 & 0,13 & 0,00 & 0,00 & 95,96 & 0,00 & 0,00 & 0,00 \\
\hline Wasser & 0,00 & 0,00 & 0,00 & 0,00 & 0,00 & 99,10 & 0,00 & 0,00 \\
\hline Wald & 0,00 & 0,19 & 12,58 & 0,00 & 0,00 & 0,00 & 95,52 & 0,00 \\
\hline Brache & 2,28 & 0,00 & 0,00 & 0,00 & 0,51 & 0,00 & 1,26 & 96,82 \\
\hline total & 100,00 & 100,00 & 100,00 & 100,00 & 100,00 & 100,00 & 100,00 & 100,00 \\
\hline
\end{tabular}


Tab. A4: Anbaukalender der Hauptanbaufrüchte im Department Santa Cruz

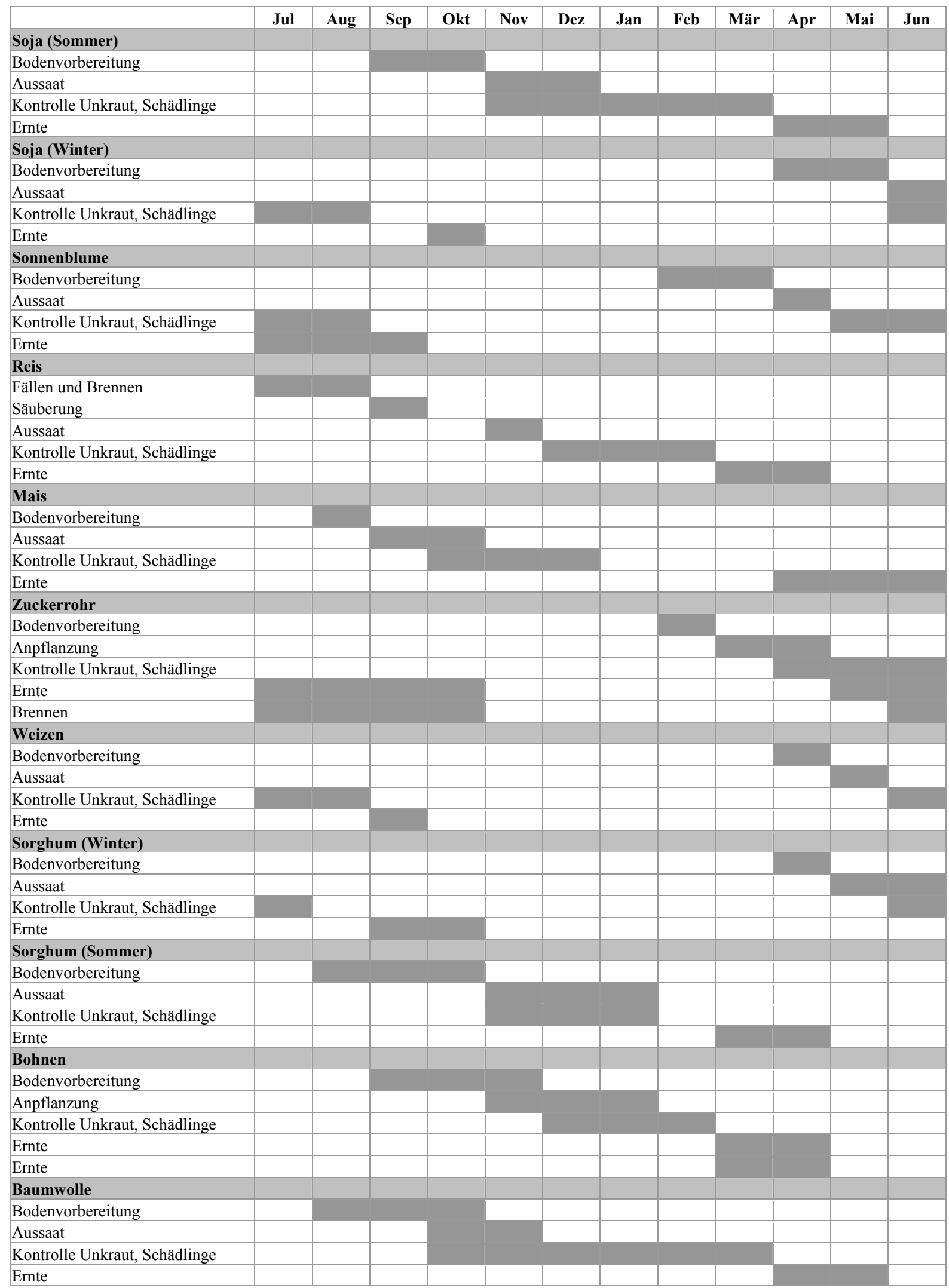

Quelle: CAO (2002) 
Tab. A5: Allgemeine Beschreibung der Standorte und relative Abnahme von Bodenkohlenstoff (SOC) und Bodenstickstoff $\left(\mathrm{N}_{t}\right)$ nach Konversion natürlicher Vegetation in landwirtschaftliche Kulturflächen der durchgesehenen Studien (Literaturreview)

\begin{tabular}{|c|c|c|c|c|c|c|c|c|c|c|c|c|c|c|c|c|c|}
\hline \multirow{4}{*}{ ID } & \multirow{4}{*}{ Autor } & \multirow{4}{*}{ Land } & \multirow{4}{*}{$\begin{array}{l}\text { Tiefe } \\
(\mathrm{cm})\end{array}$} & \multirow{4}{*}{$\begin{array}{l}\text { Nutz- } \\
\text { jahre }\end{array}$} & \multicolumn{8}{|c|}{ Abnahme (\%) } & \multirow{4}{*}{$\begin{array}{l}\text { Boden- } \\
\text { art }\end{array}$} & \multirow{4}{*}{$\begin{array}{l}\text { Boden- } \\
\text { typ }\end{array}$} & \multirow{4}{*}{$\begin{array}{l}\text { Ton } \\
(\%)\end{array}$} & \multirow{4}{*}{$\begin{array}{l}\text { Angabe } \\
\text { Lagerungs- } \\
\text { dichte }\end{array}$} & \multirow{4}{*}{$\begin{array}{l}\text { Anbau- } \\
\text { system }\end{array}$} \\
\hline & & & & & \multirow{2}{*}{\multicolumn{2}{|c|}{ Gehalt }} & \multirow{2}{*}{\multicolumn{2}{|c|}{${ }^{\mathrm{OC}}$ Vorrat }} & \multirow{2}{*}{\multicolumn{4}{|c|}{$N_{t}$}} & & & & & \\
\hline & & & & & & & & & & & & Vorrat & & & & & \\
\hline & & & & & $\Sigma$ & p.a. & $\Sigma$ & p.a. & $\Sigma$ & p.a. & $\Sigma$ & p.a. & & & & & \\
\hline 1 & ABUBAKAR (1997) & Nigeria & 15 & 20 & 40,3 & 2,0 & 32,7 & 1,6 & 53,6 & 2,7 & 47,7 & 2,4 & SL & * & 12 & ja & mechanisiert \\
\hline \multirow[t]{4}{*}{2} & BARBER (1995) & E Bolivien & 15 & 5 & 55,0 & 11,0 & 45,8 & 9,2 & $*$ & $*$ & $*$ & * & SL & Alfisol & 18 & ja & mechanisiert \\
\hline & & & 15 & 14 & 36,0 & 2,6 & 17,8 & 1,3 & $*$ & $*$ & $*$ & $*$ & SL & Alfisol & 22 & $\mathrm{ja}$ & \\
\hline & & & 15 & 18 & 53,0 & 2,9 & 45,3 & 2,5 & $*$ & $*$ & $*$ & $*$ & SL & Inceptisol & 13 & $\mathrm{ja}$ & \\
\hline & & & 15 & 29 & 72,0 & 2,5 & 65,5 & 2,3 & $*$ & $*$ & $*$ & $*$ & SL & Inceptisol & 11 & $\mathrm{ja}$ & \\
\hline \multirow[t]{3}{*}{3} & BARBER et al. (1996) & E Bolivien & 15 & 3 & 42,0 & 14,0 & 38,7 & 12,9 & 44,0 & 14,7 & 41,0 & 13,7 & SL & Alfisol & 20 & & mechanisiert \\
\hline & & & 15 & 5 & 49,0 & 9,8 & 45,8 & 9,2 & 50,0 & 10,0 & 46,4 & 9,3 & SL & Alfisol & 20 & & \\
\hline & & & 15 & 6 & 48,0 & 8,0 & 44,7 & 7,5 & 44,0 & 7,3 & 40,5 & 6,8 & SL & Alfisol & 20 & & \\
\hline \multirow[t]{2}{*}{4} & BELL et al. (1995) & E Australien & 10 & 50 & 72,9 & 1,5 & 64,6 & 1,3 & 60,5 & 1,2 & 48,4 & 1,0 & $\mathrm{C}$ & Oxisol & 65 & & mechanisiert \\
\hline & & & 10 & 70 & 60,5 & 0,9 & 52,7 & 0,8 & 57,1 & 0,8 & 48,7 & 0,7 & $\mathrm{C}$ & Oxisol & 64 & & \\
\hline \multirow[t]{2}{*}{5} & BLASCHKE (1998) & E Bolivien & 20 & 7 & 33,3 & 4,8 & 23,9 & 3,4 & 39,3 & 5,6 & 30,7 & 4,4 & SL & Inceptisol & 12 & ja & traditionell \\
\hline & & & 20 & 24 & 56,7 & 2,4 & 50,1 & 2,1 & 67,9 & 2,8 & 63,0 & 2,6 & LS & Entisol & 8 & ja & \\
\hline \multirow[t]{2}{*}{6} & BONDE et al. (1992) & SE Brasilien & 10 & 12 & 56,0 & 4,7 & 48,8 & 4,1 & $*$ & $*$ & $*$ & $*$ & $\mathrm{C}$ & Oxisol & 55 & & mechanisiert \\
\hline & & & 10 & 50 & 59,0 & 1,2 & 52,1 & 1,0 & $*$ & $*$ & $*$ & * & $\mathrm{C}$ & Oxisol & 55 & & \\
\hline 7 & BRAIMOH \& VLEK (2004) & N Ghana & 20 & 15 & 21,9 & 1,5 & 19,4 & 1,3 & 33,3 & 2,2 & 31,7 & 2,1 & SL & $*$ & 5 & & traditionell \\
\hline 8 & BROWN \& LuGO (1990) & Puerto Rico & 25 & 10 & 55,0 & 5,5 & 61,1 & 6,1 & $*$ & $*$ & $*$ & $*$ & $*$ & Ultisol & $*$ & & mechanisiert \\
\hline \multirow[t]{4}{*}{9} & CENTURION et al. (2004) & Brasilien & 10 & 30 & 57,4 & 1,9 & 32,5 & 1,1 & $*$ & $*$ & $*$ & $*$ & SL & Oxisol & 19 & ja & mechanisiert \\
\hline & & & 10 & 30 & 57,6 & 1,9 & 35,9 & 1,2 & $*$ & $*$ & $*$ & * & $\mathrm{C}$ & Oxisol & 45 & ja & \\
\hline & & & 10 & 30 & 65,2 & 2,2 & 51,1 & 1,7 & $*$ & $*$ & $*$ & $*$ & $\mathrm{C}$ & Oxisol & 42 & ja & \\
\hline & & & 10 & 30 & 61,7 & 2,1 & 55,5 & 1,9 & $*$ & $*$ & $*$ & $*$ & SCL & Oxisol & 22 & ja & \\
\hline \multirow[t]{8}{*}{10} & CIAT (1980) & E Bolivien & 30 & 3 & 14,9 & 5,0 & 12,1 & 4,0 & 16,7 & 5,6 & 14,0 & 4,7 & $\mathrm{C}$ & $*$ & 60 & & traditionell \\
\hline & & & 30 & 3 & 16,7 & 5,6 & 15,0 & 5,0 & 23,1 & 7,7 & 21,5 & 7,2 & SIL & $*$ & 20 & & \\
\hline & & & 30 & 3 & 19,1 & 6,4 & 15,7 & 5,2 & 20,8 & 6,9 & 17,5 & 5,8 & SIC & $*$ & 50 & & \\
\hline & & & 30 & 3 & 38,5 & 12,8 & 35,2 & 11,7 & 35,7 & 11,9 & 32,3 & 10,8 & $\mathrm{CL}$ & $*$ & 32 & & \\
\hline & & & 30 & 4 & 12,8 & 3,2 & 10,3 & 2,6 & 12,5 & 3,1 & 10,1 & 2,5 & SIC & $*$ & 50 & & \\
\hline & & & 30 & 5 & 25,0 & 5,0 & 22,0 & 4,4 & 25,0 & 5,0 & 22,0 & 4,4 & SICL & $*$ & 33 & & \\
\hline & & & 30 & 8 & 57,4 & 7,2 & 51,5 & 6,4 & 58,3 & 7,3 & 52,6 & 6,6 & SIC & $*$ & 50 & & \\
\hline & & & 30 & 15 & 43,5 & 2,9 & 37,8 & 2,5 & 55,0 & 3,7 & 50,5 & 3,4 & SIC & $*$ & 50 & & \\
\hline 11 & CogLe et al. (1995) & NE Australien & 10 & 10 & 56,0 & 5,6 & $*$ & $*$ & $*$ & $*$ & $*$ & $*$ & SL & Alfisol & 10 & & mechanisiert \\
\hline
\end{tabular}




\begin{tabular}{|c|c|c|c|c|c|c|c|c|c|c|c|c|c|c|c|c|c|}
\hline \multirow[t]{6}{*}{12} & DALAL \& MAYER (1986a) & E Australien & 10 & 7 & 34,0 & 4,9 & 31,4 & 4,5 & 28,0 & 4,0 & 25,1 & 3,6 & SL & Alfisol & 18 & ja & mechanisiert \\
\hline & & & 10 & 12 & 19,0 & 1,6 & 17,9 & 1,5 & 25,0 & 2,1 & 24,0 & 2,0 & $\mathrm{C}$ & Vertisol & 59 & ja & \\
\hline & & & 10 & 12 & 34,0 & 2,8 & 31,0 & 2,6 & 32,0 & 2,7 & 28,9 & 2,4 & $\mathrm{CL}$ & Vertisol & 34 & ja & \\
\hline & & & 10 & 18 & 38,0 & 2,1 & 34,4 & 1,9 & 39,0 & 2,2 & 35,4 & 2,0 & $\mathrm{C}$ & Vertisol & 40 & ja & \\
\hline & & & 10 & 18 & 45,0 & 2,5 & 40,1 & 2,2 & 45,0 & 2,5 & 40,1 & 2,2 & $\mathrm{C}$ & Vertisol & 49 & ja & \\
\hline & & & 10 & 26 & 28,0 & 1,1 & 25,1 & 1,0 & 35,0 & 1,3 & 32,4 & 1,2 & $\mathrm{C}$ & Vertisol & 72 & ja & \\
\hline \multirow[t]{6}{*}{13} & DALAL \& MAYER (1986b) & E Australien & 10 & 20 & $*$ & $*$ & 40,2 & 2,0 & $*$ & $*$ & $*$ & $*$ & SL & Alfisol & 18 & & mechanisiert \\
\hline & & & 10 & 23 & $*$ & $*$ & 41,4 & 1,8 & $*$ & * & $*$ & $*$ & $\mathrm{C}$ & Vertisol & 59 & & \\
\hline & & & 10 & 25 & $*$ & $*$ & 39,4 & 1,6 & $*$ & * & * & * & $\mathrm{C}$ & Vertisol & 34 & & \\
\hline & & & 10 & 35 & $*$ & $*$ & 41,9 & 1,2 & $*$ & $*$ & $*$ & $*$ & $\mathrm{C}$ & Vertisol & 40 & & \\
\hline & & & 10 & 45 & $*$ & $*$ & 64,8 & 1,4 & $*$ & * & * & * & $\mathrm{C}$ & Vertisol & 49 & & \\
\hline & & & 10 & 70 & $*$ & $*$ & 39,8 & 0,6 & $*$ & * & * & * & $\mathrm{C}$ & Vertisol & 72 & & \\
\hline 14 & DALAL et al. (2005) & E Australien & 10 & 20 & $*$ & $*$ & 28,0 & 1,4 & $*$ & $*$ & $*$ & $*$ & SL & Alfisol & 12 & & mechanisiert \\
\hline \multirow[t]{2}{*}{15} & D'ANDREA et al. (2004) & Brasilien & 10 & 2 & 10,8 & 5,4 & 4,2 & 2,1 & $*$ & * & $*$ & $*$ & $*$ & Oxisol & 33 & ja & mechanisiert \\
\hline & & & 10 & 17 & 21,6 & 1,3 & 15,3 & 0,9 & 15,7 & 0,9 & 8,7 & 0,5 & $*$ & Oxisol & 33 & ja & \\
\hline \multirow[t]{2}{*}{16} & DoMINIY et al. (2002) & Südafrika & 10 & 30 & 42,3 & 1,4 & 36,9 & 1,2 & $*$ & $*$ & $*$ & $*$ & * & Inceptisol & 18 & & mechanisiert \\
\hline & & & 10 & 30 & 45,8 & 1,5 & 40,6 & 1,4 & $*$ & $*$ & $*$ & $*$ & $\mathrm{C}$ & Oxisol & 62 & & \\
\hline \multirow[t]{2}{*}{17} & GUAMAN (1999) & E Bolivien & 20 & 23 & $*$ & $*$ & $*$ & $*$ & 30,0 & 1,3 & $*$ & $*$ & $\mathrm{C}$ & Vertisol & 53 & & mechanisiert \\
\hline & & & 30 & 23 & $*$ & $*$ & $*$ & * & 56,0 & 2,4 & * & * & SCL & Alfisol & 31 & & \\
\hline 18 & HAJABBASI et al. (1997) & Iran & 30 & 20 & 61,2 & 3,1 & 56,0 & 2,8 & 44,5 & 2,2 & 37,1 & 1,9 & SICL & Inceptisol & 29 & ja & mechanisiert \\
\hline \multirow[t]{2}{*}{19} & HARTEMINK (1998) & Neu Guinea & 15 & 17 & 38,5 & 2,3 & 40,6 & 2,4 & $*$ & $*$ & $*$ & $*$ & $\mathrm{C}$ & Vertisol & 55 & ja & mechanisiert \\
\hline & & & 15 & 17 & 46,6 & 2,7 & 43,8 & 2,6 & $*$ & $*$ & $*$ & $*$ & $\mathrm{CL}$ & Entisol & 30 & ja & \\
\hline \multirow[t]{5}{*}{20} & JAIYEOBA (2003) & N Nigeria & 10 & 3 & 29,2 & 9,7 & 23,6 & 7,9 & $*$ & $*$ & $*$ & $*$ & SL & Alfisol & 15 & $\mathrm{ja}$ & traditionell \\
\hline & & & 10 & 7 & 42,5 & 6,1 & 35,2 & 5,0 & $*$ & $*$ & * & * & SL & Alfisol & 13 & ja & \\
\hline & & & 10 & 11 & 55,8 & 5,1 & 49,5 & 4,5 & 40,0 & 3,6 & 31,5 & 2,9 & SL & Alfisol & 14 & ja & \\
\hline & & & 10 & 15 & 64,6 & 4,3 & 59,9 & 4,0 & 65,0 & 4,3 & 60,3 & 4,0 & SL & Alfisol & 13 & $\mathrm{ja}$ & \\
\hline & & & 10 & 25 & 60,2 & 2,4 & 52,7 & 2,1 & 65,0 & 2,6 & 58,4 & 2,3 & SL & Alfisol & 12 & ja & \\
\hline 21 & JANTALIA et al. (2003) & S Brasilien & 10 & 15 & 39,8 & 2,7 & 33,7 & 2,2 & 41,1 & 2,7 & 33,9 & 2,3 & $\mathrm{C}$ & Oxisol & 63 & & mechanisiert \\
\hline \multirow[t]{2}{*}{22} & KARSTEN (2000) & E Bolivien & 30 & 5 & 55,0 & 11,0 & 51,1 & 10,2 & 57,0 & 11,4 & 53,4 & 10,7 & SL & Alfisol & 17 & ja & traditionell \\
\hline & & & 30 & 7 & 52,0 & 7,4 & 43,3 & 6,2 & 53,0 & 7,6 & 44,5 & 6,4 & SL & Alfisol & 17 & ja & \\
\hline \multirow[t]{5}{*}{23} & KATYAL et al. (2001) & Indien & 20 & 20 & 21,5 & 1,1 & 20,3 & 1,0 & $*$ & $*$ & $*$ & $*$ & $*$ & Alfisol & $*$ & & traditionell \\
\hline & & & 20 & 20 & 40,8 & 2,0 & 39,2 & 2,0 & * & * & * & * & * & Inceptisol & $*$ & & \\
\hline & & & 20 & 20 & 48,6 & 2,4 & 47,8 & 2,4 & $*$ & $*$ & $*$ & $*$ & $*$ & Inceptisol & $*$ & & \\
\hline & & & 20 & 20 & 59,5 & 3,0 & 56,1 & 2,8 & $*$ & * & * & * & * & Mollisol & * & & \\
\hline & & & 20 & 20 & 66,2 & 3,3 & 63,1 & 3,2 & * & $*$ & * & * & * & Mollisol & $*$ & & \\
\hline \multirow[t]{2}{*}{24} & LEITE et al. (2003) & SE Brasilien & 10 & 15 & $*$ & $*$ & 52,3 & 3,5 & $*$ & $*$ & 50,2 & 3,3 & $\mathrm{SC}$ & Ultisol & 40 & & mechanisiert \\
\hline & & & 10 & 15 & $*$ & $*$ & 55,7 & 3,7 & $*$ & $*$ & 52,5 & 3,5 & $\mathrm{SC}$ & Ultisol & 40 & & \\
\hline
\end{tabular}




\begin{tabular}{|c|c|c|c|c|c|c|c|c|c|c|c|c|c|c|c|c|c|}
\hline \multirow[t]{5}{*}{25} & LEMENIH et al. (2005) & Äthiopien & 10 & 7 & 16,8 & 2,4 & 16,6 & 2,4 & 39,2 & 5,6 & 38,7 & 5,5 & SL & Andisol & 16 & & traditionell \\
\hline & & & 10 & 10 & 34,2 & 3,4 & 34,0 & 3,4 & 48,1 & 4,8 & 48,4 & 4,8 & SL & Andisol & 18 & & \\
\hline & & & 10 & 26 & 36,7 & 1,4 & 37,0 & 1,4 & 46,8 & 1,8 & 48,4 & 1,9 & SL & Andisol & 16 & & \\
\hline & & & 10 & 34 & 46,3 & 1,4 & 46,6 & 1,4 & 54,4 & 1,6 & 55,7 & 1,6 & SL & Andisol & 18 & & \\
\hline & & & 10 & 53 & 46,3 & 0,9 & 50,4 & 1,0 & 59,5 & 1,1 & 59,1 & 1,1 & SL & Andisol & 19 & & \\
\hline \multirow[t]{12}{*}{26} & LOBE et al. (2001) & Südafrika & 20 & 10 & 48,5 & 4,9 & 43,9 & 4,4 & 41,3 & 4,1 & 36,0 & 3,6 & SL & Alfisol & 14 & & mechanisiert \\
\hline & & & 20 & 12 & 45,0 & 3,7 & 42,3 & 3,5 & 40,2 & 3,3 & 37,3 & 3,1 & SL & Alfisol & 14 & & \\
\hline & & & 20 & 12 & 51,2 & 4,3 & 49,3 & 4,1 & 42,4 & 3,5 & 40,1 & 3,3 & SL & Alfisol & 14 & & \\
\hline & & & 20 & 20 & 50,3 & 2,5 & 48,4 & 2,4 & 42,4 & 2,1 & 40,2 & 2,0 & SL & Alfisol & 13 & & \\
\hline & & & 20 & 20 & 62,0 & 3,1 & 57,5 & 2,9 & 54,4 & 2,7 & 49,0 & 2,5 & SL & Alfisol & 13 & & \\
\hline & & & 20 & 22 & 50,2 & 2,3 & 47,4 & 2,2 & 48,2 & 2,2 & 45,4 & 2,1 & SL & Alfisol & 13 & & \\
\hline & & & 20 & 30 & 55,0 & 1,8 & 50,3 & 1,7 & 45,0 & 1,5 & 39,4 & 1,3 & SL & Alfisol & 14 & & \\
\hline & & & 20 & 30 & 64,2 & 2,1 & 62,5 & 2,1 & 45,9 & 1,5 & 43,2 & 1,4 & SL & Alfisol & 14 & & \\
\hline & & & 20 & 32 & 54,7 & 1,7 & 52,0 & 1,6 & 45,5 & 1,4 & 42,3 & 1,3 & SL & Alfisol & 14 & & \\
\hline & & & 20 & 40 & 57,6 & 1,4 & 55,7 & 1,4 & 47,1 & 1,2 & 44,7 & 1,1 & SL & Alfisol & 13 & & \\
\hline & & & 20 & 40 & 62,2 & 1,6 & 59,6 & 1,5 & 53,6 & 1,3 & 50,4 & 1,3 & SL & Alfisol & 13 & & \\
\hline & & & 20 & 45 & 58,2 & 1,3 & 53,6 & 1,2 & 50,6 & 1,1 & 45,2 & 1,0 & SL & Alfisol & 13 & & \\
\hline 27 & LUGO \& SANCHEZ (1986) & Puerto Rico & 25 & 60 & $*$ & $*$ & 46,3 & 0,8 & $*$ & $*$ & 28,4 & 0,5 & $*$ & $*$ & $*$ & & mechanisiert \\
\hline \multirow[t]{2}{*}{28} & MACHADO \& SILVA (2001) & S Brasilien & 10 & 11 & $*$ & $*$ & 26,4 & 2,4 & $*$ & $*$ & $*$ & $*$ & $\mathrm{C}$ & Oxisol & 63 & & mechanisiert \\
\hline & & & 10 & 21 & $*$ & $*$ & 40,8 & 1,9 & $*$ & $*$ & $*$ & $*$ & $\mathrm{C}$ & Oxisol & 59 & & \\
\hline \multirow[t]{2}{*}{29} & MAIA \& RIBEIRO (2004) & NE Brasilien & 18 & 2 & 48,6 & 24,3 & 43,9 & 21,9 & $*$ & $*$ & $*$ & $*$ & LS & Ultisol & 10 & ja & mechanisiert \\
\hline & & & 18 & 30 & 42,5 & 1,4 & 37,9 & 1,3 & $*$ & $*$ & $*$ & $*$ & LS & Ultisol & 13 & $\mathrm{ja}$ & \\
\hline \multirow[t]{4}{*}{30} & MARKUSSEN (2000) & E Bolivien & 30 & 5 & 36,0 & 7,2 & 31,5 & 6,3 & 27,8 & 5,6 & 23,1 & 4,6 & SL & Alfisol & 18 & $\mathrm{ja}$ & mechanisiert \\
\hline & & & 30 & 15 & 48,0 & 3,2 & 40,2 & 2,7 & 30,6 & 2,0 & 23,0 & 1,5 & SL & Alfisol & 13 & ja & \\
\hline & & & 30 & 25 & 62,5 & 2,5 & 54,9 & 2,2 & 47,2 & 1,9 & 36,5 & 1,5 & SL & Alfisol & 13 & $\mathrm{ja}$ & \\
\hline & & & 30 & 29 & 61,0 & 2,1 & 54,3 & 1,9 & 50,0 & 1,7 & 41,9 & 1,4 & SL & Alfisol & 16 & $\mathrm{ja}$ & \\
\hline 31 & MCALISTER et al. (1998) & SE Brasilien & 5 & 5 & 55,0 & 11,0 & 50,5 & 10,1 & 50,0 & 10,0 & 45,0 & 9,0 & SL & $*$ & 20 & & traditionell \\
\hline 32 & NEUFELD et al. (1999) & SE Brasilien & 12 & 10 & 27,6 & 2,8 & 25,7 & 2,6 & $*$ & $*$ & $*$ & $*$ & SL & Oxisol & 15 & & mechanisiert \\
\hline \multirow[t]{2}{*}{33} & RESCK $(1998)^{30}$ & NE Brasilien & $*$ & 5 & 49,0 & 9,8 & $*$ & $*$ & $*$ & * & * & * & $\mathrm{SC}$ & Oxisol & 42 & & mechanisiert \\
\hline & & & $*$ & 5 & 69,0 & 13,8 & $*$ & $*$ & $*$ & $*$ & $*$ & $*$ & LS & Entisol & 5 & & \\
\hline \multirow[t]{3}{*}{34} & RIEZEBOS \& LOERTS (1998) & E Paraguay & 10 & 6 & 34,7 & 5,8 & 31,8 & 5,3 & $*$ & $*$ & $*$ & $*$ & $\mathrm{C}$ & Oxisol & 60 & & mechanisiert \\
\hline & & & 10 & 10 & 21,9 & 2,2 & 19,8 & 2,0 & $*$ & $*$ & $*$ & $*$ & $\mathrm{C}$ & Oxisol & 60 & & traditionell \\
\hline & & S Brasilien & 10 & 20 & 23,9 & 1,2 & 21,9 & 1,1 & $*$ & $*$ & $*$ & $*$ & $\mathrm{C}$ & Oxisol & 60 & & mechanisiert \\
\hline \multirow[t]{2}{*}{35} & SAIKH et al. (1998) & Indien & 15 & 5 & 41,0 & 8,2 & 33,6 & 6,7 & 35,2 & 7,0 & 27,1 & 5,4 & $*$ & Alfisol & $*$ & & traditionell \\
\hline & & & 15 & 5 & 57,1 & 11,4 & 45,6 & 9,1 & 48,0 & 9,6 & 34,1 & 6,8 & $*$ & Alfisol & $*$ & & \\
\hline
\end{tabular}

${ }^{30}$ zitiert in: SÁ et al. (2001) 


\begin{tabular}{|c|c|c|c|c|c|c|c|c|c|c|c|c|c|c|c|c|c|}
\hline & & & 15 & 15 & 46,1 & 3,1 & 42,6 & 2,8 & 18,1 & 1,2 & 76,2 & 5,1 & $*$ & Alfisol & $*$ & & \\
\hline & & & 15 & 15 & 64,8 & 4,3 & 58,4 & 3,9 & 53,8 & 3,6 & 3,2 & 0,2 & * & Alfisol & $*$ & & \\
\hline & & & 15 & 15 & 82,5 & 5,5 & 76,0 & 5,1 & 77,7 & 5,2 & 36,7 & 2,4 & * & Alfisol & $*$ & & \\
\hline & & & 15 & 30 & 64,6 & 2,2 & 57,1 & 1,9 & 48,9 & 1,6 & 61,1 & 2,0 & $*$ & Alfisol & $*$ & & \\
\hline & & & 15 & 30 & 70,0 & 2,3 & 58,7 & 2,0 & 67,9 & 2,3 & 29,6 & 1,0 & $*$ & Alfisol & $*$ & & \\
\hline & & & 15 & 60 & 40,5 & 0,7 & 34,4 & 0,6 & $*$ & $*$ & $*$ & * & $*$ & Alfisol & $*$ & & \\
\hline 36 & SHANG \& THIESSEN (2000) & NE Brasilien & 20 & 12 & 27,9 & 2,3 & 25,6 & 2,1 & $*$ & $*$ & $*$ & $*$ & SL & Oxisol & 11 & & traditionell \\
\hline \multirow[t]{2}{*}{37} & SOLOMON et al. (2000) & Tansania & 10 & 3 & 55,6 & 18,5 & 51,4 & 17,1 & 51,0 & 17,0 & 46,2 & 15,4 & SL & Alfisol & 10 & & $*$ \\
\hline & & & 10 & 15 & 56,1 & 3,7 & 51,9 & 3,5 & 51,0 & 3,4 & 46,1 & 3,1 & SL & Alfisol & 10 & & $*$ \\
\hline \multirow[t]{2}{*}{38} & TIESSEN et al. (1992) & NE Brasilien & 18 & 6 & 33,3 & 5,6 & 30,9 & 5,1 & 25,3 & 4,2 & 22,6 & 3,8 & $\mathrm{SL}$ & Oxisol & 11 & & traditionell \\
\hline & & & 18 & 12 & 23,0 & 1,9 & 20,6 & 1,7 & $*$ & $*$ & $*$ & $*$ & SL & Oxisol & 11 & & \\
\hline \multirow[t]{2}{*}{39} & VITORELLO et al.(1989) & SE Brasilien & 20 & 12 & $*$ & $*$ & 48,6 & 4,1 & $*$ & $*$ & $*$ & $*$ & $\mathrm{C}$ & Oxisol & 55 & & mechanisiert \\
\hline & & & 20 & 50 & $*$ & $*$ & 51,4 & 1,0 & $*$ & $*$ & $*$ & $*$ & $\mathrm{C}$ & Oxisol & 55 & & \\
\hline \multirow[t]{3}{*}{40} & ZHAO et al. (2005) & China & 20 & 8 & 54,7 & 6,8 & 52,1 & 6,5 & 37,4 & 4,7 & 33,8 & 4,2 & LS & Mollisol & 9 & ja & traditionell \\
\hline & & & 20 & 30 & 59,9 & 2,0 & 55,3 & 1,8 & 47,2 & 1,6 & 41,1 & 1,4 & LS & Mollisol & 9 & $\mathrm{ja}$ & \\
\hline & & & 20 & 50 & 73,1 & 1,5 & 70,2 & 1,4 & 60,2 & 1,2 & 55,9 & 1,1 & LS & Mollisol & 8 & ja & \\
\hline
\end{tabular}

S: Süd, SE: Südost, NE: Nordost, E: Ost, N: Nord $-\Sigma$ : Gesamtabnahme, p.a.: pro Jahr Anm.: Werte in kursiv sind abgeleitet (s. Kap. 5.1.2.1) 


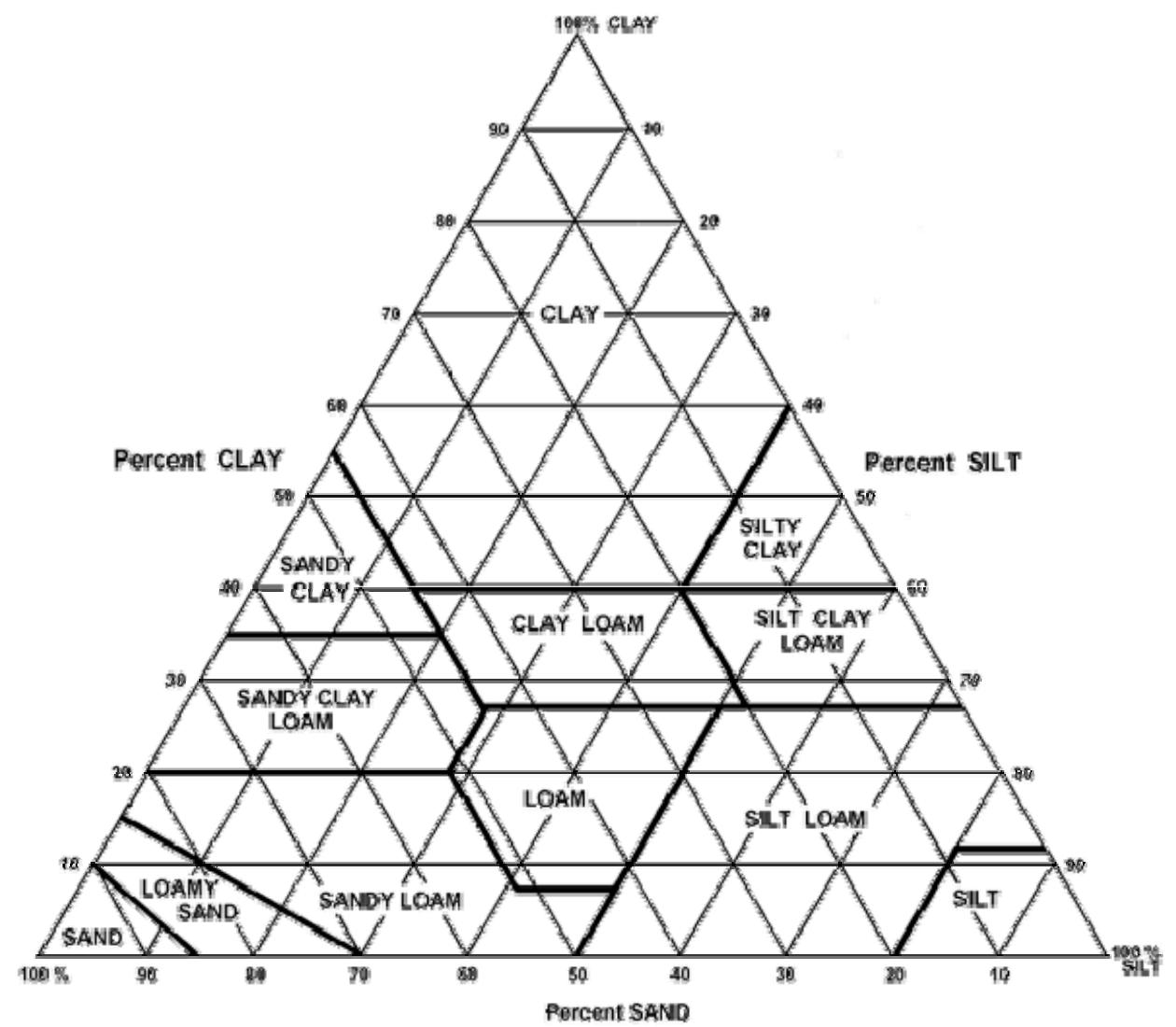

Abb. A1: Korngrößendreieck nach US Soil Taxonomy Quelle: Soil Survey Staff (2003) 


\section{Lebenslauf}

\section{Persönliche Daten}

Name:

Jens-Peter Krüger

Geburtsdatum:

15.11.1966

Geburtsort:

Hamburg

Staatsangehörigkeit:

Deutsch

\section{Schulausbildung}

1973-1977

1977-1986

\section{Zivildienst}

1986-1988

\section{Praktikum}

1988-1989

\section{Studium}

1989-1996

\section{Berufspraxis}

1996-1998

seit 1999

\section{Promotion}

2002-2005
Grundschule Hinsbleek, Hamburg

Gymnasium Harksheider Straße, Hamburg, Abschluss: Abitur

Krankenhaus Großhansdorf, Schleswig-Holstein

landwirtschaftliches Praktikum (Argentinien)

\section{Georg-August Universität Göttingen}

Studium der Geographie mit den

Nebenfächer: Bodenkunde, Geologie, Botanik

Abschluss: Diplom-Geograph

Georg-August Universität Göttingen wissenschaftlicher Mitarbeiter, Geographisches Institut

Abteilung Landschaftsökologie

Georg-August Universität Göttingen wissenschaftlicher Angestellter, Geographisches Institut Abteilung Landschaftsökologie

Georg-August Universität Göttingen

DFG-Projekt: GIS-gestützte Analyse der Waldkonversion und Bodendegradation in Ostbolivien (Santa Cruz de la Sierra) Thema: Waldkonversion und Bodendegradation im tropischen Tiefland von Ostbolivien 